Universidade de São Paulo

Instituto de Física

\title{
Propriedades eletrônicas dos Isolantes Topológicos
}

\author{
Leonardo Batoni Abdalla
}

Orientador: Prof. Dr. Adalberto Fazzio

Tese de doutorado apresentada ao Instituto de Física para a obtenção do título de Doutor em Ciências.

\section{Banca Examinadora:}

Prof. Dr. Adalberto Fazzio (IF-USP)

Prof. Dr. Luis Gregorio Dias da Silva (IF-USP)

Profa. Dra. Lucy Assali (IF-USP)

Prof. Roberto Bechara Muniz (IF-UFF)

Prof. Alex Antonelli (IF-UNICAMP)

São Paulo 


\section{FICHA CATALOGRÁFICA \\ Preparada pelo Serviço de Biblioteca e Informação do Instituto de Física da Universidade de São Paulo}

Abdalla, Leonardo Batoni

Propriedades eletrônicas dos isolantes topológicos.

São Paulo, 2015.

Tese (Doutorado) - Universidade de São Paulo.

Instituto de Física - Depto. de Física dos Materiais e Mecânica.

Orientador: Prof. Dr. Adalberto Fazzio

Área de Concentração: Física

Unitermos: 1. Física; 2.Física da matéria condensada;

3. Física do estado sólido; 4. Física computacional; 5. Topologia. 
"Aprender é a única coisa de que a mente nunca se cansa, nunca tem medo e nunca se arrepende."

Leonardo da Vinci 



\section{Agradecimentos}

A Deus por minha vida, família e amigos.

A Universidade de São Paulo, seu corpo docente, direção e administração pela oportunidade de aprender e trabalhar em um ambiente de alto nível acadêmico.

Ao Prof. Dr. Adalberto Fazzio meu orientador e amigo, pelo suporte no pouco tempo que lhe coube, pelas suas correções e incentivos.

Agradeço a minha mãe a Profa. Dra. Maria Cristina Batoni Abdalla Ribeiro, que me deu apoio incondicional, me incentivou nas horas difíceis, de desânimo e cansaço.

Ao meu pai o Prof. Dr. Elcio Abdalla, por todo o tempo gasto para resolver meus problemas e por manter a certeza, apesar de todas as dificuldades, que independente de quaisquer problemas estaria sempre ao meu lado.

A todas as pessoas que fazem parte da minha família me trazendo sempre alegria e entusiamo. Aos membros do grupo SAMPA e amigos do Instituto de Física pela amizade e apoio. Em especial aos estudantes Carlos Mera Acosta e Alexsandro Kirch pelas calorosas discussões sobre a vida e a física.

Às agências CNPq e o INCT pelo financiamento. 



\begin{abstract}
In the search of a better understanding of the electronic and magnetic properties of topological insulators we are faced with one of its most striking features, the existence of metallic surface states with helical spin texture which are protected from non-magnetic impurities. On the surface these spin channels allows a huge potential for applications in spintronic devices. There is much to do and treating calculations via $A b$ initio simulations allows us a predictive character that corroborates the elucidation of physical phenomena through experimental analysis.

In this work we analyze the electronic properties of topological insulators such as: $(\mathrm{Bi}, \mathrm{Sb})_{2}(\mathrm{Te}, \mathrm{Se})_{3}$, Germanene and functionalized Germanene. Calculations based on DFT show the importance of the separation from interlayers of Van der Waals in materials like $\mathrm{Bi}_{2} \mathrm{Se}_{3}$ and $\mathrm{Bi}_{2} \mathrm{Te}_{3}$. We show that due to stacking faults, small oscillations in the QLs axis (Quintuple Layers) can generate a decoupling of the Dirac cones and create metal states in the bulk phase $\mathrm{Bi}_{2} \mathrm{Te}_{3}$. Regarding the $\mathrm{Bi}_{2} \mathrm{Se}_{3}$ a systematic study of the effects of transition metal impurities was performed. We observed that there is a degeneracy lift of the Dirac cone if there is any magnetization on any axis. If the magnetization remains in plane, we observe a small shift to another reciprocal lattice point. However, if the magnetization is pointing out of the plane a lifting in energy occurs at the very $\Gamma$ point, but in a more intense way. It is important to emphasize that in addition to mapping the sites with their magnetic orientations of lower energy we saw that the lifting in energy is directly related to the local geometry of the impurity. This provides distinct STM images for each possible site, allowing an experimental to locate each situation in the laboratory. We also studied the topological transition in the alloy $\left(\mathrm{Bi}_{x} \mathrm{Sb}_{1-x}\right)_{2} \mathrm{Se}_{3}$, where we identify a trivial and topological insulator for $x=0$ and $x=1$. Despite the obvious existence of such a transition, important details remain unclear. We conclude that doping with non-magnetic impurities provides a good technique for handling and cone engineering this family of materials so that depending on the range of doping we can eliminate conductivity channels coming from the bulk. Finally we studied a Germanene and functionalized Germanene with halogens. Using an asymmetrical functionalization and with the topological invariant $Z_{2}$ we noted that the Ge-I-H system is a topological insulator that could be applied in the development of spinbased devices.
\end{abstract}

Keywords: Topological insulators, Dirac cone, Berry phase, Spin Orbit Coupling, $Z_{2}$ invariant, Chern number, Polarization, functionalized Germanene, Bismuth selenide. 


\section{Resumo}

Na busca de um melhor entendimento das propriedades eletrônicas e magnéticas dos isolantes topológicos nos deparamos com uma das suas caraterísticas mais marcantes, a existência de estados de superfície metálicos com textura helicoidal de spin os quais são protegidos de impurezas não magnéticas. Na superfície estes canais de spin possuem um potencial enorme para aplicações em dispositivos spintrônicos. Muito há para se fazer e o tratamento via cálculos de primeiros princípios por simulações permite um caráter preditivo que corrobora na elucidação de fenômenos físicos via análises experimentais.

Nesse trabalho analisamos as propriedades eletrônicas de isolantes topológicos tais como: $(\mathrm{Bi}, \mathrm{Sb})_{2}(\mathrm{Te}, \mathrm{Se})_{3}$, Germaneno e Germaneno funcionalizado. Cálculos baseados em DFT evidenciam a importância das separações entre as camadas de Van der Waals nos materiais $\mathrm{Bi}_{2} \mathrm{Se}_{3}$ e $\mathrm{Bi}_{2} \mathrm{Te}_{3}$. Mostramos que devido a falhas de empilhamento, pequenas oscilações no eixo de QLs (Quintuple Layers) podem gerar um desacoplamento dos cones de Dirac, além de criar estados metálicos na fase bulk de $\mathrm{Bi}_{2} \mathrm{Te}_{3}$. Em se tratando do $\mathrm{Bi}_{2} \mathrm{Se}_{3}$ um estudo sistemático dos efeitos de impurezas de metais de transição foi realizado. Observamos que há quebra de degenerescência do cone de Dirac se houver magnetização em quaisquer dos eixos. Além disso se a magnetização permanecer no plano, além de uma pequena quebra de degenerescência, há um deslocamento do mesmo para outro ponto da rede recíproca. No entanto, se a magnetização apontar para fora do plano a quebra ocorre no próprio ponto $\Gamma$, porém de maneira mais intensa. Importante enfatizar que além de mapear os sítios com suas orientações magnéticas de menor energia observamos que a quebra da degenerescência está diretamente relacionada com a geometria local da impureza. Isso proporciona imagens de STM distintas para cada sítio possível, permitindo que um experimental localize cada situação no laboratório. Estudamos ainda a transição topológica na liga $\left(\mathrm{Bi}_{x} \mathrm{Sb}_{1-x}\right)_{2} \mathrm{Se}_{3}$, onde identificamos um isolante trivial e topológico para $x=0$ e $x=1$. Apesar de óbvia a existência de tal transição, detalhes importantes ainda não estão esclarecidos. Concluímos que a dopagem com impurezas não magnéticas proporciona uma boa técnica para manipulação e engenharia de cone nesta família de materiais, de forma que dependendo da faixa de dopagem podemos eliminar a condutividade que advém do bulk. Finalmente estudamos superfícies de Germaneno e Germaneno funcionalizado com halogênios. Usando uma funcionalização assimétrica e com a avalição do invariante topológico $Z_{2}$ notamos que o material Ge-I-H é um isolante topológico podendo ser aplicado na elaboração de dispositivos baseados em spin.

Palavras-chave: Isolantes topológicos, Cone de Dirac, Fase de Berry, Interação spin órbita, Invariante $Z_{2}$, Número de Chern, Polarização, Germaneno funcionalizado, Seleneto de Bismuto. 


\section{Sumário}

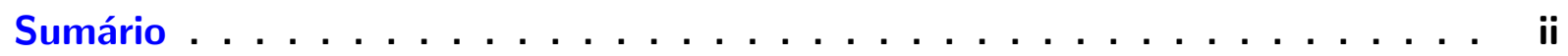

Lista de ilustrações $\ldots \ldots \ldots \ldots \ldots \ldots \ldots \ldots \ldots \ldots$ vi

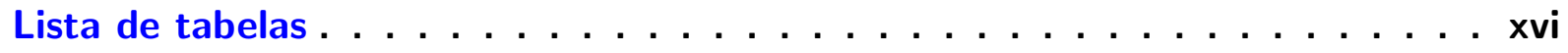

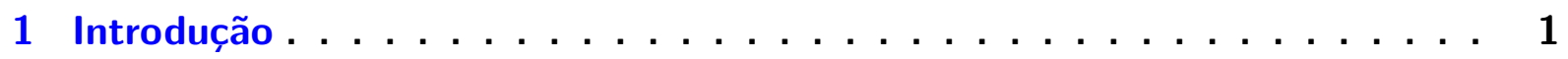

2 Metodologia . . . . . . . . . . . . . . . . 7

2.1 Problema de muitos corpos . . . . . . . . . . . . . . . 7

2.2 Teoria do funcional da densidade $\ldots \ldots \ldots \ldots$

2.2 .1 Descrição do problema . . . . . . . . . . . . . . . . 11

2.3 Teoremas de Hohenberg-Kohn . . . . . . . . . . . . . . . . . . 12

2.4 Equações de Kohn-Sham . . . . . . . . . . . . . . . . . . . . . . . 13

2.4 .1 Escolha da Base . . . . . . . . . . . . . . . . . . . 16

2.4.2 Escolhas para o termo $E_{x c}[\rho] \ldots \ldots \ldots \ldots \ldots$

2.5 Sistemas periódicos e Teorema de Bloch $\ldots \ldots \ldots \ldots \ldots \ldots$

2.5.1 Rede Recíproca e Zona de Brillouin . . . . . . . . . . . . . 18

2.5.2 Condição de contorno cíclica . . . . . . . . . . . . . . . . 20

2.5 .3 Método de ondas planas . . . . . . . . . . . . . . . . . . . . 21

2.5.4 Método de base localizada . . . . . . . . . . . . . . . . . . 22

2.6 Método dos pseudopotenciais . . . . . . . . . . . . . . . . . . 23

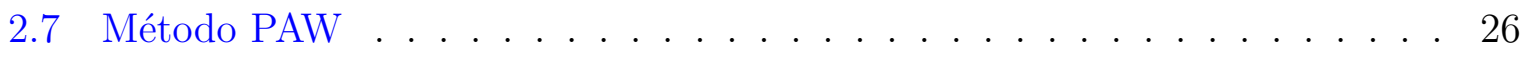

2.8 Mecânica Quântica Relativística . . . . . . . . . . . . . . . . . . . . 29

2.8 .1 Equação de Dirac . . . . . . . . . . . . . . . . . . . . . . . . . . . 29

2.8 .2 Acoplamento Spin Órbita . . . . . . . . . . . . . . . . . 30

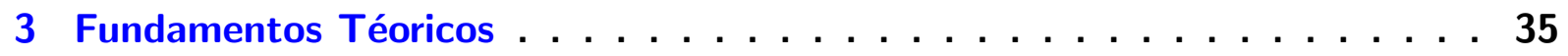


3.1 Topologia de Bandas e suas ferramentas . . . . . . . . . . . . . 35

3.1.1 Operador reversão temporal e Teorema de Kramer . . . . . . . . . . 37

3.2 Invariantes topológicos e fases geométricas em teoria de bandas . . . . . . 39

3.2.1 Fase de Berry . . . . . . . . . . . . . . . . . . . 39

3.2.2 TKNN e número de Chern . . . . . . . . . . . . . . . . . . . 41

3.2.3 Invariante $Z_{2}$ e polarização de reversão temporal . . . . . . . . . . . . 42

3.3 Extensão para 3D . . . . . . . . . . . . . . . . . . . . . . . 45

4 Isolantes Topológicos em 3 e 2 dimensões . . . . . . . . . . . . . . . . . 49

4.0 .1 Propriedades geométricas . . . . . . . . . . . . . . . . . 49

4.0 .2 Propriedades eletrônicas . . . . . . . . . . . . . . . 53

5 Falhas de empilhamento em Isolantes topológicos fortes . . . . . . . . . . 57

5.1 Metodologia . . . . . . . . . . . . . . . . . 58

5.2 Resultados . . . . . . . . . . . . . . . . . . . . 58

$5.2 .1 \quad$ Estabilidade energética . . . . . . . . . . . . . . . . . 58

5.2 .2 Criação de estados metálicos em fase bulk . . . . . . . . . . . . 60

5.2 .3 Corrente de spin em Superfície. . . . . . . . . . . . . . 63

5.3 Conclusão Parcial . . . . . . . . . . . . . . . . . . . . . . . . . . 64

6 Superfície do Isolante Topológico $B i_{2} S e_{3}(\mathbf{1 1 1})$, dopado com metais de transição: Uma análise de primeiros princípios . . . . . . . . . . . . . 65

6.1 Metodologia . . . . . . . . . . . . . . . . . 67

6.2 Resultados . . . . . . . . . . . . . . . . . . . . . . 69

6.2.1 Caso Pristino $\mathrm{Bi}_{2} \mathrm{Se}_{3}(111) \ldots \ldots \ldots$. . . . . . . . . . . . 69

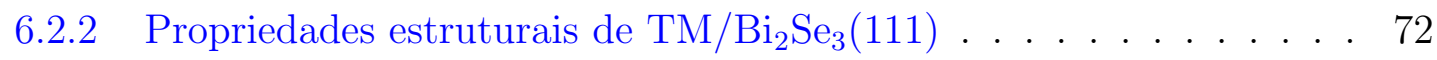

6.2.3 Caracterização topológica de $\mathrm{TM} / \mathrm{Bi}_{2} \mathrm{Se}_{3}(111) \ldots \ldots$. . . . . . . 81

6.3 Conclusões parciais . . . . . . . . . . . . . . . . . . . 88

7 Supressão dos canais de espalhamento em ligas de $\left(B i_{1-x} S b_{x}\right)_{2} S e_{3} \quad \ldots$. . . 91 
7.1 Metodologia . . . . . . . . . . . . . . . . . . 92

7.2 Resultados e discussão . . . . . . . . . . . . . . . . . . . . . . 94

7.3 Conclusões parciais . . . . . . . . . . . . . . . . . . . . . 99

8 Aumento do gap não trivial em Germaneno funcionalizado com Halogênios 101

8.1 Metodologia . . . . . . . . . . . . . . . . . 103

8.2 Resultados . . . . . . . . . . . . . . . . . . . 103

8.3 Conclusões parciais . . . . . . . . . . . . . . . . . . . 109

A Teoria Scanning Tunneling Microscope - STM . . . . . . . . . . 111

A.1 Como funciona um STM . . . . . . . . . . . . . . . . . . 111

A.2 STM Ab Initio . . . . . . . . . . . . . . . . . . . . . . . . . . 112

B Special Quasirandom Structure - SQS . . . . . . . . . . . . . . 115

C Spin- and Angle- Resolved Photoemission Spectroscopy - ARPES and SAR-

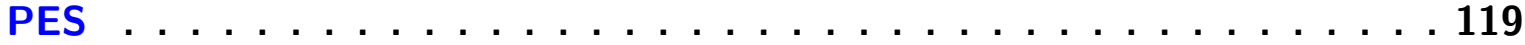

C.1 Princípios básicos . . . . . . . . . . . . . . . . . . . . . . . . . 119

C.1.1 Arpes com spin . . . . . . . . . . . . . . . 120

D Cálculo de $Z_{2}$ no formalismo de ondas planas . . . . . . . . . . . 123

D.1 Metodologia . . . . . . . . . . . . . . . . . . . . . 124

D.2 Materiais 2D com simetria de inversão espacial . . . . . . . . . . . . . . . 124

D.3 Materiais 2D sem simetria de inversão espacial . . . . . . . . . . . . . . 125

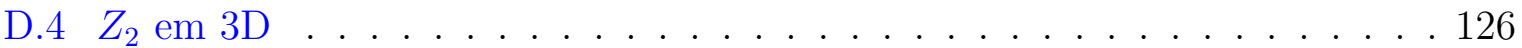

D.5 Implementação do $Z_{2} \ldots \ldots$. . . . . . . . . . . . . . . 127

E Ausência de Retroespalhamento . . . . . . . . . . . . . . . . . 129

F Matemática da matriz $\omega \ldots \ldots$. . . . . . . . . . . . . . . . . . . . . . .

G PROGRAMA AWIREK . . . . . . . . . . . . . . . . 133 
H Método $Z_{2}$ no formalismo de Wannier Charge Centers - WCCs . . . . . . 135

H.1 Formalismo teórico . . . . . . . . . . . . . . . . . . 136

H.2 Procedimento matemático . . . . . . . . . . . . . . . . 138

H.3 Resultado e interpretação . . . . . . . . . . . . . . . . . . . . 139

Referências . . . . . . . . . . . . . . . . . . . . 143 


\section{Lista de ilustrações}

Figura 1 - Em (a) temos a resistência medida em função da voltagem de gate, em poços quânticos de CdTe/HgTe com diferentes larguras d. A curva I foi obtida para uma a mostra com $d=5.5 \mathrm{~nm}$ e as curvas II, III e IV com $d=7.3 \mathrm{~nm}$. As dimensões dos dispositivos correspondentes às curvas I e II são $(20.0 \times 13.3)$ $\mu m^{2}, I I I(1.0 \times 1.0) \mu m^{2}$ e $I V(1.0 \times 0.5) \mu m^{2}$. Todas as medidas foram feitas a $T=30 \mathrm{mK}$ e a campo nulo $(B=0 \mathrm{~T})$. Nota-se que a condutividade para o regime topológico apresenta um plateau de condutividade quantizado em $G=\frac{2 e^{2}}{\hbar}$. (b) Esquema pictórico dos poços [9]. . . . . . . . . . . . .

Figura 2 - Dispersão de banda da superfície de $B i_{0.9} S b_{0.1}$ na direção $\bar{\Gamma}-\bar{M}$. A área sombreada em branco representa a projeção das bandas de bulk baseada em dados de ARPES, os cruzamentos de Fermi dos estados de superfície estão em amarelo, onde próximo de $k_{x} \sim 0.5 \AA^{-1}$ levou-se em conta o fator de dois devido à degenerescência. As linhas vermelhas são guias para os olhos. Nota-se que há 5 cruzamentos entre $\bar{\Gamma}$ e $\bar{M}$ o que indica que estes estados de superfície são topologicamente não triviais [15]. . . . . . . . . . . . . . 4

Figura 3 - (a)-(b) Representam duas superfícies que diferem topologicamente por seu genus, sendo 0 para a esfera e 1 para a rosca. Imagem da referência [62]. 36

Figura 4 - (a) Representação esquemática do espaço real (painel acima) e do espaço recíproco (painel abaixo) de (a) um isolante topológico em $2 D$ e (b) um isolante topológico em 3D. Imagem da referência [63]. . . . . . . . . . . . 36 
Figura 5 - Degenerescência de Kramer, onde ambas as figuras possuem uma dupla degenerescência em $\Gamma_{a} e \Gamma_{b}$. Na figura da esquerda a banda intercepta um número par de vezes o nível de Fermi formando um WTI (Weak Topological Insulator). Na figura da direita a banda intercepta um número impar de vezes o nível de Fermi formando um STI (Strong Topological Insulator). Imagem da referência [62]. . . . . . . . . . . . . . . . . . 39

Figura 6 - Efeito Hall quântico. O campo magnético $\vec{B}$ na direção $\hat{z}$ provoca órbitas ciclotrônicas nos elétrons. A quebra destas órbitas na borda gera uma densidade de corrente elétrica $\vec{j}$ na direção $\hat{x}$ além de um campo elétrico $\vec{E}$ na direção $\hat{y} . \ldots \ldots \ldots \ldots \ldots \ldots \ldots \ldots$

Figura 7 - Estruturas de bandas esquemáticas de duas bordas nos limites dos pontos TRIMs $\Lambda_{a}^{s}$ e $\Lambda_{b}^{s}$ para um isolante trivial(esquerda) e um topológico do tipo $Z_{2}$ (direita). As regiões em azul representam a zona de bulk e para aumentar a visibilidade foi feita um dispersão entre $-\Lambda_{b}^{s} a \Lambda_{b}^{s}$. No isolante topológico temos uma mudança da polarização de reversão temporal, efeito que pode ser visto como uma troca de centros de carga de Wannier, efeito explicado no apêndice H. Imagem da referência [63]. . . . . . . . . . . . . . . . . .

Figura 8 - Representação esquemática da estrutura de banda do material $B i_{1-x} S b_{x}$ em sua fase bulk. Este material muda de um comportamento semi-metal para $x<0.07$, para um comportamento topológico para $0.07<x<0.22$ e de volta para um comportamento semi-metal para $x>0.22$. As bandas de condução $L_{s}$ e valência $L_{a}$ invertem para $x \simeq 0.04$. Imagem da referência [62]. . . . . 4 
Figura 9 - (a) Extensão para 3D dos pontos TRIMs da zona de Brillouin. (b) - (c) Diagramas mostrando as 4 diferentes fases indexadas por $\left(\nu_{0}, \nu_{1} \nu_{2} \nu_{3}\right)$ (b) Sinais de $\delta_{i}$ nos pontos $\Gamma_{i}$ nos vértices do cubo (c) Estrutura de Banda de uma superfície 001 para cada fase. As linhas grossas indicam os possiveis cruzamentos das bandas no nível de Fermi. Os círculos abertos e fechados representam a polarização de reversão temporal para cada TRIM de superfície $\Lambda_{a}$. Imagem da referência [63]. . . . . . . . . . . . . . . . . . . . 48

Figura 10 - (a) Supercélula hexagonal de $\mathrm{Bi}_{2} \mathrm{Se}_{3}$, contendo 15 camadas atômicas mais os vetores primitivos da rede, (b) Recorte no eixo z da rede triangular, onde vemos que há três diferentes tipos de sítios não equivalentes (c) Primeira zona de Brillouin contendo 4 pontos TRIM (Time-Reversal-InvariantMomentum) e a superfície 2D (projeção da zona em 3D) da zona de Brillouin que está representada em linhas azuis. Imagem da referência [84]. . . 50

Figura 11 -Representação estrutural de $\mathrm{Bi}_{2} \mathrm{Se}_{3}$ e $B i_{2} T e_{3}$. (a) Visão Lateral das $3 \mathrm{QL}$ inequivalentes. (b) Visão panorâmica de cada $Q L A, B$ e C, individualmente. As células em verde com suas respectivas setas pretas representam uma orientação fictícia, na idéia de mostar sua equivalência via uma rotação $C_{3}$. $\quad 52$

Figura 12 - Visão panorâmica (a) e lateral (b) da folha de Germaneno, onde se nota uma similaridade com a geometria do grafeno, porém com um "buckling" orientado no eixo z. Em (c) temos a primeira zona de Brillouin desta estrutura hexagonal com os pontos de altas simetria e seus caminhos em vermelho. . . 52

Figura 13 -Representação de dois possiveis recortes de fita do Germaneno funcionalizado com iodo e saturado com hidrogênio nas pontas. Cada recorte esta sinalizado em um quadro azul senda (a) um recorte do tipo Zigzag, e (b) um outro do tipo Armchair. . . . . . . . . . . . . . . . . . . 53

Figura 14 - Visão panorâmica (a) e lateral (b) do Germaneno funcionalizado com iodo. 
Figura 15 -Em (a) temos um diagrama esquemático da evolução dos orbitais atômicos $P_{x, y, z}$ de Bi e Se nas bandas de valência e condução de $\mathrm{Bi}_{2} \mathrm{Se}_{3}$ no ponto $\Gamma$. Os estagios (I), (II), (III) e (IV) representam respectivamente o efeito de se considerar as ligações químicas, simetria de inversão, campo cristalino, e a interação spin órbita. Em azul temos a energia de Fermi. Em (b) e (c) temos as bandas de bulk do sistema $\mathrm{Bi}_{2} \mathrm{Se}_{3}$ com e sem spin órbita. Em ambas as imagens temos em verde e roxo a projeção dos orbitais $p_{z}$ dos átomos de Bi e Se. Nota-se que existe uma inversão de bandas perto do ponto $\Gamma$ quando a interação spin órbita é considerada. . . . . . . . . . . . . 5

Figura 16 - (a) - (b) Dispersões de bandas da superfície do sistema $B i_{2} S_{3}$ para vários tamanhos de $Q L$, sem e com interação spin órbita. Nota-se que sem interação spin órbita não há formação de estados metálicos e além disso, apenas a partir de 4QL os estados metálicos aparecem. . . . . . . . . . . . . . 55

Figura 17 -Em (a) - (b) temos respectivamente bandas projetadas nos orbitais do germanio do sistema Germaneno pristino sem e com spin órbita. Em cada figura temos em verde, azul e vermelho as projeções nos orbitais $s, p_{x}+p_{y}$, e $p_{z}$. Próximo ao ponto $K$ temo um inset mostrando a abertura da degenerescência no ponto $K$ ao se incluir spin órbita, que representa a inversão de paridade da banda..................... . . 55

Figura 18 -Em (a) - (b) temos o mesmo que para a figura 17, para o Germaneno funcionalizado com o Iodo. Aqui a inversão de orbitais do tipo $p_{x y}$ ocorre no ponto $\Gamma$. Logo espera-se um estado metálico na borda neste ponto da zona de Brillouin. . . . . . . . . . . . . . . . . . 56 
Figura 19 -Medidas de ARPES para filmes finos de $B i_{2} S e_{3}$ a temperatura ambiente ao longo da direção $\bar{\Gamma}-\bar{K}$. A curva em rosa em $2 Q L$ representa ajuste do modelo Bernevig-Hughes-Zhang do isolante Hall quântico de spin em duas dimensões. As curvas azuis e vermelhas em 3, 5 e 6 QL representam ajustes para o mesmo modelo com inclusão de Rashba splitting. Imagem da

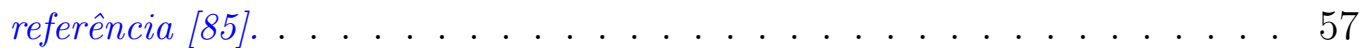

Figura 20 -Modelos estruturais das falhas de empilhamento, à esquerda temos ISF e à direita temos ESF. . . . . . . . . . . . . . . . . . 59

Figura 21 - (a) Representação da separação entre QLs, $\Delta z$. (b) Energia de ligação entre QLs, em função de $\Delta z$, para ISF e ESF em $B i_{2} S e_{3}$ (esquerda) e $B i_{2} \mathrm{Te}_{3}$

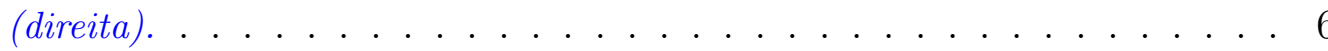

Figura 22 - Estrutura de banda eletrônica de $B i_{2} S e_{3}$ em sua fase bulk com (a) um defeito ISF e (b) um ESF. Regiões em cinza representam projeções do bulk na ausência do defeito. . . . . . . . . . . . . . . 6 61

Figura 23 - O mesmo que a figura 22, para o caso do material $B i_{2} T_{3} . \quad \ldots \ldots$. . . . . 62

Figura 24 - Evolução dos estados em função da distância entre $Q L s, \delta z$ para $B i_{2} \mathrm{Se}_{3}$ : (a) $2.50 \AA$, (b) $3.15 \AA$, (c) $4.15 \AA$, (d) $7.15 \AA$; e para $B i_{2} T e_{3}$ : (e) $2.91 \AA$, (f) $3.26 \AA$, (g) $3.76 \AA$, (h) $7.56 \AA$. Em cinza temos os estados provenientes do bulk pristino de cada sistema. . . . . . . . . . . . . . . . . . . . 62

Figura 25 - Estrutura de banda eletrônica na presença de (a) ESF e (b) ISF em filmes finos de $\mathrm{Bi}_{2} \mathrm{Se}_{3}$. Linhas sólidas representam a estrutura eletrônica de banda da superfície (0001), na presença de SF. Linhas tracejadas representam a estrutura de banda do sistema pristino e regiões em cinza representam os estados de bulk projetados. $E_{D}^{E S F}\left(E_{D}^{I S F}\right)$ indicam a posição em energia do cone de Dirac na presença de ESF (ISF), e $E_{D}^{0}$ indica a posição em energia do cone de Dirac do caso pristino. . . . . . . . . . . . . . . . . . . . . . 64 
Figura 26 - Modelo estrutural do sistema pristino $B i_{2} S_{3}(111)$, em (a) temos as duas primeiras QLs com os dois tipos de átomos presentes, onde em verde e roxo temos Se e Bi, em (b) fizemos um código de cores para identificar as diferentes camadas, aonde temos verde claro, verde escuro, preto, violeta e vermelho, representando respectivamente a primeira, segunda e terceira camada de Se e a primeira e segunda camada de Bi. . . . . . . . . . . . . . 70

Figura 27 - Propriedades eletrônicas do TI Bi $i_{2} S e_{3}(111)$, em (a) temos os STM em um intervalo de energia de $E_{F}= \pm 0.3 \mathrm{eV}$, em (b) linhas sólidas pretas indicam contribuição de todos os átomos para a banda do material, enquanto que em círculos vermelhos temos a contribuição para a banda vinda apenas das primeiras $2 M L$ as quais foram relaxadas. Em (c) temos a $P_{\alpha}(E)$ projetada apenas nas primeiras $2 M L . \ldots \ldots \ldots$. . . . . . . . . . . 71

Figura 28 -Modelos estruturais dos sistemas $T M / B i_{2} S e_{3}(111)$ pós relaxação. Note que este é um caso modelo de Co, porém os outros TM seguem qualitativamente os mesmos deslocamentos, apenas com valores diferentes que podem ser vistos nas tabelas 5 e $6 \ldots \ldots \ldots \ldots$. . . . . . . . . . . . 73

Figura 29 -Modelos estruturais dos casos Bi-sub, Bi-int e Se-int com os respectivos parâmetros que descrevem o sistema cujos valores estão descritos nas ta-

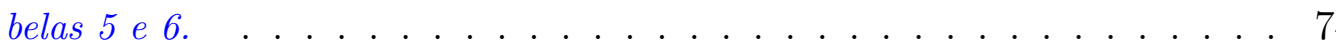

Figura 30 -Detalhes das estruturas adsorvidas pelos metais de transição e seus respectivos parâmetros que descrevem o sistema cujos valores estão descritos nas

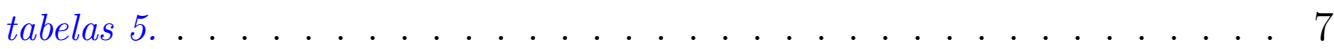

Figura 31 - Imagens simuladas de STM para estados ocupados e vazios dentro de um intervalo de energia de $E_{F}= \pm 0.30 \mathrm{eV} \ldots \ldots . \ldots 78$

Figura 32 -Mesmo que 31, para outras geometrias. . . . . . . . . . . . . . . 80 
Figura 33 -Propriedades eletrônicas dos casos Fe-Bi-sub e Fe-Bi-int. (a)-(b) Bandas de energia com inset da zona de Brillouin e tamanho $\Delta$ do gap. Linhas sólidas pretas nos diagramas de banda indicam a contribuição de todos os átomos para a banda do material, enquanto que círculos vermelhos e quadrados azuis representam respectivamente a contribuição para a banda vinda apenas das primeiras $2 M L$ e do metal de transição, as quais foram relaxadas. Tal contribuição em cada ponto de banda foi selecionada considerando uma porcentagem crítica (maior que 10 \%) da função de partícula única de Kohn-Sham e em (c) e (d) temos a $P_{\alpha}(E)$ projetada, apenas na impureza em linha preta, nas primeiras $2 M L$ em vermelho, e na soma de ambas em

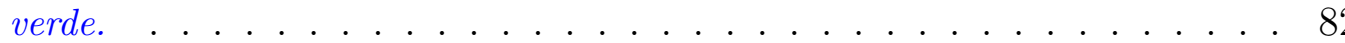

Figura 34 - Quebra de degenerescência dos orbitais atômicos 3d de todos os TM nos ambientes (a) Bi-sub e (b) Bi-int após se ligar respectivamente o campo cristalino e o campo de troca. Em cada caso há duas configurações possíveis, que são High Spin(HS) e Low Spin(LS). . . . . . . . . . . . . . . . . . . . 84

Figura 35 - Mesmo esquema que para a figura 33, para os casos Mn-Bi-sub e Mn-Bi-int. 85

Figura 36 - Mesmo esquema que para a figura 33, para o caso Cr-Bi-sub. . . . . . . . 86

Figura 37 -Mesmo esquema que para a figura 33, para os casos Co-Bi-int e Ni-Bi-int. . 88

Figura 38 - (a) Célula unitária da geometria $\mathrm{Sb}_{2} \mathrm{Se}_{3}$ com seus 3 QLs, com parâmetro de rede hexagonal. Aqui podemos ver três diferentes QLs cada uma girada por $120^{\circ}$ graus em relação à próxima. (b) A fim de evitar falhas de empilhamento decidimos criar todas as configurações possíveis de superfícies. Sabendo que nós temos 3 QLs inequivalentes, só é possível fazer superfícies com terminações do tipo $A-B, A-C$ e $B$-C, como pode ser visto pela escolha de cor diferente para cada QL. (c) Zona de Brillouin da célula de bulk hexagonal com os pontos de alta simetria. (d) Projeção da zona de Brillouin 3D, em sua superfície, com os pontos de alta simetria projetados. . . . . . . 92 
Figura 39 - (a) Estrutura de banda do bulk para a liga $\left(B i_{x} S b_{1-x}\right)_{2} S e_{3}$, entre $0 \%$ e 100 \%. (b) variação do gap de $\left(B i_{x} S b_{1-x}\right)_{2} S e_{3}$. Em verde, temos uma linha reta encaixando todos os tamanhos de gap decrescentes, e em azul, temos outra linha ajustando valores com tamanhos de gap crescente. . . . . . . . . . . . 994

Figura 40 -Estrutura de banda em torno do nivel de Fermi de $\left(B i_{x} S b_{1-x}\right)_{2} S e_{3}$ para (a) $x=0$, (b) $x=0.375$, (c) $x=0.459$, (d) e $x=1$. Os orbitais $p_{z}$ de Bi/Sb são indicados por pontos azuis, enquanto que os orbitais $p_{z}$ do Se são indicados por pontos vermelhos. Ambas as cores possuem um esquema de transparência onde a cor sólida representa 100 \% de contribuição e transparente $0 \% \ldots \ldots \ldots \ldots \ldots \ldots \ldots$

Figura 41 - Estruturas de banda de superfície de $\left(B i_{x} S b_{1-x}\right)_{2} \mathrm{Se}_{3} \mathrm{com}(\mathrm{a}) x=0\left(\mathrm{Sb}_{2} \mathrm{Se}_{3}\right)$, (b) $x=0.33$, (c) $x=0.67$, e (d) $x=1$ (Bi $\left.\mathrm{Se}_{3}\right)$. Em roxo (verde) temos a projeção da banda nas três primeiras (últimas) monocamadas do material. Em (c) - (d) temos as referências em energia da figura 43. Ambas as cores possuem um esquema de transparência onde a cor sólida representa $100 \%$ de contribuição e transparente $0 \%$. . . . . . . . . . . . . . 96

Figura 42 -Bandas das mesmas concentrações que na figura 40, mapeadas na zona de Brillouin inteira. Aqui em vermelho temos a banda de superfície, e em preto a projeção dos estados de bulk. Nota-se a interferência de estados do bulk, em altas concentrações de Bi, podendo proporcionar uma condutividade através o material inteiro. . . . . . . . . . . . . . . . . . . . . 99

Figura 43 - Textura de spin para a parte superior (painel superior) e inferior (painel inferior) do cone de Dirac em (a) $B i_{2} S e_{3}$ e (b) (Bi $\left.i_{0.667} S b_{0.333}\right)_{2} S e_{3}$. A fatia de energia para obter a textura de spin foi de 0.1 eV para a parte desocupada e-0.01 ( 0.0$)$ eV para a parte ocupada de $\mathrm{Bi}_{2} \mathrm{Se}_{3}\left(\left(\mathrm{Bi}_{0.667} \mathrm{Sb}_{0.333}\right)_{2} \mathrm{Se}_{3}\right)$. A escala de cores representa o montante de $S_{z}$. . . . . . . . . . . . . 98 
Figura 44 - (a) Estrutura esquemática do germaneno funcionalizado, Ge-X, onde X representa a funcionalização única por $H$, I ou os compostos binários I-H. $\grave{A}$ esquerda (direita) temos a estrutura de banda para (b) Ge-I e (c) Ge-I-H, sem (com) acoplamento spin órbita. $E_{n g}^{\Gamma}$ e $\Delta$ representam o gap não-trivial que se abriu em $\Gamma$ pelo SOC e o gap indireto no sistema. . . . . . . . . . . 104

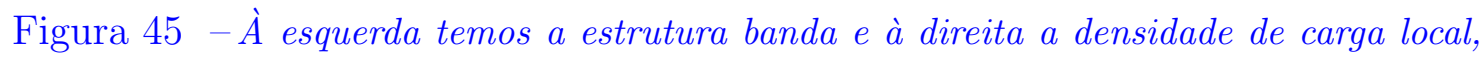
integrada em torno de 0,1 eV do nível de Fermi para nanofitas (a) Armchair e (b) ZigZag de Ge-I e (c) Armchair e (d) ZigZag de Ge-I-H. . . . . . . . 105

Figura 46 - ̀̀ esquerda temos a estrutura de banda para uma nano estrada ZigZag e à direita a densidade de carga locais, integrado em torno de 0,1 eV do nível de Fermi para (a) Ge-I-H e (b) Ge-I. . . . . . . . . . . . . . 106

Figura 47 - (a) Evolução do gap em função da concentração de iodo em Ge-H. (b) Evolução do gap da banda em função da concentração de flúor em Ge-H com diferentes concentrações de iodo. (c) A concentração de iodo como função da concentração de flúor no ponto de transição. (d) Vista esquemática de uma montagem experimental para criar uma nano estrada onde os átomos de hidrogênio são removidos e os átomos de iodo ou de flúor podem ser implantados para formar qualquer padrão desejado, como I e II. . . . . . . 107

Figura 48 - Scanning Tunneling Microscope, em (a) temos o funcionamento de um STM e em (b) visualização da corrente tunelamento entre ponta e amostra. . . . . 112

Figura 49 - Geometria de um experimento de ARPES. A emissão de elétrons pela fonte é especificada pelos ângulos polar $\vartheta$ e azimutal $\varphi$, em seguida os fotoelétrons emitidos da amostra são coletados em um analisador eletrostático. . . . . . 120

Figura 50 -Extensão para $3 D$ dos pontos TRIMs na zona de Brillouin. Imagem da referência $[186] . \ldots \ldots \ldots \ldots \ldots$

Figura 51 -Interface gráfica do Awirek. . . . . . . . . . . . . . . . . 133 
Figura 52 -Evolução dos centros de carga de Wanniers WCCs para materiais 2D. Em vermelho temos a linha de referência. Se durante o meio ciclo entre $\Gamma$ e $M$ houver um número ímpar de cruzamentos entre os WCCs e a linha de referência temos um isolante topológico, caso contrário temos um isolante

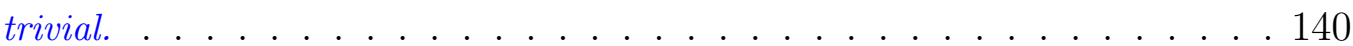

Figura 53 - O mesmo que 52, para materiais 3D. Em vermelho temos a linha de referência. Se durante o meio ciclo entre $\Gamma$ e $M$ houver um número impar de cruzamentos entre os WCCs e a linha de referência para ambos os planos temos um isolante topológico, caso contrário temos um isolante trivial. . . . 141 


\section{Lista de tabelas}

Tabela 1 - Valores dos parâmetros a, c, $\mu$ e $\nu$ para diferentes elementos da família do grupo $D_{3 d^{5}}$. . . . . . . . . . . . . . . . . . . . . . . . . 50

Tabela 2 - Distâncias de $\Delta z$ em A, para cada configuração de Stacking Faults. . . . . . 60

Tabela 3 - As seguintes energias de coesão foram obtidas calculando a energia de bulk por átomo de suas fases mais estáveis e subtraindo pela energia do átomo isolado, onde em cada conta inclui-se correções de spin órbita. Para estes cálculos orbitais de Kohn-Sham foram expandidos em uma base de ondas planas com uma energia de até $270 \mathrm{eV}$. . . . . . . . . . . . . . . . . . 68

Tabela 4 - Energias de formação (em eV) de dopagens substitucionais (Bi-sub em condição rica em Bi e Se-sub em condição rica em Se), intersticiais (int) e adsorvidas (ads) no $\mathrm{TI} \mathrm{Bi}_{2} \mathrm{Se}_{3}$.

Tabela 5 - Geometrias de equilíbrio das impurezas intersticiais e adsorvidas, onde distâncias atômicas (d e h) estão em $\AA$ e ângulos de ligações químicas $(\alpha)$ estão em graus. . . . . . . . . . . . . . . . . . . . . . . . . 75

Tabela 6 - Geometrias de equilíbrio das impurezas substitucionais, onde distâncias atômicas (d e h) estão em $\AA$ e ângulos de ligações químicas ( $\alpha$ ) estão em graus. .............................. 76

Tabela 7 - Módulo dos momentos magnéticos (em $\left.\mu_{B}\right)$ e a orientação da polarização

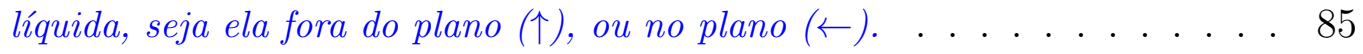




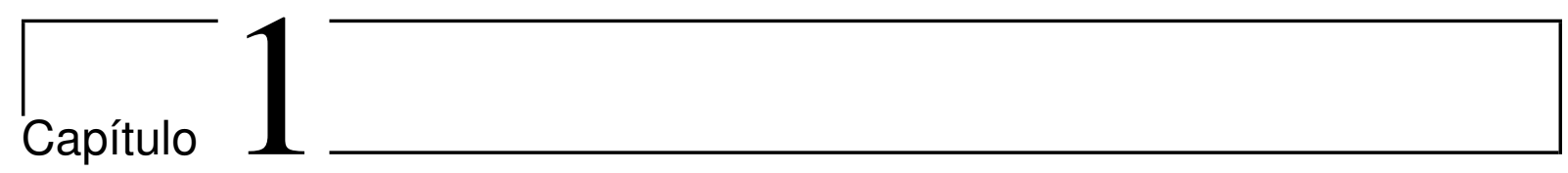

\section{Introdução}

O comportamento de elétrons em diferentes fases da matéria varia drasticamente. $\mathrm{Na}$ antiga compreensão sobre as primeiras fases eletrônicas elaborada por Felix Bloch, acreditava-se que um cristal se comporta como um isolante se todas as bandas de energia permitidas estão separadas por um gap de energia das bandas totalmente vazias. Neste caso nenhum elétron pode se mover em resposta à aplicação de um campo elétrico. Esta definição apresenta falhas quando em 1980 Klaus Von Klitzing, estudando a baixas temperaturas a interface entre dois semicondutores sob a influência de um forte campo magnético, descobre o efeito Hall quântico (QHE - Quantum Hall Effect) [1]. Neste efeito o campo magnético faz com que os elétrons sintam uma força de Lorentz perpendicular, que por sua vez provocam um movimento circular aos elétrons. Este movimento é visto pela mecânica quântica como órbitas com energias quantizadas ou níveis de Landau. Se houver $n$ níveis preenchidos e o resto vazio obtemos o análogo a uma banda energética com gap. Porém na borda deste sistema a quebra das órbitas proporciona uma corrente de carga unidimensional dada por $\sigma_{x y}=\frac{n e^{2}}{\hbar}$, o movimento dos elétrons neste isolante é chamado de quiral no sentido que eles se propagam em um sentido específico na borda. Este material contendo tal efeito é o primeiro isolante topológico (TI - Topological Insulator), na qual "topológico" vem do fato que a função de onda descrevendo os estados eletrônicos geram um espaço de Hilbert com topologia não trivial, ou seja existe uma inversão de bandas energéticas, característica de sistemas com gap, onde para topologia mudar existe a necessidade do gap fechar na interface. Dois anos depois da descoberta do QHE, os pes- 
quisadores Thouless, Kohmoto, Nightingale e Nijs (TKNN) [2], mostraram que a diferença entre o isolante trivial e o isolante Hall quântico é uma questão de topologia. Usando a fórmula de Kubo notaram que o mesmo $n$ da condutividade é um invariante topológico $n \in \mathbb{Z}$ chamado de primeiro número de Chern. Este número pode ser entendido fisicamente em termos da fase de Berry associada às funções de onda de Bloch [3]. Em uma dimensão o invariante pode ser escrito como $n=\int_{-\pi}^{\pi} \frac{d k}{2 \pi} A_{n}(\vec{k})$, onde $A_{n}(\vec{k})$ é a chamada conexão de Berry dada por $A_{n}(\vec{k})=-i\left\langle u_{n \vec{k}}\left|\nabla_{\vec{k}}\right| u_{n \vec{k}}\right\rangle$, com $\left|u_{n \vec{k}}\right\rangle$ funções de Bloch no espaço recíproco. Em 2005 propõe-se um análogo ao QHE denominado efeito spin Hall quântico (QSHE Quantum Spin Hall Effect). Neste efeito idealizado por Kane e Mele [4,5], no sistema grafeno, cuja interação spin órbita abre um gap de energia, dois canais de carga contra propagam-se em cada borda com spin opostos. O resultado da teoria é simplesmente duas cópias do modelo de Haldane [6] onde cada cópia tem um sinal de condutividade Hall opostos para spin up e down. Aqui a interação spin órbita desempenha papel análogo ao campo magnético no QHE, porém mantendo a simetria de reversão temporal (TRS - Time Reversal Symmetry). Este último fato tem consequências profundas na caracterização de um isolante topológico do tipo $Z_{2}$. Devido a esta simetria temos que a função de Bloch é bi-degenerada. Aqui podemos escrever $n=\int_{-\pi}^{\pi} \frac{d k}{2 \pi}\left(A_{n}(\vec{k})^{I}+A_{n}(\vec{k})^{I I}\right)$, sendo $A_{n}(\vec{k})^{I}$ o parceiro Kramer degenerado via reversão temporal de $A_{n}(\vec{k})^{I I}$, e assim temos o novo invariante escrito como $Z_{2}=\int_{-\pi}^{\pi} \frac{d k}{2 \pi}\left(A_{n}(\vec{k})^{I}-A_{n}(\vec{k})^{I I}\right)$. Em um sistema que tenha TRS o número de Chern é zero, mas a consequência física de um invariante não trivial $Z_{2}$ é um bulk isolante e estados de superfície chamados helicoidais de spin, i.e. carga na borda contra-propaga com spin opostos, topologicamente protegidos. A chamada helicidade vem em analogia com a correlação entre spin e momento de uma partícula conhecida como helicity [7]. Hoje sabe-se que uma inversão de bandas ocorrendo em um número impar de pontos invariantes sob simetria de reversão temporal (TRIM - Time Reversal Invariant Momenta) na zona de Brillouin é a chave para se ter o invariante $Z_{2}$ não trivial. Tendo o grafeno um acoplamento spin órbita muito pequeno, criando controvérsias sobre sua abertura de gap não trivial, que corresponde a um gap com o ordenamento de bandas 
invertido ao se considerar o acoplamento spin órbita, pesquisadores partiram para outras propostas.

Em 2006, Bernevig, Hughes e Zhang [8] fizeram a predição teórica que o QSH poderia ser visualizado em poços quânticos de $\mathrm{CdTe} / \mathrm{HgTe} / \mathrm{CdTe}$. Aqui a mudança da largura de HgTe leva a uma inversão de bandas, que por sua vez promove uma transição topológica. A predição foi confirmada por König $[9,10]$ como visto na figura 1 , onde cálculos de condutividade revelaram carga quantizada nas bordas destes poços após certa largura critica do HgTe. Porém este sistema possui um gap não trivial de $\approx 10 \mathrm{meV}$ [11], não

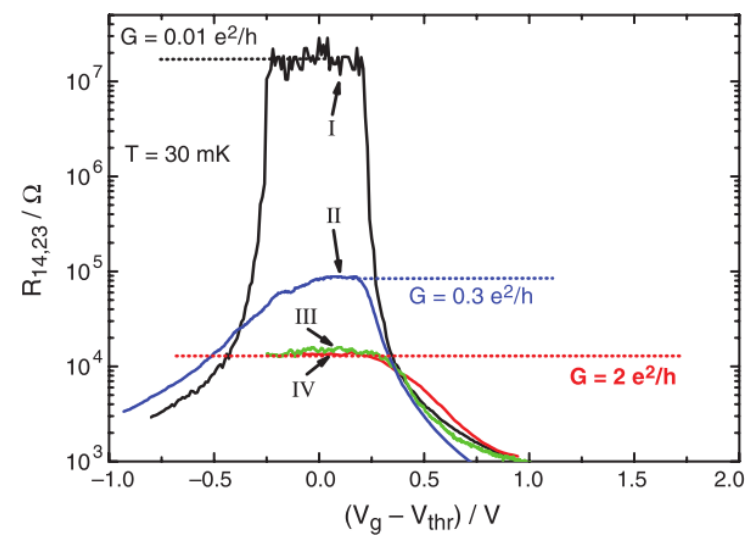

(a) Resistencia em função da voltagem de gate.

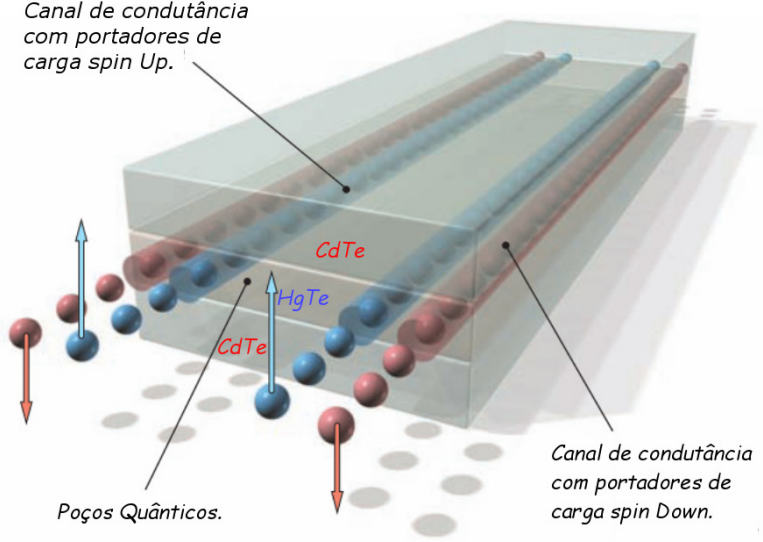

(b) Esquema pictórico dos poços quânticos.

Figura 1: Em (a) temos a resistência medida em função da voltagem de gate, em poços quânticos de $\mathrm{CdTe} / \mathrm{HgTe}$ com diferentes larguras $d$. A curva I foi obtida para uma a mostra com $d=5.5$ $n m$ e as curvas II, III e IV com $d=7.3 \mathrm{~nm}$. As dimensões dos dispositivos correspondentes às curvas I e II são $(20.0 \times 13.3) \mu m^{2}$, III $(1.0 \times 1.0) \mu m^{2}$ e $I V(1.0 \times 0.5) \mu m^{2}$. Todas as medidas foram feitas a $T=30 \mathrm{mK}$ e a campo nulo $(B=0 \mathrm{~T})$. Nota-se que a condutividade para o regime topológico apresenta um plateau de condutividade quantizado em $G=\frac{2 e^{2}}{\hbar}$. (b) Esquema pictórico dos poços [9].

apropriado para spintrônica. Nos anos seguintes houve um importante desenvolvimento teórico possibilitando a descoberta de TIs em três dimensões por Fu, Kane e Mele [12], Moore e Balents [13], ambos em 2007 e Roy [14] em 2009. Foi em 2008 que o estudante de doutorado Hsieh [15] descobre o primeiro TI em 3D via método ARPES (Angle Resolved Photoemission Spectroscopy). Como visto na figura 2. Conclui-se que a liga $\mathrm{Bi}_{0.9} \mathrm{Sb}_{0.1}$ seria um isolante topológico devido a cinco (impar) cruzamentos de banda entre $\bar{\Gamma}$ e $\bar{M}$ que indica uma superfície topologicamente protegida. Esta liga ainda apresenta um gap pequeno $\approx 30 \mathrm{meV}$ [16]. Foi então, em 2009, que uma segunda geração de iso- 


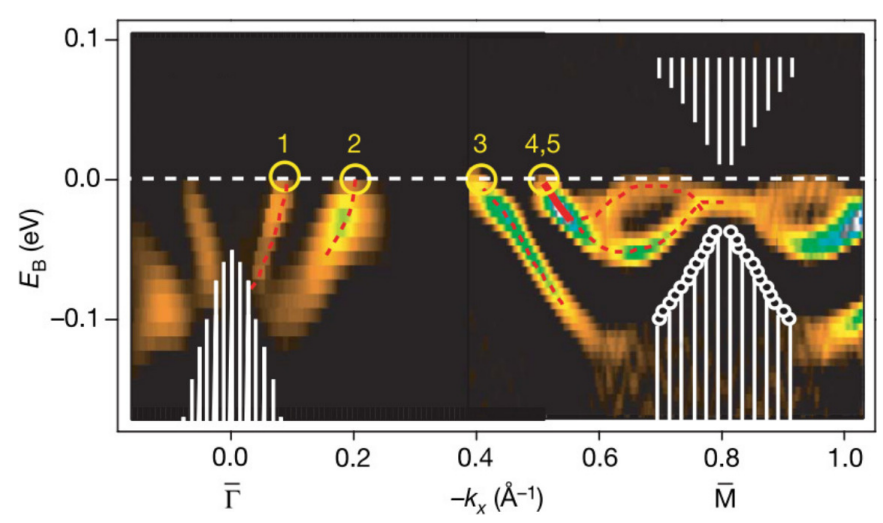

Figura 2: Dispersão de banda da superfície de $B i_{0.9} S b_{0.1}$ na direção $\bar{\Gamma}-\bar{M}$. A área sombreada em branco representa a projeção das bandas de bulk baseada em dados de ARPES, os cruzamentos de Fermi dos estados de superfície estão em amarelo, onde próximo de $k_{x} \sim 0.5 \AA^{-1}$ levou-se em conta o fator de dois devido à degenerescência. As linhas vermelhas são guias para os olhos. Nota-se que há 5 cruzamentos entre $\bar{\Gamma}$ e $\bar{M}$ o que indica que estes estados de superfície são topologicamente não triviais [15].

lantes topológicos estequiométricos da família $(\mathrm{Bi}, \mathrm{Sb})_{2}(\mathrm{Te}, \mathrm{Se})_{3}$ (Chacolgenides ou grupo Tetradymite) foram descobertos teoricamente [17,18] e experimentalmente [18], no qual apenas $\mathrm{Sb}_{2} \mathrm{Se}_{3}$ é topologicamente trivial. Apesar de técnicas como ARPES e STM (Scanning Tunneling Microscope) (ambos descritos nos apêndices C, A) mostrarem um gap significativo de $\mathrm{Bi}_{2} \mathrm{Se}_{3}$ em sua fase bulk por volta de $0.3 \mathrm{eV}$, medidas de transporte detectam uma condutividade importante do bulk [19], o qual esconde os fenômenos exóticos de superfície.

Por conta deste problema alguns grupos tem procurado TIs em $2 \mathrm{D}$ com simetria análoga ao do Grafeno, porém com uma interação spin órbita mais significativa. Em 2011 foi previsto que o Siliceno [20], Germaneno [20], teriam gaps não trivias de $1.55 \mathrm{meV}, 23.9$ meV, respectivamente, porem, apenas o Siliceno [21] foi experimentalmente criado. Além disso, em 2013, encontraram o Staneno [22] com gap não trivial de 0.1 eV. Agora, com a realização que Germaneno funcionalizado com Hidrogênio foi experimentalmente criado [23], (apesar dele não ser um TI), a atenção nesta área voltou-se para funcionalização de halogênios, de forma a aumentar a interação spin órbita. A funcionalização do estanho [22] proporciona gaps não triviais de (0.26-0.34) eV dependendo da dopagem, já o Germaneno [24] possui variações de gap não triviais entre 0.13 e 0.30 eV se aplicado strain, onde a única exceção é Ge-I, um TI sem a necessidade de strain com $0.3 \mathrm{eV}$ de gap não trivial. 
Neste cenário a corrente de spin que existe nos estados de superfície de um TI do tipo $Z_{2}$ podem ser úteis para dispositivos baseados em spin de baixo custo energético. Um atributo importante desta corrente de spin é que ela poderia ser facilmente manipulada mudando o potencial químico através do cone de Dirac com gating. Além disso se eliminarmos um canal de spin no QSH, por exemplo dopando com metais de transição magnéticos, o sistema é levado naturalmente ao que chamamos de QAHE (Quantum Anomalous Hall Effect) [25], com corrente na borda totalmente polarizada - uma excelente alternativa para aplicação de transistores de spin [26].

Nesta tese estamos interessados nas propriedades eletrônicas e magnéticas dos isolantes topológicos $Z_{2}$. Iniciaremos com uma revisão teórica sobre métodos computacionais e aproximações utilizadas nos cálculos de primeiros princípios (Ab Initio) no capítulo 2. Posteriormente, no capítulo 3, introduzimos as ferramentas necessárias para abordar os TI, tanto quanto a teoria por de trás destes sistemas. No capítulo 4 descrevemos a família do $(\mathrm{Bi}, \mathrm{Sb})_{2}(\mathrm{Te}, \mathrm{Se})_{3}$, e o Germaneno pristino e funcionalizado, tanto na parte eletrônica quanto na estrutural. No que concerne os resultados no capítulo 5 estudamos falhas de empilhamento nos $\mathrm{Bi}_{2} \mathrm{Se}_{3}$ e $\mathrm{Bi}_{2} \mathrm{Te}_{3}$, onde vimos que, dependendo do tipo de falha, é possível criar estados metálicos ainda na fase bulk, além de proporcionar um deslocamento em energia do cone de Dirac, removendo da região de bulk, e subsequentemente diminuindo condutividade do bulk. No capítulo 6 fazemos uma investigação sistemática de metais de transição depositados na superfície de $\mathrm{Bi}_{2} \mathrm{Se}_{3}$. Foi feito um mapeamento em energia e em orientações magnéticas de cada impureza em cada sítio possível, além da análise da influência destas propriedades na eletrônica do material. No capítulo 7 estudamos a liga $\left(\mathrm{Bi}_{x} \mathrm{Sb}_{1-x}\right)_{2} \mathrm{Se}_{3}$ para observar a porcentagem crítica na qual ocorre a transição topológica, além de propor uma engenharia de cone na qual nossas superfícies não apresentariam condutividade proveniente do bulk. Finalmente no capítulo 8 analisamos a funcionalização assimétrica do Germaneno, buscando propriedades eletrônicas topológicas, propondo a utilização deste sistema em dispositivos baseados na corrente de spin. 



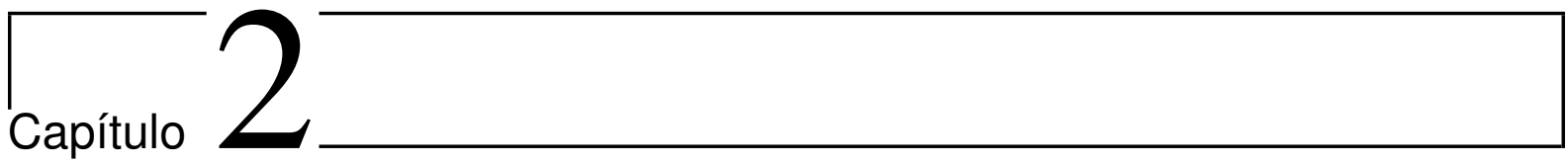

\section{Metodologia}

Neste capítulo abordaremos conceitos necessários para a resolução da equação de onda de muitos corpos, que possibilitará o estudo Ab Initio usando técnicas computacionais. Sendo o objetivo o estudo de propriedades eletrônicas em materiais em nível quântico, além de um bom conhecimento de mecânica quântica, teremos que utilizar alguns métodos e aproximações que devem ser explicados aqui.

\subsection{Problema de muitos corpos}

Qualquer sistema que possua uma estrutura atômica definida, pode ser descrito como um conjunto de núcleos e elétrons que interagem. Para o caso de um sistema cuja escala é nanométrica, efeitos quânticos são importantes. Logo interessa-nos resolver a equação de Schrödinger independente do tempo para um material com M núcleos e N elétrons como vemos na seguinte equação,

$$
\hat{H} \psi(\vec{r}, \vec{R})=E \psi(\vec{r}, \vec{R}),
$$

onde $\hat{H}$ é o operador hamiltoniano total não relativístico e $\psi(\vec{r}, \vec{R})$, é a função de onda total, e as coordenadas $\vec{r}$ e $\vec{R}$, são respectivamente as coordenadas de todos os elétrons e todos os núcleos do sistema. Escrevendo o hamiltoniano no sistema internacional obtemos:

$$
\begin{aligned}
\hat{H}= & -\sum_{i}^{M} \frac{\hbar^{2}}{2 M_{\vec{R}_{i}}} \nabla_{\vec{R}_{i}}^{2}-\frac{\hbar^{2}}{2 m} \sum_{j}^{N} \nabla_{\vec{r}_{j}}^{2}-\frac{1}{4 \pi \epsilon_{0}} \sum_{i}^{M} \sum_{j}^{N} \frac{Z_{\vec{R}_{i}} e^{2}}{\left|\overrightarrow{r_{j}}-\overrightarrow{R_{i}}\right|} \\
& +\frac{1}{4 \pi \epsilon_{0}} \sum_{j}^{N} \sum_{l<j}^{N} \frac{e^{2}}{\left|\overrightarrow{r_{j}}-\overrightarrow{r_{l}}\right|}+\frac{1}{4 \pi \epsilon_{0}} \sum_{i}^{M} \sum_{k<i}^{M} \frac{Z_{\vec{R}_{i}} Z_{\overrightarrow{R_{k}}} e^{2}}{\left|\overrightarrow{R_{i}}-\overrightarrow{R_{k}}\right|}
\end{aligned}
$$




$$
\hat{H}=\hat{T}_{N}+\hat{T}_{e}+\hat{V}_{N e}+\hat{V}_{e e}+\hat{V}_{N N}=\hat{T}_{N}+\hat{H}_{e l e},
$$

sendo $M_{\vec{R}_{i}}, m, Z_{\vec{R}_{i}}$, e e $\epsilon_{0}$ as massas e as cargas do núcleo da posição $\vec{R}_{i}$ e do elétron e a permissividade do vácuo. Ao lado direito da equação (2.3) temos respectivamente os operadores de energia cinética nuclear, energia cinética eletrônica, energia de interação atrativa elétron-núcleo, energia de interação repulsiva elétron-elétron e energia de interação repulsiva núcleo-núcleo, onde os quatro últimos se somam resultando em um hamiltoniano eletrônico. Sem considerarmos o spin, o problema se resume em resolver uma equação com $(M+N)$ corpos interagentes com $3(M+N)$ graus de liberdade, uma tarefa praticamente impossível sem o uso de aproximações, com a exceção de casos simples como o átomo de hidrogênio e a molécula de $H_{2}^{+}$onde um tratamento analítico torna-se possível.

Considerando a desigualdade entre as massas dos elétrons e as dos núcleos, razão que, no melhor dos casos, seria a massa do elétron pela massa do próton igual a $\frac{1}{2000}$, podemos supor que os núcleos se movem bem mais lentamente que os elétrons, de forma que estes últimos seguem instantaneamente o movimento dos núcleos, enquanto permanecem sempre no mesmo estado estacionário do hamiltoniano eletrônico. Esta consideração é chamada de separação de Born-Oppeinheimer [27].

Matematicamente uma das propriedades do hamiltoniano eletrônico é que ele comuta com as diferentes posições do núcleo, ou seja $\left[\hat{H}_{e l e}, \vec{R}\right]=0$. Com ele podemos determinar os autovalores do hamiltoniano eletrônico para particulares posições nucleares, ficando com a seguinte equação:

$$
\hat{H}_{\text {ele }} \chi_{m}(\vec{r} ; \vec{R})=E_{m}(\vec{R}) \chi_{m}(\vec{r} ; \vec{R})
$$

Sabendo que $\chi_{m}(\vec{r} ; \vec{R})$ forma uma base (conjunto completo) ortonormal, podemos agora expandir a própria função de onda total com segue na equação (2.5).

$$
\psi(\vec{r}, \vec{R})=\sum_{m} \phi_{m}(\vec{R}) \chi_{m}(\vec{r} ; \vec{R})
$$

onde $\phi_{m}(\vec{R})$ são as funções de onda descrevendo a evolução do subsistema nuclear em cada um dos autoestados eletrônicos $\chi_{m}(\vec{r} ; \vec{R})$ para cada $\vec{R}$. Ao substituir a equação $(2.5)$ 
na equação de Schrödinger total (2.3), obtemos:

$$
\left[-\sum_{i}^{M} \frac{\hbar^{2}}{2 M_{\overrightarrow{R_{i}}}} \nabla_{\vec{R}_{i}}^{2}+\hat{H}_{\text {ele }}\right] \sum_{m} \phi_{m}(\vec{R}) \chi_{m}(\vec{r} ; \vec{R})=E \sum_{m} \phi_{m}(\vec{R}) \chi_{m}(\vec{r} ; \vec{R}) .
$$

Note que o operador cinético nuclear atua em $\chi_{m}(\vec{r} ; \vec{R})$ que depende das posições dos núcleos. Logo ao multiplicarmos pela esquerda a equação (2.6) por $\chi_{m}^{*}(\vec{r} ; \vec{R})$ e integrar no espaço das coordenadas eletrônicas obtemos a equação (2.7).

$$
-\sum_{i}^{M} \frac{\hbar^{2}}{2 M_{\vec{R}_{i}}} \nabla_{\vec{R}_{i}}^{2} \phi_{n}(\vec{R})+\left(E_{n}(\vec{R})-E\right) \phi_{n}(\vec{R})=\sum_{m} C_{n m}(\vec{R}, \nabla) \phi_{m}(\vec{R}),
$$

onde os termos $C_{n m}(\vec{R}, \nabla)$, chamados de não adiabáticos são dados pela equação (2.8).

$$
\begin{aligned}
C_{n m}(\vec{R}, \nabla) & =\sum_{i}^{M} \frac{\hbar^{2}}{2 M_{\overrightarrow{R_{i}}}}\left(X_{n m} \nabla_{\vec{R}_{i}}+Y_{n m}\right), \\
X_{n m} & =\int \chi_{n}^{*}(\vec{r}, \vec{R}) \nabla_{\vec{R}_{i}} \chi_{m}(\vec{r}, \vec{R}) d^{3} r \\
Y_{n m} & =\frac{1}{2} \int \chi_{n}^{*}(\vec{r}, \vec{R}) \nabla_{\vec{R}_{i}}^{2} \chi_{m}(\vec{r}, \vec{R}) d^{3} r .
\end{aligned}
$$

Na aproximação adiabática consideram-se os termos não diagonais de $C_{n m}$ iguais a zero, e na aproximação de Born Oppenheimer consideram-se todos os termos $C_{n m}$ iguais a zero, nesta última obtemos as equações nucleares (2.9). De qualquer forma estes termos são pequenos por serem proporcionais a razão da massa do elétron e da massa do núcleo.

$$
\left[\hat{T}_{N}+E_{m}(\vec{R})\right] \phi_{m}(\vec{R})=E \phi_{m}(\vec{R})
$$

O movimento nuclear ocorre em um potencial efetivo que é exatamente a energia eletrônica $E_{m}(\vec{R})$. Agora pode-se notar um desacoplamento entre a parte nuclear e eletrônica do sistema. Note que a aproximação de Born Oppenheimer só é válida uma vez que não haja um acoplamento significativo entre diferentes estados eletrônicos $C_{n m} \sim 0$, ou seja desde que não ocorra cruzamento de superfícies de potenciais. Como estamos interessados na parte eletrônica do sistema, vamos trabalhar apenas com a equação (2.4). De qualquer forma, mesmo com esta aproximação, resolver a equação eletrônica é o mesmo que resolver um problema de $N$ corpos interagentes com $3 N$ graus de liberdade. Um ponto importante para ser discutido aqui é a obtenção da configuração nuclear de mínima energia.

$$
i \hbar \frac{\partial \phi_{m}(\vec{R}, t)}{\partial t}=\left(-\sum_{i}^{M} \frac{\hbar^{2}}{2 M_{\overrightarrow{R_{i}}}} \nabla_{\vec{R}_{i}}^{2}+E_{m}(\vec{R})\right) \phi_{m}(\vec{R}, t)
$$




$$
\begin{gathered}
i \hbar \frac{d\langle\vec{R}\rangle}{d t}=\langle[\hat{H}, \vec{R}]\rangle=i \hbar \frac{\langle\vec{P}\rangle}{M} \\
i \hbar \frac{d\langle\vec{P}\rangle}{d t}=\langle[\hat{H}, \vec{P}]\rangle=-i \hbar\left\langle\nabla_{\vec{R}_{i}} E_{m}(\vec{R})\right\rangle \\
M \frac{d^{2}\langle\vec{R}\rangle}{d t^{2}}=-\left\langle\nabla_{\vec{R}_{i}} E_{m}(\vec{R})\right\rangle .
\end{gathered}
$$

Dada a equação de Schrödinger dependente do tempo (2.10), se usarmos o teorema de Ehrenfest da equação (2.11), obtemos a equação de movimento Newtoniana (2.12).

A expressão final de equação de movimento deve-se a Feynman [28] que mostrou, através do teorema chamado de Hellman-Feynman, que se um hamiltoniano depende explicitamente de um parâmetro $\lambda$, como por exemplo, o hamiltoniano eletrônico que depende das coordenadas nucleares, visto na separação Born-Oppenheimer, então seus autovalores e autovetores, dependerão da mesma forma do mesmo parâmetro, como visto na seguinte equação:

$$
\begin{aligned}
\frac{d E_{m}(\lambda)}{d \lambda} & =\left\langle\chi_{m}(\vec{R})\left|\frac{\partial \hat{H}_{e l e}(\lambda)}{\partial \lambda}\right| \chi_{m}(\vec{R})\right\rangle \\
M_{\vec{R}_{i}} \frac{d^{2}\langle\vec{R}\rangle}{d t^{2}} & =-\left\langle\chi_{m}(\vec{R})\left|\frac{\partial \hat{H}_{e l e}(\vec{R})}{\partial \vec{R}}\right| \chi_{m}(\vec{R})\right\rangle
\end{aligned}
$$

Logo se $\lambda=\vec{R}_{i}$, podemos igualar $\nabla_{\vec{R}_{i}} E_{m}(\vec{R})=0$, e achar as posições de equilíbrio do sistema, método chamado de otimização de geometria.

\subsection{Teoria do funcional da densidade}

Devido à necessidade de incluir nas simulações computacionais as correções de spin órbita, descrita no final deste capítulo, utilizaremos o pacote VASP [29,30]. Logo é necessária uma abordagem téorica sobre a teoria do funcional da densidade (DFT - Density Functional Theory) [31]. A teoria do funcional da densidade foi desenvolvida em 1964 por Hohenberg e Kohn [32], implementada computacionalmente por John Pople [33]. Os dois últimos foram premiados com o Nobel em 1988. Parte do grande sucesso do DFT vem do avanço da aplicabilidade da teoria feita em 1965 por Kohn e Sham [34] graças às equações que levam seus nomes. 


\subsubsection{Descrição do problema}

Na seção anterior vimos que existe a necessidade de resolver a equação de Schrödinger da parte eletrônica que tem o formato da equação (2.14).

$$
\begin{gathered}
{\left[-\frac{\hbar^{2}}{2 m} \sum_{j}^{N} \nabla_{\vec{r}_{j}}^{2}-\frac{1}{4 \pi \epsilon_{0}} \sum_{i}^{M} \sum_{j}^{N} \frac{Z_{\overrightarrow{R_{i}}} e^{2}}{\left|\overrightarrow{r_{j}}-\overrightarrow{R_{i}}\right|}+\frac{1}{4 \pi \epsilon_{0}} \sum_{j}^{N} \sum_{l<j}^{N} \frac{e^{2}}{\left|\overrightarrow{r_{j}}-\overrightarrow{r_{l}}\right|}\right.} \\
\left.+\frac{1}{4 \pi \epsilon_{0}} \sum_{i}^{M} \sum_{k<i}^{M} \frac{Z_{\vec{R}_{i}} Z_{\vec{R}_{k}}^{2}}{e}\left|\overrightarrow{R_{i}}-\overrightarrow{R_{k}}\right|\right] \psi\left(\overrightarrow{r_{1}}, \overrightarrow{r_{2}}, \ldots, \overrightarrow{r_{N}}\right)=E_{m}(\vec{R}) \psi\left(\overrightarrow{r_{1}}, \overrightarrow{r_{2}}, \ldots, \overrightarrow{r_{N}}\right) .
\end{gathered}
$$

Uma das abordagens tradicionais para a solução da equação, é o método de HartreeFock [35], que é a base para cálculos mais refinados na química quântica. No método Hartree-Fock, parte-se do orbital molecular proposto a partir das idéias de Bohr, onde existe uma função de estado para cada elétron. A composição destas resulta na função de estado total dos $\mathrm{N}$ elétrons. Desta forma a função de onda total $\Psi$ é construída como sendo o produto anti-simétrico dos $N$ spin orbitais moleculares, de maneira a satisfazer o princípio de exclusão de Pauli. Podemos escrever esta função na forma de um único determinante de Slater [36,37] para sistemas de camada fechada, visto na equação $(2.15)$.

$$
\Psi=(N !)^{-\frac{1}{2}}\left|\begin{array}{cccc}
\psi_{1}^{1} & \psi_{2}^{1} & \cdots & \psi_{N}^{1} \\
\psi_{1}^{2} & \psi_{2}^{2} & \cdots & \psi_{N}^{2} \\
\vdots & \vdots & \vdots & \vdots \\
\psi_{1}^{N} & \psi_{2}^{N} & \cdots & \psi_{N}^{N}
\end{array}\right|
$$

onde $\psi_{i}^{\mu}$ são os spin orbitais moleculares (S.O.M), os índices $i$ representam o estado eletrônico, e $\mu$ as coordenadas do elétron. Como os S.O.M são linearmente independentes podemos sempre escolher uma matriz de transformação $\bar{A}$ tal que os $\psi_{i}^{\prime \mu}$ transformados formem um conjunto ortonormal $\int \psi_{k}^{*^{\prime} \mu} \psi_{l}^{\prime \mu} d^{3} r_{\mu}=\delta_{k l}$. Consequentemente teremos que:

$$
\int \Psi^{*} \Psi d r^{3}=1
$$

E assim obtemos o seguinte espectro de energia, com seu respectivo hamiltoniano:

$$
\begin{aligned}
E & =\int \Psi^{*} \hat{H} \Psi d r^{3}=E[\Psi], \\
\hat{H} & =\sum_{\mu} \hat{h}_{\mu}+\frac{1}{8 \pi \epsilon_{0}} \sum_{\mu \neq \nu} \frac{1}{r^{\mu \nu}}, \\
\hat{h}_{\mu} & =-\frac{\hbar^{2} \nabla^{2} \mu}{2 m}-\frac{1}{4 \pi \epsilon_{0}} \sum_{A} \frac{1}{r^{\mu A}},
\end{aligned}
$$


onde $\hat{h}_{\mu}$ é o operador hamiltoniano de uma partícula para o $\mu$-ésimo elétron movendo-se no campo dos núcleos, $\frac{\nabla^{2} \mu}{2}$ é a energia cinética do elétron $\mu$ e $\sum_{A} \frac{1}{r^{\mu A}}$ a energia coulombiana da interação elétron-núcleo. Ao desenvolver a energia em função do determinante de Slater obtemos para configurações de camadas fechadas, o espectro de Hartree da equação seguinte:

$$
\begin{aligned}
E(\Psi) & =2 \sum_{i} h_{i}+\frac{1}{4 \pi \epsilon_{0}} \sum_{i, j}\left(2 J_{i j}-K_{i j}\right), \\
h_{i} & =\int \psi_{i}^{* \mu} \hat{h}(\mu) \psi_{i}^{\mu} d^{3} r^{\mu}, \\
J_{i j} & =\int d^{3} r^{\nu} \int \psi_{i}^{* \mu} \psi_{j}^{* \nu} \psi_{i}^{\mu} \psi_{j}^{\nu} \frac{1}{r^{\mu \nu}} d^{3} r^{\mu}, \\
K_{i j} & =\int d^{3} r^{\nu} \int \psi_{i}^{* \mu} \psi_{j}^{* \nu} \psi_{j}^{\mu} \psi_{i}^{\nu} \frac{1}{r^{\mu \nu}} d^{3} r^{\mu},
\end{aligned}
$$

onde $J_{i j}$ representa a interação colombiana entre as densidades $\left|\psi_{i}\right|^{2}$ e $\left|\psi_{j}\right|^{2}$ e o termo $K_{i j}$ não tem análogo clássico e é conhecido como energia de "exchange" ou troca. Note que aqui reduzimos o problema de $\mathrm{N}$ elétrons para $\mathrm{N}$ problemas de um corpo. Além disso, nesta aproximação, cada partícula se move num campo médio, ou seja cada elétron participa do potencial que dá origem ao movimento de todos os elétrons. Isto significa que não há tratamento da correlação eletrônica. A inclusão da contribuição do movimento correlacionado é a principal preocupação dos métodos de primeiros princípios atuais. É comum definir a contribuição da energia de correlação como a diferença entre a energia exata não relativística e a energia de Hartree-Fock [38].

$$
E_{\text {corr }}=E_{\text {exata }}-E_{R H F},
$$

onde $E_{R H F}$ é a energia do método Hartree Fock restrito para camadas abertas uma extensão do método HF. Uma maneira alternativa ao método de Hartree-Fock é utilizar a densidade eletrônica total $\rho(r)$ como variável do sistema, nesta abordagem, as energias de troca e correlação são tratadas aproximadamente.

Segundo os Teoremas de Hohenberg e Kohn [32] a equação de Schrödinger de N elétrons, cuja variável básica era a função de onda com $3 \mathrm{~N}$ graus de liberdade, pode ter como nova variável a densidade eletrônica com apenas 3 graus de liberdade [39].

\subsection{Teoremas de Hohenberg-Kohn}

Dois teoremas são fundamentais para a Teoria do funcional da densidade. 
Teorema 2.3.1 O potencial externo $v(\vec{r})$ sentido pelos elétrons é um funcional único da densidade eletrônica $\rho(\vec{r})$.

Teorema 2.3.2 A energia do estado fundamental $E_{0}(n)$ é minima para a densidade $\rho_{0}(\vec{r})$.

Em outras palavras dada a densidade eletrônica do estado fundamental $\rho_{o}(\vec{r})$ é possível calcular a função de onda do estado fundamental correspondente $\psi_{0}\left(\overrightarrow{r_{1}}, \overrightarrow{r_{2}}, \ldots, \overrightarrow{r_{N}}\right)$, isto significa que $\psi_{0}$ é um funcional de $\rho_{o}(\vec{r})$. Consequentemente todos os observáveis são funcionais de $\rho_{o}(\vec{r})$ também. A função de onda e a densidade eletrônica contêm a mesma informação. Agora podemos escrever a energia no seguinte formato:

$$
\begin{aligned}
E[\rho] & =\min _{\psi \rightarrow \rho}\left\langle\psi\left|\hat{T}_{e}+\hat{V}_{e e}+\hat{V}_{N e}+\hat{V}_{N N}\right| \psi\right\rangle, \\
& =\left\langle\psi\left|\hat{T}_{e}+\hat{V}_{e e}\right| \psi\right\rangle+\int d^{3} r \rho(\vec{r}) v_{e x t}(\vec{r}), \\
& =F[\rho]+V_{e x t}[\rho] .
\end{aligned}
$$

Para usar estes teoremas, temos que obter as expressões analíticas de cada funcional descrito acima. É importante notar que $F[\rho]=\left\langle\psi\left|\hat{T}_{e}+\hat{V}_{e e}\right| \psi\right\rangle$ é um funcional universal e possui o mesmo formato para qualquer sistema que se queira estudar. Já $V_{\text {ext }}[\rho]=$ $\int d^{3} r \rho(\vec{r}) v_{\text {ext }}(\vec{r})$, depende da geometria do sistema, e qualquer força externa aplicada vai afetar exclusivamente este termo.

\subsection{Equações de Kohn-Sham}

Para obtermos a energia em função da densidade faremos uso das equações de KohnSham [34], onde iremos mapear o sistema de N elétrons interagentes, em um sistema de $\mathrm{N}$ elétrons não interagentes com a mesma densidade eletrônica. Escrevendo a energia e todos seus operadores como funcionais temos que:

$$
\left\langle\psi\left|\hat{H}_{e l e}\right| \psi\right\rangle \rightarrow E(\rho)=\hat{T}_{e}[\rho]+\hat{V}_{N e}[\rho]+\hat{V}_{N N}[\rho]+\hat{V}_{e e}[\rho]=\hat{T}[\rho]+\hat{U}[\rho]+\hat{V}_{e x t}[\rho] .
$$

Reescrevemos $\hat{U}[\rho]=\hat{V}_{e e}[\rho]$ e $\hat{V}_{e x t}[\rho]=\hat{V}_{N e}[\rho]+\hat{V}_{N N}[\rho]$. Até agora tudo é exato (dentro da aproximação de Born-Oppenheimer). Para termos fórmulas explícitas dos funcionais 
escrevemos o operador de energia cinética da seguinte forma:

$$
\hat{T}[\rho]=\hat{T}_{s}[\rho]+\hat{T}_{c}[\rho]=-\frac{\hbar^{2}}{2 m} \sum_{i}^{N} \int d^{3} \vec{r} \phi_{i}{ }^{*}(\vec{r}) \nabla^{2} \phi_{i}(\vec{r})+\hat{T}_{c}[\rho],
$$

onde $\hat{T}_{s}[\rho]$ é o termo de uma energia cinética de partícula não interagente no qual os $\phi_{i}(\vec{r})$ são orbitais de partícula única. Aqui vale notar que $\hat{T}_{s}$ não é um funcional explícito da densidade, e $\hat{T}_{c}[\rho]$ é um termo de correlação. Da mesma forma escrevemos a energia potencial na forma:

$$
\hat{U}[\rho]=\hat{U}_{H}[\rho]+\hat{U}_{x}[\rho]=\frac{e^{2}}{2\left(4 \pi \epsilon_{0}\right)} \int d^{3} \vec{r} \int d^{3} \overrightarrow{r^{\prime}} \frac{\rho(\vec{r}) \rho\left(\overrightarrow{r^{\prime}}\right)}{\left|\vec{r}-\overrightarrow{r^{\prime}}\right|}+\hat{U}_{x}[\rho]
$$

onde $\hat{U}_{H}[\rho]$ é o termo que descreve a interação de repulsão coulombiana clássica da nuvem eletrônica, descrita pela teoria de Hartree, e $\hat{U}_{x}[\rho]$ é um termo de troca, que poderia ser escrito explicitamente em função dos orbitais de partícula única devido ao princípio de exclusão de Pauli, mas veremos que no esquema de Kohn-Sham isto não é necessário.

Por último temos o potencial externo que pode ser escrito explicitamente $V_{e x t}[\rho]=$ $\int d^{3} \vec{r} v_{\text {ext }}(\vec{r}) \rho(\vec{r})$ o que, ao ser somado aos outros, resulta na equação (2.25).

$$
E[\rho]=T_{s}[\rho]+U_{H}[\rho]+V_{e x t}[\rho]+E_{x c}[\rho] .
$$

Todas as incertezas estão inseridas no termo de troca e correlação. De acordo com o Teorema variacional, tomando o mínimo de $E[\rho]$, com o vínculo que a carga total eletrônica seja fixa, como segue na seguinte equação:

$$
\int \rho(\vec{r}) d^{3} \vec{r}=N
$$

onde $N$ representa o número de elétrons, obtemos:

$$
\frac{\delta}{\delta \rho(\vec{r})}\left[E[\rho]-\mu\left(\int \rho(\vec{r}) d^{3} \vec{r}-N\right)\right]=0
$$

Aqui $\mu$ é um multiplicador de Lagrange, e tem sentido físico de potencial químico. Substituindo $E[\rho]$ na expressão (2.27) obtemos:

$$
\frac{\delta E[\rho]}{\delta \rho(\vec{r})}=\frac{\delta T_{s}[\rho]}{\delta \rho(\vec{r})}+\frac{\delta U_{H}[\rho]}{\delta \rho(\vec{r})}+\frac{\delta V_{e x t}[\rho]}{\delta \rho(\vec{r})}+\frac{\delta E_{x c}[\rho]}{\delta \rho(\vec{r})}=\mu
$$




$$
\frac{\delta T_{s}[\rho]}{\delta n(\vec{r})}+v_{e x t}(\vec{r})+v_{H}(\vec{r})+v_{x c}(\vec{r})=\mu
$$

Os três últimos termos do lado esquerdo da equação (2.29) podem ser vistos como potenciais. A idéia de Kohn-Sham, é introduzir um conjunto auxiliar de funções de onda de partícula única $\phi_{i}(\vec{r})$, para que este sistema gere a mesma densidade eletrônica que a função de onda de muitos corpos. Dado o potencial $v_{s}(\vec{r})=v_{\text {ext }}(\vec{r})+v_{H}(\vec{r})+v_{x c}(\vec{r})$, obtemos a mesma densidade que o problema de interesse, onde agora basta resolver a equação (2.30) de elétrons não interagentes.

$$
\left[\frac{-\hbar^{2} \nabla^{2}}{2 m}+v_{s}(\vec{r})\right] \phi_{i}(\vec{r})=e_{i} \phi_{i}(\vec{r})
$$

Assim, podemos reproduzir a densidade $\rho(\vec{r})$, pela equação:

$$
\rho(\vec{r})=\sum_{i}^{N} f_{i}\left|\phi_{i}(\vec{r})\right|^{2}
$$

onde os $f_{i}$, representam a ocupação de cada orbital. Lembrando que $v_{H}(\vec{r})$ e $v_{x c}(\vec{r})$ dependem de $\rho(\vec{r})$, que depende dos orbitais $\phi_{i}(\vec{r})$, que por sua vez depende de $v_{s}(\vec{r})$, o problema tem que ser resolvido autoconsistentemente. Note que a equação de Kohn-Sham toma o formato da equação (2.32).

$$
\left(-\frac{\hbar^{2}}{2 m} \nabla^{2}+v_{\text {ext }}(\vec{r})+\frac{e^{2}}{4 \pi \epsilon_{0}} \int \frac{\rho(\vec{r})}{\left|\vec{r}-\overrightarrow{r^{\prime}}\right|}+v_{x c}(\rho)\right) \psi_{i}=e_{i} \psi_{i} .
$$

Ao mutiplicar à esquerda por $\psi_{i}^{*}$, integrar no espaço das coordenadas e somar sobre todos os orbitais ficamos com a equação:

$$
\sum_{i}^{N} e_{i}=T_{s}(\rho)+\int v_{\text {ext }} \rho(\vec{r}) d^{3} \vec{r}+\frac{e^{2}}{4 \pi \epsilon_{0}} \iint \frac{\rho(\vec{r}) \rho\left(\overrightarrow{r^{\prime}}\right)}{\left|\vec{r}-\overrightarrow{r^{\prime}}\right|} d^{3} \vec{r} d^{3} \vec{r}^{\prime}+\int v_{x c}(\rho) \rho(\vec{r}) d^{3} \vec{r},
$$

comparando com (2.25), ficamos com a seguinte energia total:

$$
E_{0}=\sum_{i}^{N} e_{i}-\frac{e^{2}}{2\left(4 \pi \epsilon_{0}\right)} \iint \frac{\rho_{0}(\vec{r}) \rho_{0}\left(\overrightarrow{r^{\prime}}\right)}{\left|\vec{r}-\overrightarrow{r^{\prime}}\right|} d^{3} \vec{r} d^{3} \vec{r}^{\prime}-\int v_{x c}(\vec{r}) \rho_{0}(\vec{r}) d^{3} \vec{r}+E_{x c}\left[\rho_{0}\right] .
$$

Vemos que as soluções numéricas das equações de Kohn e Sham se resumem a expandir os orbitais em uma base apropriada, e escolher uma boa aproximação para a energia de troca e correlação. 


\subsubsection{Escolha da Base}

Há dois tipos de base, bases fixas que não dependem da energia, e as dependentes da energia. As bases fixas na energia são aquelas usadas nas expansões em ondas planas (Caso do VASP), no método de aproximação Tight Binding ou LCAO (Linear Combination of Atomic Orbitals) [40], ou mesmo nos métodos OPW (Orthogonalized Plane Wave) [41]. Exemplos de bases dependentes da energia são APW (Augmented Plane Wave) [42], ou método KKR (Korringa-Kohn-Rostoker) [43,44], que não serão discutidos aqui.

No método de bases fixas, será necessário resolver a equação de autovalores do hamiltoniano. A vantagem é ser computacionalmente mais simples, a desvantagem é que a base tem que ser muito grande para ser completa.

\subsubsection{Escolhas para o termo $E_{x c}[\rho]$}

O formato exato para o funcional de troca e correlação não existe, todavia há duas escolhas que se destacam na computação de primeiros princípios. A primeira é a aproximação LDA (Local Density Approximation). Nessa aproximação a densidade varia suavemente nas proximidades de $\vec{r}$, é utilizada a energia de troca-correlação de um líquido de elétrons distribuídos homogeneamente.

$$
E_{x c}^{L D A}=\frac{e^{2}}{4 \pi \epsilon_{0}} \int d^{3} \vec{r} e_{x c}^{h o m}(\rho(\vec{r}))=\frac{e^{2}}{4 \pi \epsilon_{0}} \int d^{3} \vec{r}\left(e_{x}^{h o m}+e_{c}^{h o m}\right)(\rho(\vec{r})) .
$$

Nesta aproximação separa-se o termo de troca e correlação, em um termo de troca que é conhecido exatamente pela equação de Dirac:

$$
e_{x}^{h o m-L D A}[\rho]=\frac{-3}{4}\left(\frac{3}{\pi}\right)^{\frac{1}{3}} \rho^{\frac{4}{3}}
$$

e ainda um termo de correlação que não pode ser determinado exatamente, porém pode ser parametrizado pelos cálculos feitos por Ceperley e Alder [45].

Sabendo-se que qualquer sistema real tem uma densidade eletrônica variável no espaço, espera-se a inclusão de termos que levem em conta a taxa de variação da densidade no espaço. Para isto temos a aproximação GGA (Generalized Gradiant Approximation). Nessa aproximação a energia é expressa em termos do gradiente da densidade da carga 
total.

$$
E_{x c}^{G G A}=\frac{e^{2}}{4 \pi \epsilon_{0}} \int d^{3} \vec{r} f((n(\vec{r})), \nabla(n(\vec{r}))) .
$$

Diferentes GGAs diferem pelo formato da função $f((n(\vec{r})), \nabla(n(\vec{r}))$. As propostas para este segundo método levam o nome de seus criadores, PBE (Perdew - Burke - Erzenhot) [46]; LYPB (Lee - Yang - Parr - Becke) [47,48]; PW (Perdew - Wang) [49]; entre outras.

De maneira geral os GGAs atuais proporcionam resultados muito confiáveis para todo tipo de ligação química (covalente, iônica, metálica e pontes de hidrogênio), apesar de não descreverem muito bem interações de Van der Walls. Hoje em dia o GGA mais popular é o PBE proposto em 1996 por Perdew - Burke - Erzenhot. Para se usar os conceitos de DFT e calcular propriedades elétricas, térmicas, óticas ou magnéticas é necessário estudar sistemas periódicos, onde graças ao teorema de Bloch os cálculos tornam-se muito mais simples.

\subsection{Sistemas periódicos e Teorema de Bloch}

Um cristal ideal é construído pela repetição infinita no espaço de unidades estruturais idênticas. Em física do estado sólido dizemos que os cristais são definidos por uma rede de Bravais e por uma base de átomos anexadas identicamente a cada ponto desta rede. A rede de Bravais é um sistema de infinitos pontos com arranjos regulares em todo o espaço, esta rede possui simetria translacional, onde dado dois pontos quaisquer da rede $\vec{r}$ e $\vec{r}$ podemos construir um conjuto de vetores primitivos, que nos permite montar um vetor de translação, visto na seguinte equação:

$$
\begin{gathered}
\vec{r}=\vec{r}^{\prime}+\vec{R}_{n}, \\
\vec{R}_{n}=\sum_{i=1,2,3} l_{n i} \vec{a}_{i},
\end{gathered}
$$

com $l_{n i}(i=1,2,3)$ sendo inteiros. Como os íons em um cristal perfeito estão posicionados de maneira periódica, o potencial tem a mesma periodicidade, ou seja:

$$
V\left(\vec{r}+\vec{R}_{n}\right)=V(\vec{r}) .
$$

Mesmo sabendo que o problema eletrônico em um sólido é, em princípio, um problema de muitos elétrons, onde para o hamiltoniano temos termos de interação elétron-elétron 
e elétron-núcleo, no esquema de Kohn-Sham todos os elétrons estão sujeitos ao mesmo potencial (idéia da aproximação de elétrons independentes). Sabemos que elétrons que obedecem a equação (2.40) com um potencial periódico, são conhecidos como elétrons de Bloch.

$$
\left[\frac{-\hbar^{2} \nabla^{2}}{2 m}+v_{s}(\vec{r})\right] \phi_{i}(\vec{r})=e_{i} \phi_{i}(\vec{r})
$$

e obedecem ao seguinte Teorema de Bloch:

Teorema 2.5.1 As autofunções $\psi$ de um hamiltoniano monoeletrônico $H=\frac{-\hbar^{2} \nabla^{2}}{2 m}+$ $V(\vec{r})$, onde $V\left(\vec{r}+\vec{R}_{n}\right)=V(\vec{r})$ para todos os $\vec{R}_{n}$ em uma rede de bravais, pode ser escolhido como uma onda plana vezes uma função com a periodicidade da mesma rede de Bravais:

$$
\begin{gathered}
\phi_{\vec{k}}(\vec{r})=e^{i \vec{k} \cdot \vec{r}} u_{\vec{k}}(\vec{r}), \\
u_{\vec{k}}\left(\vec{r}+\vec{R}_{n}\right)=u_{\vec{k}}(\vec{r}) .
\end{gathered}
$$

Note que com o auxílio do teorema de Bloch podemos transformar $H_{K S}$ em $H_{k}=$ $e^{-i \vec{k} \cdot \vec{r}} H_{K S} e^{i \vec{k} \cdot \vec{r}}$. Isto nos leva à seguinte equação de Schrödinger no espaço recíproco:

$$
H_{k} u_{\vec{k}}(\vec{r})=\left[\frac{\hbar^{2} k^{2}}{2 m}+v_{s}(\vec{r})\right] u_{\vec{k}}(\vec{r}) .
$$

Assim os autovalores $e_{\vec{k}}$ e as autofunções $\phi_{\vec{k}}(\vec{r})$ dos estados de Kohn-Sham, são então, classificados através dos vetores de onda $\vec{k}$.

\subsubsection{Rede Recíproca e Zona de Brillouin}

Em princípio teríamos que calcular os autovalores e autovetores de Kohn-Sham no formalismo de Bloch para infinitos vetores $\vec{k}$. Veremos que isso não é necessário ao se definir rede recíproca e zona de Brillouin.

Uma rede recíproca associada à rede cristalina (definida pela rede de Bravais), pode ser construída a partir de vetores $\vec{G}_{m}$ obedecendo a relação:

$$
\vec{G}_{m} \cdot \vec{R}_{n}=2 \pi \times \text { inteiro }
$$


Da mesma forma que na rede direta, os vetores $\vec{G}_{m}$, são vetores de translação na rede recíproca, onde também podem ser expressos em função de vetores primitivos:

$$
\vec{G}_{m}=\sum_{i=1,2,3} g_{m i} \vec{b}_{i}
$$

Para que a relação $(2.44)$ seja satisfeita os vetores $\overrightarrow{b_{i}}$ devem satisfazer a condição:

$$
\vec{a}_{i} \cdot \vec{b}_{j}=2 \pi \delta_{i j}
$$

Para isto basta escolher os vetores da seguinte forma:

$$
\begin{aligned}
& \vec{b}_{1}=2 \pi \frac{\vec{a}_{2} \times \vec{a}_{3}}{\vec{a}_{1} \cdot\left(\vec{a}_{2} \times \vec{a}_{3}\right)}, \\
& \vec{b}_{2}=2 \pi \frac{\vec{a}_{3} \times \vec{a}_{1}}{\vec{a}_{1} \cdot\left(\vec{a}_{2} \times \vec{a}_{3}\right)}, \\
& \vec{b}_{3}=2 \pi \frac{\vec{a}_{1} \times \vec{a}_{2}}{\vec{a}_{1} \cdot\left(\vec{a}_{2} \times \vec{a}_{3}\right)} .
\end{aligned}
$$

Agora para construir uma célula unitária (Célula primitiva de menor volume) da rede recíproca, devido à arbitrariedade de escolha adotamos que seja uma célula de WignerSeitz [50]. Esta célula é construída em torno de um ponto da rede desenhando linhas que conectam cada ponto da rede e então bisectando com um plano cada uma dessas linhas, ao final pegamos o menor poliedro contendo os pontos ligados por estes planos. Esta célula possui todas as simetrias da rede recíproca e chama-se primeira zona de Brillouin. Pela construção notamos que $\vec{k}^{\prime}$ e $\vec{k}$ são pontos equivalentes se:

$$
\vec{k}^{\prime}=\vec{k}+\vec{G}
$$

Neste caso $e^{i \vec{k}^{\prime} \cdot \vec{R}_{n}}=e^{i \vec{k} \cdot \vec{R}_{n}}$ devido a $e^{i \vec{G} \cdot \vec{R}_{n}}=1$, logo as funções de onda $\phi_{\vec{k}^{\prime}}(\vec{r})$ e $\phi_{\vec{k}}(\vec{r})$ satisfazem as mesmas condições de contorno, portanto, possuem o mesmo autovalor, apesar de poderem representar diferentes funções de onda. Podemos então nos restringir a calcular os autovalores de Kohn-Sham apenas na região obedecida pela condição:

$$
|\vec{k}| \leq|\vec{k}+\vec{G}|
$$

Tal esquema é chamado de redução à primeira zona de Brillouin, onde $e_{k}$ torna-se uma função multivalente de $\vec{k}$, ou seja agora temos $e_{n \vec{k}}$. Quando estamos trabalhando com condições periódicas de contorno os orbitais de Kohn-Sham, além de satisfazer o Teorema 
de Bloch, são avaliados para todos os pontos $\mathrm{k}$ da primeira zona de Brillouin em todas as bandas. A densidade de carga neste formalismo é dada por:

$$
\rho(\vec{r})=\sum_{n \vec{k}} f_{n \vec{k}}\left|\phi_{n \vec{k}}(\vec{r})\right|^{2},
$$

onde $f_{n \vec{k}}$ é a ocupação de banda $n$ no ponto $\vec{k}$ da rede recíproca. Na prática a soma pode ser aproximada por um número pequeno de pontos. Estes últimos são construídos dentro da zona de Brillouin irredutível que corresponde ao menor volume dentro da primeira zona que a cobre inteiramente quando operamos todas as operações de simetria do grupo em questão.

Vários esquemas para a escolha de pontos na rede recíproca são propostos, no de Baldereschi [51] escolhe-se um único, dito melhor ponto $\vec{k}$. Outro método seria o de Chadi e Cohen [52], porém um dos mais utilizados, inclusive aqui na tese é o de Monkhorst e Pack [53]. Para metais onde a superfície de Fermi é mais delicada usamos pontos igualmente espaçados em torno do ponto $\vec{k}=0$ correspondente ao ponto $\Gamma$, incluído o próprio.

\subsubsection{Condição de contorno cíclica}

Sabemos que um cristal real é finito, mesmo tendo um número de células unitárias muito grande. A condição de contorno cíclica, introduzida por Born Von Karman, permite um tratamento matemático para esses sistemas. Agora, nossos vetores da equação 2.38 estão restringidos pelas seguintes equações:

$$
0<=l_{n i}<N_{i} \quad i=1,2,3,
$$

onde $N=\prod_{i} N_{i}$ é o número de células unitárias no sistema real. A condição de contorno cíclica é dada por:

$$
\phi_{\vec{k}}\left(\vec{r}+N_{i} \vec{a}_{i}\right)=\phi_{\vec{k}}(\vec{r}) .
$$

Esta equação impõe restrições nos autovetores de Bloch, ou seja $e^{i\left(\vec{k} \cdot N_{i} \vec{a}_{i}\right)}=1$. Como consequência $\vec{k}$ assume os seguinte valores:

$$
\vec{k}=\sum_{j=1}^{3} \frac{m_{j}}{N_{j}} \vec{b}_{j},
$$


onde $m_{j}$ é um inteiro. Cada vez que $m_{j}$ muda de um temos um novo autoestado $\phi_{\vec{k}}$. Logo o volume no espaço recíproco contendo apenas um único estado é dado por:

$$
V_{\text {estado }}=\frac{\vec{b}_{1}}{N_{1}} \cdot\left(\frac{\vec{b}_{2}}{N_{2}} \times \frac{\vec{b}_{3}}{N_{3}}\right)=\frac{V_{B Z}}{N} .
$$

Esta equação nos mostra que o volume da zona de Brillouin $V_{B Z}$ ocupada, contém o mesmo número de estados $\phi_{\vec{k}}$ quanto número de células primitivas do cristal. Lembrando que ao se tratar de férmions, devido ao princípio de exclusão de Pauli, cada estado pode acomodar duas polarização de spin distintas. Então cada banda em uma zona de Brillouin pode conter $2 \mathrm{~N}$ estados. Esta propriedade irá determinar de que maneira ocupamos as bandas de energia eletrônica do material, definindo se o sistema é isolante, semicondutor, metal entre outros.

\subsubsection{Método de ondas planas}

Calcular as autofunções e autovalores da equação de Schrödinger para sistemas periódicos é um problema de interesse desde o início da mecânica quântica. Para sistemas periódicos, graças ao teorema de Bloch, a função $u_{n \vec{k}}(\vec{r})$ pode ser expandida em ondas planas de forma obtermos $\phi_{n \vec{k}}(\vec{r})=\sum_{\vec{G}} C_{\vec{k}+\vec{G}} e^{i(\vec{k}+\vec{G}) \cdot \vec{r}}$. Substituindo no Hamiltoniano de Kohn Sham (equações auxiliares) obtemos:

$$
\left[\frac{-\hbar^{2}}{2 m} \nabla^{2}+v_{s}\right]\left(\sum_{\vec{G}} C_{\vec{k}+\vec{G}} e^{i(\vec{k}+\vec{G}) \cdot \vec{r}}\right)=e_{n \vec{k}} \sum_{\vec{G}} C_{\vec{k}+\vec{G}} e^{i(\vec{k}+\vec{G}) \cdot \vec{r}}
$$

Agora multiplicando a equação acima por $e^{-i(\vec{k}+\vec{G}) \cdot \vec{r}}$ à esquerda e integrando na primeira zona de Brillouin, ficamos com:

$$
\left[\frac{-\hbar^{2}}{2 m}(\vec{k}+\vec{G})^{2}-e_{n \vec{k}}\right] C_{\vec{k}+\vec{G}}+\sum_{G^{\prime}} V_{\vec{G}-\vec{G}^{\prime}}^{s} C_{\vec{k}+\vec{G}}=0
$$

com o potencial escrito da seguinte forma:

$$
V_{\vec{G}-\vec{G}^{\prime}}^{s}=\int_{\text {ZonaBrillouin }} v_{s} e^{i\left(\vec{G}-\vec{G}^{\prime}\right) \cdot \vec{r}} d v
$$

Assim obtemos os autovalores e autovetores da equação (2.56). Quanto maior o número de ondas planas melhor será a descrição do problema, a quantidade de ondas utilizadas 
é estipulada em função de uma energia de corte em elétron-volts. Todas as ondas planas com uma energia cinética menor que $E_{c u t}$ serão incluídas na base, isto é $|\vec{G}+\vec{k}|<G_{c u t}$ onde $E_{c u t}=\frac{\hbar^{2}}{2 m} G^{2}$ cut .

A base em ondas planas é vantajosa por ser uma base completa, matematicamente simples, e que suas derivadas tornam-se produtos no espaço recíproco, além de não depender das posições dos átomos. Porém além da necessidade de se descrever o vácuo, eventualmente podem existir problemas de convergência devido aos estados dos elétrons de caroço, que apresentam oscilações muito rápidas em função do espaço e consequentemente suas descrições apropriadas exigem muitas ondas planas. Este último problema pode ser contornado com o uso do método do Pseudopotencial.

\subsubsection{Método de base localizada}

Uma outra forma de descrever as funções de Bloch é utilizar um conjunto de funções base localizadas sobre os sítios atômicos. Este método inserido no código SIESTA (Spanish Initiative for Electronic Simulations with Thousands of Atoms) [54] e no código OPENMX (OPEN source package for Material eXplorer) [55], é um dos mais utilizados na física da matéria condensada, pois permite o estudo de sistema com milhares de átomos por um menor custo computacional.

Neste método podemos assumir que na vizinhança de cada ponto da rede direta o hamiltoniano total pode ser aproximado por um hamiltoniano de sítio local periódico. Se o sistema tem N sítios, onde cada um deles possui uma função de onda associada, a autofunção do hamiltoniano total será uma combinação linear destas N autofunções de um sítio.

$$
\phi_{n \vec{k}}(\vec{r})=\sum_{\vec{R}_{n}} \omega_{n \vec{k}}\left(\vec{r}-\vec{R}_{n}\right) e^{i \vec{R}_{n} \cdot \vec{k}},
$$

onde $\omega_{n \vec{k}}\left(\vec{r}-\vec{R}_{n}\right)$ são as funções de Wanniers centradas em cada ponto da rede, nota-se que estas funções têm a mesma periodicidade da rede.

Agora utilizando um conjunto base de funções atômicas centradas em cada átomo localizados em $\vec{R}_{n}+\vec{\tau}_{\alpha}$ (aqui temos $\vec{\tau}_{\alpha}$ que leva em conta pontos da rede de Bravais que 
contenham mais de que um átomo).

$$
\omega_{n \vec{k}}\left(\vec{r}-\vec{R}_{n}\right)=\sum_{\mu, \alpha} C_{i \mu}(\vec{k}) \chi_{\mu}\left(\vec{r}-\vec{\tau}_{\alpha}-\vec{R}_{n}\right)
$$

Substituindo na hamiltoniana de Kohn-Sham, obtemos:

$$
\begin{gathered}
\sum_{\mu, \alpha}\left[\sum_{\vec{R}_{n}} e^{i \vec{R}_{n} \cdot \vec{k}}\left(\frac{\hbar^{2}}{2 m} \nabla^{2}+V_{s}(\vec{r})\right) \chi_{\mu}\left(\vec{r}-\vec{\tau}_{\alpha}-\vec{R}_{n}\right)\right] C_{i \mu}(\vec{k}), \\
=e_{i}(\vec{k}) \sum_{\mu, \alpha} \sum_{\vec{R}_{n}} e^{i \vec{R}_{n}} \chi_{\mu}\left(\vec{r}-\vec{\tau}_{\alpha}-\vec{R}_{n}\right) C_{i \mu}(\vec{k}) .
\end{gathered}
$$

Multiplicando a equação (2.60) por uma função base $\chi_{\nu}^{*}\left(\vec{r}-\vec{\tau}_{\alpha}\right)$ e integrando no espaço real, ficamos com:

$$
\left.\sum_{\mu}\left[H_{\nu \mu}(\vec{k})-e_{n \vec{k}}\right) S_{\nu \mu}(\vec{k})\right] C_{i \mu}(\vec{k})=0
$$

onde temos:

$$
\begin{gathered}
H_{\nu \mu}(\vec{k})=\sum_{\vec{R}_{n}, \alpha} e^{i \vec{R}_{n} \cdot \vec{k}}\left[\int d^{3} r \chi^{*}{ }_{\nu}\left(\vec{r}-\vec{\tau}_{\alpha}\right)\left(H_{K S}\right) \chi_{\mu}\left(\vec{r}-\vec{\tau}_{\alpha}-\vec{R}_{n}\right)\right], \\
S_{\nu \mu}(\vec{k})=\sum_{\vec{R}_{n} i, \alpha} e^{i \vec{R}_{n} \cdot \vec{k}}\left[\int d^{3} r \chi^{*}{ }_{\nu}\left(\vec{r}-\vec{\tau}_{\alpha}\right) \chi_{\mu}\left(\vec{r}-\vec{\tau}_{\alpha}-\vec{R}_{n}\right)\right] .
\end{gathered}
$$

Assim obtemos os autovalores e autovetores da equação (2.61). As vantagens de bases localizadas se apoiam no fato da simples interpretação física no formato Tigh Binding, além de necessitar de uma base bem menor que a de ondas planas, o problema aparece no fato desta base não ser ortogonal e depender das posições atômicas.

\subsection{Método dos pseudopotenciais}

Para resolver a equação de Kohn-Sham para sistemas de muitos átomos usa-se do método do Pseudopotencial. Neste formalismo que cresceu em torno do método OPW (Ortogonalized Planes Waves) construído por Phillips e Kleinman [56], propõe-se uma expansão envolvendo uma combinação linear de estados de caroço de forma a reduzir o tempo computacional.

No método a idéia é separar os orbitais de caroço dos de valência, pois todas as informações sobre propriedades eletrônicas estão presentes nesta última. Então as funções de onda são projetadas em um espaço ortogonal ao espaço dos orbitais de caroço, com o uso do projetor:

$$
\hat{P}=\sum_{c}=\left|\phi_{c}\right\rangle\left\langle\phi_{c}\right|
$$


Assim o orbital para um dado estado eletrônico será um combinação de estados ortogonais ao caroço vista na seguinte equação:

$$
\left|\phi_{n \vec{k}}\right\rangle=(1-\hat{P}) \sum_{\vec{G}} C_{\vec{k} \vec{G}}|\vec{k}+\vec{G}\rangle
$$

Ao subtituir na equação de Schrödinger onde a base não esta projetada $\hat{H}\left|\phi_{n \vec{k}}\right\rangle=e_{n \vec{k}}\left|\phi_{n \vec{k}}\right\rangle$, obtemos:

$$
\left(\hat{H}+\sum_{c}\left(e_{n \vec{k}}-e_{c}\right)\left|\phi_{c}\right\rangle\left\langle\phi_{c}\right|\right)|\vec{k}+\vec{G}\rangle=\left(\hat{H}+\hat{V}_{R}\right)|\vec{k}+\vec{G}\rangle=e_{n \vec{k}}|\vec{k}+\vec{G}\rangle
$$

Notamos que a equação acima tem os mesmos autovalores que a equação não projetada, porém, o formato do lado esquerdo mudou. Podemos escrever na equação $(2.65),\left|\phi_{n \vec{k}}\right\rangle=$ $(1-\hat{P})\left|\phi_{n \vec{k}}^{P S}\right\rangle$, onde $\left|\phi_{n \vec{k}}^{P S}\right\rangle$ é uma pseudofunção. Ao substituir a hamiltoniana por $\hat{H}=\frac{-\hbar^{2} \nabla^{2}}{2 m}+\hat{V}$ obtemos:

$$
\left(\frac{-\hbar^{2} \nabla^{2}}{2 m}+\hat{V}+\hat{V}_{R}\right)\left|\phi_{n \vec{k}}^{P S}\right\rangle=\left(\frac{-\hbar^{2} \nabla^{2}}{2 m}+\hat{V}_{P S}\right)\left|\phi_{n \vec{k}}^{P S}\right\rangle
$$

onde $\hat{V}_{P S}$ é o pseudopotencial. O uso de pseudopotenciais tem provado ser um importante passo em métodos de primeiros princípios para modelar sistemas grandes. A idéia é que elétrons próximos ao núcleo permanecem localizados independentemente do ambiente químico, ou seja ao invés de utilizar o potencial Coulombiano $-\frac{Z e}{\left(4 \pi \epsilon_{0}\right) r}$ para descrever interações nucleares usamos um pseudopotencial $V_{p s}(r)$ que elimina os estados de caroço dos cálculos. Para a construção de Pseudopotenciais, algumas aproximações estão implicitamente usadas:

- Quando dividimos elétrons de caroço e de valência assumimos a idéia de elétron independente, que está naturalmente inserida no esquema de Kohn-Sham.

- Inserimos também a frozen core approximation que significa que alguns estados monoeletrônicos não mudam significamente, ou seja eles permanecem congelados quando passamos de um ambiente químico para outro.

- Assumimos que não existe overlap significativo entre funções de onda de caroço e de valência, o que não é verdade para elétrons do tipo $d$ e $f$. 
Dadas essas aproximações o grande desafio na elaboração de pseudopotenciais era a transferibilidade de um sistema, para outro, uma vez que no começo das elaborações eram feitas de modo empírico. Houve grande sucesso no início dos anos 80 com a invenção de pseudopotenciais de norma conservada. Hoje em dia os pseudopotenciais Ab Initio de norma conservada nos desenvolvimentos de Bachelet, Hamann e Schluter (BHS) [57] e de Troullier-Martins [58], são os mais utilizados. Nesses métodos os pseudopotenciais obedecem às seguintes regras:

- Os autovalores $e_{i}$ obtidos para os estados de valência atômicos devem ser, por construção, idênticos aos autovalores $e_{i}^{P S}$ obtidos com o pseudopotencial.

- As autofunções relativas à autofunção exata, dita função all electron, e à solução obtida com o pseudopotencial devem ser iguais para $r>r_{c}$, onde $r_{c}$ é um raio de corte.

- As integrais de 0 a $r$, para $r>r_{c}$ das densidades de carga da autofunção exata devem ser iguais às das soluções obtidas com o pseudopotencial.

- A derivada logarítmica da pseudofunção deve convergir para a da autofunção exata para $r>r_{c}$

O método de obtenção desses pseudopotencias de norma conservada é feita da seguinte forma:

1. Resolve-se a equação de Dirac para um átomo isolado de forma a obter a função de onda $P_{l}(r)$ exata, onde todos os elétrons são considerados.

2. A primeira etapa do pseudopotencial é construída retirando a singularidade no ponto $r=0$ do potencial all electron achado acima, de maneira que os autovalores não sejam mudados.

3. Os pseudo-orbitais construídos deste novo potencial são modificados de forma a obedecer conservação de norma, até um raio de corte $r_{c}$ escolhido entre o último nó e pico da função de onda, delimitando a divisão entre caroço e valência, além 
de reproduzir a função all electron depois deste raio de corte, visto nas seguintes equações.

$$
\begin{gathered}
\int_{0}^{r_{c}} r^{2} d r P_{p s}^{l}(r)=\int_{0}^{r_{c}} r^{2} d r P^{l}(r), \\
P_{p s}^{l}=\left\{\begin{array}{rll}
f(r) & \text { for } & r<r_{c} \\
P_{l}(r) & \text { for } & r>r_{c}
\end{array}\right.
\end{gathered}
$$

onde $f(r)$ é uma função parametrizada contínua, cuja derivada também é continua.

4. A segunda etapa do pseudopotencial é construir o potencial que gera as funções de onda acima, via inversão da equação de Schrödinger radial, ou seja dada equação abaixo:

$$
\left(\frac{-1}{2} \frac{d^{2}}{d r^{2}}+\frac{l(l+1)}{2 r^{2}}+V_{p s}^{l}(r)\right) P_{p s}^{l}(r)=e_{l} P_{p s}^{l}(r)
$$

Invertendo temos:

$$
V_{p s}^{l}=e_{l}-\frac{l(l+1)}{2 r^{2}}+\frac{1}{2} \frac{P_{p s}^{\prime l}(r)}{P_{p s}^{l}(r)} .
$$

5. Finalmente para transferir esta pseudofunção para outros ambientes químicos temos que substrair as informações relativas a potencial de troca e correlação além do termo de Hartree, calculados com a pseudo densidade de carga $\tilde{\rho}$.

$$
\tilde{V}_{p s}^{l}(r)=V_{p s}^{l}(r)-v_{x c}[\tilde{\rho}(r)]-v_{H}[\tilde{\rho}(r)]
$$

Ficamos com o seguinte potencial externo:

$$
V_{e x t}=\sum_{i}^{M} \sum_{k<i}^{M} \frac{e^{2}}{2\left(4 \pi \epsilon_{0}\right)} \frac{Z_{\vec{R}_{i}} Z_{\vec{R}_{k}}}{\left|\vec{R}_{i}-\vec{R}_{k}\right|}+\sum_{i l m}\left|Y_{l m}\right\rangle \tilde{V}_{p s}^{l}\left(\left|\vec{r}-\vec{R}_{i}\right|\right)\left\langle Y_{l m}\right| .
$$

\subsection{Método PAW}

Em simulação computacional, quando o foco é estudar metais de transição, onde elétrons $d$ e $f$, além dos estados de semi-caroço passam a ser importantes, o tratamento apenas com pseudopotencial, fica comprometido. O comportamento dessas regiões é intensamente oscilatório exigindo uma quantidade muito grande de ondas planas. No método do pseudopotencial perdemos informação da função de onda total próxima ao núcleo e isto pode influenciar nas propriedades eletrônicas. 
Um método all electron proposto por Peter Blochl [59] (e implementado no código VASP [60]) que leva em conta tais contribuições de caroço chama-se PAW (Projected Augmented Waves). A idéia é dividir o espaço em esferas centradas nos átomos, e outra região fora destas esferas. Dentro das esferas o formato da função de onda será a de ondas atômicas parciais, fora das esferas serão definidas funções de cobertura suave, e nas bordas das esferas tais funções serão conectadas. Vamos agora explicar brevemente o método e as aproximações que estão por trás desta idéia.

Para nos livrar das oscilações indesejadas da função de onda próxima ao núcleo, procuramos uma transformação linear $\hat{T}$ que nos leve de uma função auxiliar suave $\left|\tilde{\psi}_{n}\right\rangle$ para a função de onda all electron de Kohn-Sham $\left|\psi_{n}\right\rangle$, onde $n$ é um índice que agrega índices de ponto $\vec{k}$, de banda e de spin.

$$
\left|\psi_{n}\right\rangle=\hat{T}\left|\tilde{\psi}_{n}\right\rangle
$$

Agora teremos que resolver a nova equação de autovalores e autovetores de Kohn-Sham:

$$
\hat{T}^{\dagger} \hat{H}_{K S} \hat{T}\left|\tilde{\psi}_{n}\right\rangle=e_{n} \hat{T}^{\dagger} \hat{T}\left|\tilde{\psi}_{n}\right\rangle
$$

O trabalho se resume em escolher este operador de transformação apropriadamente. Dado que as funções de onda naturalmente já são suaves a partir de uma certa distância do núcleo, temos que:

$$
\hat{T}=1+\sum_{a} \hat{T}_{a}
$$

onde $a$ é um índice de núcleo. $\hat{T}_{a}$ não tem nenhum efeito fora da região da esfera ou seja para $\left|\vec{r}-\vec{R}_{a}\right|<r_{c}^{a}$, onde $r_{c}^{a}$ é uma raio de corte de maneira a não existir overlap entre as esferas aumentadas. Dentro das esferas aumentadas expandimos a função de onda all electron em ondas parciais $\phi_{i}^{a}$, onde, para cada onda parcial, definimos uma função auxiliar suave correspondente $\tilde{\phi}_{i}^{a}$, logo analogamente à equação (2.73), temos:

$$
\left|\phi_{i}^{a}\right\rangle=\left(1+\hat{T}_{a}\right)\left|\tilde{\phi}_{i}^{a}\right\rangle \Longleftrightarrow \hat{T}_{a}\left|\tilde{\phi}_{i}^{a}\right\rangle=\left|\phi_{i}^{a}\right\rangle-\left|\tilde{\phi}_{i}^{a}\right\rangle .
$$

Isto define o operador linear $\hat{T}$, dados $\phi$ e $\tilde{\phi}$. Se agora as ondas parciais suaves formam uma base completa dentro da esfera aumentada, podemos expandir a função all electron 
suavisada para $\left|\vec{r}-\vec{R}_{a}\right|<r_{c}^{a}$ como:

$$
\left|\tilde{\psi}_{n}\right\rangle=\sum_{i} P_{n i}^{a}\left|\tilde{\phi}_{i}^{a}\right\rangle
$$

onde $P_{n i}^{a}$ são coeficientes da expansão. Mas como $\left|\phi_{i}^{a}\right\rangle=\hat{T}\left|\tilde{\phi}_{i}^{a}\right\rangle$, obtemos o seguinte formato para a função all electron total:

$$
\left|\psi_{n}\right\rangle=\sum_{i} P_{n i}^{a}\left|\phi_{i}^{a}\right\rangle
$$

Ambas as funções de onda all electron suave e total têm os mesmos coeficientes de expansão. Como não há overlap entre as esferas aumentadas, podemos escolher as funções de projeção suave de forma a satisfazer ortogonalidade e completeza.

$$
\begin{gathered}
\left\langle\tilde{P}_{i}^{a} \mid \tilde{\phi}_{j}^{a}\right\rangle=\delta_{i j} \\
\sum_{i}\left|\tilde{\phi}_{j}^{a}\right\rangle\left\langle\tilde{P}_{i}^{a}\right| .
\end{gathered}
$$

Assim obtemos os coeficientes $P_{n i}^{a}=\left\langle\tilde{P}_{i}^{a} \mid \tilde{\phi}_{j}^{a}\right\rangle$, substituindo tudo na equação (2.73), ficamos com a função de onda de Kohn-Sham all electron seguinte:

$$
\left|\psi_{n}\right\rangle=\left|\tilde{\psi}_{n}\right\rangle+\sum_{a} \sum_{i}\left(\left|\phi_{i}^{a}\right\rangle-\left|\tilde{\phi}_{i}^{a}\right\rangle\right)\left\langle\tilde{P}_{i}^{a} \mid \tilde{\psi}_{n}\right\rangle
$$

Para o método do PAW tornar-se prático, temos que levar em conta a aproximação de núcleo congelado. Na aproximação de frozen core, assume-se que os estados de caroço estão naturalmente localizados dentro das esferas aumentadas, além do que estados de caroço de átomos isolados não mudam pela formação de sólidos e moléculas. Então podemos resolver a equação de Kohn-Sham no método do PAW, apenas para os elétrons de valência e então somar a contribuição dos elétrons de caroço que pertencem a estados atômicos idênticos aos dos átomos isolados. Consequentemente para calcular o valor esperado de uma operador $\hat{O}$, no método PAW dentro da aproximação de frozen core obtemos:

$$
\langle\hat{O}\rangle=\sum_{n}^{\text {val }} f_{n}\left\langle\psi_{n}|\hat{O}| \psi_{n}\right\rangle+\sum_{a} \sum_{\alpha}^{\text {core }}\left\langle\phi_{\alpha}^{a, \text { core }}|\hat{O}| \phi_{\alpha}^{a, \text { core }}\right\rangle,
$$

onde $f_{n}$ representa a ocupação, e os índices $a, \alpha$ representam respectivamente os átomos e os estados atômicos destes. 


\subsection{Mecânica Quântica Relativística}

Em física do estado sólido, estrutura de bandas são feitas a partir da equação de Schrödinger. Neste contexto mostraremos que a interação spin órbita (SOC - Spin Orbit Coupling) pode ser incluída como uma correção relativística da equação de Schrödinger. SOC é um fenômeno conhecido que se manifesta em abrir a degenerescência de níveis energéticos em cálculos de estrutura eletrônica. Para tanto é necessário olhar as origens desta correção, seguindo a proposta de Dirac.

A equação de Dirac é a equação da mecânica quântica relativística para partículas de spin $\frac{1}{2}$. Ela é importante no trato de isolantes topológicos em dois aspectos. Primeiramente isolantes topológicos possuem um acoplamento spin órbita significativo que é consequência da equação de Dirac, cujo resultado permite transições topológicas via cruzamento de banda que serão analisados posteriormente. Além disso os hamiltonianos efetivos têm a estrutura matématica muito similar à equação de Dirac.

\subsubsection{Equação de Dirac}

Ao quantizar a equação não relativística da energia de uma partícula livre $\frac{\vec{p}^{2}}{2 m}=E$, obtemos a equação de Schrödinger do mesmo sistema. Agora se queremos um análogo à equação de Schrödinger porém relativística, temos que aplicar o princípio de correspondência quântica na equação (2.82) de energia momento dada pela mecânica da relatividade especial.

$$
\sqrt{(-i \hbar \nabla)^{2} c^{2}+m^{2} c^{4}} \Psi=i \hbar \frac{\partial}{\partial t} \Psi
$$

Klein e Gordon usaram o quadrado da equação (2.82) vista na equação (2.83), para tentar descrever partículas relativísticas, porém a função proposta por eles apresentava alguns problemas.

$$
\left(\nabla^{2}-\frac{1}{c^{2}} \frac{\partial^{2}}{\partial t^{2}}\right) \Psi-\frac{m^{2} c^{2}}{\hbar^{2}} \Psi=0
$$

A densidade de probabilidade dada pela equação (2.84) poderia não ser positivamente definida pela eventual escolha de $\Psi$ e $\frac{\partial \Psi}{\partial t}$. Além disso a dependência no tempo é não 
linear (o que impede a aplicação do princípio de superposição).

$$
\rho \sim \Psi^{*} \frac{\partial \Psi}{\partial t}-\frac{\partial \Psi^{*}}{\partial t} \Psi
$$

Apesar de descrever bem partículas sem spin a solução para problemas que descrevessem partículas elementares de spin $\frac{1}{2}$ era a equação proposta por Paul Dirac em 1928 [61], que nada mais é que a linearização da equação de Klein-Gordon:

$$
\left[\beta m c^{2}+c \vec{\alpha} \cdot \vec{p}\right] \Psi=i \hbar \frac{\partial \Psi}{\partial t}
$$

onde $\alpha_{i}$ e $\beta$ são matrizes 4 por 4 , dadas por:

$$
\alpha_{i}=\left[\begin{array}{cc}
0 & \sigma_{i} \\
\sigma_{i} & 0
\end{array}\right] ; \beta=\left[\begin{array}{cc}
I & 0 \\
0 & -I
\end{array}\right],
$$

onde $\sigma_{i}$ são as matrizes de Pauli. É importante notar aqui que a equação de Dirac obedece aos 6 postulados para a construção de uma equação relativística:

- A teoria é formulada em termos de um campo $\Psi$, com a interpretação estatística comum.

- A descrição dos fenômenos físicos na teoria é baseada em uma equação de movimento que descreve a evolução no tempo da amplitude do campo $\Psi$.

- A equação deve ser linear, para que seja valido o princípio da superposição.

- A equação deve ser relativisticamente invariante ou covariante.

- A densidade de probabilidade $\rho$ deve ser definida positivamente além de ser um invariante relativístico.

- A teoria deve ser consistente com o princípio da correspondência e no limite não relativístico deve reduzir-se à mecânica quântica não relativística.

\subsubsection{Acoplamento Spin Órbita}

Estamos interessados em incluir os efeitos relativísticos (como por exemplo acoplamento spin órbita) aos estados não relativísticos. Considerando o biespinor $\Psi_{T}=\left(\psi_{a}, \psi_{b}\right)$, 
que obedece à equação de Dirac, temos então o seguinte par de equações acopladas:

$$
\begin{aligned}
& \left(m c^{2}+V(r)\right) \psi_{a}+c(\vec{\sigma} \cdot \hat{p}) \psi_{b}=E \psi_{a}, \\
& c(\vec{\sigma} \cdot \hat{p}) \psi_{a}-\left(m c^{2}-V(r)\right) \psi_{b}=E \psi_{b} .
\end{aligned}
$$

Substituindo uma equação na outra obtemos a seguinte relação dos biespinores:

$$
\begin{aligned}
& \psi_{b}=\frac{1}{2 m c^{2}+E^{\prime}-V(r)} c(\vec{\sigma} \cdot \hat{p}) \psi_{a}, \\
& c(\vec{\sigma} \cdot \hat{p}) \psi_{b}=\left(E^{\prime}-V(r)\right) \psi_{a} .
\end{aligned}
$$

Definimos um shift de energia sobre a energia de repouso $E^{\prime}=E-m c^{2}$. No limite não relativístico $E^{\prime}<<m c^{2}$, temos que o $\psi_{b}$ é muito menor que $\psi_{a}$, assim podemos compactar a equação acima da seguinte forma:

$$
c(\vec{\sigma} \cdot \hat{p}) \frac{1}{2 m c^{2}+E^{\prime}-V(r)} c(\vec{\sigma} \cdot \hat{p}) \psi_{a}=\left(E^{\prime}-V(r)\right) \psi_{a}
$$

Neste limite podemos fazer uma expansão em torno de $v / c$, obtendo:

$$
\begin{aligned}
\frac{1}{2 m c^{2}+E^{\prime}-V(r)} & =\frac{1}{2 m c^{2}}\left\{\frac{1}{1+\frac{E^{\prime}-V(r)}{2 m c^{2}}}\right\}, \\
& =\frac{1}{2 m c^{2}}\left\{1-\frac{E^{\prime}-V(r)}{2 m c^{2}}+\ldots\right\} .
\end{aligned}
$$

Se considerarmos termos até primeira ordem ficamos com:

$$
c(\vec{\sigma} \cdot \hat{p}) \frac{1}{2 m c^{2}}\left(1-\frac{E^{\prime}-V(r)}{2 m c^{2}}\right) c(\vec{\sigma} \cdot \hat{p}) \psi_{a}=\left(E^{\prime}-V(r)\right) \psi_{a} .
$$

Note que, em ordem zero, recuperamos a equação de Schödinger não relativística dada pela equação (2.92).

$$
\frac{\hat{p}^{2}}{2 m}=E^{\prime}-V \rightarrow E^{\prime}=\frac{\hat{p}^{2}}{2 m}+V
$$

Agora olhando apenas o termo de segunda ordem e usando que $E^{\prime}-V \approx \frac{\hat{p}^{2}}{2 m}$, obtemos a seguinte hamiltoniana:

$$
\hat{H} \simeq \frac{\hat{p}^{2}}{2 m}+V-\frac{\hat{p}^{4}}{8 m^{3} c^{2}}+\frac{1}{2 m^{2} c^{2}} \vec{S} \cdot(\nabla V \times \hat{p})+\frac{\hbar}{2 m^{2} c^{2}} \nabla^{2} V,
$$

com $\vec{S}=\frac{\hbar \vec{\sigma}}{2}$. Notamos três termos de correções relativísticas. O terceiro termo da hamiltoniana é apenas uma correção da massa com a velocidade. O quarto termo é chamado de correção spin órbita e surge quando mudamos nosso sistema de referência, com o núcleo orbitando o elétron e não o contrário. Este movimento nuclear circular gera 
uma corrente que consequentemente gera um campo magnético, este por sua vez acopla com o momento de spin do elétron criando uma quebra de degenerescência dependendo da orientação do spin. Por último temos o termo de Darwin que muda o potencial efetivo do núcleo, este termo pode ser interpretado como uma suavização da interação entre o elétron e o núcleo, que apresentam rápidas oscilações no espaço.

Nos programas usuais baseados em DFT como VASP e SIESTA, para se incluir efeitos de spin órbita temos que resolver de maneira diferente, pois não temos o potencial, logo teremos que construí-lo de maneira análoga ao capítulo 2, onde tínhamos a seguinte equação:

$$
V_{p s}^{l} \propto \sum_{i l m}\left|Y_{l m}\right\rangle \tilde{V}_{p s}^{l}\left(\left|\vec{r}-\vec{R}_{i}\right|\right)\left\langle Y_{l m}\right|
$$

Neste sentido para haver um acoplamento do spin com o momento angular orbital, é necessário acoplar ambos quanticamente de forma que temos $j_{1}=l, j_{2}=s=\frac{1}{2}$ com $m_{1}=m_{l}$ e $m_{2}=m_{s}= \pm \frac{1}{2}$. É simples notar que na base de momento angular total temos $j=l \pm \frac{1}{2}$, com $l>0$. Logo para cada valor de $l, j$ assume dois possíveis valores. Com isso ficamos com o pseudopotencial reformulado:

$$
V_{p s}^{l} \propto \sum_{i l m}\left|\chi_{m}^{j=l+\frac{1}{2}}\right\rangle \tilde{V}_{p s}^{l+\frac{1}{2}}\left(\left|\vec{r}-\vec{R}_{i}\right|\right)\left\langle\chi_{m}^{j=l+\frac{1}{2}}|+| \chi_{m^{\prime}}^{j=l-\frac{1}{2}}\right\rangle \tilde{V}_{p s}^{l-\frac{1}{2}}\left(\left|\vec{r}-\vec{R}_{i}\right|\right)\left\langle\chi_{m^{\prime}}^{j=l-\frac{1}{2}}\right|
$$

Esta última equação está na base de momento angular total. Para fins práticos temos que voltar para nossa base de harmônicos esféricos com spin na qual foi elaborada a teoria do pseudopotencial. Para tanto necessitamos dos coeficientes de Clebsch-Gordan. Logo ao aplicar a completeza da base dos harmônicos esféricos obtemos a equação (2.96), na qual foi feita apenas a parte de $j=l+\frac{1}{2}$.

$$
V_{p s}^{l}\left(l+\frac{1}{2}\right) \propto \sum_{i l m} \sum_{l^{\prime} m^{\prime}} \sum_{l^{\prime \prime} m^{\prime \prime}}\left\langle l^{\prime}, m^{\prime} \mid \chi_{m}^{j=l+\frac{1}{2}}\right\rangle\left|l^{\prime}, m^{\prime}\right\rangle \tilde{V}_{p s}^{l+\frac{1}{2}}\left(\left|\vec{r}-\vec{R}_{i}\right|\right)\left\langle l^{\prime \prime}, m^{\prime \prime}\right|\left\langle\chi_{m}^{j=l+\frac{1}{2}} \mid l^{\prime \prime}, m^{\prime \prime}\right\rangle
$$

Note que os estados $\left|\chi_{m}^{j}\right\rangle$, também são escritos da forma $\left|j_{1} j_{2}, j m\right\rangle$, e agora $|l, m\rangle$ a qual inclui spin pode ser escrito como $\left|l, m_{l}\right\rangle \otimes\left|s, m_{s}\right\rangle=\left|j_{1} j_{2}, m_{l} m_{s}\right\rangle$. Pelo fato de ambas bases serem autovalores de $J_{1}$ e $J_{2}$ vamos omitir o índices $j_{1}$ e $j_{2}$. Note que $m_{l}=m \pm \frac{1}{2}$ e 
$m_{s}=\frac{1}{2}$. A matriz de transformação de uma base para outra é dada pela equação (2.97).

$$
\left(\begin{array}{c}
\left|\chi_{m}^{j=l+\frac{1}{2}}\right\rangle \\
\left|\chi_{m}^{j=l-\frac{1}{2}}\right\rangle
\end{array}\right)=\left(\begin{array}{cc}
\sqrt{\frac{l+m+\frac{1}{2}}{2 l+1}} & \sqrt{\frac{l-m+\frac{1}{2}}{2 l+1}} \\
-\sqrt{\frac{l-m+\frac{1}{2}}{2 l+1}} & \sqrt{\frac{l+m+\frac{1}{2}}{2 l+1}}
\end{array}\right)\left(\begin{array}{l}
\left|m_{l}=m+\frac{1}{2}, m_{s}=\frac{1}{2}\right\rangle \\
\left|m_{l}=m-\frac{1}{2}, m_{s}=\frac{1}{2}\right\rangle
\end{array}\right) .
$$

Ao substituir na equação (2.96), ficamos com:

$$
\begin{gathered}
V_{p s}^{l} \propto \quad \sum_{i l m}\left|m_{l}, m_{s}\right\rangle\left(\frac{l+m+\frac{1}{2}}{2 l+1}+\frac{l-m+\frac{1}{2}}{2 l+1}\right) \tilde{V}_{p s}^{l+\frac{1}{2}}\left(\left|\vec{r}-\vec{R}_{i}\right|\right)\left\langle m_{l}, m_{s}\right|, \\
+\sum_{i l m}\left|m_{l}, m_{s}\right\rangle\left(\frac{l-m+\frac{1}{2}}{2 l+1}+\frac{l+m+\frac{1}{2}}{2 l+1}\right) \tilde{V}_{p s}^{l-\frac{1}{2}}\left(\left|\vec{r}-\vec{R}_{i}\right|\right)\left\langle m_{l}, m_{s}\right|, \\
=\quad \sum_{i l m} \frac{2 l+1}{2 l+1} \tilde{V}_{p s}^{l+\frac{1}{2}}\left(\left|\vec{r}-\vec{R}_{i}\right|\right)\left|m_{l}, m_{s}\right\rangle\left\langle m_{l}, m_{s}\left|+\frac{2 l+1}{2 l+1} \tilde{V}_{p s}^{l-\frac{1}{2}}\left(\left|\vec{r}-\vec{R}_{i}\right|\right)\right| m_{l}, m_{s}\right\rangle\left\langle m_{l}, m_{s}\right| .
\end{gathered}
$$

Usando o fato que na base $\left|j_{1} j_{2}, m_{l} m_{s}\right\rangle J^{2}, L^{2}$ e $S^{2}$ são observáveis, temos que $\vec{L} \cdot \vec{S}=$ $J^{2}-L^{2}-S^{2}$ também o é desta forma podemos escrever:

$$
\begin{array}{lll}
(2 l+1)\left|m_{l}=m+\frac{1}{2}, \frac{1}{2}\right\rangle=(l+1+2 \vec{L} \cdot \vec{S}) & \left|m_{l}=m+\frac{1}{2}, \frac{1}{2}\right\rangle \\
(2 l+1)\left|m_{l}=m-\frac{1}{2}, \frac{1}{2}\right\rangle=(l-2 \vec{L} \cdot \vec{S}) & \left|m_{l}=m-\frac{1}{2}, \frac{1}{2}\right\rangle .
\end{array}
$$

E assim ficamos com o seguinte pseudopotencial:

$$
V_{p s}^{i o n}=V_{s c}+V_{S O}=\sum_{i l m}\left[V_{s c}^{l}\left(\left|\vec{r}-\vec{R}_{i}\right|\right) I_{\sigma}+V_{S O}^{l}\left(\left|\vec{r}-\vec{R}_{i}\right|\right)\right]|l, m\rangle\langle l, m|
$$

sendo $I_{\sigma}$ o operador identidade no espaço de spin, e dadas as equações (2.101) e (2.102), o hamiltoniano toma o formato da equação (2.103).

$$
\begin{gathered}
V_{S O}^{l}(r)=\frac{2}{2 l+1}\left[V_{p s}^{j=l+\frac{1}{2}}-V_{p s}^{j=l-\frac{1}{2}}\right], \\
V_{s c}^{l}(r)=\frac{1}{2 l+1}\left[(l+1) V_{p s}^{j=l+\frac{1}{2}}+l V_{p s}^{j=l-\frac{1}{2}}\right], \\
\hat{H}=\hat{T}+\hat{V}^{s c}+\hat{V}^{S O}+\hat{V}^{H}+\hat{V}^{x c},
\end{gathered}
$$

onde temos repectivamente os termos de energia cinética, escalar relativístico, interação spin órbita, de Hatree e o potencial de troca e correlação. 



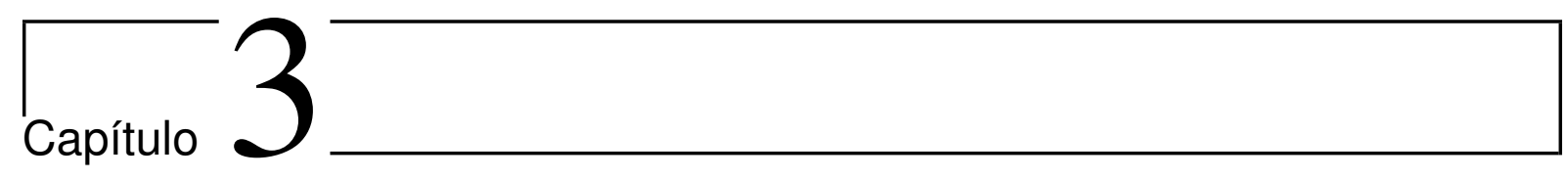

\section{Fundamentos Téoricos}

Neste capítulo apresentamos os conceitos teóricos necessários para o entendimento dos isolantes topológicos, tanto suas propriedades intrínsicas quanto as ferramentas úteis para a abordagem do trabalho.

\subsection{Topologia de Bandas e suas ferramentas}

Topologia é um ramo antigo na matemática. Pelo teorema de Gauss-Bonnet existe uma relação entre a integral de superfície da curvatura gaussiana $K$, feita sobre a superfície geometrica de um objeto $M$, e o chamado $g$ de genus conhecido como número de enrolamento, dada pela equação (3.1) que classifica as superfícies por sua topologia. Desta forma como visto na figura 3 uma rosca e uma esfera possuem $g=1$ e $g=0$ e não existe uma transformação que leve de uma até a outra sem que se rompa a superfície.

$$
\int_{M} K d A=2-2 g
$$

Analogamente nos TIs suas funções de onda descrevendo os estados eletrônicos que geram o espaço de Hilbert tem uma topologia não trivial também descrita por um invariante. Esta última característica é um atributo de sistemas com gap, e para que a topologia mude, o gap tem que fechar. Uma importante consequência de uma topologia não trivial associada às funções de onda de um isolante é que estados metálicos de interface necessariamente irão surgir. 


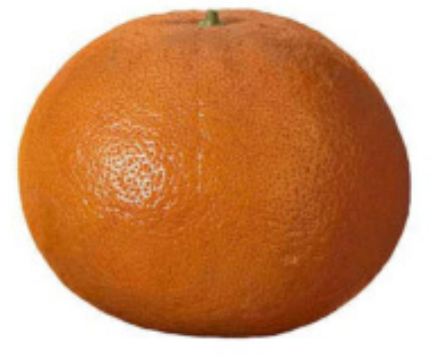

(a) Esfera com genus $=0$.

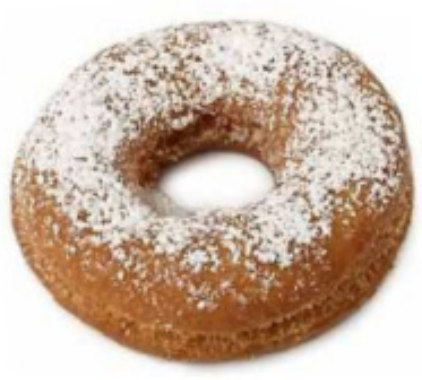

(b) Rosca com genus $=1$.

Figura 3: (a)-(b) Representam duas superfícies que diferem topologicamente por seu genus, sendo 0 para a esfera e 1 para a rosca. Imagem da referência [62].

Nesta tese e neste capítulo o interesse é voltado para os isolantes topológicos do tipo $Z_{2}$, ou seja descritos por um invariante chamado $Z_{2}$, cuja topologia não trivial advém da forte interação spin órbita. Tal inversão de bandas no material devido a interação spin órbita não existe no vácuo, logo um estado metálico caracterizando a inversão deve ocorrer na superfície ou borda deste material. Além disso nestes materiais na borda ou superfície a TRS ( Time Reversal Symmetry) proporciona uma textura única de spin vista na figura 4, na qual a física relativística de férmions de Dirac torna-se importante. Para
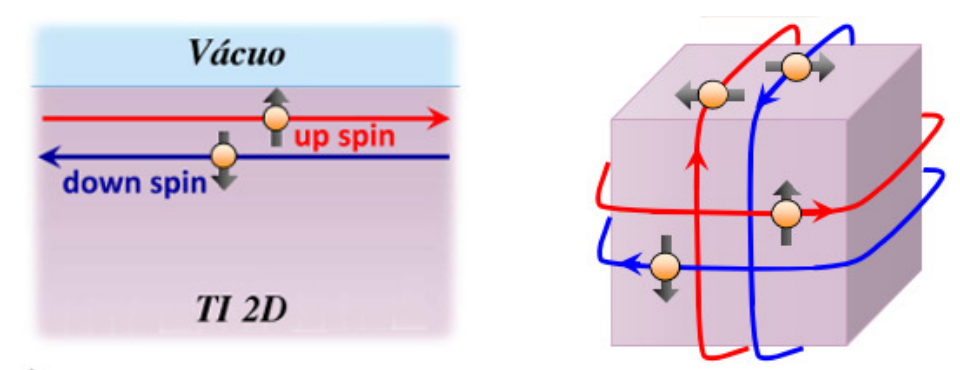

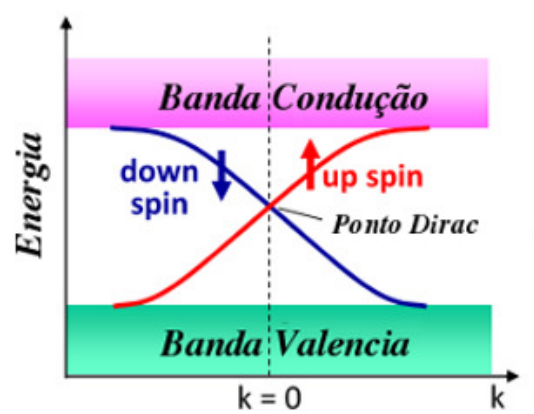

(a) QSHE em $2 D$.

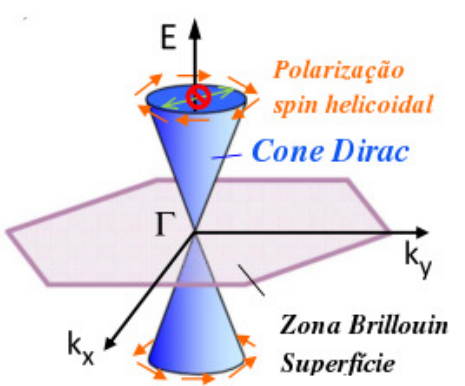

(b) QSHE em 3D.

Figura 4: (a) Representação esquemática do espaço real (painel acima) e do espaço recíproco (painel abaixo) de (a) um isolante topológico em $2 D$ e (b) um isolante topológico em 3D. Imagem da referência [63]. 
o entendimento desta nova área topológica $Z_{2}$ e seus invariantes é importante estudar o papel da simetria de reversão temporal para partículas de spin $\frac{1}{2}$.

\subsubsection{Operador reversão temporal e Teorema de Kramer}

A aplicação do operador do TRS é uma operação que representa mais especificamente inversão de movimento. Classicamente uma partícula sujeita a uma força que faça com que ela caminhe para direita, ao reverter o movimento ou seja $t^{\prime} \rightarrow-t^{\prime}$ temos que a mesma partícula se moverá para a esquerda ou seja $\left.\vec{p}\right|_{t=t^{\prime}} \rightarrow-\left.\vec{p}\right|_{t=-t^{\prime}}$ sendo $\vec{p}$ momento linear da partícula.

Quanticamente para partículas de spin $\frac{1}{2}$ temos que o operador Time-Reversal, $\Theta$ é antiunitário e representado por:

$$
\Theta=e^{-i \frac{\pi S_{y}}{\hbar}} K=-i \sigma_{y} K
$$

Aqui $K$ é um operador que aplicado em um número complexo retorna o complexo conjugado, e $S_{y}$ o operador momento angular de spin na direção $\hat{y}$ e $\sigma_{y}$ é a matriz de Pauli na direção $\hat{y}$. Nesta simetria alguns operadores podem ser par ou ímpar. Aqui analisaremos dois deles, o operador de momento e o de spin. O momento é impar sob reversão temporal como descrito na seguinte equação:

$$
\begin{aligned}
\Theta \vec{p}\left(\begin{array}{c}
\Psi_{1} \\
\Psi_{2}
\end{array}\right) \Theta^{-1} & =i \sigma_{y} K \vec{p}\left(\begin{array}{l}
\Psi_{1} \\
\Psi_{2}
\end{array}\right) i \sigma_{y}^{-1} K^{-1}, \\
& =\left(\begin{array}{cc}
0 & 1 \\
-1 & 0
\end{array}\right)\left(\begin{array}{cc}
-\frac{\hbar}{i} \nabla \Psi_{1} & 0 \\
0 & \frac{\hbar}{i} \nabla \Psi_{2}
\end{array}\right)\left(\begin{array}{cc}
0 & -1 \\
1 & 0
\end{array}\right), \\
& =\left(\begin{array}{cc}
-\frac{\hbar}{i} \nabla \Psi_{1} & 0 \\
0 & -\frac{\hbar}{i} \nabla \Psi_{2}
\end{array}\right)=-\vec{p}\left(\begin{array}{c}
\Psi_{1} \\
\Psi_{2}
\end{array}\right) .
\end{aligned}
$$

Para o spin vamos olhar apenas o caso de $S_{x}$, pois o tratamento é análogo nas outras direções. E assim como visto na equação (3.4) o spin é ímpar sob esta simetria.

$$
\Theta S_{x} \Theta^{-1}=\frac{\hbar}{2}(-i) \sigma_{y} K \sigma_{x}(-i) \sigma_{y}^{-1} K^{-1}=\frac{\hbar}{2}(-i)^{2} \sigma_{y} \sigma_{x} \sigma_{y}^{-1 *}=-\frac{\hbar}{2} \sigma_{x}=-S_{x} .
$$

Desta forma a correção devido a interação spin órbita $\frac{1}{2 m^{2} c^{2}} \vec{S} \cdot(\nabla V \times \hat{p})$, que possui dois operadores cujos autovalores são ímpares sob reversão temporal, é invariante sob 
esta simetria. Sabendo disso vamos olhar qual vínculo criamos em uma hamiltoniana periódica (com spin órbita) invariante sobre simetria de reversão temporal. Vimos que em um sistema periódico podemos reescrever a equação energética de autovalores da seguinte forma:

$$
H\left|\phi_{n \vec{k}}\right\rangle=e_{n \vec{k}}\left|\phi_{n \vec{k}}\right\rangle
$$

no qual pelo teorema de Bloch temos:

$$
\left|\phi_{n \vec{k}}\right\rangle=e^{i \vec{k} \cdot \vec{r}}\left|u_{n \vec{k}}\right\rangle
$$

onde $\left|u_{n \vec{k}}\right\rangle$ são autoestados da hamiltoniana de Bloch (Hamiltoniana no espaço de momentos), vista na equação (3.7).

$$
H(\vec{k})=e^{-i \vec{k} \cdot \vec{r}} H e^{i \vec{k} \cdot \vec{r}}
$$

Agora se $[\Theta, H]=0$, o que não é verdade se $H$ contém termos magnéticos, $H(\vec{k})$ satisfaz a seguinte condição:

$$
H(-\vec{k})=\Theta H(\vec{k}) \Theta^{-1}
$$

Esta identidade significa que as bandas de um sistema que obedeça a TRS vem em pares, chamados de pares de Kramer. Esta simetria também é responsável pela ausência de retroespalhamento nestes materiais como visto no apêndice E. Um elemento importante é a existência de pontos especiais $\vec{k}=\Gamma_{i}$ na borda da zona de Brillouin onde $-\Gamma_{i}=\Gamma_{i}+\vec{G}$, sendo $\vec{G}$ um vetor da rede recíproca. Nestes pontos chamados de TRIM ( Time Reversal Invariant Momenta), os autoestados são Kramer degenerados como visto na figura 5. Existem duas maneiras possíveis de conectar na estrutura de banda os TRIMs, nos quais a degenerescência de Kramer ocorre. O primeiro chamado de STI (Strong Topological Insulator), e o outro de WTI (Weak Topological Insulator). Isso porque no WTI podemos dopá-lo de maneira que seu nível de Fermi fique em uma região sem estados, o que corresponderia a um isolante trivial, o que não seria possível no caso do STW.

É a mudança na polarização de "reversão temporal", definida por Kane e Mele [5] e descrita na forma de um invariante $Z_{2}$ em seções posteriores, entre cada TRIM da zona de Brillouin que determina de que maneira os estados degenerados de Kramer se conectam. 


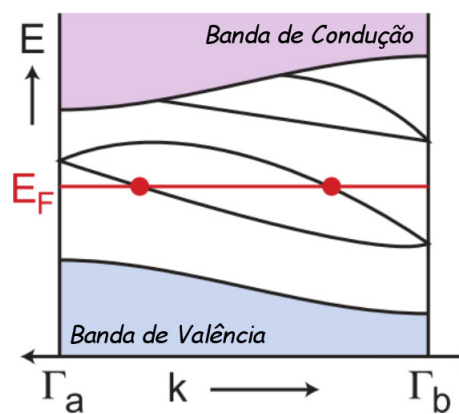

(a) Isolante trivial.

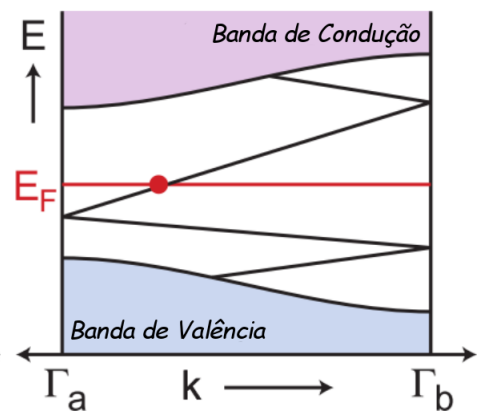

(b) Isolante topológico.

Figura 5: Degenerescência de Kramer, onde ambas as figuras possuem uma dupla degenerescência em $\Gamma_{a}$ e $\Gamma_{b}$. Na figura da esquerda a banda intercepta um número par de vezes o nivel de Fermi formando um WTI (Weak Topological Insulator). Na figura da direita a banda intercepta um número ímpar de vezes o nível de Fermi formando um STI (Strong Topological Insulator). Imagem da referência [62].

\subsection{Invariantes topológicos e fases geométricas em teoria de ban- das}

Até a metade dos anos 80 a fase quântica acumulada no processo de evolução adiabática era geralmente ignorada, inclusive Fock [64] apresentou um argumento dizendo que a fase poderia ser considerada como a própria unidade. Porém, este resultado foi derivado pela hipótese de evoluções não cíclicas. Foi apenas em 1984 que Michael Berry [65] mostrou a importância em sistemas quânticos de se considerar a fase durante um ciclo fechado adiabático, de natureza puramente geométrica cuja consequências aparecem nas propriedades eletrônicas e na classificação topológica do material.

Aqui iremos introduzir alguns conceitos de polarização que estão intimamente relacionados com a fase de Berry das autofunções de Bloch enquanto esta última atravessa a zona de Brillouin. Veremos também como relacionar esta fase com os invariantes topológicos conhecidos hoje em dia.

\subsubsection{Fase de Berry}

Vamos escrever uma hamiltoniana de um sistema definido pelo parâmetro $\vec{R}(t)$ (com $\vec{R}(t)$ um vetor no espaço de parâmetro que depende do tempo), da forma $H[\vec{R}(t)]$ cujo n-ésimo autoestado é dado por $|n, \vec{R}(t)\rangle$. Assim a equação de Schrödinger toma a seguinte 
forma:

$$
H[\vec{R}(t)]|n, \vec{R}(t)\rangle=E_{n}(\vec{R}(t))|n, \vec{R}(t)\rangle
$$

Agora vamos supor que $\vec{R}(t)$ mude adiabaticamente a partir de $t=0$ cujo valor seria $R_{0}$. A equação de Schrödinger ficaria como:

$$
H[\vec{R}(t)]|n, \vec{R}(t)\rangle=i \hbar \frac{\partial}{\partial t}|n, \vec{R}(t)\rangle .
$$

Pelo teorema adiabático teremos num tempo $t$ o seguinte autoestado:

$$
|n, \vec{R}(t)\rangle=e^{\left(\frac{i}{\hbar} \int_{0}^{t} d t^{\prime} L_{n}\left(\vec{R}\left(t^{\prime}\right)\right)\right)}|n, \vec{R}(t)\rangle
$$

onde $L_{n}\left(\vec{R}\left(t^{\prime}\right)\right)=i \hbar \dot{\vec{R}}(t) \cdot\left\langle n, \vec{R}(t)\left|\nabla_{R}\right| n, \vec{R}(t)\right\rangle-E_{n}[\vec{R}(t)]$. Substituindo isso na equação (3.11) obtemos a seguinte equação:

$$
|n, \vec{R}(t)\rangle=e^{-\int_{0}^{t} d t^{\prime} \dot{\vec{R}}\left(t^{\prime}\right) \cdot\left\langle n, \vec{R}(t)\left|\nabla_{R}\right| n, \vec{R}(t)\right\rangle} e^{\frac{i}{\hbar} \int_{0}^{t} d t^{\prime} E_{n}\left[\vec{R}\left(t^{\prime}\right)\right]}|n, \vec{R}(t)\rangle .
$$

As duas fases podem ser separadas, a primeira chamada de dinâmica, e a segunda de geométrica. Esta última é o efeito quântico do acúmulo da fase durante a evolução adiabática. Caso a evolução seja cíclica ou seja para $t=T \operatorname{com} \vec{R}(T)=R_{0}$, a fase chama-se fase de Berry e escreve-se da seguinte forma:

$$
\begin{aligned}
\gamma_{n}[C] & =\int_{0}^{T} i d t \dot{\vec{R}}(t) \cdot\left\langle n, \vec{R}(t)\left|\nabla_{R}\right| n, \vec{R}(t)\right\rangle, \\
& =\oint_{C} i d \vec{R} \cdot\left\langle n, \vec{R}\left|\nabla_{R}\right| n, \vec{R}\right\rangle, \\
& =-\oint_{C} d \vec{R} \cdot \vec{A}_{n}(\vec{R}) .
\end{aligned}
$$

Onde $\vec{A}_{n}(\vec{R})=-i\left\langle n, \vec{R}\left|\nabla_{R}\right| n, \vec{R}\right\rangle$, chamado de conexão de Berry em analogia ao eletromagnetismo. Usando o teorema de Stokes podemos reescrevê-la:

$$
\gamma_{n}=-\int_{S} \nabla_{R} \times \vec{A}_{n}(\vec{R}) \cdot d \vec{S}=-\int_{S} B_{n}(\vec{R}) \cdot d \vec{S}
$$

$B_{n}(\vec{R})$ é chamado de curvatura de Berry. É importante notar que a conexão de Berry é uma quantidade dependente de calibre, ou seja, dada uma transformação como:

$$
\begin{array}{lc}
|n, \vec{R}\rangle= & e^{i \zeta(\vec{R})}|n, \vec{R}\rangle, \\
A_{n}^{\prime}(\vec{R}) & =A_{n}(\vec{R})-\frac{\partial}{\partial \vec{R}} \zeta(\vec{R}) .
\end{array}
$$

Uma possível escolha de $\zeta(\vec{R})$ faria com que o termo $A_{n}^{\prime}(\vec{R})$ se anulasse. Agora para evoluções cíclicas, onde $\vec{R}(0)=\vec{R}(T)$ a equação (3.15) tem um valor bem definido. Assim $\zeta(\vec{R}(0))-\zeta\left(\vec{R}\left(t_{f}\right)\right)=2 \pi m$ com $m$ sendo um inteiro. Desta forma a conexão de Berry pode ser considerada um invariante topológico sob qualquer calibre, o que veremos a seguir. 


\subsubsection{TKNN e número de Chern}

O invariante topológico definido no QHE, o invariante TKNN (nome dedicados aos pesquisadores - D. J. Thouless, M. Kohmoto, P. Nightingale e M. den Nijs) [2], está intimamente relacionado à fase de Berry. Aqui iremos derivar este número em um sistema eletrônico $2 \mathrm{D}$ de tamanho $L \mathrm{x} L$ sob a atuação de um campo magnético perpendicular ao plano. Os campos elétrico $\vec{E}$ e magnético $\vec{B}$, estão aplicados nas direções $\hat{y}$ e $\hat{z}$ respectivamente, como visto na figura 6 .

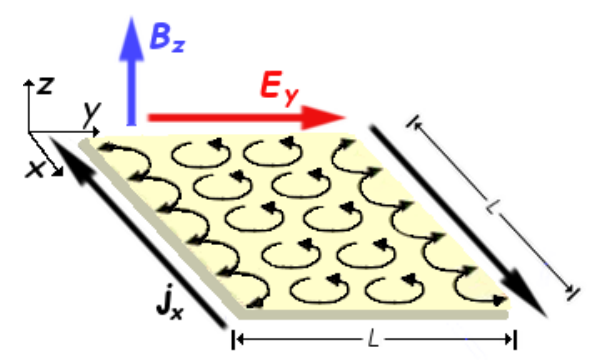

Figura 6: Efeito Hall quântico. O campo magnético $\vec{B}$ na direção $\hat{z}$ provoca órbitas ciclotrônicas nos elétrons. A quebra destas órbitas na borda gera uma densidade de corrente elétrica $\vec{j} n a$ direção $\hat{x}$ além de um campo elétrico $\vec{E}$ na direção $\hat{y}$.

Se tratarmos o efeito do campo elétrico como um potencial perturbativo $V=-e E y$, poderíamos usar a teoria de perturbação para aproximar o autoestado $|n\rangle_{E}$ por:

$$
|n\rangle_{E}=|n\rangle+\sum_{m \neq n} \frac{\langle m|-e E y| n\rangle}{E_{n}-E_{m}}|m\rangle+\ldots
$$

Em primeira ordem podemos usar o autoestado perturbado para calcular o valor esperado da densidade de corrente $j_{x}$ ao longo da direção $\hat{x}$ da seguinte maneira:

$$
\begin{aligned}
& \left\langle j_{x}\right\rangle_{E}=\sum_{n} f\left(E_{n}\right)\left\langle\left. n\right|_{E}\left(\frac{e v_{x}}{L^{2}}\right) \mid n\right\rangle_{E} \\
& =\left\langle j_{x}\right\rangle_{E=0}+\frac{1}{L^{2}} \sum_{n} f\left(e_{n}\right) \sum_{m \neq n} \quad\left(\frac{\left\langle n\left|e v_{x}\right| m\right\rangle\left\langle m\left|-e E_{y}\right| n\right\rangle}{E_{n}-E_{m}}\right. \\
& \left.+\frac{\left\langle n\left|-e E_{y}\right| m\right\rangle\left\langle m\left|e v_{x}\right| n\right\rangle}{E_{n}-E_{m}}\right),
\end{aligned}
$$

onde $\left\langle j_{x}\right\rangle_{E=0}=0, v_{x}$ é a velocidade do elétron na direção $\hat{x}$ e $f\left(E_{n}\right)$ a função de distribuição de Fermi-Dirac. Sabendo que a equação de movimento de Heisenberg é dada por $\frac{d y}{d t}=$ $v_{y}=\frac{1}{i \hbar}[y, H]$ podemos escrever o seguinte:

$$
\left\langle m\left|v_{y}\right| n\right\rangle=\frac{1}{i \hbar}\left(E_{n}-E_{m}\right)\langle m|y| n\rangle .
$$


Se substituimos a equação (3.18) na equação (3.17) obtemos:

$$
\sigma_{x y}=\frac{\left\langle j_{x}\right\rangle_{E}}{E}=\frac{-i \hbar e^{2}}{L^{2}} \sum_{n} \sum_{m \neq n} f\left(E_{n}\right) \frac{\left\langle n\left|v_{x}\right| m\right\rangle\left\langle m\left|v_{y}\right| n\right\rangle-\left\langle n\left|v_{y}\right| m\right\rangle\left\langle m\left|v_{x}\right| n\right\rangle}{\left(E_{n}-E_{m}\right)^{2}} .
$$

Ao considerar um sistema periódico no qual os autoestados são dados por funções de Bloch $\left|u_{n \vec{k}}\right\rangle$, ficamos com:

$$
\left\langle u_{m \vec{k}^{\prime}}\left|v_{\mu}\right| u_{n \vec{k}}\right\rangle=\frac{1}{i \hbar}\left(E_{n \vec{k}}-E_{m \vec{k}^{\prime}}\right)\left\langle u_{m \vec{k}^{\prime}}\left|\frac{\partial}{\partial k_{\mu}}\right| u_{n \vec{k}}\right\rangle .
$$

Substituindo a equação 3.20 na equação (3.19) obtemos:

$$
\sigma_{x y}=-\frac{i \hbar e^{2}}{L^{2}} \sum_{\vec{k}} \sum_{n} f\left(E_{n \vec{k}}\right)\left(\frac{\partial}{\partial k_{x}}\left\langle u_{n \vec{k}^{\prime}}\left|\frac{\partial}{\partial k_{y}}\right| u_{n \vec{k}}\right\rangle-\frac{\partial}{\partial k_{y}}\left\langle u_{n \vec{k}^{\prime}}\left|\frac{\partial}{\partial k_{x}}\right| u_{n \vec{k}}\right\rangle\right) .
$$

A conexão de Berry no espaço de momento é dada por $\vec{a}_{n}(\vec{k})=-i\left\langle u_{n \vec{k}^{\prime}}\left|\nabla_{k}\right| u_{n \vec{k}}\right\rangle$, com isso a condutividade Hall (A temperatura $T=0$, que implica $f\left(E_{n \vec{k}}\right)=1$ ) fica:

$$
\begin{aligned}
\sigma_{x y} & =\frac{\nu e^{2}}{h}, \\
\nu & =\sum_{n} \int_{B Z} \frac{d^{2} \vec{k}}{2 \pi} \vec{a}_{n}(\vec{k}) .
\end{aligned}
$$

Note que $\nu=\sum_{n} \nu_{n}$ onde $\nu_{n}=\int_{B Z} \frac{d^{2} \vec{k}}{2 \pi} \vec{a}_{n}(\vec{k})$ de forma que obtemos:

$$
\nu_{n}=-\frac{1}{2 \pi} \gamma_{n}[\partial B Z]
$$

onde $\gamma_{n}[\partial B Z]$ significa que a fase de Berry foi integrada no caminho fechado correspondente a zona de Brillouin. Esta fase é integrada em todas as bandas ocupadas da primeira zona de Brillouin, e corresponde a um invariante que descreve um isolante topológico com quebra de TRS, também chamado de número de Chern que proporciona o QHE.

\subsubsection{Invariante $Z_{2}$ e polarização de reversão temporal}

Existem várias formulações diferentes para o invariante $Z_{2}$. Para cada caso, dependendo das simetrias, o cálculo toma formatos distintos. Temos Kane e Mele 2005 [5], Fu e Kane 2006-2007 [66,67], Fukui e Hatsugai 2007 [68], Moore e Balents em 2007 [13], Fukui, Fujiwara e Hatsugai em 2008 [69], Qi, Hughes e Zhang 2008 [70], Roy em 2009 [14] e Wang, Qi e Zhang em 2010 [71]. Aqui falaremos da teoria da fase de Berry para a polarização, elaborada nos anos 90. Seguido de importante contribuição preliminar por 
Resta [72], o principal desenvolvimento foi introduzido por King-Smith e Vanderbilt [73], e subsequentemente revisado pelo próprio Resta [74].

Como vimos anteriormente em um material onde existe simetria de reversão temporal o teorema de Kramer garante que os estados venham em, no mínimo, pares degenerados. Assumindo que não existe degenerescência a menos daquela devida à simetria de reversão temporal, temos que $2 N$ autoestados podem ser divididos em $N$ pares. Ao aplicar o operador de reversão temporal em um autoestado revertido obtemos o autoestado original com um fator de fase $\chi_{k n}$ :

$$
\begin{aligned}
\left|u_{n k}^{I}\right\rangle & =-e^{i \chi_{k n}} \Theta\left|u_{n k}^{I I}\right\rangle, \\
\left|u_{n k}^{I I}\right\rangle & =e^{i \chi_{-k n}} \Theta\left|u_{n k}^{I}\right\rangle,
\end{aligned}
$$

onde $n=1, \ldots, N$ é um índice de banda e $\Theta$ o operador de reversão temporal onde $\Theta^{2}=-1$. Aqui neste exemplo unidimensional a degenerescência ocorre em $k=0, \pi$. Define-se então, analogamente ao número de Chern, uma polarização parcial onde:

$$
P^{s}=\frac{1}{2 \pi} \int_{-\pi}^{\pi} d k A^{s}(k)
$$

$\operatorname{Com} s=I, I I$ e $A(k)=A^{I}+A^{I I}=-i\left\langle u_{n k}^{I}|\nabla| u_{n k}^{I}\right\rangle-i\left\langle u_{n k}^{I I}|\nabla| u_{n k}^{I I}\right\rangle=\sum_{n} \operatorname{tr}\left(a_{n n}(k)\right)$. Podemos assim obter a carga polarização de Resta como $P_{\rho}=P^{I}+P^{I I}$, porém estamos interessados aqui em obter o que definiremos de polarização de Time-Reversal proposta por Fu e Kane [66] visto na equação (3.26).

$$
P_{\Theta}=P^{I}-P^{I I}=2 P^{I}-P_{\rho} .
$$

Intuitivamente, $P_{\Theta}$ nos dá a diferença entre carga de polarização entre bandas spin up e spin down. Neste contexto uma matriz conveniente para nossos fins é a chamada matriz de reversão temporal dada por $\omega_{n m}(k)=\left\langle u_{n,-k}|\Theta| u_{m, k}\right\rangle$, que dado a (3.24), tem a seguinte forma matricial:

$$
\omega_{n m}(k)=\left(\begin{array}{lllll}
0 & e^{-i \chi_{1 k}} & 0 & 0 & \cdots \\
-e^{-i \chi_{1 k}} & 0 & 0 & 0 & \ldots \\
0 & 0 & 0 & e^{-i \chi_{2 k}} & \ldots \\
0 & 0 & -e^{-i \chi_{2 k}} & 0 & \cdots \\
\vdots & \vdots & \vdots & \vdots & \ddots
\end{array}\right)
$$


Note que se substituirmos a equação (3.24) na formula de $a_{n n}^{I I} k$ obtemos:

$$
\begin{aligned}
a_{n n}^{I I}(k) & =-i\left\langle u_{n k}^{I I}\left|\nabla_{k}\right| u_{n k}^{I I}\right\rangle, \\
& =-i\left\langle u_{n,-k}^{I}\left|\Theta^{-1}(-) e^{-i \chi_{k n}} \nabla_{k}(-) e^{i \chi_{k n}} \Theta\right| u_{n,-k}^{I}\right\rangle, \\
& =-i\left\langle u_{n,-k}^{I}\left|\Theta^{-1} e^{-i \chi_{k n}} e^{i \chi_{k n}} \Theta\right| u_{n,-k}^{I}\right\rangle i \frac{\partial \chi_{n}}{\partial k}, \\
& -i\left\langle u_{n,-k}^{I}\left|\Theta^{-1} e^{-i \chi_{k n}} e^{i \chi_{k n}} \Theta \nabla_{k}\right| u_{n,-k}^{I}\right\rangle, \\
& =a_{n n}^{I}(-k)+\frac{\partial \chi_{n k}}{\partial k} .
\end{aligned}
$$

Agora podemos calcular a polarização parcial $P^{I}$ dada por:

$$
\begin{aligned}
P^{I} & =\frac{1}{2 \pi} \sum_{n}\left(\int_{0}^{\pi} d k a_{n n}^{I}(k)+\int_{-\pi}^{0} d k a_{n n}^{I}(k)\right)=\frac{1}{2 \pi} \sum_{n} \int_{0}^{\pi} d k\left(a_{n}^{I}(k)+a_{n}^{I}(-k)\right), \\
& =\frac{1}{2 \pi} \sum_{n} \int_{0}^{\pi} d k\left(a_{n n}^{I}(k)+a_{n n}^{I I}(k)-\frac{\partial \chi_{n k}}{\partial k}\right), \\
& =\int_{0}^{\pi} A(k)-\sum_{n} \frac{1}{2 \pi}\left[\chi_{\pi}-\chi_{0}\right] .
\end{aligned}
$$

Note que nos pontos TRIMs a matriz omega tem a seguinte propriedade $\omega_{n m}(\Lambda)=$ $-\omega_{m n}(\Lambda)$ (visto no apêndice F) e podemos escrever:

$$
\begin{aligned}
\omega_{1,2}\left(\Lambda_{i}\right) \omega_{3,4}\left(\Lambda_{i}\right) \cdot \omega_{2 N-1,2 N}\left(\Lambda_{i}\right) & =e^{\sum_{n}^{N} \chi_{n}\left(\Lambda_{i}\right)} \\
& =\operatorname{Pf}\left[\omega\left(\Lambda_{i}\right)\right] .
\end{aligned}
$$

O Pfaffiano é definido apenas para uma matriz antisimétrica. Para uma dada matriz $A$ o Pfaffiano está relacionado com o determinante da seguinte forma:

$$
P f^{2}[A]=\operatorname{det}[A]
$$

Desta forma podemos escrever:

$$
P 1=\int_{0}^{\pi} \frac{d k}{2 \pi} A(k)-\frac{i}{2 \pi} \log \left(\frac{\operatorname{Pf}[\omega(\pi)]}{\operatorname{Pf}[\omega(0)]}\right) .
$$

Agora podemos calcular o $P_{\Theta}$ da seguinte forma:

$$
\begin{aligned}
P_{\Theta} & =2 P^{I}-P_{\rho} \\
& =\int_{0}^{\pi} \frac{d k}{\pi} A(k)-\frac{i}{\pi} \log \left(\frac{P f[\omega(\pi)]}{\operatorname{Pf}[\omega(0)]}\right)-\int_{-\pi}^{\pi} \frac{d k}{2 \pi} A(k), \\
& =\int_{0}^{\pi} \frac{d k}{\pi} A(k)-\int_{0}^{\pi} \frac{d k}{2 \pi} A(k)-\int_{0}^{\pi} \frac{d k}{\pi} A(-k)-\frac{i}{\pi} \log \left(\frac{P f[\omega(\pi)]}{\operatorname{Pf}[\omega(0)]}\right), \\
& =\int_{0}^{\pi} \frac{d k}{\pi}[A(k)-A(-k)]-\frac{i}{\pi} \log \left(\frac{P f[\omega(\pi)]}{\operatorname{Pf}[\omega(0)]}\right) .
\end{aligned}
$$

Assim com $A(k)=\operatorname{tr}(a(k))$ e usando as propriedades da matriz $\omega$ vistas no apêndice $\mathrm{F}$ obtemos:

$$
\begin{aligned}
P_{\Theta} & =\int_{0}^{\pi} \frac{d k}{2 \pi} \operatorname{itr}\left[\omega^{\dagger}(k) \frac{\partial}{\partial k} \omega(k)\right]-\frac{i}{\pi} \log \left(\frac{P f[\omega(\pi)]}{\operatorname{Pf}[\omega(0)]}\right) \\
& =\int_{0}^{\pi} \frac{d k}{2 \pi} \frac{\partial}{\partial k} \log (\operatorname{det}[\omega(k)])-\frac{i}{\pi} \log \left(\frac{P f[\omega(\pi)]}{\operatorname{Pf}[\omega(0)]}\right), \\
& =\frac{i}{2 \pi} \log \left(\frac{\operatorname{det}[\omega(\pi)]}{\operatorname{det}[\omega(0)]}\right)-\frac{i}{\pi} \log \left(\frac{P f[\omega(\pi)]}{\operatorname{Pf}[\omega(0)]}\right), \\
& =\frac{1}{i \pi} \log \left(\frac{\sqrt{\operatorname{det}[\omega(0)]} \operatorname{Pf}[\omega(\pi)]}{\sqrt{\operatorname{det}[\omega(\pi)]} \operatorname{Pf}[\omega(0)]}\right)=\frac{1}{i \pi} \log \left(\frac{P f[\omega(\pi)] P f[\omega(0)]}{\sqrt{\operatorname{det}([\omega(\pi)])} \sqrt{\operatorname{det}([\omega(0)])}}\right) .
\end{aligned}
$$


O argumento deste log é \pm 1 . Como $\log (-1)=i \pi, P_{\Theta}=0$, ou 1. Fisicamente, os dois valores de $P_{\Theta}$ correspondem a dois estados de polarização que o sistema (aqui unidimensional) se encontra em cada TRIM diferente. Para se observar uma mudança de polarização depois de certo tempo $T$ onde o sistema volta a seu estado inicial é definido um invariante $Z_{2}=P_{\Theta}(k, t=T)-P_{\Theta}(k, t=0)$. Aqui reinterpretando o espaço periódico em 2D $(k, t)$, o qual forma um torus, como uma zona de Brillouin em duas dimensões $\left(k_{x}, k_{y}\right)$, a teoria acima permite uma classificação topológica via $Z_{2}$ para um sistema com $2 N$ bandas ocupadas. Assim podemos escrever $Z_{2}=P_{\Theta}\left(k_{x}, k_{y}=G_{y} / 2\right)-P_{\Theta}\left(k_{x}, k_{y}=0\right)$ ou $Z_{2}=P_{\Theta}\left(k_{x}=G_{x} / 2, k_{y}\right)-P_{\Theta}\left(k_{x}=0, k_{y}\right)$. Qualquer uma destas igualdades proporciona o seguinte:

$$
(-1)^{Z_{2}}=\prod_{i=1}^{T R I M s} \frac{\operatorname{Pf}\left[\omega\left(\Lambda_{i}\right)\right]}{\sqrt{\operatorname{det}\left[\omega\left(\Lambda_{i}\right)\right]}} .
$$

A consequência de dois estados de polarização diferentes pode ser vista na figura 7 .
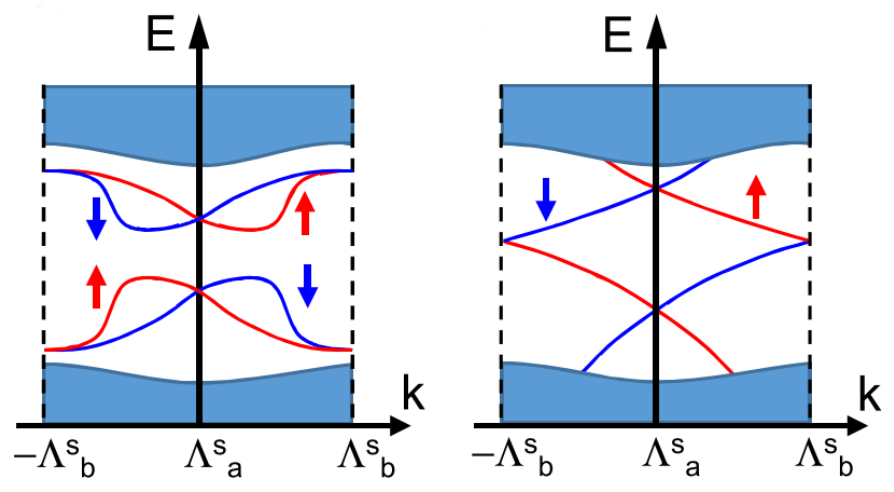

Figura 7: Estruturas de bandas esquemáticas de duas bordas nos limites dos pontos TRIMs $\Lambda_{a}^{s}$ e $\Lambda_{b}^{s}$ para um isolante trivial(esquerda) e um topológico do tipo $Z_{2}$ (direita). As regiões em azul representam a zona de bulk e para aumentar a visibilidade foi feita um dispersão entre $-\Lambda_{b}^{s}$ a $\Lambda_{b}^{s}$. No isolante topológico temos uma mudança da polarização de reversão temporal, efeito que pode ser visto como uma troca de centros de carga de Wannier, efeito explicado no apêndice $H$. Imagem da referência [63].

\subsection{Extensão para 3D}

Três grupos teóricos diferentes independentemente descobrem que a caracterização topológica do efeito Hall quântico de spin tem uma generalização em três dimensões [1214], e deram o nome de isolantes topológicos. Fu, Kane e Mele propõem alguns canditados 
reais a pertencerem a esta nova classe topológica, dentre eles ligas de $\mathrm{Bi}_{1-x} \mathrm{Sb}_{x}$, poços quânticos de HgTe nos quais se aplica pressão e $\alpha$-Sn. Apenas em 2008 temos a observação experimental do primeiro isolante topológico, a liga $\mathrm{Bi}_{1-x} \mathrm{Sb}_{x}$ [15]. Em 2009, os isolantes topológicos de segunda geração ou estequiométricos da família $(\mathrm{Bi}, \mathrm{Sb})_{2}(\mathrm{Se}, \mathrm{Te})_{3}$, foram teoricamente [17] e experimentalmente $\left(\mathrm{Bi}_{2} \mathrm{Se}_{3}\right.$ [75-77], $\mathrm{Bi}_{2} \mathrm{Te}_{3}$ [18,76, 78,79], $\mathrm{Sb}_{2} \mathrm{Te}_{3}$ [79]) identificados. Tais materiais possuem características interessantes descritas no capítulo posterior.

Na zona de Brillouin em três dimensões, os seis planos, $x=0, x= \pm \pi, y=0, y= \pm \pi$, $z=0, z= \pm \pi$, possuem as mesmas simetrias que a zona de Brillouin em apenas duas dimensões, portante possuem um $Z_{2}$ atrelado a cada plano. Porém os seis invariantes não são independentes. Isto se explica porque $x_{0} x_{1}=y_{0} y_{1}=z_{0} z_{1}$, e isso vem do fato que todos são produtos de $\frac{P f\left[\omega\left(\lambda_{i}\right)\right]}{\sqrt{\operatorname{det}\left[\omega\left(\lambda_{i}\right)\right]}}$ dos 8 TRIMs. Logo, eliminando 2 deles, obtemos 4 invariantes topológicos. A construção proposta por Fu, Kane e Mele [12] é propor um $\delta\left(\Lambda_{i}\right)$, onde:

$$
\delta\left(\Lambda_{i}\right)=\frac{\operatorname{Pf}\left[\omega\left(\lambda_{i}\right)\right]}{\sqrt{\operatorname{det}\left[\omega\left(\lambda_{i}\right)\right]}} .
$$

Assim os quatro invariantes $Z_{2}$ são dados por $\left(\nu_{0}, \nu_{1} \nu_{2} \nu_{3}\right)$ visto na figura 50 e definidos como:

$$
\begin{aligned}
& (-1)^{\nu_{0}}=\prod_{n_{j}=0, \pi} \delta\left(\Lambda_{n_{1}}, \Lambda_{n_{2}}, \Lambda_{n_{3}}\right) \\
& (-1)^{\nu_{i}}=\prod_{n_{j \neq i}=0, \pi ; n_{i}=\pi} \delta\left(\Lambda_{n_{1}}, \Lambda_{n_{2}}, \Lambda_{n_{3}}\right)(i=1,2,3) .
\end{aligned}
$$

O conjunto $\left(\nu_{1} \nu_{2} \nu_{3}\right)$, representam uma mudança de polarização dentro de um mesmo plano, mas não possuem a mesma proteção da TRS do que o invariante $\nu_{0}$. Este trio de invariantes não nos interessa pois dependem da escolha da rede recíproca. O invariante $\nu_{0}$ é o produto sobre todos os $\delta\left(\Lambda_{i}\right)$ em cada um dos oito TRIMs, ele é único para um material em três dimensões, e muitas vezes comparado ao número $Z_{2}$, em duas dimensões, possuindo significado equivalente. Este invariante não depende da escolha dos seus vetores da rede recíproca. Um material $\operatorname{com} \nu_{0}=0$ é classificado como topologicamente trivial, já $\nu_{0}=1$ trata-se de um isolante topológico que apresenta na sua superfície o efeito Hall quântico de spin. É o invariante $\nu_{0}$ que determina de que maneira os pontos de Kramer degenerados ou TRIMs irão cruzar o nível de Fermi. Um número ímpar (par) de 
cruzamentos representa um material com $\nu=1(\nu=0)$.

O primeiro isolante topológico $\mathrm{Bi}_{1-x} \mathrm{Sb}_{x}$ pode ser descrito como $(1,111)$. Ao substituir Bi por Sb muda-se a banda de energia. Na sua fase bulk, para $x \simeq 0.04$ o gap se fecha, e ao se aumentar a concentração acima de $x \simeq 0.07$ temos um ordenamento de bandas invertido. Ao aumentar a concentração além de $x \simeq 0.22$ o nível de Fermi sobe acima da banda de condução retornando a um caráter semi-metálico, como visto na figura 8. As
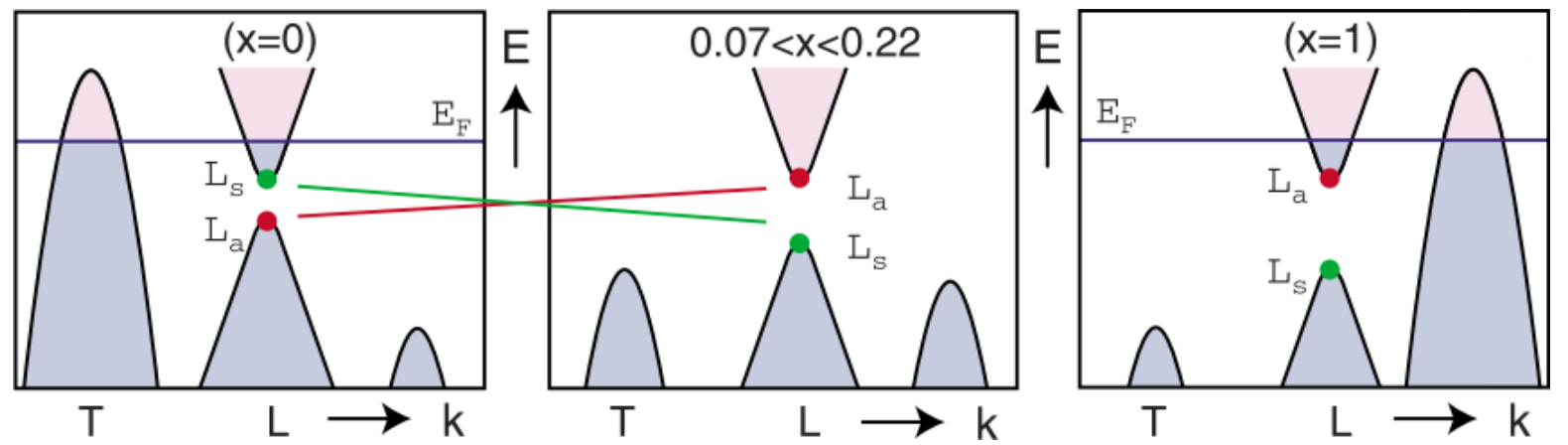

Figura 8: Representação esquemática da estrutura de banda do material $B i_{1-x} S b_{x}$ em sua fase bulk. Este material muda de um comportamento semi-metal para $x<0.07$, para um comportamento topológico para $0.07<x<0.22$ e de volta para um comportamento semi-metal para $x>0.22$. As bandas de condução $L_{s}$ e valência $L_{a}$ invertem para $x \simeq 0.04$. Imagem da referência [62].

classificações topológicas em três dimensões podem ser vistas na figura 9. A estrutura de banda eletrônica de $\mathrm{Bi}_{1-x} \mathrm{Sb}_{x}$ na superfície cruza o nível de Fermi 5 vezes [76] (3 vezes [80]) entre os seus pontos TRIMs, sendo um isolante topológico. Todavia seu gap de energia no bulk ( $\simeq 30 \mathrm{meV}[16])$ é muito pequeno para aplicação em dispositivos. Além disso, em uma liga existe uma dificuldade intrínseca para se obter um alto grau de pureza na concentração desejada. Sua superfície complicada como evidenciada por alguns trabalhos $[76,80]$, que não concordam completamente com cálculos teóricos de primeiros princípios $[81,82]$. Essas complicações mudaram o interesse desta área para os isolantes topológicos de segunda geração. $\mathrm{Na}$ família de materiais $(\mathrm{Bi}, \mathrm{Sb})_{2}(\mathrm{Se}, \mathrm{Te})_{3}$, com a exceção de $\mathrm{Sb}_{2} \mathrm{Se}_{3}$, todos possuem uma única inversão de banda na fase bulk, no ponto $\vec{k}=0$ (também chamado de ponto $\Gamma$ ) levando a uma classificação do tipo (1,000). $\mathrm{Bi}_{2} \mathrm{Se}_{3}$ dentre os outros integrantes da mesma família de materiais tornou-se referência na área de isolantes topológicos por três motivos: 
- Concordância entre medidas de ARPES e cálculos teóricos evidenciando um único cone de Dirac no ponto $\Gamma$ do espaço recíproco, como mostrados nas referências acima.

- Este material é estequiométrico permitindo um alto grau de pureza.

- Gap no bulk da ordem de $0.3 \mathrm{eV}$, indicando que $\mathrm{Bi}_{2} \mathrm{Se}_{3}$ poderia exibir comportamento topológico em temperatura ambiente.

A descoberta de grande parte dos materiais topológicos foi via cálculo do invariante $Z_{2}$ pelo método da avaliação dos valores esperados do operador paridade elaborado por $\mathrm{Fu}$ e Kane [67], feito e descrito no apêndice D no formalismo de ondas planas. O cálculo do invariante $Z_{2}$ ou $\nu_{0}$ continua sendo a maneira mais robusta de descrever a topologia dos materiais. Para se entender melhor os resultados vamos analisar no próximo capítulo os materiais estudados no corpo desta tese.

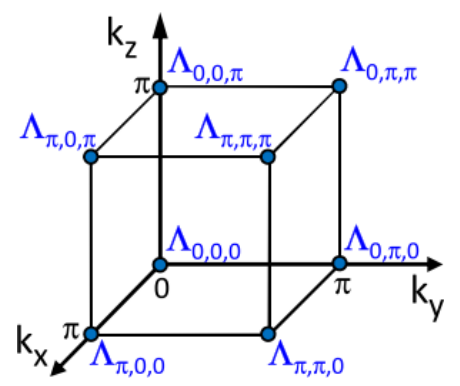

(a) TRIMs da ZB em $3 D$.

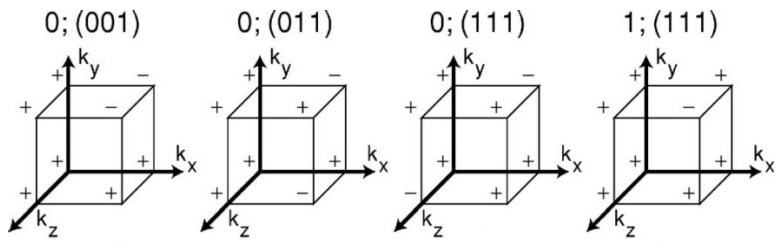

(b) Diferentes fases topológicas.

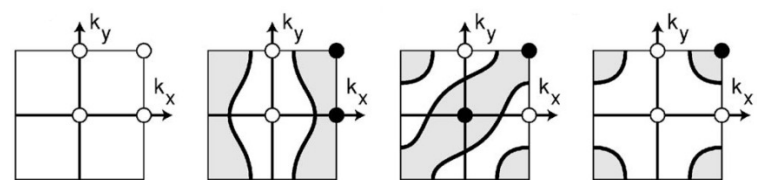

(c) Estrutura de banda para cada fase.

Figura 9: (a) Extensão para 3D dos pontos TRIMs da zona de Brillouin. (b) - (c) Diagramas mostrando as 4 diferentes fases indexadas por $\left(\nu_{0}, \nu_{1} \nu_{2} \nu_{3}\right)$ (b) Sinais de $\delta_{i}$ nos pontos $\Gamma_{i}$ nos vértices do cubo (c) Estrutura de Banda de uma superfície 001 para cada fase. As linhas grossas indicam os possiveis cruzamentos das bandas no nivel de Fermi. Os círculos abertos e fechados representam a polarização de reversão temporal para cada TRIM de superfície $\Lambda_{a}$. Imagem da referência [63]. 
$\longdiv { \text { capituo } } 4$

\section{Isolantes Topológicos em 3 e 2 dimensões}

O passo inicial para utilizar os métodos de primeiros princípios é obter uma boa descrição das estruturas de cada sistema. Neste capítulo abordamos os isolantes topológicos da família de $(\mathrm{Bi}, \mathrm{Sb})_{2}(\mathrm{Te}, \mathrm{Se})_{3}$, cuja tese relata as propriedades eletrônicas e magnéticas além de descrever o Germaneno funcionalizado um isolante topológico em duas dimensões. Uma análise sistemática de superfícies nesta família de materiais, $\mathrm{Bi}_{2} \mathrm{Se}_{3}$ [75, 76, 83], $\mathrm{Bi}_{2} \mathrm{Te}_{3}[18,76,78,79]$ e $\mathrm{Sb}_{2} \mathrm{Te}_{3}$ [79], Ge-X com [H,F,Cl,Br,I] [24], comprova suas estruturas de banda topológicas. Nestes trabalhos foi confirmado que o comportamento topológico está associado a uma inversão de banda no ponto $\Gamma(\vec{k}=0)$, levando a classe topológica $(1,000)$ em três dimensões e $Z_{2}=1$ em duas dimensões, descrita no capítulo anterior. Em uma primeira etapa vamos detalhar a geometria destas estruturas, e em seguinda descreveremos algumas de suas propriedades eletrônicas exóticas.

\subsubsection{Propriedades geométricas}

A família $(\mathrm{Bi}, \mathrm{Sb})_{2}(\mathrm{Te}, \mathrm{Se})_{3}$ possui uma estutura cristalina romboédrica do grupo espacial $D_{3 d}{ }^{5}$. O sistema tem como célula unitária cinco camadas atômicas, que pertencem a três grupos de camadas também contendo cinco átomos chamados de Quintuple Layers (QLs) diferentes como visto na figura 10. As ligações entre as camadas (QLs) são muito mais fracas devido as interações de Van der Walls. Os vetores primitivos são dados pelos 


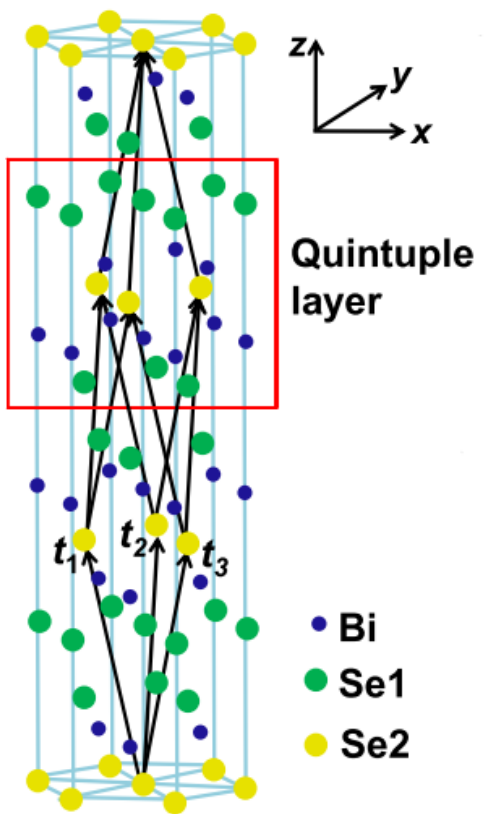

(a) Supercélula de $\mathrm{Bi}_{2} \mathrm{Se}_{3}$.

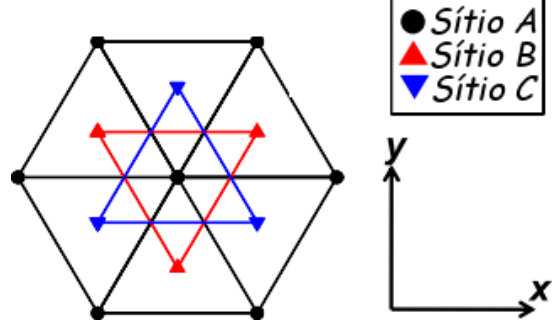

(b) Recorte no eixo z da rede real.

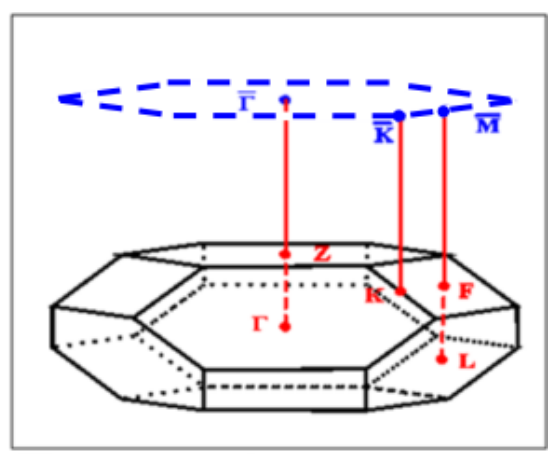

(c) $Z B$ em $3 D$ e sua superfície $2 D$.

Figura 10: (a) Supercélula hexagonal de $B i_{2} S e_{3}$, contendo 15 camadas atômicas mais os vetores primitivos da rede, (b) Recorte no eixo $z$ da rede triangular, onde vemos que há três diferentes tipos de sítios não equivalentes (c) Primeira zona de Brillouin contendo 4 pontos TRIM (TimeReversal-Invariant-Momentum) e a superfície $2 D$ (projeção da zona em 3D) da zona de Brillouin que está representada em linhas azuis. Imagem da referência [84].

vetores da equação (4.1).

$$
t_{1,2,3}=\left\{\begin{array}{l}
t_{1}=\left(\frac{-a}{2}, \frac{-\sqrt{3} a}{6}, \frac{c}{3}\right) \\
t_{2}=\left(\frac{a}{2}, \frac{-\sqrt{3} a}{6}, \frac{c}{3}\right) \\
t_{3}=\left(0, \frac{\sqrt{3} a}{3}, \frac{c}{3}\right)
\end{array} .\right.
$$

Aqui $a$ e $c$, são parâmetros de rede na célula hexagonal. Logo os correspondentes vetores da rede recíproca $s_{1,2,3}$, definidos de forma que $\overrightarrow{s_{i}} \cdot \overrightarrow{t_{j}}=2 \pi \delta_{i j}$, são dados pela equação (4.2), onde $b=\frac{a}{c}$ e $h=\frac{2 \pi}{a}$.

\begin{tabular}{cccccc}
\hline & & $\mathrm{Sb}_{2} \mathrm{Te}_{3}$ & $\mathrm{Bi}_{2} \mathrm{Te}_{3}$ & $\mathrm{Bi}_{2} \mathrm{Se}_{3}$ & $\mathrm{Sb}_{2} \mathrm{Se}_{3}$ \\
\hline Lattice Constant & $\mathrm{a}(\AA)$ & 4.250 & 4.383 & 4.138 & - \\
& $\mathrm{c}(\AA)$ & 30.35 & 30.487 & 28.64 & - \\
inner Coordinates & $\mu$ & 0.400 & 0.400 & 0.399 & 0.399 \\
& $\nu$ & 0.211 & 0.212 & 0.206 & 0.206 \\
\hline
\end{tabular}

Tabela 1: Valores dos parâmetros a, c, $\mu$ e $\nu$ para diferentes elementos da família do grupo $D_{3 d^{5}}$. 


$$
s_{1,2,3}=\left\{\begin{array}{l}
s_{1}=\frac{\left(-1, \frac{-\sqrt{3}}{3}, b\right)}{h} \\
s_{2}=\frac{\left(1, \frac{-\sqrt{3}}{3}, b\right)}{h} \\
s_{3}=\frac{\left(0, \frac{2 \sqrt{3}}{3}, b\right)}{h}
\end{array} .\right.
$$

Dada a tabela 1 vamos olhar o caso do $\mathrm{Bi}_{2} \mathrm{Se}_{3}$. Como vemos na figura 10 para criar a célula primitiva ou a QL, inserimos um átomo de Se na origem $(0,0,0)$, que chamaremos de Selênio tipo zero, a seguir inserimos dois átomos de Bi nas posições $( \pm \mu, \pm \mu, \pm \mu)$, que chamaremos de Bismuto tipo um, e finalmente inserimos dois átomos de Se nas posições $( \pm \nu, \pm \nu, \pm \nu)$, que chamaremos de Selênio tipo dois. Pode-se mostrar, utilizando teoria de grupos, que possíveis degenerescências na banda de dispersão eletrônica que levam a fenômenos interessantes acontecem em pontos de alta simetria na zona de Brillouin. Logo é necessário olhar quais são os pontos de alta simetria. Na figura 10c vemos a zona de Brillouin 3D, e a superfície 2D, que representa uma fatia da zona 3D, ou uma projeção quando olharmos fatias desses materiais. Temos aqui 4 pontos importantes na zona em 3D, $\Gamma(0,0,0), L\left(\frac{1}{2}, 0,0\right), F\left(\frac{1}{2}, \frac{1}{2}, 0\right)$ e $Z\left(\frac{1}{2}, \frac{1}{2}, \frac{1}{2}\right)$, todos escritos em função dos vetores da rede recíproca. Estes pontos são invariantes sob inversão temporal (TRIM) e são importantes para a descrição do material. Na zona 2D, os pontos $\Gamma$ e $Z$ são projetados em $\bar{\Gamma} ; L$ e $F$, são projetados em $\bar{M}$, além disso temos o ponto $\bar{K}$, onde este último não é um TRIM.

Para se estudar efeitos de superfície, existe a necessidade de recortar o bulk criado para gerar fatias com a inclusão do vácuo. Ao recortar o material, obtemos algumas modificações vista na figura 11. Agora nossa célula unitária tem 3QL com 15 átomos, e nossos vetores da rede direta passam a ter a formato da equação (4.3).

$$
t_{1,2,3}^{\text {superficie }}=\left\{\begin{array}{l}
t_{1}^{\text {sup }}=(a, 0,0) \\
t_{2}^{\text {sup }}=\left(\frac{a}{2}, \frac{\sqrt{3} a}{2}, 0\right) . \\
t_{3}^{\text {sup }}=\left(0,0, \frac{c}{2}\right)
\end{array}\right.
$$

Nesta tese outro material estudado foi o Germaneno, cuja estrutura de bulk em duas dimensões pode ser vista na figura 12a. Este sistema faz parte do grupo $D_{6 h}$. Sua estrutura geométrica é muito similar à do grafeno e siliceno, apresentando um pequeno "buckling" como vista na figura 12b. A rede de Brillouin pode ser vista na figura 12c. 


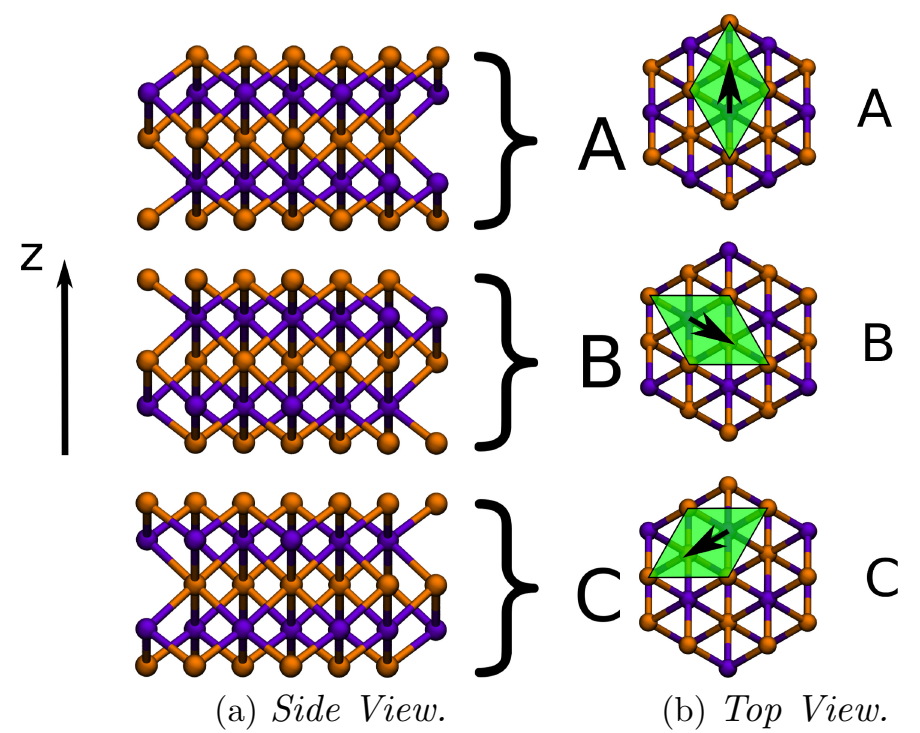

Figura 11: Representação estrutural de $\mathrm{Bi}_{2} \mathrm{Se}_{3}$ e $B i_{2} \mathrm{Te}_{3}$. (a) Visão Lateral das 3 QL inequivalentes. (b) Visão panorâmica de cada $Q L A, B$ e C, individualmente. As células em verde com suas respectivas setas pretas representam uma orientação fictícia, na idéia de mostar sua equivalência via uma rotação $C_{3}$.

Neste sistema os vetores da rede direta são dados pela equação (4.4).

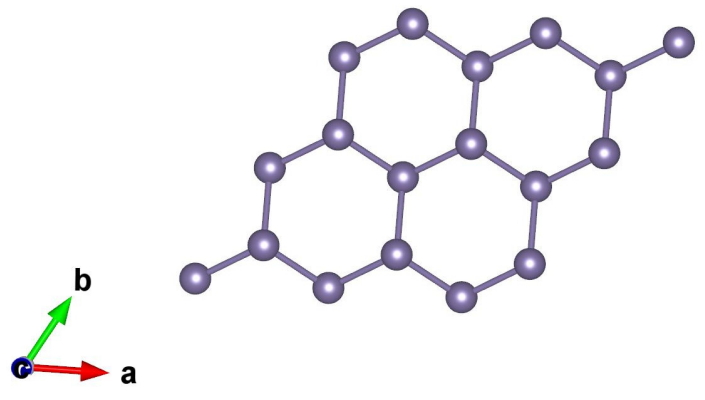

(a) Visão panorâmica.

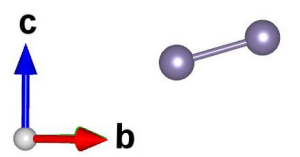

(b) Visão lateral.

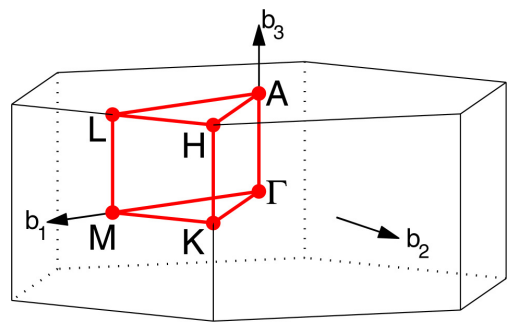

(c) $Z B$ em $3 D$.

Figura 12: Visão panorâmica (a) e lateral (b) da folha de Germaneno, onde se nota uma similaridade com a geometria do grafeno, porém com um "buckling" orientado no eixo z. Em (c) temos a primeira zona de Brillouin desta estrutura hexagonal com os pontos de altas simetria e seus caminhos em vermelho.

$$
h_{1,2,3}=\left\{\begin{array}{l}
h_{1}=\left(a \frac{\sqrt{(3)}}{2}, \frac{1}{2}, 0\right) \\
h_{2}=\left(a \frac{\sqrt{(3)}}{2},-\frac{1}{2}, 0\right) \\
h_{3}=(0,0, c)
\end{array}\right.
$$

A célula primitiva é dada por 2 átomos localizados em $(0,0,0)$ e $\left(\frac{a \sqrt{(3)}}{3}, 0,0.69\right)$ no eixo 
cartesiano direto. Os pontos de alta simetria escritos em função da rede recíproca são $\Gamma(0,0,0), M\left(\frac{1}{2}, \frac{1}{2}, 0\right), K\left(\frac{2}{3}, \frac{1}{3}, 0\right)$. Neste sistema também é possível estudar efeitos de borda para isso criamos uma supercélula com distância suficiente entre cada borda para não ocorrer interação. Note que nestes sistemas a borda pode ser recortada de duas maneiras, tanto em Zigzag, como em Armchair, ambas descritas na figura 13. Na tese trabalhamos

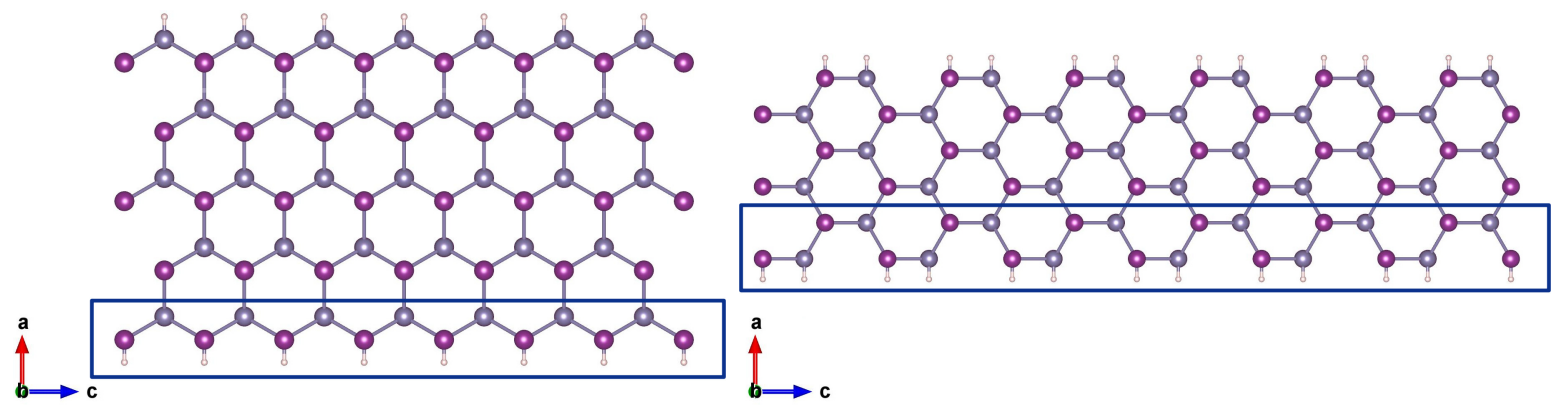

(a) Nanofita ZigZag.

(b) Nanofita Armchair.

Figura 13: Representação de dois possiveis recortes de fita do Germaneno funcionalizado com iodo e saturado com hidrogênio nas pontas. Cada recorte esta sinalizado em um quadro azul senda (a) um recorte do tipo Zigzag, e (b) um outro do tipo Armchair.

também com o Germaneno funcionalizado com halogênios e o formato dos vetores de rede são os mesmos, havendo apenas a soma de dois átomos na base, como visto na figura 14 . Com as características geométricas descritas podemos analisar as propriedades eletrônicas

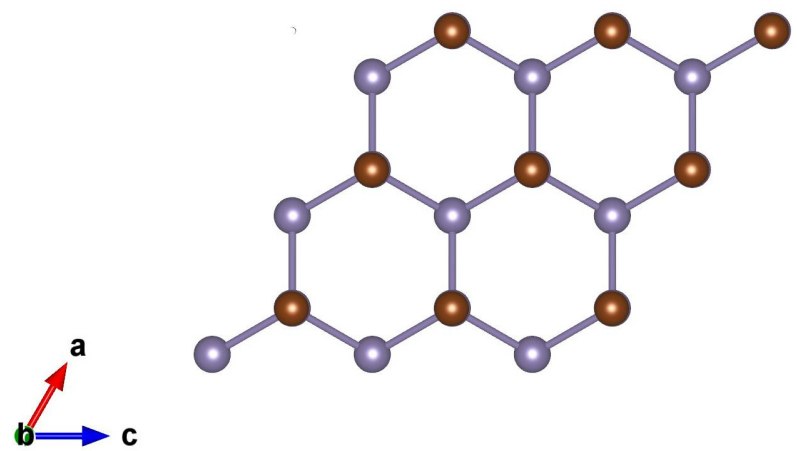

(a) Visão panorâmica.

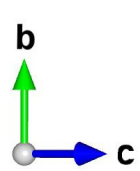

(b) Visão lateral.

Figura 14: Visão panorâmica (a) e lateral (b) do Germaneno funcionalizado com iodo.

destes sistemas.

\subsubsection{Propriedades eletrônicas}

Sabemos que um material é um isolante topológico $Z_{2}$ quando sua topologia é não trivial. No caso do $\mathrm{Bi}_{2} \mathrm{Se}_{3}$ a não trivialidade de sua topologia vem de uma inversão de 
bandas no ponto $\Gamma$. Neste material como não há forte hibridização podemos montar um modelo de níveis atômicos como visto na figura 15a. Vemos que ao se considerar as ligações químicas, simetria de inversão, campo cristalino, e a interação spin órbita, obtemos que o orbital $p_{z}$ proveniente do átomo de Bismuto tipo um inverte com o orbital $p_{z}$ do átomo de Selenio tipo dois. Note nas bandas das figuras $15 \mathrm{~b}$ e $15 \mathrm{c}$ que quando se considera a interação spin órbita existe uma única inversão no ponto $\Gamma$, ou seja em um número impar de pontos na zona de Brillouin, criando um gap na fase bulk que chamamos de não trivial. Buscando entender o papel da proximidade das superfícies, criamos superfícies

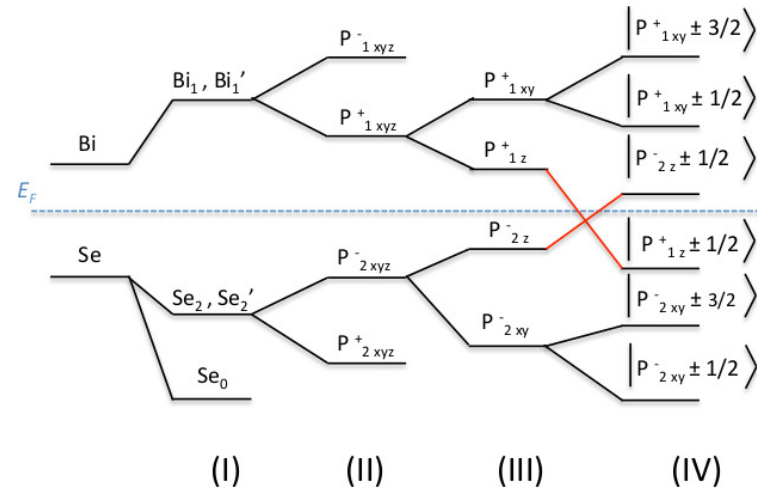

(a) Evolução dos orbitais atômicos $P_{x, y, z}$.

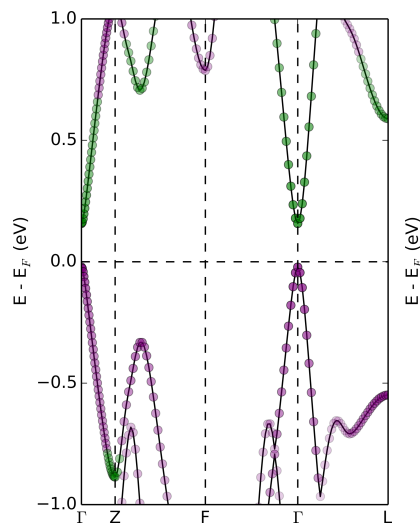

(b) Banda sem SOC.

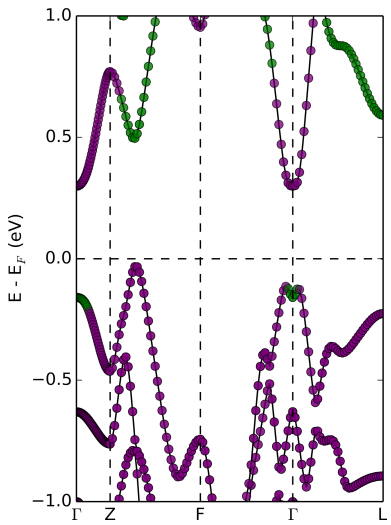

(c) Banda com $S O C$.

Figura 15: Em (a) temos um diagrama esquemático da evolução dos orbitais atômicos $P_{x, y, z}$ de Bi e Se nas bandas de valência e condução de $B i_{2} S_{3}$ no ponto $\Gamma$. Os estagios (I), (II), (III) e (IV) representam respectivamente o efeito de se considerar as ligações químicas, simetria de inversão, campo cristalino, e a interação spin órbita. Em azul temos a energia de Fermi. Em (b) e (c) temos as bandas de bulk do sistema $\mathrm{Bi}_{2} \mathrm{Se}_{3}$ com e sem spin órbita. Em ambas as imagens temos em verde e roxo a projeção dos orbitais $p_{z}$ dos átomos de Bi e Se. Nota-se que existe uma inversão de bandas perto do ponto $\Gamma$ quando a interação spin órbita é considerada.

(adicionando $20 \AA$ de vácuo) para o caso $\mathrm{Bi}_{2} \mathrm{Se}_{3}$ com diferentes números de QLs (variando de um à seis) e plotamos suas bandas de dispersão de energia com e sem interação spin órbita vistas na figura 16. Aqui podemos ver que a interação spin órbita é importante para a inversão de bandas e criação de estados metálicos. A inexistência de estados metálicos em filmes finos de $\mathrm{Bi}_{2} \mathrm{Se}_{3}$ está associada com a proximidade de superfícies com helicidades opostas que hibridizam, via tunelamento quântico, de forma a quebrar o invariante $Z_{2}$ e abrir um pequeno gap. Para o caso do Germaneno efeito similar ocorre ao se considerar a interação spin órbita. Porém aqui a inversão é da paridade dos orbitais $p_{z}$ no ponto $K$ da 

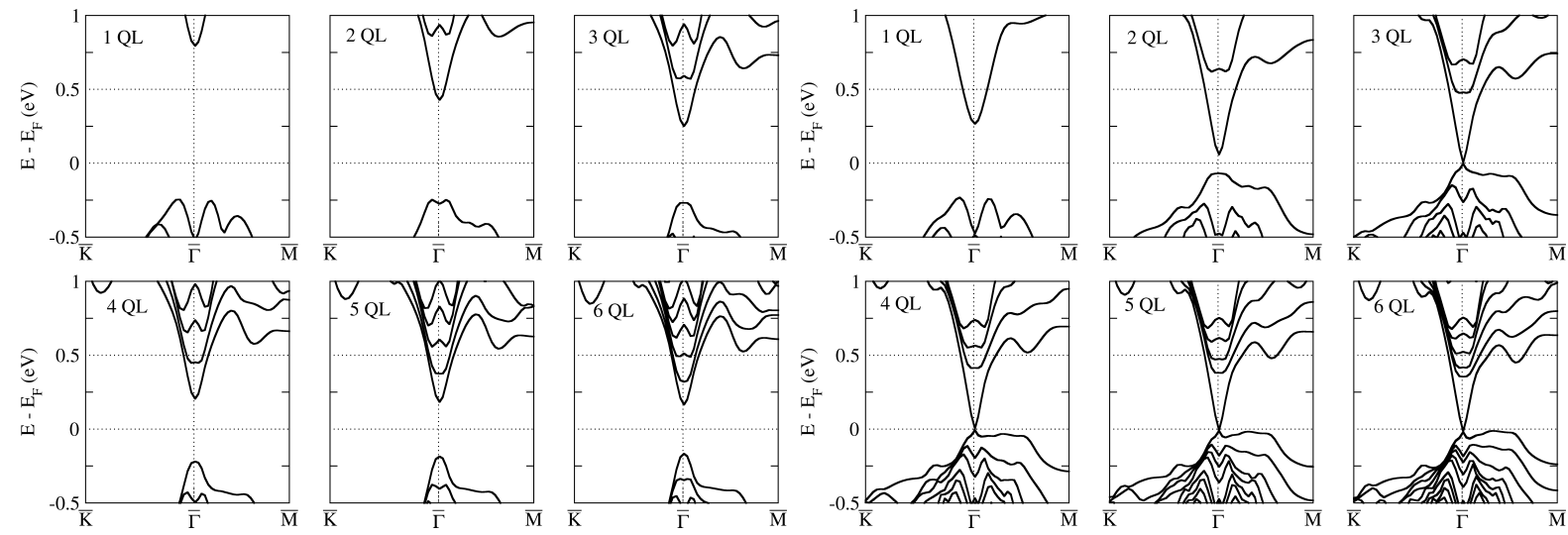

(a) Bandas sem SOC.

(b) Bandas com SOC.

Figura 16: (a) - (b) Dispersões de bandas da superfície do sistema $B i_{2} S e_{3}$ para vários tamanhos de $Q L$, sem e com interação spin órbita. Nota-se que sem interação spin órbita não há formação de estados metálicos e além disso, apenas a partir de 4QL os estados metálicos aparecem.

zona de Brillouin, ou seja ao ligar esta interação os orbitais ligantes e anti-ligantes, antes degenerados se invertem em relação ao vácuo, gerando uma topologia não trivial como visto na figura 17. Quando funcionalizamos o sistema, as ligações $p_{z}$ antes delocalizadas,

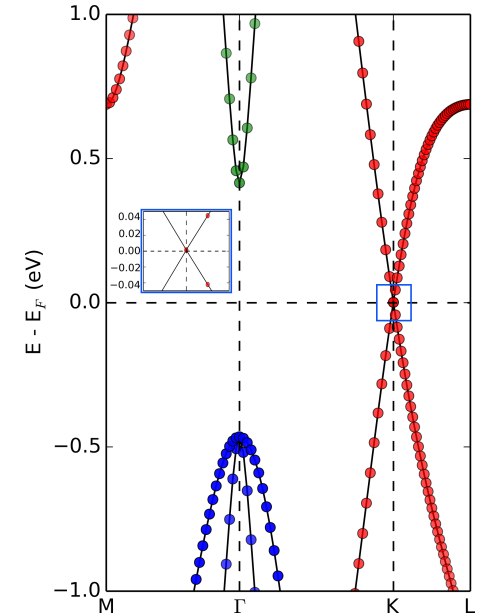

(a) Banda sem SOC.

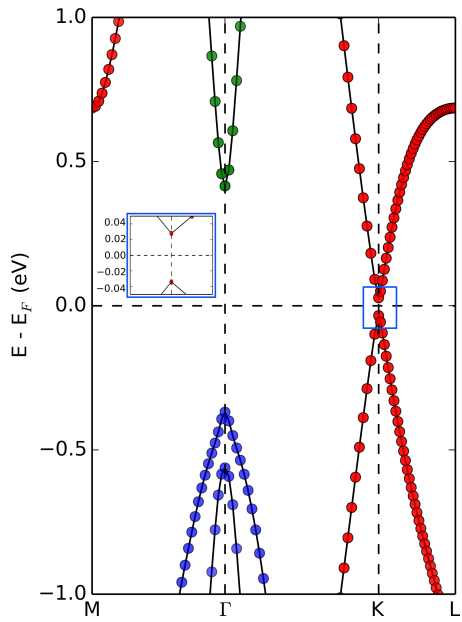

(b) Banda com SOC.

Figura 17: Em (a) - (b) temos respectivamente bandas projetadas nos orbitais do germanio do sistema Germaneno pristino sem e com spin órbita. Em cada figura temos em verde, azul e vermelho as projeções nos orbitais $s, p_{x}+p_{y}$, e $p_{z}$. Próximo ao ponto $K$ temo um inset mostrando a abertura da degenerescência no ponto $K$ ao se incluir spin órbita, que representa a inversão de paridade da banda.

passam a ser parte de uma ligação $\sigma$ muito mais forte. Todavia ainda temos uma inversão agora no ponto $\Gamma$ na qual bandas de paridades diferentes do tipo $p_{x y}$ estão invertidas como 
visto na figura 18. Tendo caracterizado os materiais podemos abordar os resultados.

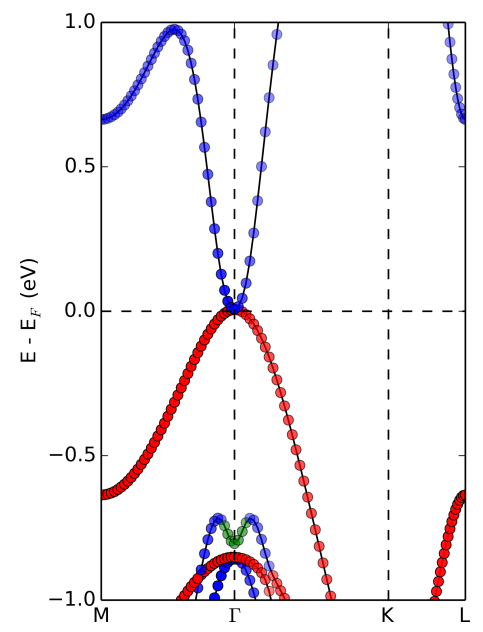

(a) Banda sem $S O C$.

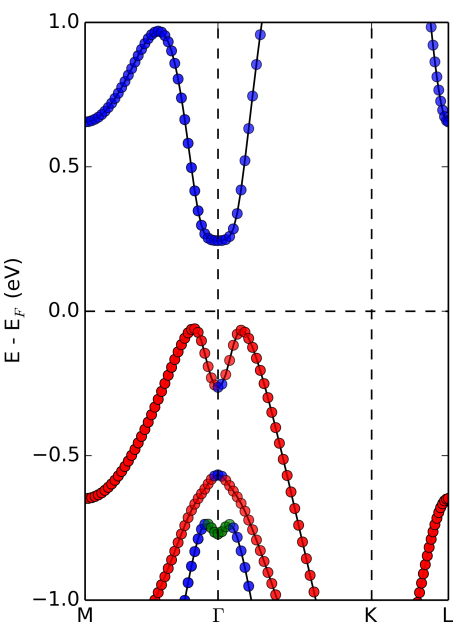

(b) Banda com SOC.

Figura 18: Em (a) - (b) temos o mesmo que para a figura 17, para o Germaneno funcionalizado com o Iodo. Aqui a inversão de orbitais do tipo $p_{x y}$ ocorre no ponto $\Gamma$. Logo espera-se um estado metálico na borda neste ponto da zona de Brillouin. 


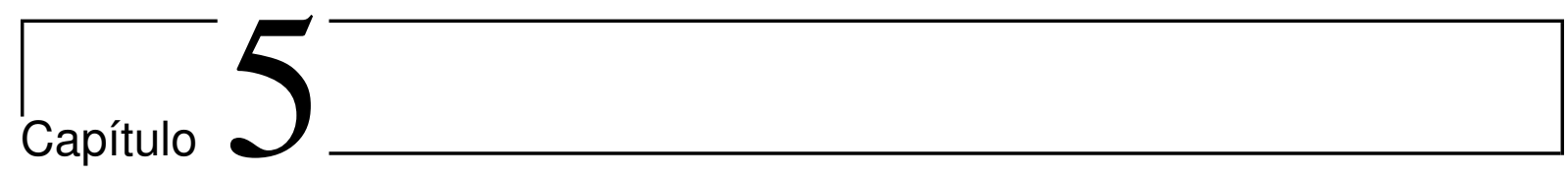

\section{Falhas de empilhamento em Isolantes}

\section{topológicos fortes}

Sabe-se que a estrutura e simetria dos átomos em $\mathrm{Bi}_{2} \mathrm{Se}_{3}$ e $\mathrm{Bi}_{2} \mathrm{Te}_{3}$, tem um papel central nas propriedades eletrônicas destes materiais. Vimos no capítulo anterior que para filmes finos abaixo de 4 QL, há a formação de um gap de energia, representando uma mudança de fase topológica. Análises experimentais e teóricas [77, 85] vistas na figura 19 confirmam este fenômeno. Este efeito está relacionado com a proximidade entre as duas superfícies, evidenciado também pela variação do espaçamento de Van Der Waals, que passa a ser um divisor entre isolante topológico e trivial. Além disso ligas
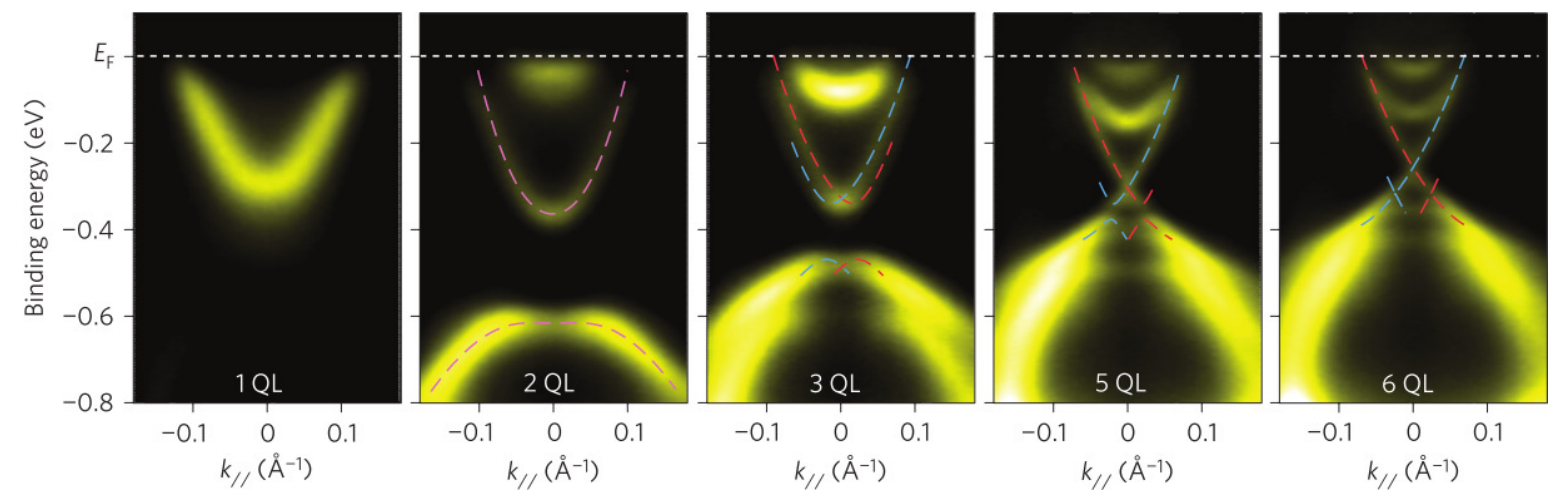

Figura 19: Medidas de ARPES para filmes finos de $B i_{2} S_{3}$ a temperatura ambiente ao longo da direção $\bar{\Gamma}-\bar{K}$. A curva em rosa em $2 Q L$ representa ajuste do modelo Bernevig-Hughes-Zhang do isolante Hall quântico de spin em duas dimensões. As curvas azuis e vermelhas em 3, 5 e 6 QL representam ajustes para o mesmo modelo com inclusão de Rashba splitting. Imagem da referência [85].

$\mathrm{Bi}_{2-x} \mathrm{Ca}_{x} \mathrm{Se}_{3}$ [76], estruturas dopadas $\mathrm{BiTiSe}_{3}$, [86] ou $\mathrm{Bi}_{2} \mathrm{Te}_{2} \mathrm{Se}$ [87], e também sistemas 
sob estresse mecânico [88], possuem respectivamente uma transição topológica de caráter estrutural para uma certa concentração, ou uma porcentagem de dopagem ou mesmo intensidade de estresse mecânico.

Baseados nestas informações, e no fato de que falhas de empilhamento estendidas na direção [001] da célula hexagonal de $\mathrm{Bi}_{2} \mathrm{Se}_{3}$ e $\mathrm{Bi}_{2} \mathrm{Te}_{3}$, foram reportadas experimentalmente [89,90], decidiu-se fazer uma investigação na família destes materiais do papel de falhas de empilhamento nas propriedades eletrônicas.

\subsection{Metodologia}

Foram feitos cálculos baseados em primeiros princípios, usando teoria do funcional da densidade [32, 34], nos isolantes topológicos $\mathrm{Bi}_{2} \mathrm{Se}_{3}$ e $\mathrm{Bi}_{2} \mathrm{Te}_{3}$. Para o potencial de troca e correlação foi considerada a aproximação de densidade local LDA [45], para cálculos de energia de ligação entre QLs, enquanto que cálculos de estrutura eletrônica foram efetuadas na aproximação de gradiente generalizado GGA [46]. A interação spin órbita foi tratada de maneira auto consistente usando o método do pseudopotencial PAW [59], tudo implementado no pacote computacional VASP [30]. Uma base de ondas planas foi usada, com ondas de energia inferior a $212 \mathrm{eV}$. Os pontos $\vec{k}$ da zona de Brillouin foram escolhidos de acordo com o esquema proposto por Monkhorst Pack [53], usando um mesh $5 \times 5 \times 1$ na qual a energia total estava convergida. A superfície (111), da célula romboédrico, de $\mathrm{Bi}_{2} \mathrm{Se}_{3}$ e $\mathrm{Bi}_{2} \mathrm{Te}_{3}$ foi investigada usando um método de fatias com uma camada de vácuo de pelo menos 20 Å. Usamos uma espessura de até 10 QLs, mantendo os parâmetros de rede experimentais.

\subsection{Resultados}

\subsubsection{Estabilidade energética}

Sabendo que isolantes topológicos desta família possuem uma possível célula unitária hexagonal alinhada na direção cartesiana [001] onde temos 3 QL inequivalentes A, B e 
C, cada uma rotacionada de $120^{\circ}$ em relação a anterior. A partir da geometria inicial ABCABCABC, geraram-se duas geometrias:

- Na primeira remove-se um QL, i.e, ABABCABC gerando o que chamamos de Intrinsic Stacking Fault (ISF).

- Na segunda adiciona-se uma QL, i.e, ABBCABCABC gerando o que chamamos de Extrinsic Stacking Fault (ESF).

Ambas podem ser vistas na figura 20. Para cada configuração obteve-se a distância

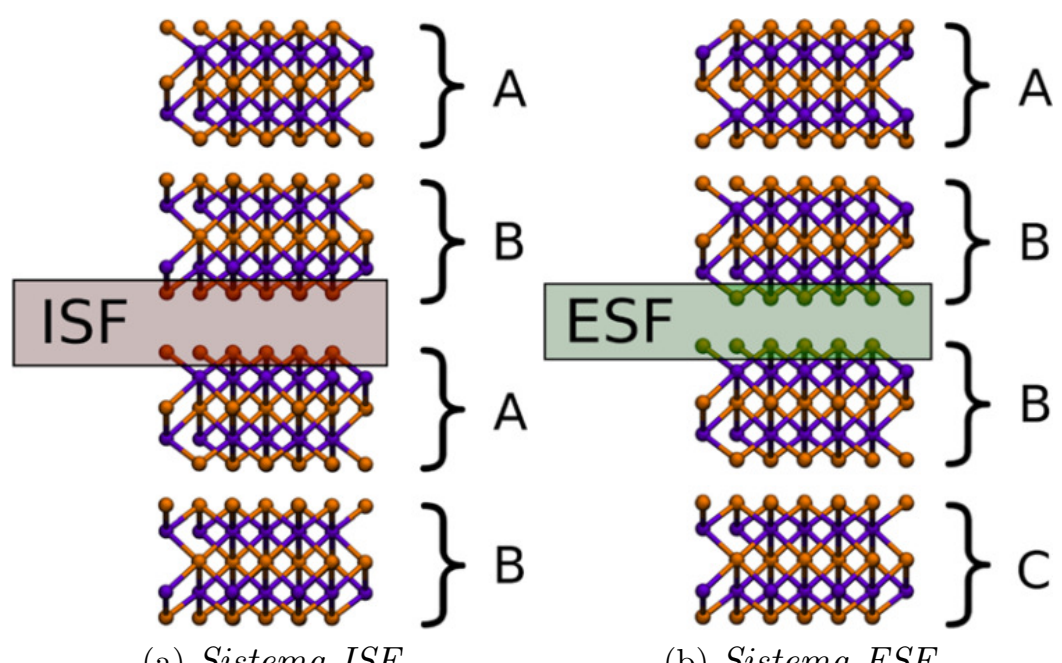

(a) Sistema ISF.

(b) Sistema ESF.

Figura 20: Modelos estruturais das falhas de empilhamento, à esquerda temos ISF e à direita temos ESF.

de equilíbrio via minimização de energia, onde todos os átomos foram mantidos fixos, e variou-se apenas a distância de Van Der Waals entre QLs, visto na figura 21. Nota-se que o alinhamento puro $(\mathrm{AB}, \mathrm{BC}$ ou $\mathrm{CA})$ representa a configuração mais estável com distância de equilíbrio igual a $2.15 \AA\left(\mathrm{Bi}_{2} \mathrm{Se}_{3}\right)$ e $2.56 \AA\left(\mathrm{Bi}_{2} \mathrm{Te}_{3}\right)$, ambos de acordo com resultados experimentais [91] e cálculos de primeiros princípios [84,92]. A distância vertical de equilíbrio e energias totais aumentam na presença da falha, indicando que a formação é endotérmica. Para ESF em $\mathrm{Bi}_{2} \mathrm{Se}_{3}$ e $\mathrm{Bi}_{2} \mathrm{Te}_{3}$ temos um aumento de energia total $(\Delta E)$ de 107 e $82 \mathrm{~mJ} / \mathrm{m}^{2}$ respectivamente. Enquanto que para ISF em $\mathrm{Bi}_{2} \mathrm{Se}_{3}$ e $\mathrm{Bi}_{2} \mathrm{Te}_{3}$ temos um aumento de $\Delta E$ de 212 e $196 \mathrm{~mJ} / \mathrm{m}^{2}$. As energias de formação das falhas de empilhamento 


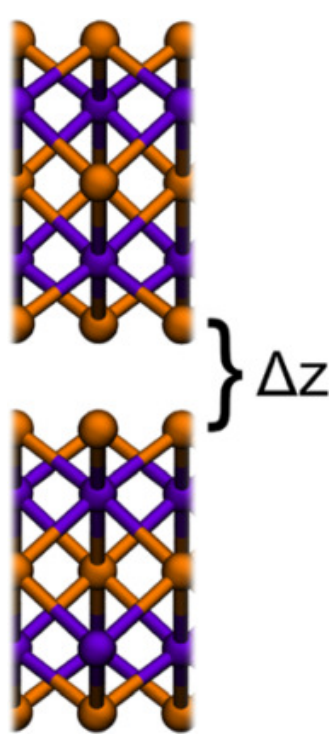

(a) Separação $\Delta z$.
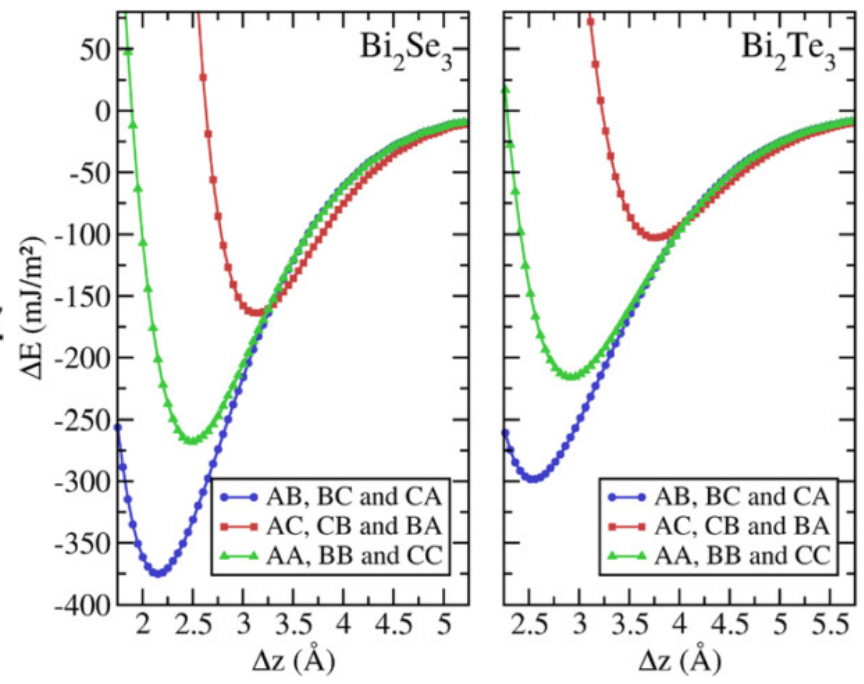

(b) Energia de ligação, em função de $\Delta z$.

Figura 21: (a) Representação da separação entre QLs, $\Delta z$. (b) Energia de ligação entre QLs, em função de $\Delta z$, para ISF e ESF em $\mathrm{Bi}_{2} \mathrm{Se}_{3}$ (esquerda) e $\mathrm{Bi}_{2} \mathrm{Te}_{3}$ (direita).

estão de acordo com a de outros materiais, onde temos Si 33 (ISF) e 26 (ESF) mJ/m² [93], Ni 187 (ISF) e 149 (ESF) mJ/m² e Rh 750 (ISF) e 291 (ESF) mJ/m².

Cada uma das configurações apresenta mínimos locais geométricos, onde $\Delta z$, vistos na tabela 2, é superior para ISF onde há alinhamento de átomos no eixo z. Os aumentos são de $12 \%$ para ESF em ambos os materiais e chegam a até $47 \%$ para ISF.

\begin{tabular}{lcc} 
Material & $\mathrm{Bi}_{2} \mathrm{Se}_{3}$ & $\mathrm{Bi}_{2} \mathrm{Te}_{3}$ \\
\hline$E S F$ & 2.40 & 2.91 \\
$I S F$ & 3.15 & 3.74
\end{tabular}

Tabela 2: Distâncias de $\Delta z$ em $\AA$, para cada configuração de Stacking Faults.

Observando que o alinhamento dos átomos ao longo do eixo z no ISF, proporciona um $\Delta z$ maior, concluímos que a falha de empilhamento enfraquece a interação de Van Der Waals entre QL, sendo comandada pela interação eletrostática iônica.

\subsubsection{Criação de estados metálicos em fase bulk}

A formação de falhas de empilhamento altera as propriedades eletrônicas do material. A intensidade de mudança é diferente para cada falha e para cada elemento da família 
destes materiais. Com a presença das falhas vimos que estados eletrônicos aparecem dentro do gap na fase bulk. No caso do sistema bulk de $\mathrm{Bi}_{2} \mathrm{Se}_{3}$ como visto na figura 22:

- A falha ESF proporciona apenas estados próximos a região de valencia $v_{1}$, que aparecem em torno do ponto $\Gamma$.

- A falha ISF, por outro lado, faz com que estados próximo a região de condução $c_{1}$ e outra próxima a região de valencia $v_{1}$, ambas nas intermediações do ponto $\Gamma$.

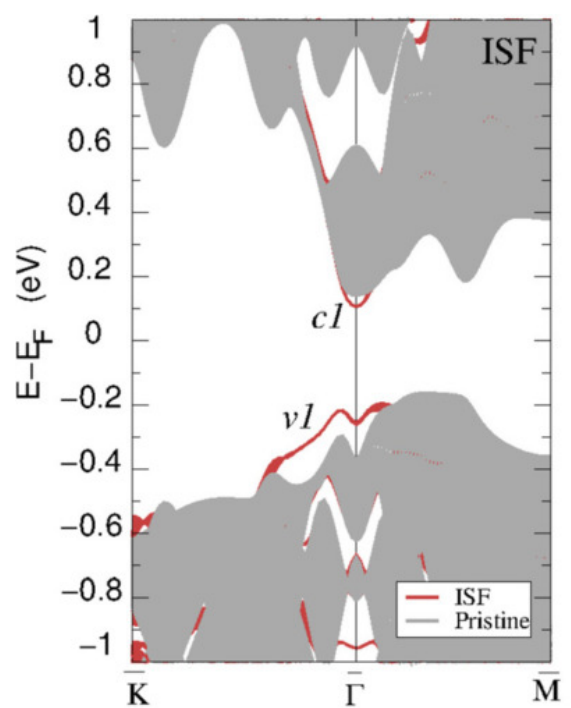

(a) Banda de bulk ISF.

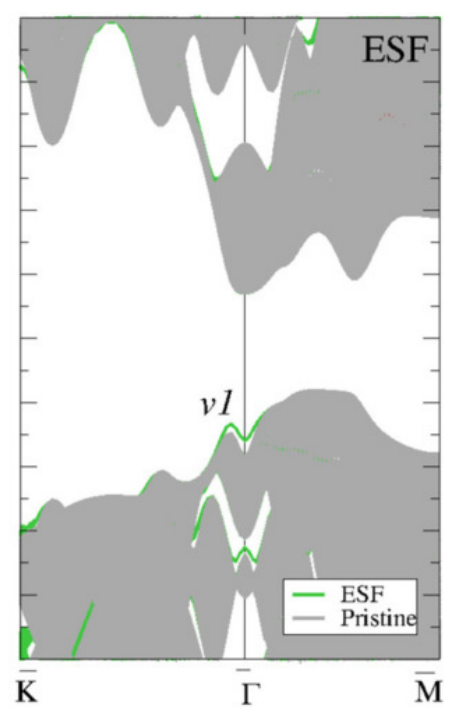

(b) Banda de bulk ESF.

Figura 22: Estrutura de banda eletrônica de $\mathrm{Bi}_{2} \mathrm{Se}_{3}$ em sua fase bulk com (a) um defeito ISF e (b) um ESF. Regiões em cinza representam projeções do bulk na ausência do defeito.

Agora para o caso de $\mathrm{Bi}_{2} \mathrm{Te}_{3}$ como visto na figura 23:

- Para ESF, o inverso do caso de $\mathrm{Bi}_{2} \mathrm{Se}_{3}$ ocorre onde apenas estados próximos à condução aparecem também em torno do ponto $\Gamma$.

- Para ISF, em grande contraste com os outros casos, estados metálicos surgem destruindo suas propriedades isolantes de bulk.

De fato é possível simular este efeito e gerar estados de borda apenas abrindo o espaçamento de Van der Waals, diminuindo a interação entre superfícies antes coladas. Para mostrar isso fizemos vários sistemas onde, variava-se a distância entre QLs, e plotamos as bandas vista na figura 24. Com isso, de fato, qualquer defeito nesta região, que não 


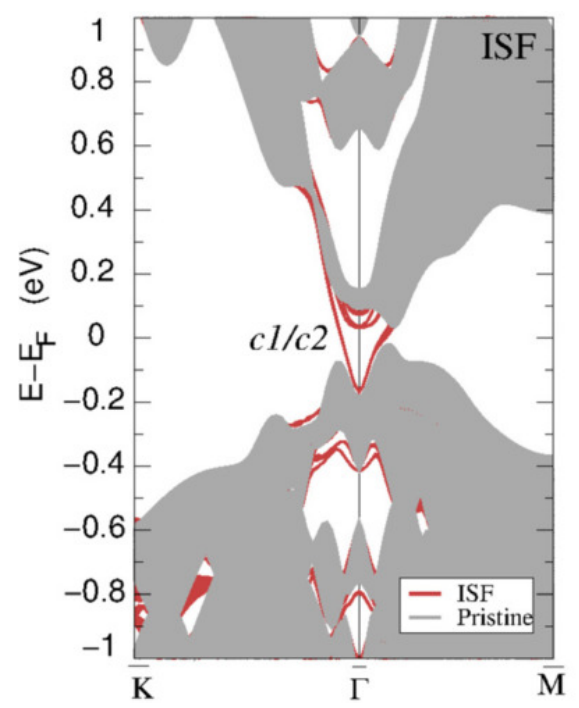

(a) Banda de bulk ISF.

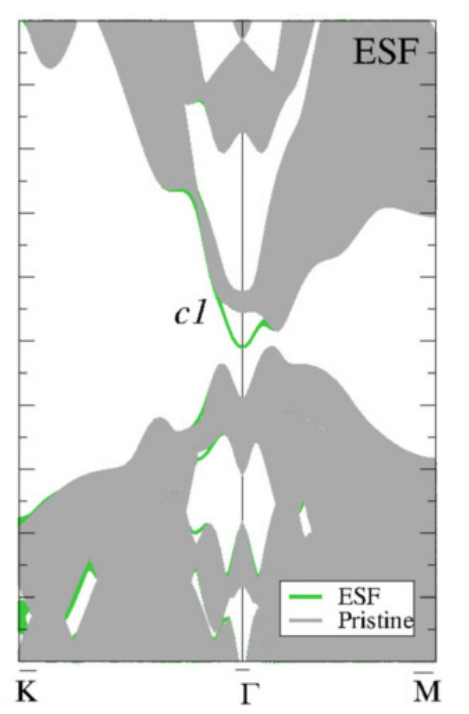

(b) Banda de bulk ESF.

Figura 23: O mesmo que a figura 22, para o caso do material $\mathrm{Bi}_{2} \mathrm{Te}_{3}$.

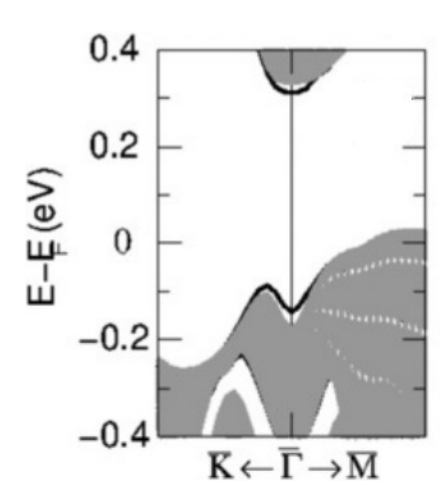

(a) $\delta z=2.50 \AA$.

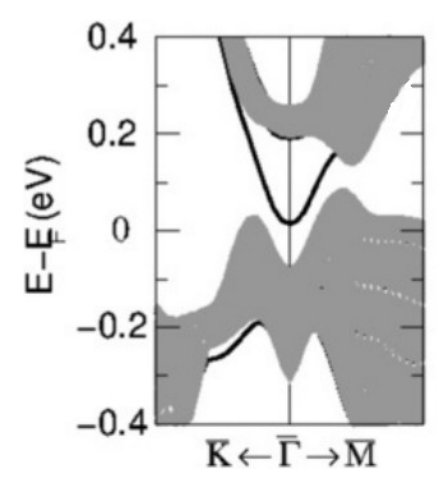

(e) $\delta z=2.91 \AA$.

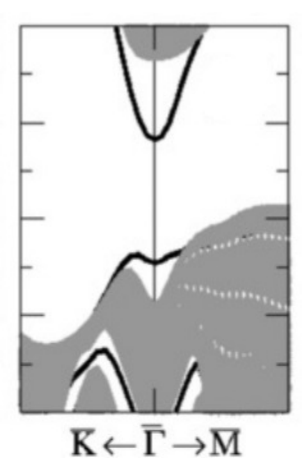

(b) $\delta z=3.15 \AA$.

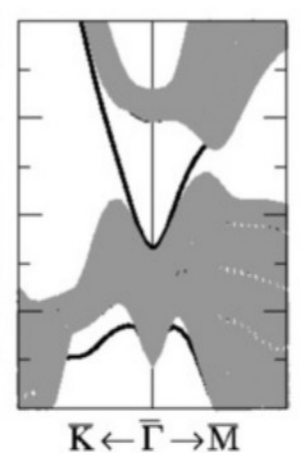

(f) $\delta z=3.26 \AA$.

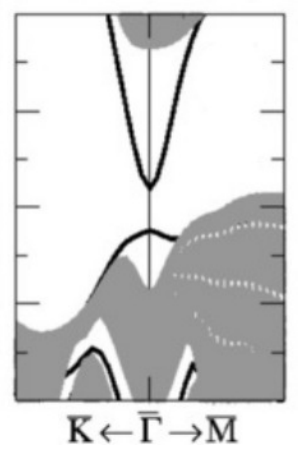

(c) $\delta z=4.15 \AA$.

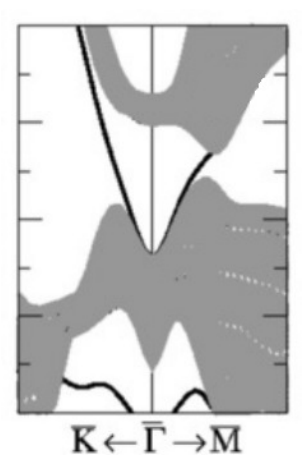

(g) $\delta z=3.76 \AA$.

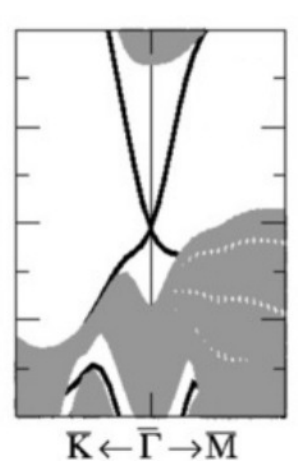

(d) $\delta z=7.15 \AA$.

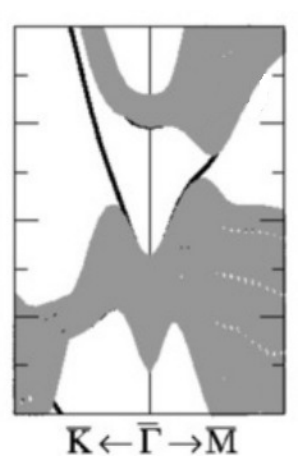

(h) $\delta z=7.56 \AA$.

Figura 24: Evolução dos estados em função da distância entre $Q L s$, $\delta z$ para $\mathrm{Bi}_{2} \mathrm{Se}_{3}$ : (a) 2.50 $\AA$, (b) $3.15 \AA$, (c) $4.15 \AA$, (d) $7.15 \AA$; e para $B i_{2} T e_{3}$ : (e) $2.91 \AA$, (f) $3.26 \AA$, (g) $3.76 \AA$, (h) $7.56 \AA$. Em cinza temos os estados provenientes do bulk pristino de cada sistema. 
interaja, mas aumente a distância entre camadas pode criar estados de borda. Na figura 24 vemos que variações da abertura entre as Qls pode levar a estados metálicos. A partir da análise feita percebemos que variações tão intensas como $\Delta z=3.56 \AA$ proporcionam o cone de Dirac em $\mathrm{Bi}_{2} \mathrm{Te}_{3}$. Para $\mathrm{Bi}_{2} \mathrm{Se}_{3}$ a abertura necessária para o surgimento do cone de Dirac é de $6.15 \AA$.

Em nossos resultados os cones de Dirac posicionam-se abaixo do topo da banda de valência, sendo $0.06 \mathrm{eV}$ para $\mathrm{Bi}_{2} \mathrm{Se}_{3}$ e $0.12 \mathrm{eV}$ para $\mathrm{Bi}_{2} \mathrm{Te}_{3}$, de acordo com outros cálculos téoricos [94]. Condutividade vinda do cone de Dirac pode ser camuflada pela condutividade remanescente de estados projetados da região do bulk [19].

\subsubsection{Corrente de spin em Superfície.}

Para $\mathrm{Bi}_{2} \mathrm{Se}_{3}$ estes defeitos de empilhamento perturbam fracamente os estados de superfície quando encontram-se enterrados no bulk. Um cenário diferente aparece quando as falhas ocorrem próximos à superfície de $\mathrm{Bi}_{2} \mathrm{Se}_{3}$ como visto na figura 25 (Note que temos aqui um sistema com 10 QLs).

Aqui vemos que um ESF promoveu um deslocamento vertical do cone de $\sim 50 \mathrm{meV}$ em relação ao caso pristino, e para ISF, tal deslocamento foi de $\sim 94 \mathrm{meV}$. Efeitos similares de quebra de degenerescência foram verificados em filmes finos de $\mathrm{Bi}_{2} \mathrm{Se}_{3}$ com aplicação de campo elétrico externo [94]. Isto mostra que falhas de empilhamento próximos à região de superfície podem isolar contribuições da condutividade vindas da região de bulk possibilitando o desenvolvimento de dispositivos baseados em spin. No caso de $\mathrm{Bi}_{2} \mathrm{Te}_{3}$ falhas próximas à superfície não alteram significativamente sua estrutura de bandas. 


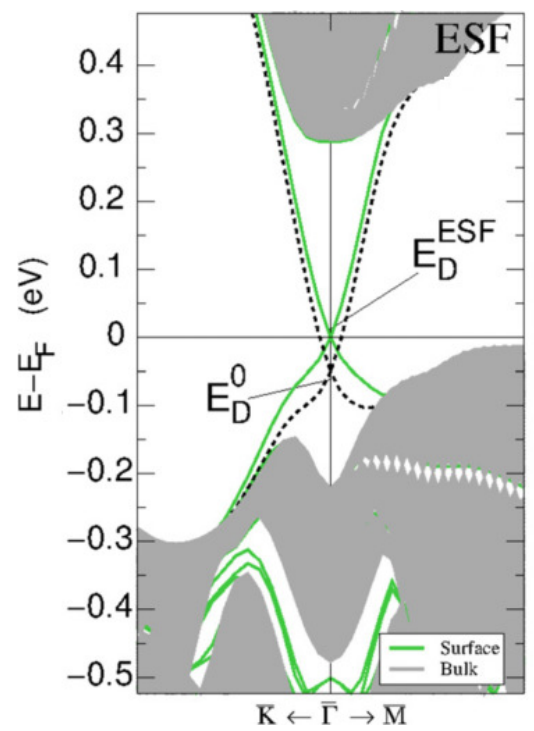

(a) Banda de superfície ISF.

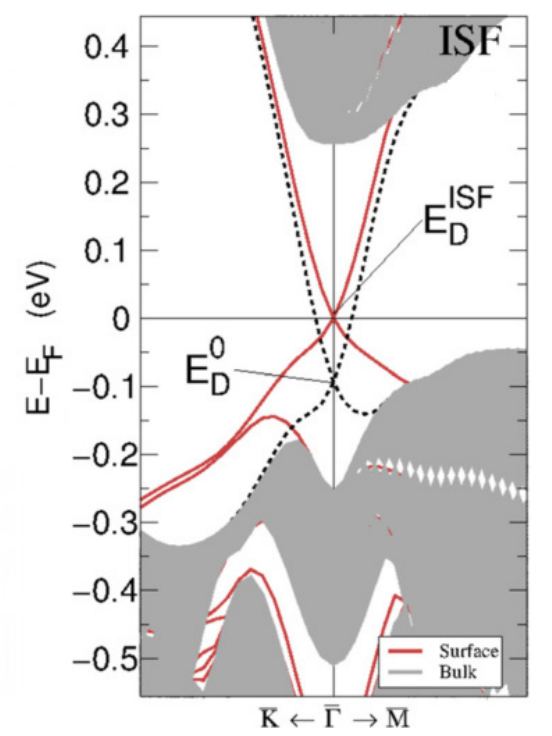

(b) Banda de superfície ESF.

Figura 25: Estrutura de banda eletrônica na presença de (a) ESF e (b) ISF em filmes finos de $\mathrm{Bi}_{2} \mathrm{Se}_{3}$. Linhas sólidas representam a estrutura eletrônica de banda da superfície (0001), na presença de SF. Linhas tracejadas representam a estrutura de banda do sistema pristino e regiões em cinza representam os estados de bulk projetados. $E_{D}^{E S F}\left(E_{D}^{I S F}\right)$ indicam a posição em energia do cone de Dirac na presença de ESF (ISF), e $E_{D}^{0}$ indica a posição em energia do cone de Dirac do caso pristino.

\subsection{Conclusão Parcial}

Com o auxílio de cálculos de primeiros princípios vimos que falhas de empilhamento podem gerar estados métalicos mesmo em fase bulk para o caso de $\mathrm{Bi}_{2} \mathrm{Te}_{3}$, enquanto que o caráter isolante é preservado em $\mathrm{Bi}_{2} \mathrm{Se}_{3}$. Quando está falha está próxima à superfície de filmes finos de $\mathrm{Bi}_{2} \mathrm{Se}_{3}$ vemos um deslocamento energético do cone de Dirac suprimindo canais de condutividade advindas da região de bulk, além de proporcionar um filtro de spin necessário para aplicação em spintrônica. Este trabalho foi publicado na revista Journal of Applied Physics [95]. Também são autores Leandro Seixas, Tomé Schmidt, Adalberto Fazzio e Roberto Miwa. 


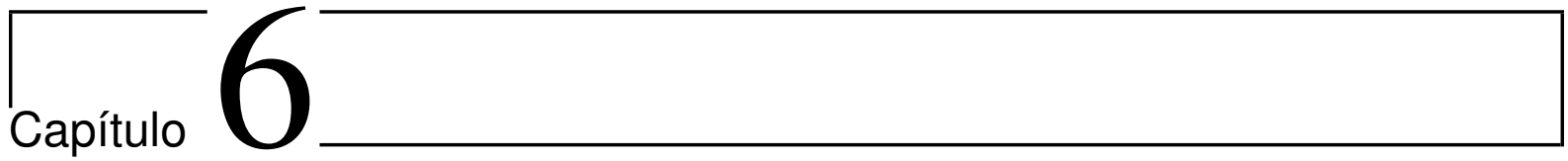

\section{Superfície do Isolante Topológico}

\section{$\mathrm{Bi}_{2} \mathrm{Se}_{3}(111)$, dopado com metais de transição: Uma análise de primeiros princípios}

Este capítulo representa o resultado principal da tese. Foi visto que, na superfície da célula romboédrico (111) do $\mathrm{Bi}_{2} \mathrm{Se}_{3}$, os estados topologicamente protegidos apresentam um único cone de Dirac, no centro da zona de Brillouin, enquanto que sua fase bulk apresenta um gap de energia (invertido no ponto $\Gamma$ ) $300 \mathrm{meV}$. Por estas razões $\mathrm{Bi}_{2} \mathrm{Se}_{3}$ é considerado um isolante topológico (TI - Topological Insulator) muito promisor para aplicações eletrônicas e em spintrônica $[17,76,84,96]$. Aplicações de TI dependem de processos de dopagem, como a incorporação de impurezas magnéticas, que assumirão o mesmo papel que um campo magnético, porém apenas localmente. Ou seja, haverá quebra da simetria de reversão temporal (TRS - Time Reversal Symmetry), levantando a degenerescência de Kramer no cone de Dirac, dando origem a férmions massivos, e permitindo processos de retroespalhamento.

A interação entre a impureza e os estados de superfície é um assunto ainda muito delicado e requer bastante atenção, dado que sua hibridização com a superfície dita as propriedades eletrônicas dos estados topológicos de superfície (TSSs - Topological Surface States). Grande esforço tem sido feito para entender tal interação. (i) Imagens de 
STM [97-100], e core-level spectroscopy [101], já foram utilizados para identificar a geometria dos átomos dopados na superfície do TI. Enquanto isso (ii) bandas de estrutura eletrônica dos TSS foram caracterizadas através de experimentos ARPES (Angle Resolved Photoemission Spectroscopy). De toda forma uma série de contradições existem entre (i) e (ii).

Por exemplo, gaps de energia no cone de Dirac (DP Dirac Point) foram verificados para as impurezas magnéticas Fe e Mn [100,102-104] que vão de 50 meV até 100 meV dependendo do sítio e concentração de impureza, enquanto que outros autores $[101,105]$ confirmam que o DP foi mantido sob deposição de impurezas magnéticas como o Gd e Fe nas superfícies de $\mathrm{Bi}_{2} \mathrm{Se}_{3}$. Além disso vários artigos [97, 98, 100, 106, 107], mostram uma discussão intensa em relação a configurações de equilíbrio de várias impurezas magnéticas em TIs.

Trabalhos teóricos, baseados em modelos de hamiltoniano efetivo [108], indicam que a quebra de degenerescência no ponto $\Gamma$ está associada com uma magnetização perpendicular ao plano. Outros grupos baseados em DFT apoiam que a perda de simetria de reversão temporal e subsequente abertura de um gap de energia exista na presença de impurezas magnéticas na superfície de um TI [109-113]. Por outro lado estudos teóricos baseados nas mesma aproximações mostram que magnetizações paralelas ao plano meramente deslocam o cone de Dirac do centro da zona de Brillouin $[114,115]$.

Neste capítulo fizemos uma análise sistemática do $\mathrm{TI} \mathrm{Bi}_{2} \mathrm{Se}_{3}(111)$ dopado com metais de transição (TM - Transition Metals). Através de cálculos de energia total apresentamos um mapa detalhado de estabilidade energética dos TM inseridos na superfície de $\mathrm{Bi}_{2} \mathrm{Se}_{3}$. Mudanças na densidade de carga da superfície dopada foram examinadas por cálculos de STM, e comparados com imagens de STM experimentais. Além de montar estruturas de bandas eletrônicas, para olhar a evolução do cone de Dirac sob a presença das impurezas magnéticas, propomos um modelo téorico que explica as várias magnetizações observadas, tanto perpendicular como paralela ao plano, e como isso está relacionado com a eletrônica de forma a sanar contradições de trabalhos citado acima. 


\subsection{Metodologia}

Cálculos foram feitos no âmbito de DFT [32,34], usando o método do pseudopotencial com PAW [59,60] para descrever as interações elétron íon. A função de troca e correlação foi calculada pela aproximação GGA-PBE [46], como implementado no código VASP [30], é sabido que o funcional escolhido é capaz de predizer a estrutura, porem subestima o tamanho do gap. Os orbitais de Kohn-Sham foram expandidas em uma base de ondas planas até um corte em energia de $270 \mathrm{eV}$. A zona de Brillouin foi escolhida por um conjunto de pontos $\vec{k}$ centrado no ponto $\Gamma$, usando um número de pontos até $2 \times 2 \times 1$ incluindo o ponto $\Gamma$, até que a energia total estivesse convergida. Vimos em capítulos anteriores a estrutura do material $\mathrm{Bi}_{2} \mathrm{Se}_{3}$, neste trabalho usamos uma supercélula com parâmetros estruturais hexagonais para simular a superfície, que no caso romboédrico seria $\mathrm{Bi}_{2} \mathrm{Se}_{3}(111)$. O método de slab ou fatias foi usado com uma camada de vácuo de $20 \AA$ para prevenir interações entre superfícies adjacentes. A célula unitária possui 4 quintuple layer (fatias com 5 camadas atômicas), de maneira a descrever corretamente o cone de Dirac [94] e uma periodicidade de superfície de $3 \times 3$ que, ao se dopar com apenas um único metal de transição, corresponderia a uma dopagem de aproximadamente $10 \%$.

As quatro primeiras camadas incluído o metal de transição (TM) são permitidas relaxar até forças atômicas atingirem valores menores que $10 \mathrm{meV} / \AA$. A energia de formação de $\mathrm{TM} / \mathrm{Bi}_{2} \mathrm{Se}_{3}(111)$ foi calculada de acordo com [116], visto na seguinte equação:

$$
E^{F}=E\left[\mathrm{TM} / \mathrm{Bi}_{2} \mathrm{Se}_{3}(111)\right]-E\left[\mathrm{Bi}_{2} \mathrm{Se}_{3}(111)\right]+n \mu_{\mathrm{Bi} / \mathrm{Te}}-\mu_{\mathrm{TM}},
$$

onde $\mathrm{E}\left[\mathrm{TM} / \mathrm{Bi}_{2} \mathrm{Se}_{3}(111)\right]$ e $\mathrm{E}\left[\mathrm{Bi}_{2} \mathrm{Se}_{3}(111)\right]$ são respectivamente as energias totais do slab com e sem a presença da impureza. O valor de $n$ pode ser 1 para geometrias substitucionais ou 0 para configurações intersticiais e adsorvidas, $\mu_{\mathrm{Bi} / \mathrm{Te}}$ e $\mu_{\mathrm{TM}}$ são energias totais por átomo de suas respectivas fases bulk mais estáveis. A precisão dos potenciais químicos calculados foram verificados através do cálculo de energias de coesão de suas fases mais estáveis como visto na tabela 3 e comparada com cálculos anteriores. O potencial químico de uma espécie é a derivada da energia livre com respeito ao número de partículas daquela 


\begin{tabular}{|c|c|c|c|c|}
\hline \multicolumn{4}{|c}{ Energias de Coesão } & Potencial químico \\
\hline & & & & \\
Átomos & Teórico [117] & $\operatorname{Exp}[118]$ & Trabalho & $\mu(\mathrm{eV})$ \\
\hline $\mathrm{Cr}$ & 3.80 & 4.10 & 4.03 & -9.517 \\
$\mathrm{Mn}$ & 3.73 & 2.92 & 3.73 & -8.898 \\
$\mathrm{Fe}$ & 4.78 & 4.28 & 4.87 & -8.314 \\
$\mathrm{Co}$ & 4.88 & 4.39 & 5.27 & -7.112 \\
$\mathrm{Ni}$ & 4.52 & 4.44 & 4.46 & -5.576 \\
$\mathrm{Bi}$ & - & 2.18 & 2.16 & -4.490 \\
$\mathrm{Se}$ & - & 2.46 & 2.58 & -3.503 \\
\hline
\end{tabular}

Tabela 3: As seguintes energias de coesão foram obtidas calculando a energia de bulk por átomo de suas fases mais estáveis e subtraindo pela energia do átomo isolado, onde em cada conta inclui-se correções de spin órbita. Para estes cálculos orbitais de Kohn-Sham foram expandidos em uma base de ondas planas com uma energia de até $270 \mathrm{eV}$.

espécie. Ela reflete a mudança em energia livre quando o número de partículas de uma espécie muda. Podemos escrevê-lo da seguinte maneira:

$$
\mu_{i}=\left(\frac{\partial H}{\partial N_{i}}\right)_{N_{j \neq i}, S, P},
$$

onde o potencial aqui é a derivada da entalpia $\mathrm{H}$, em relação ao número de espécie $N_{i}$, com entropia e pressão constantes. Cada espécie química na sua fase bulk mais estável possui seu potencial correspondente ao ambiente extremamente rico, ou seja, como estamos considerando, em todas as contas acima, $\mu_{\mathrm{Bi}}=\mu_{\mathrm{Bi}}^{\mathrm{bulk}}=\mu_{\mathrm{Bi}}^{\max }\left(\mu_{\mathrm{Se}}=\mu_{\mathrm{Se}}^{\mathrm{bulk}}=\mu_{\mathrm{Se}}^{\max }\right)$. Sabendo que a energia entalpia de formação é dada por:

$$
\Delta H_{\text {formação }}=2 \mu_{\mathrm{Bi}}^{\max }+3 \mu_{\mathrm{Se}}^{\max }-E_{\mathrm{Tot}_{-\mathrm{Bi}_{2} \mathrm{Se}_{3}},}
$$

onde $E_{\mathrm{Tot}_{-} \mathrm{Bi}_{2} \mathrm{Se}_{3}}$ é a energia total do sistema $\mathrm{Bi}_{2} \mathrm{Se}_{3}$ dada pelos cálculos de primeiros princípios, $\mu_{\mathrm{Bi}}^{\max }$ e $\mu_{\mathrm{Se}}^{\max }$ são os potenciais químicos de Bi e Se nos seus respectivos ambientes ricos, ou mais estáveis. Ao fazer a operação matemática $\mu_{\mathrm{Bi}}^{\max }-\frac{\Delta H_{\text {formação }}}{2}$ obtemos o seguinte:

$$
\begin{aligned}
\mu_{\mathrm{Bi}}^{\max }-\frac{\Delta H_{\text {formação }}}{2} & =\frac{2 \mu_{\mathrm{Bi}}^{\max }-2 \mu_{\mathrm{Bi}}^{\max }-3 \mu_{\mathrm{Se}}^{\max }+E_{\mathrm{Tot}-\mathrm{Bi}_{2} \mathrm{Se}_{3}}}{2}, \\
& =\frac{E_{\mathrm{Tot}_{-} \mathrm{Bi}_{2} \mathrm{Se}_{3}-3 \mu_{\mathrm{Se}}^{\max }}}{2}=\frac{2 \mu_{\mathrm{Bi}}^{\min }}{2}=\mu_{\mathrm{Bi}}^{\min },
\end{aligned}
$$

onde $E_{\mathrm{Tot}_{-} \mathrm{Bi}_{2} \mathrm{Se}_{3}}-3 \mu_{\mathrm{Se}}^{\max }=2 \mu_{\mathrm{Bi}}^{\min }$, pois se o Se está no seu ambiente máximo ou rico, logo Bi está no seu ambiente mínimo ou pobre. Assim com estre truque podemos captar a variação do potencial químico em seu respectivo ambiente na condição pobre. A análise é 
análoga para Se. As energias de formação para impurezas substitucionais foram obtidas em uma condição rica em Bi (rica em Se), onde para analisar as condições pobre em

$\mathrm{Bi}$ (pobre em Se), teríamos que usar $\mu_{\mathrm{Bi}}=\mu_{\mathrm{Bi}}^{\min }=\mu_{\mathrm{Bi}}^{\text {bulk }}-\Delta H_{\mathrm{Bi}_{2} \mathrm{Se}_{3}} / 2\left(\mu_{\mathrm{Se}}=\mu_{\mathrm{Se}}^{\min }=\right.$ $\left.\mu_{\mathrm{Se}}^{\text {bulk }}-\Delta H_{\mathrm{Bi}_{2} \mathrm{Se}_{3}} / 3\right)$. Nossa entalpia de formação calculada foi de $1.37 \mathrm{eV}$, muito próxima à medida experimentalmente de $1.50 \mathrm{eV}$ [119]. Cálculos de STM baseados no esquema de Tersoff-Hamann [120] (explicada no apêndice A), foram obtidos para altura constante de aproximadamente $2 \AA(1 \AA)$ do átomo mais acima para os casos dopados (pristino).

Usamos um esquema RGB (Red Green Blue) de cores, onde azul escuro representa a ausência de carga e a saturação de cor varia em cada sistema para observar as sutis variações da densidade de carga.

As bandas de estrutura eletrônica foram analisadas seguindo o método proposto por Park et al [77], considerando uma porcentagem de $10 \%$ para a função de onda de KohnSham projetada ora nas duas primeiras mono-camadas (ML - Mono-Layers) em cima do slab ora na própria impureza no sistema $\mathrm{TM} / \mathrm{Bi}_{2} \mathrm{Se}_{3}(111)$.

\subsection{Resultados}

Nesta seção iremos partir da descrição do caso pristino $\mathrm{Bi}_{2} \mathrm{Se}_{3}(111)$, para subsequentemente analisar as propriedades estruturais da dopagem de metais de transição em sua superfície. Feito um mapa energético das configurações mais estáveis, analisaremos as propriedades eletrônicas dos sistemas de menor energia, além de observar se houve preservação de natureza topológica.

\subsubsection{Caso Pristino $\mathrm{Bi}_{2} \mathrm{Se}_{3}(111)$}

Na figura 26 temos a estrutura da superfície do sistema pristino $\mathrm{Bi}_{2} \mathrm{Se}_{3}(111)$, no equilíbrio verificamos que não há reconstrução de superfície, ou seja a estrutura exibe uma periodicidade similar a 1x1. E os átomos mais acima $\mathrm{Se}(\mathrm{Bi})$ exibem um deslocamento para dentro (para fora) de 0.15 (0.03)Å. Neste cenário as ligações mais acima de Bi-Se são comprimidas de $0.10 \AA(2.97 \rightarrow 2.87 \AA)$. Nossos cálculos de LDOS, que 

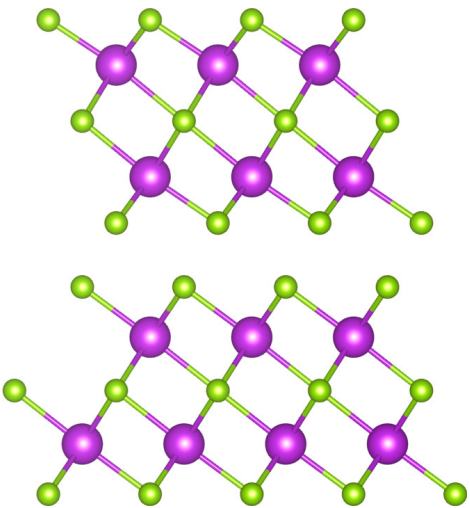

(a) Visão lateral

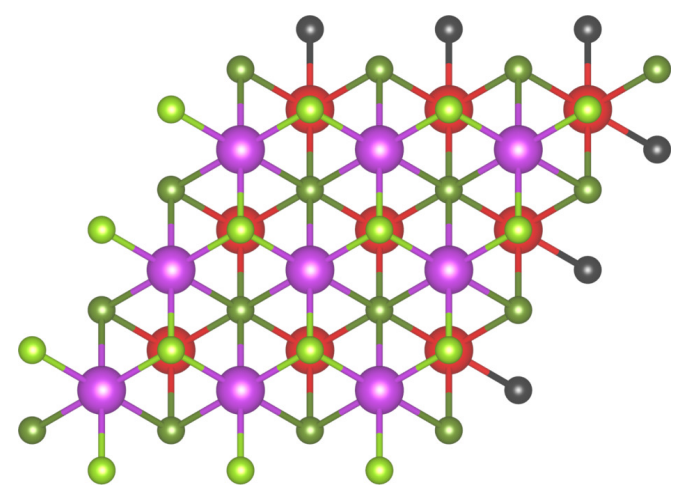

(b) Visão panorâmica

Figura 26: Modelo estrutural do sistema pristino $B i_{2} S e_{3}(111)$, em (a) temos as duas primeiras QLs com os dois tipos de átomos presentes, onde em verde e roxo temos Se e Bi, em (b) fizemos um código de cores para identificar as diferentes camadas, aonde temos verde claro, verde escuro, preto, violeta e vermelho, representando respectivamente a primeira, segunda e terceira camada de Se e a primeira e segunda camada de Bi.

na aproximação de Tersoff [120] chamaremos de imagens de STM, mostram formação de pontos brilhantes acima dos átomos mais acima de Se visto na figura 28a, confirmando a periodicidade 1x1 de acordo com resultados experimentais e téoricos [121,122]. Isolantes topológicos em contato com isolantes triviais promovem o aparecimento de estados metálicos. Para a superfície de $\mathrm{Bi}_{2} \mathrm{Se}_{3}(111)$ teremos estados topológicos de superfícies para vetores de onda paralelos ao plano, os TSSs. A figura 28b apresenta a estrutura de banda eletrônica pristina projetada onde a contribuição em cada ponto de banda foi selecionada considerando uma porcentagem crítica (maior que $10 \%$ ) da função de partícula única de Kohn-Sham. Ao lado apresentamos o mapeamento da zona recíproca. Aqui verifica-se a formação de um cone de Dirac localizado no ponto $\bar{\Gamma}$. Como a simetria de inversão espacial é quebrada na superfície, os TSS são spin separados pela interação do efeito Rashba-Bychkov [123]. Devemos lembrar que ainda temos a simetria de reversão temporal ou seja $E(\vec{k}, \uparrow)=E(-\vec{k}, \downarrow)$. De acordo com tal simetria e a separação de bandas devido ao efeito Rashba-Bychkov os vetores de polarização de spin $(\langle\vec{S}\rangle)$ de momento opostos $(\vec{k})$ devem ter sinais opostos. De forma que temos $\left\langle\vec{S}_{n}(\vec{k})\right\rangle=-\left\langle\vec{S}_{n}(-\vec{k})\right\rangle$, para uma dada banda n. Desta forma estas restrições fazem com que $(\langle\vec{S}\rangle)$ tenha uma textura helicoidal ao longo de um plano (perpendicular ao eixo cartesiano $\hat{k}_{z}$ ) da zona de 


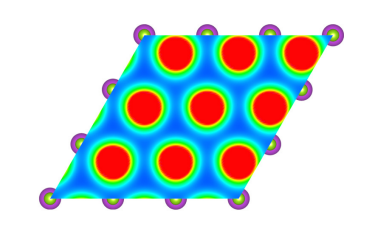

$-0.30 \mathrm{eV}$

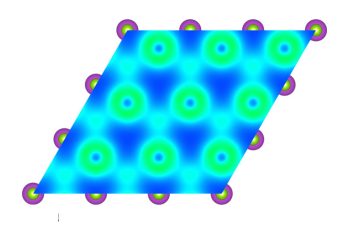

$+0.30 \mathrm{eV}$

(a) Imagens de STM.
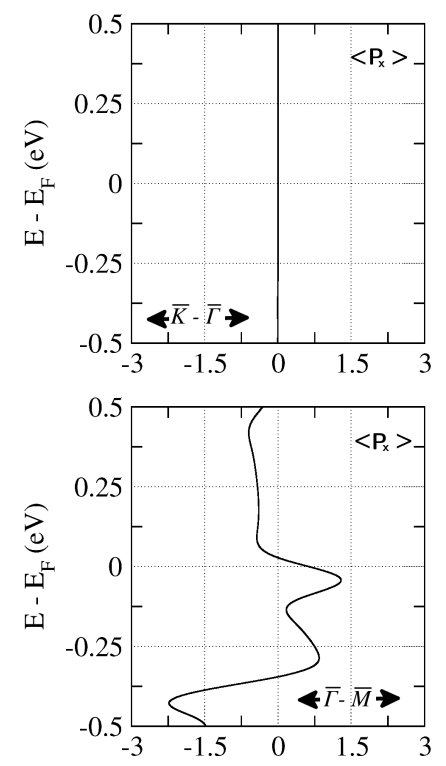

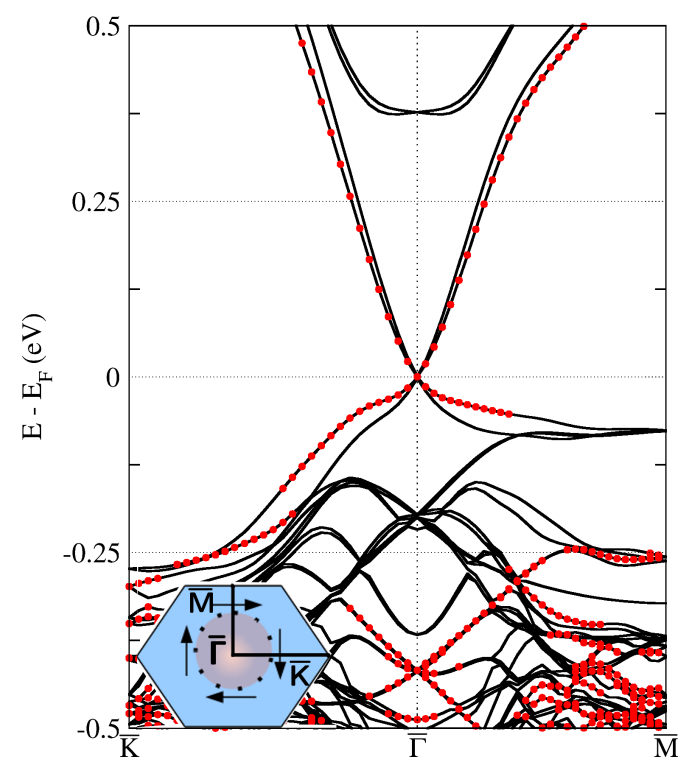

(b) Banda eletrônica do sistema pristino.
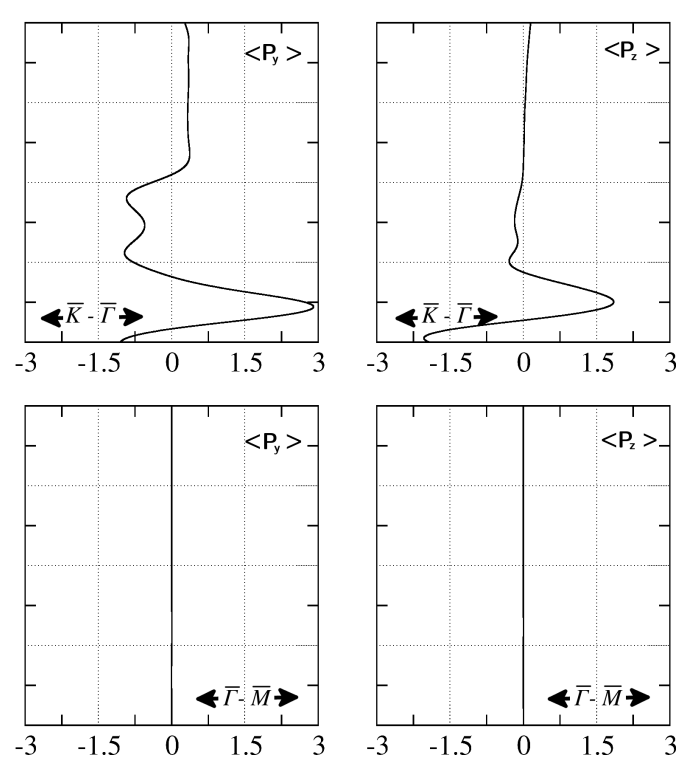

(c) SPDOS decomposta nas direções $\bar{\Gamma}-\bar{K}$ e $\bar{K}-\bar{M}$.

Figura 27: Propriedades eletrônicas do TI Bi $i_{2} S e_{3}(111)$, em (a) temos os STM em um intervalo de energia de $E_{F}= \pm 0.3 \mathrm{eV}$, em (b) linhas sólidas pretas indicam contribuição de todos os átomos para a banda do material, enquanto que em círculos vermelhos temos a contribuição para a banda vinda apenas das primeiras $2 M L$ as quais foram relaxadas. Em (c) temos a $P_{\alpha}(E)$ projetada apenas nas primeiras $2 M L$.

Brillouin [76,124]. Aqui o vetor polarização de spin pode ser escrito em termos de seus componentes cartesianos:

$$
\left\langle S_{n, \alpha}(\vec{k})\right\rangle=(\hbar / 2)\left\langle\phi_{n}(\vec{k})\left|\sigma_{\alpha}\right| \phi_{n}(\vec{k})\right\rangle \quad(\alpha=x, y, z)
$$

onde $\sigma_{\alpha}$ representa as matrizes de Pauli e $\phi_{n}(\vec{k})$ são autofunções de Kohn-Sham. A figura 28c apresenta a densidade de estados projetadas de spin que chamaremos de SPDOS (Spin 
Projected Density of States), onde para cada componente cartesiana de $(\langle\vec{S}\rangle)$ a SPDOS é definida pela equação (6.6).

$$
P_{\alpha}(E)=\sum_{n, \vec{k}}\left\langle S_{n, \alpha}(\vec{k})\right\rangle \delta\left(E-E_{n}(\vec{k})\right)
$$

De acordo com a textura heliciodal de spin perto do nível de Fermi na figura 28c, temos que:

- $\vec{k} \|$ à direção $\bar{\Gamma}-\bar{K}$ temos $P_{x}(E)=0$.

- $\vec{k} \|$ à direção $\bar{\Gamma}-\bar{M}$ temos $P_{y}(E)=0$.

- $\vec{k} \|$ à direção $\bar{\Gamma}-\bar{K}$ temos $P_{y}(E)<0(>0)$ para estados ocupados (vazios).

- $\vec{k} \|$ à direção $\bar{\Gamma}-\bar{M}$ temos $P_{x}(E)>0(<0)$ para estados ocupados (vazios).

Também podemos ver uma componente fora do plano $\left(P_{z}(E)\right)$ para vetores de onda paralelos à direção $\bar{\Gamma}-\bar{K}$ enquanto esta desaparece para $\bar{\Gamma}-\bar{M}$ o que esta relacionado a estados de fase bulk, pois não há contribuição nestes estados de átomos da superfície (pontos vermelhos na figura 28b).

\subsubsection{Propriedades estruturais de $\mathrm{TM} / \mathrm{Bi}_{2} \mathrm{Se}_{3}(111)$}

Agora que as propriedades estruturais e eletrônicas da superfície de $\mathrm{Bi}_{2} \mathrm{Se}_{3}(111)$ foram descritas e estão de acordos com a literatura tanto em trabalhos experimentais [76, 85], quanto em trabalhos teóricos [94], podemos começar a investigação de $\mathrm{Bi}_{2} \mathrm{Se}_{3}(111)$ dopados em sua superfície com metais de transição TM (Transition Metals). Aqui iremos investigar os seguintes TMs: Cr, Mn, Fe, Co e Ni, embutidos na primeira QL de $\mathrm{Bi}_{2} \mathrm{Se}_{3}(111)$. Como mostrado na figura 28 consideramos um grande número de configurações possíveis (substitucionais, intersticiais e adsorvidos). As configurações mais prováveis foram baseadas em cálculos de energia de formação apresentada na tabela 4. Muitos estudos foram feitos em relação à incorporação destes metais de transição em TI, porém muitos resultados são contraditórios. Falaremos de cada caso na sequência. A dopagem com $\mathrm{Fe}, \mathrm{Fe} / \mathrm{Bi}_{2} \mathrm{Se}_{3}(111)$ tem uma preferência energética para a configuração substitucional em 


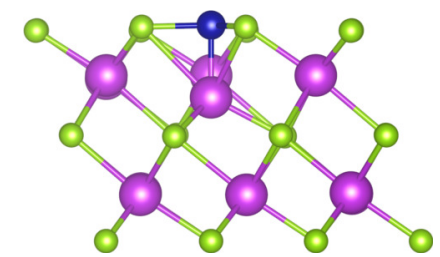

(a) Bi-ads.

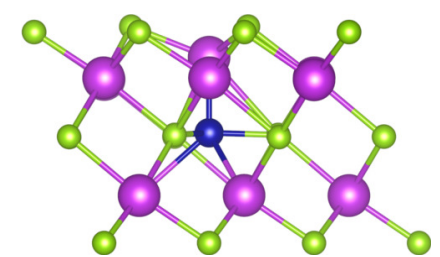

(c) Bi-int.

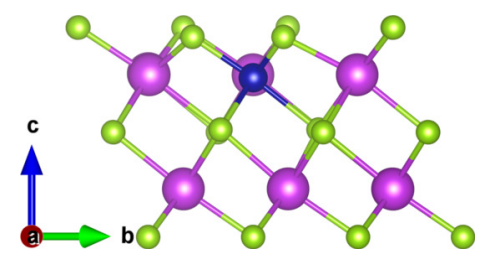

(e) Bi-sub.

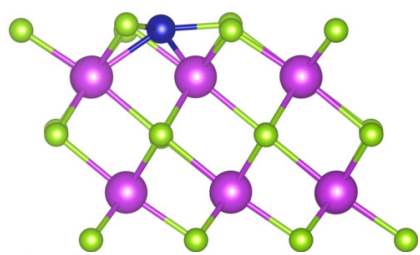

(b) $S e-a d s$.

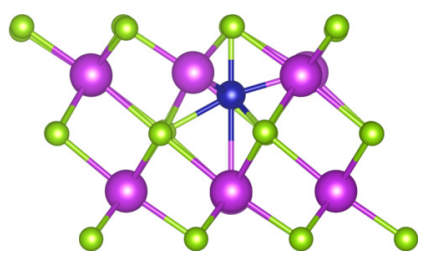

(d) Se-int.

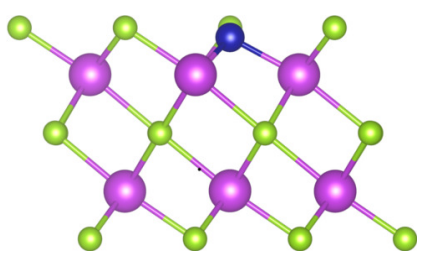

(f) $S e-s u b$.

Figura 28: Modelos estruturais dos sistemas $T M / B i_{2} S_{3}(111)$ pós relaxação. Note que este é um caso modelo de Co, porém os outros TM seguem qualitativamente os mesmos deslocamentos, apenas com valores diferentes que podem ser vistos nas tabelas 5 e 6 .

sítio de Bi aonde $E^{F}=1.05 \mathrm{eV}$, na condição rica em Bi, enquanto que na condição pobre em Bi esta configuração é ainda mais provável com $E^{F}=0.37 \mathrm{eV}$. Este átomo de $\mathrm{Fe}$ tem número de coordenação igual a 6 formando 3 ligações Fe-Se com os átomos de Se da primeira camada e outras 3 ligações Fe-Se com os átomos Se da terceira camada, como mostrado nas figuras 29a e 29b. As geometrias de equilíbrio estão descritas na tabela 6 . Nota-se uma geometria octaédrica local em torno da impureza, isso acarretará mudanças eletrônicas que veremos na próxima seção. Na geometria de equilíbrio, achamos ligações Fe-Se de $2.54 \AA$ com átomos próximos à superfície e $2.82 \AA$ com átomos mais internos. A energia de formação de Fe substitucional em Bi está de acordo com estudos recentes de TMs em $\mathrm{Bi}_{2} \mathrm{Se}_{3}[107,125]$ e $\mathrm{Bi}_{2} \mathrm{Te}_{3}$ dopados com Fe [126]. A configuração Bi-int vista em 29c e 29d é a segunda mais provável com $E^{F}=1.17 \mathrm{eV}$. Neste ambiente o Fe tem coordenação 7 formando 3 ligações Fe-Se de 2.40 A, 3 ligações Fe-Bi de 3.26 A e uma única ligação Fe-Bi paralela à direção (111) de $2.63 \AA$, de forma que este último átomo de Bi da segunda camada exibe um deslocamento vertical para cima de $0.89 \AA$ A. As distâncias h se 


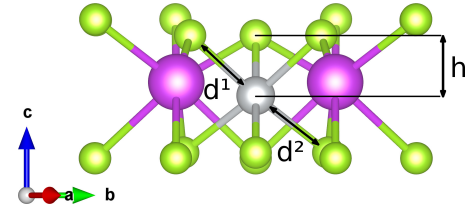

(a) Visão lateral de Bi-sub.

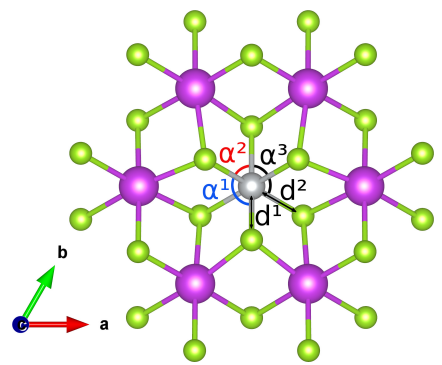

(b) Visão panorâmica de Bi-sub.

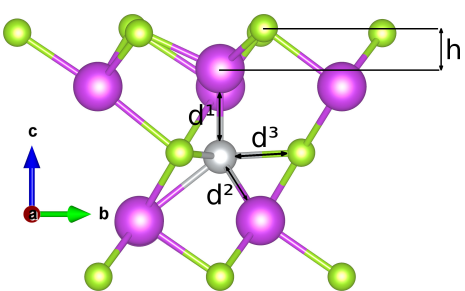

(c) Visão lateral de Bi-int.

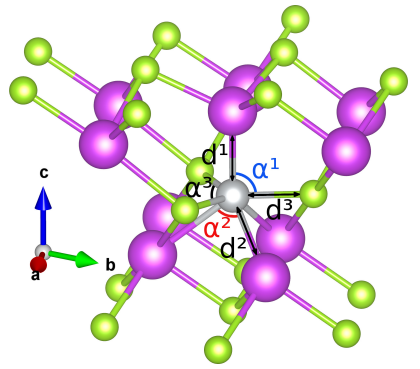

(d) Visão em perspectiva de Bi-int.

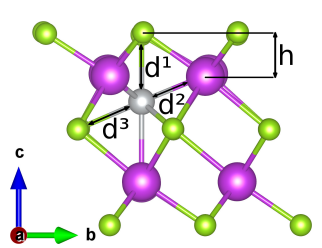

(e) Visão lateral de Se-int.

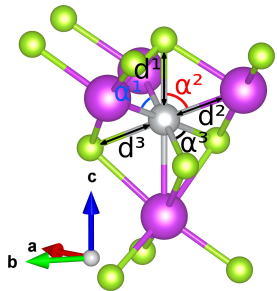

(f) Visão em perspectiva de Se-int.

Figura 29: Modelos estruturais dos casos Bi-sub, Bi-int e Se-int com os respectivos parâmetros que descrevem o sistema cujos valores estão descritos nas tabelas 5 e 6 .

\begin{tabular}{lccccc}
\hline \hline & & & & & \\
Configuração & Cr & Mn & Fe & Co & Ni \\
\hline & & & & & \\
Bi-sub & -0.10 & 0.04 & 1.05 & 1.69 & 1.33 \\
Se-sub & 3.34 & 2.96 & 3.58 & 3.44 & 2.33 \\
Bi-int & 1.14 & 0.46 & 1.17 & 1.14 & 0.63 \\
Se-int & 2.09 & 0.88 & 1.40 & 1.43 & 0.85 \\
Bi-ads & 1.36 & 0.74 & 1.28 & 1.30 & 0.75 \\
Se-ads & 2.49 & 0.87 & 1.40 & 1.35 & 0.68 \\
\hline
\end{tabular}

Tabela 4: Energias de formação (em eV) de dopagens substitucionais (Bi-sub em condição rica em Bi e Se-sub em condição rica em Se), intersticiais (int) e adsorvidas (ads) no $\mathrm{TI} \mathrm{Bi}_{2} \mathrm{Se}_{3}$.

reduzem de $1.59 \AA$ para o caso pristino para $0.92 \AA$. As geometrias de equilíbrio do caso intersticial estão detalhadas nas figuras 29c e 29d e resumidas na tabela 5. Recentemente duas geometrias (metaestáveis) foram consideradas boas candidatas para o Fe adsorvido na superfície de $\mathrm{Bi}_{2} \mathrm{Se}_{3}(111)$ as duas são Bi-ads e Se-ads detalhadas nas figuras 30a até 30d. Porém, não houve resultados conclusivos na literatura. Na ref [114] autores obtiveram uma pequena preferência energética de $(70 \mathrm{meV})$ para a configuração Se-ads, em contraste com outro grupo [109], que usando um método similar obtêm uma preferência 


\begin{tabular}{cccccccccccccc}
\hline \hline $\mathrm{TM}$ & $d^{1}$ & $d^{2}$ & $d^{3}$ & $\alpha^{1}$ & $\alpha^{2}$ & $\alpha^{3}$ & $\mathrm{~h}$ & & $d^{1}$ & $d^{2}$ & $\alpha^{1}$ & $\mathrm{~h}$ \\
\hline Bi-int & & & & & & & \multicolumn{3}{c}{ Bi-ads } & & & & \\
$\mathrm{Fe}$ & 2.63 & 3.26 & 2.40 & 93.40 & 79.18 & 119.65 & 0.92 & & 2.41 & 2.52 & 119.57 & 0.28 \\
$\mathrm{Mn}$ & 2.82 & 3.80 & 2.52 & 111.30 & 66.48 & 107.58 & 0.10 & & 2.48 & 2.68 & 118.79 & 0.27 \\
$\mathrm{Cr}$ & 2.82 & 3.61 & 2.50 & 105.71 & 70.49 & 112.95 & 0.31 & & 2.53 & 2.87 & 109.25 & 0.85 \\
$\mathrm{Co}$ & 2.57 & 3.20 & 2.36 & 91.72 & 80.66 & 119.91 & 1.04 & & 2.37 & 2.46 & 119.95 & 0.06 \\
$\mathrm{Ni}$ & 2.59 & 3.03 & 2.39 & 87.11 & 85.73 & 119.75 & 1.19 & & 2.35 & 2.42 & 119.98 & 0.03 \\
& & & & & & & & & & & & \\
$\mathrm{Se}-\mathrm{int}$ & & & & & & & & Se-ads & & & & \\
$\mathrm{Fe}$ & 2.24 & 2.68 & 3.55 & 116.30 & 78.76 & 93.69 & 1.72 & & 2.43 & 2.84 & 119.40 & -0.19 \\
$\mathrm{Mn}$ & 2.43 & 2.88 & 3.21 & 107.53 & 68.65 & 103.77 & 1.38 & & 2.44 & 3.12 & 119.46 & 0.18 \\
$\mathrm{Cr}$ & 2.20 & 2.74 & 3.73 & 118.24 & 82.32 & 90.64 & 1.83 & & 2.39 & 2.91 & 118.48 & -0.29 \\
$\mathrm{Co}$ & 2.32 & 2.71 & 3.35 & 112.37 & 73.61 & 98.77 & 1.55 & & 2.37 & 2.87 & 119.57 & -0.17 \\
$\mathrm{Ni}$ & 2.50 & 2.73 & 2.55 & 106.40 & 67.61 & 105.34 & 1.46 & & 2.38 & 2.82 & 119.42 & -0.18 \\
\hline
\end{tabular}

Tabela 5: Geometrias de equilíbrio das impurezas intersticiais e adsorvidas, onde distâncias atômicas ( $d$ e h) estão em $\AA$ e ângulos de ligações químicas ( $\alpha$ ) estão em graus.

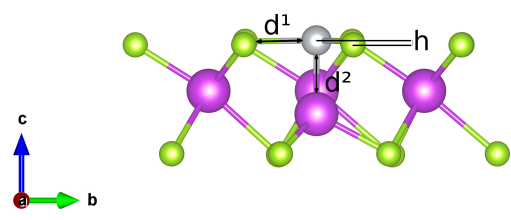

(a) Visão lateral de Bi-ads.

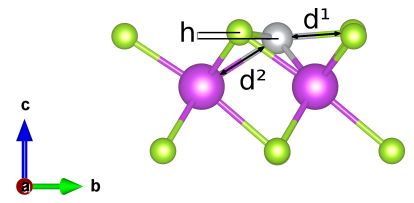

(c) Visão lateral de Seads.

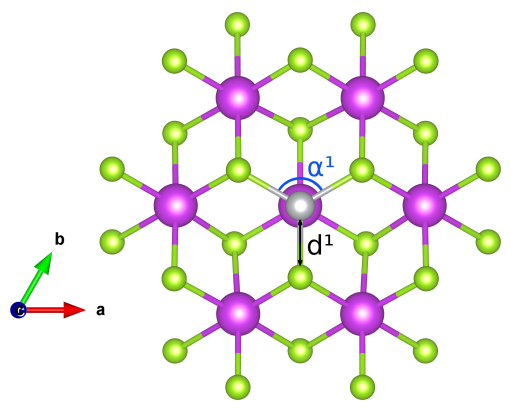

(b) Visão panorâmica de Biads.

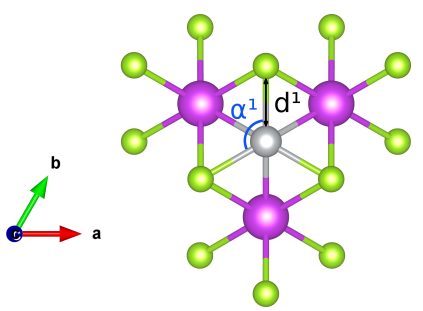

(d) Visão panorâmica de Se-ads.

Figura 30: Detalhes das estruturas adsorvidas pelos metais de transição e seus respectivos parâmetros que descrevem o sistema cujos valores estão descritos nas tabelas 5 .

de $75 \mathrm{meV}$ para a configuração Bi-ads. O último resultado está qualitativamente de acordo com nossas descobertas, Bi-ads é mais provável que Se-ads por $120 \mathrm{meV}$, como visto na tabela 4. Estudos de espectroscopia de níveis de caroço permitem uma melhor compreensão dos possíveis cenários desta impureza em $\mathrm{Bi}_{2} \mathrm{Se}_{3}$ [101]. Os autores propõem dois tipos de ambientes em função da temperatura de deposição. Eles reportam um shift 


\begin{tabular}{ccccccc}
\hline \hline & & & & & & \\
$\mathrm{TM}$ & $d^{1}$ & $d^{2}$ & $\alpha^{1}$ & $\alpha^{2}$ & $\alpha^{3}$ & $\mathrm{~h}$ \\
\hline Pristine & 2.87 & 3.10 & 92.32 & 91.80 & 83.76 & 1.59 \\
$\mathrm{Fe}$ & 2.54 & 2.82 & 90.90 & 91.41 & 86.19 & 1.44 \\
$\mathrm{Mn}$ & 2.60 & 2.70 & 86.57 & 91.78 & 89.82 & 1.59 \\
$\mathrm{Cr}$ & 2.54 & 2.65 & 86.17 & 91.54 & 90.66 & 1.56 \\
$\mathrm{Co}$ & 2.49 & 2.84 & 92.08 & 91.03 & 85.69 & 1.39 \\
$\mathrm{Ni}$ & 2.50 & 2.61 & 85.36 & 91.51 & 91.47 & 1.55 \\
\hline
\end{tabular}

Tabela 6: Geometrias de equilíbrio das impurezas substitucionais, onde distâncias atômicas (d e h) estão em $\AA$ e ângulos de ligações químicas $(\alpha)$ estão em graus.

do nível do caroço de Bi-TM(5d) atribuído à interação química entre Fe adsorvido e a superfície $\mathrm{Bi}_{2} \mathrm{Se}_{3}(111)$. O shift é mais intenso quando $\mathrm{Fe}$ é depositado a temperatura ambiente, e decresce $20 \%$ para baixas temperaturas. A presença de diferentes ambientes é identificada pela formação de ligações químicas de Fe-Bi e Fe-Se. Nossos resultados indicam a presença de tais ligações tanto para Bi-ads quanto para Bi-int. Nesta última verificamos que a interação $\mathrm{Fe} \longleftrightarrow \mathrm{Bi}$ foi fortificada comparada à primeira geometria Bi-ads. De qualquer forma podemos inferir o seguinte cenário para $\mathrm{Fe} / \mathrm{Bi}_{2} \mathrm{Se}_{3}(111)$ :

- À baixas temperaturas o Fe permanecerá em cima da superfície ou seja na configuração Bi-ads.

- Ao aumentar a temperatura, permitindo a segregação dos átomos de impureza, podemos ter a presença de Fe em sítios intersticiais de Bi.

Porém para altas temperaturas de deposição a formação das configurações substitucionais que são mais estáveis é esperada, neste caso a interação Fe-Bi é enfraquecida. Na geometria adsorvida de equilíbrio os átomos de Fe permanecem no mesmo plano que o Se mais acima com ligações Fe-Se de $2.41 \AA$ e $2.43 \AA$ para as geometrias Bi-ads e Se-ads respectivamente onde tais estruturas locais apresentam uma simetria $C_{3 v}$. Essas diferentes geometrias para a impureza de $\mathrm{Fe}$ em $\mathrm{Bi}_{2} \mathrm{Se}_{3}(111)$ proporcionam diferentes possibilidades para a distribuição da densidade de carga, e isso acarreta diferentes formatos para as imagens de STM. Nas figuras 31b e 31c apresentamos tais imagens para os casos de 
Bi-sub em um intervalo de energia de $0.3 \mathrm{eV}$ em relação ao nível de Fermi $\left(E_{F}= \pm 0.3\right.$ eV). Como vimos que as ligações de Fe-Se são menores que as de Bi-Se, os átomos de Se da primeira camada amarrados à impureza, se movem para baixo de $0.28 \AA$ em relação aos outros átomos Se da superfície mais afastados. Tal mudança estrutural pode ser identificada claramente nas imagens simuladas de STM. Verificamos a formação de uma figura triangular em cima dos átomos da primeira camada de Se logo acima da impureza. É importante notar que para os estados ocupados $31 \mathrm{~b}$ esta forma triangular é mais brilhante, mesmo dado o deslocamente para baixo dos átomos de Se, indicando um aumento na densidade de estados ocupados dentro da faixa entre $E_{F}$ e -0.3 eV na superfície em torno da impureza. Em contraste temos uma região triangular escura para os estados desocupados 31c. Nossos resultados se comparam muito bem com os obtidos por outras análises em $\mathrm{Fe} / \mathrm{Bi}_{2} \mathrm{Te}_{3}(111)$ [126]. Outras simulações mostram que as configurações Bi-int e Bi-ads apresentam diferentes formatos para a densidade de carga. No primeiro observamos um formato triangular simétrico (diferente dos anteriores) acima dos 3 átomos de Se da superfície visto em 31d e 31e, além disso como descrito anteriormente o Fe empurra o Bi da segunda camada para cima. Finalmente nas figuras 31f e $31 \mathrm{~g}$ temos os cálculos de STM para a geometria Bi-ads. Esta configuração pode ser identificada pela formação de um ponto brilhante central em cima da impureza, porém diferentemente das outras sua imagem é assimétrica devido à preferência energética de uma magnetização no plano $\langle\vec{S}\rangle$ que veremos na próxima seção. Nossas energias de formação mostram resultados similares para $\mathrm{Mn} / \mathrm{Bi}_{2} \mathrm{Se}_{3}(111)$ e $\mathrm{Cr} / \mathrm{Bi}_{2} \mathrm{Se}_{3}(111)$. Para ambos a geometria mais estável é a substitucional no sítio de Bi, seguido do caso intersticial no sítio de Bi. De fato dados experimentais comprovam tanto a formação da geometria $\mathrm{Mn} / \mathrm{Bi}_{2} \mathrm{Se}_{3}(111)$ no sítio substitucional de $\mathrm{Bi}[100,104]$ quanto na geometria $\mathrm{Mn} / \mathrm{Bi}_{2} \mathrm{Te}_{3}(111)$ no mesmo sítio [115]. Neste caso o Mn tem coordenação 6 com 3 ligações de $2.60 \AA$ e outras 3 de $2.70 \AA$ com os átomos de Se da primeira e terceira camadas respectivamente, como indicados nas figura 29a e 29b. Dado que tais ligações são menores, os átomos da primeira camada tem um deslocamento vertical para dentro de 0.31 A efeito observável nas imagens de STM. 


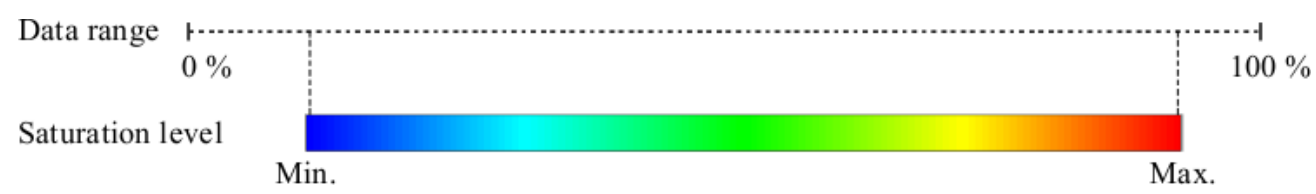

(a) Gradiente de cor no esquema $R-G-B$.

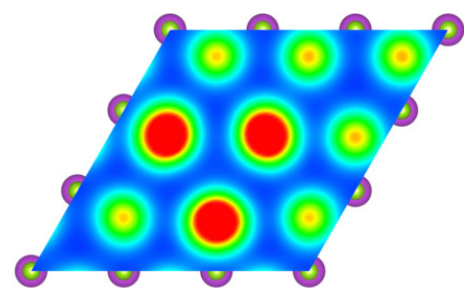

(b) STM est ocu Fe-Bi-sub.

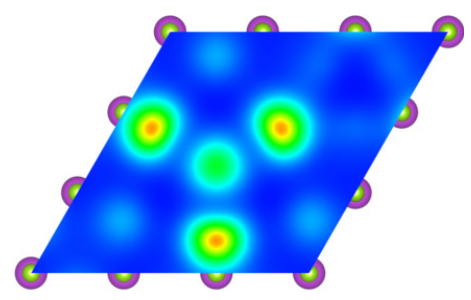

(d) STM est ocu Fe-Bi-int.

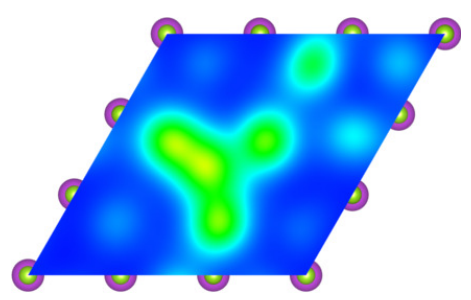

(f) STM est ocu Fe-Bi-ads.

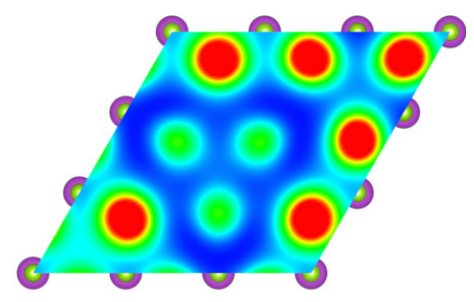

(h) STM est ocu $M n-B i-s u b$.

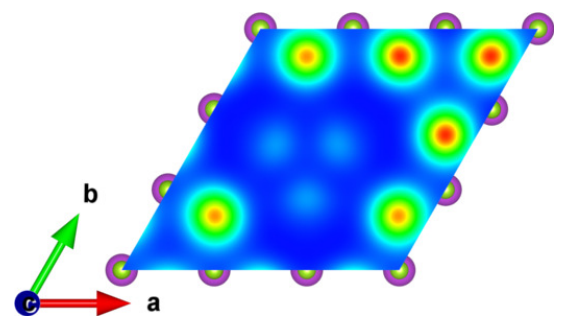

(j) STM est ocu $\mathrm{Cr}$-Bi-sub.

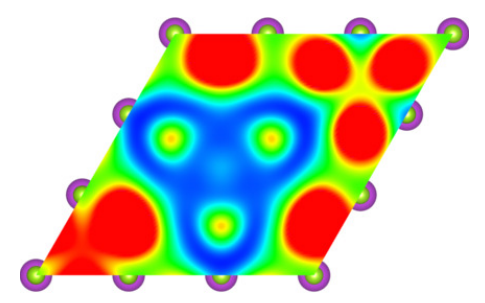

(c) STM est vaz Fe-Bi-sub.

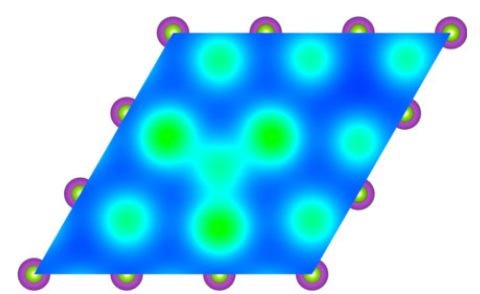

(e) STM est vaz Fe-Bi-int.

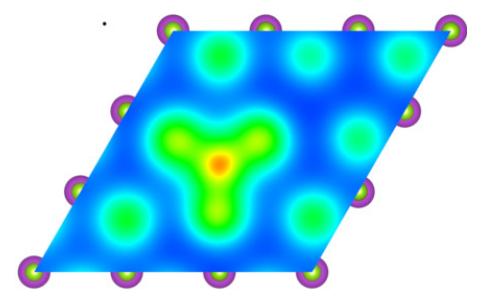

(g) STM est vaz Fe-Bi-ads.

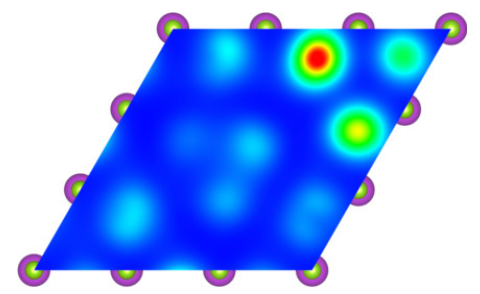

(i) STM est vaz $M n-B i-s u b$.

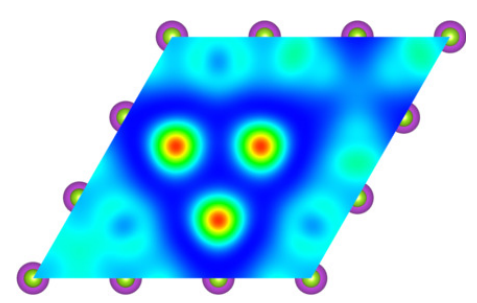

(k) STM est vaz $C r-B i-s u b$.

Figura 31: Imagens simuladas de STM para estados ocupados e vazios dentro de um intervalo de energia de $E_{F}= \pm 0.30 \mathrm{eV}$. 
Nas simulações de STM nota-se um brilho triangular que, diferentemente do caso do Fe, é menos intenso. O Cr sub-Bi é o único caso cujo processo de formação é exotérmico, onde $E_{F}=-0.10 \mathrm{eV}$, mesmo na condição rica ou pobre em Bi. Este efeito é observado teoricamente [125], além de ser experimentalmente possível [106,107]. Na geometria de equilíbrio, como nos outros casos temos uma simetria octaédrica local, o Cr liga-se aos Se da primeira e terceira camada, com distâncias de ligações de $2.54 \AA$ e $2.65 \AA$ respectivamente. A imagem de STM assemelha-se à do Mn como visto nas figuras $31 \mathrm{j}$ e $31 \mathrm{k}$ onde recupera-se a informação dos átomos de Se da primeira camada deslocando-se verticalmente para dentro de $0.35 \AA$. Por último vamos analisar os casos $\mathrm{Co} / \mathrm{Bi}_{2} \mathrm{Se}_{3}(111) \mathrm{e}$ $\mathrm{Ni} / \mathrm{Bi}_{2} \mathrm{Se}_{3}(111)$. Estes casos são dependentes do processo de formação. Na condição rica em Bi a energia de formação dos caso sub em Bi são improváveis sendo $E^{F}=1.69 \mathrm{eV}$ e $1.33 \mathrm{eV}$ para $\mathrm{Co}_{\mathrm{Bi}}$ e $\mathrm{Ni}_{\mathrm{Bi}}$. Porém na condição pobre em Bi o panorama energético muda, tendo $E^{F}=1.00 \mathrm{eV}$ e $0.65 \mathrm{eV}$ para $\mathrm{Co}_{\mathrm{Bi}}$ e $\mathrm{Ni}_{\mathrm{Bi}}$ o que é menor ou comparável aos casos Bi-int para os mesmos dopantes que são $E^{F}=1.14 \mathrm{eV}$ e $0.63 \mathrm{eV}$. Os casos substitucionais de ambos são similares aos outros com apenas algumas mudanças na densidade de carga vista nas imagens de STM 32b, 32c, 32d e 32e. A geometria $\mathrm{Co}_{\mathrm{Bi}}\left(\mathrm{Ni}_{\mathrm{Bi}}\right)$ apresenta simetria

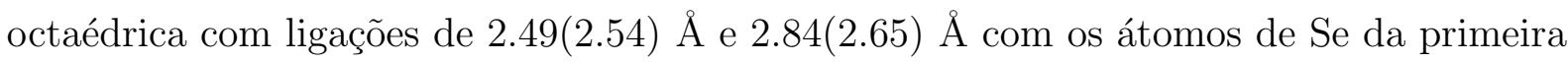
e terceira camadas. Note que os casos substitucionais são comparados aos intersticiais apenas na condição pobre em $\mathrm{Bi}\left(\mu_{\mathrm{Bi}}=\mu_{\mathrm{Bi}}^{\mathrm{min}}\right)$. Na condição estequiométrica definida como $\bar{\mu}_{\mathrm{Bi}}=\left(\mu_{\mathrm{Bi}}^{\max }+\mu_{\mathrm{Bi}}^{\min }\right) / 2$, as energias de $\mathrm{Co}_{\mathrm{Bi}}$ e $\mathrm{Ni}_{\mathrm{Bi}}$ aumentam para $1.35 \mathrm{eV}$ e $0.99 \mathrm{eV}$ tornando as configurações Bi-int as mais prováveis.

A configuração Bi-int para o caso do Co $(\mathrm{Ni})$ que chamamos também de $(\mathrm{Co}-\mathrm{Bi})_{\text {split }}$ $\left((\mathrm{Ni}-\mathrm{Bi})_{\text {split }}\right)$ é muito similar à do Fe, tem coordenação 7, ou seja 3 ligações Co-Se (Ni-Se) de 2.36 (2.39) Å, outras 3 ligações Co-Bi (Ni-Bi) de 3.20 (3.03) A e apenas uma ligação com o Bi da segunda mono camada de 2.57 (2.59) A. As imagens de STM vistas nas figuras 32f, 32g, 32h e 32i são similares para ambos os casos, onde apenas no caso do Co, nota-se uma assimetria devido ao momento magnético alinhado ao plano. 


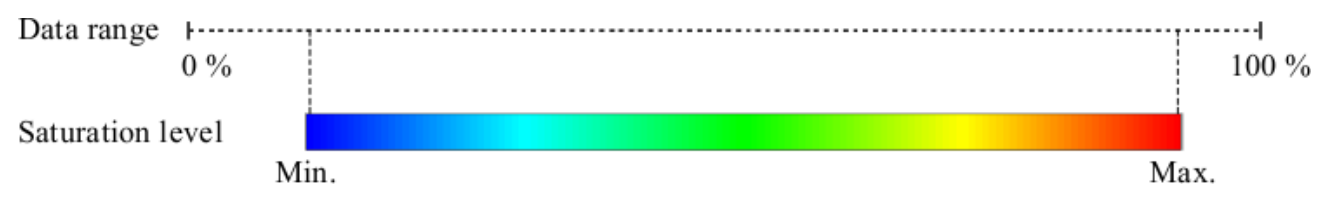

(a) Gradiente de cor no esquema $R-G-B$.

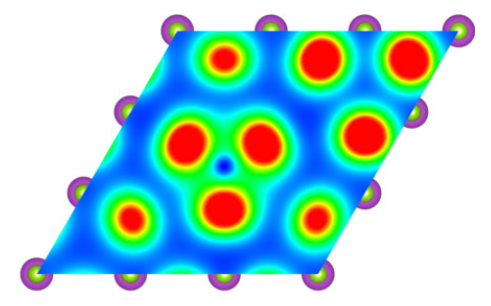

(b) STM est ocu Co-Bi-sub.

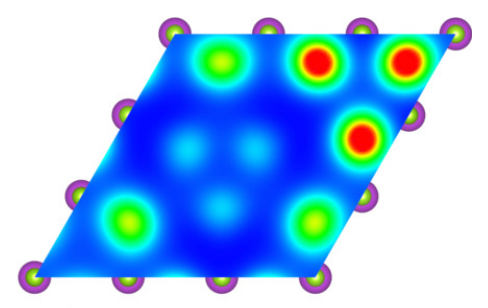

(d) STM est ocu Ni-Bi-sub.

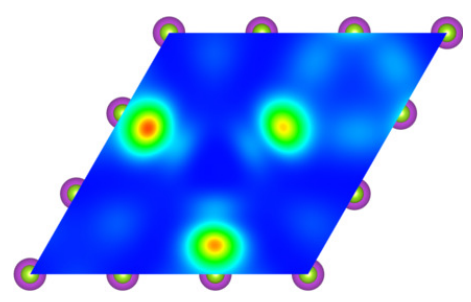

(f) STM est ocu Co-Bi-int.

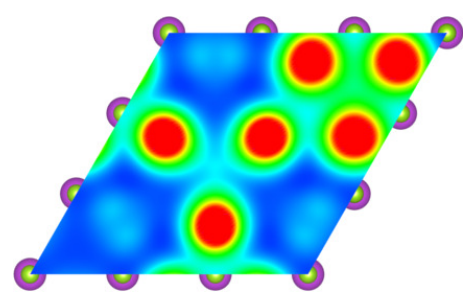

(h) STM est ocu Ni-Bi-int.

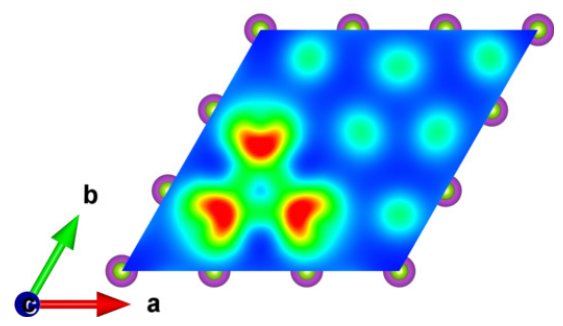

(j) STM est ocu Ni-Se-int.

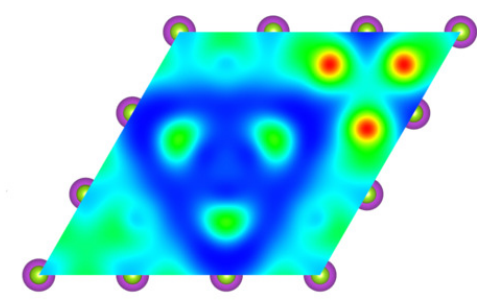

(c) STM est vaz Co-Bi-sub.

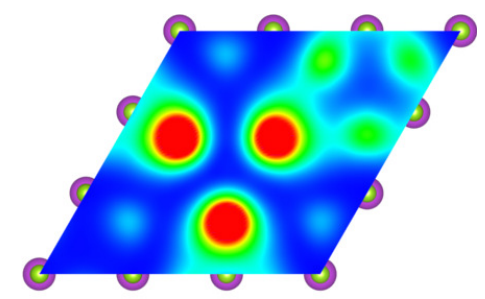

(e) STM est vaz Ni-Bi-sub.

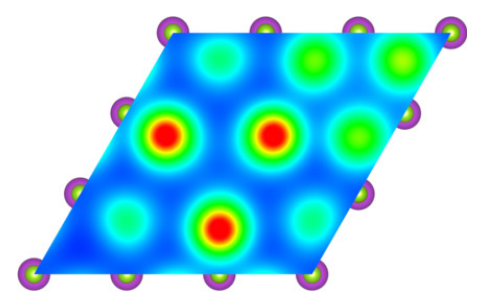

(g) STM est vaz Co-Bi-int.

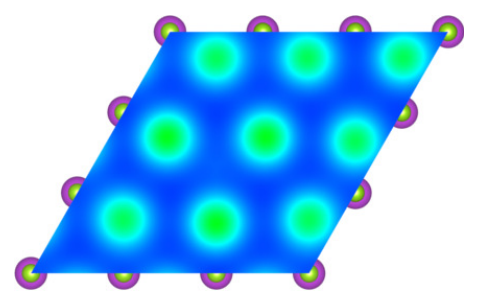

(i) STM est vaz Ni-Bi-int.

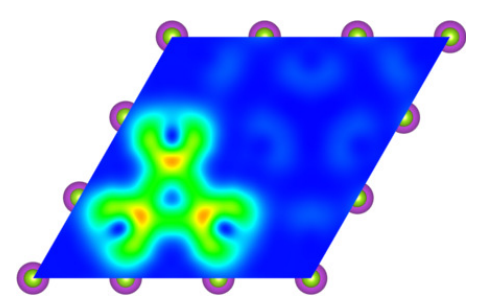

(k) STM est vaz Ni-Se-int.

Figura 32: Mesmo que 31, para outras geometrias. 
Para as impurezas de $\mathrm{Ni}$ as configurações Bi-int e Se-ads são próximas em energia porém apresentam imagens de STM distintas vistas nas figuras 32h, 32i, 32j e 32k. Logo podemos dizer que o $\mathrm{Ni}$ na superfícies de $\mathrm{Bi}_{2} \mathrm{Se}_{3}(111)$ ocupa sítios adsorvidos acima do Se e depois segrega-se em geometrias intersticiais de Bi. Similarmente para o caso de Co temos que a geometria Bi-ads compete com a intersticial, aonde o mesmo cenário pode ocorrer.

Recentemente a formação da geometria Co logo acima do átomo de Se da primeira camada foi proposto [127]. Entretanto como a energia de formação obtida desta geometria, cuja átomo permanecia $2.26 \AA$ acima do primeiro Se, foi de $E^{F}=3.53 \mathrm{eV}$ muito maior que as outras configurações, descartamos esta possibilidade.

\subsubsection{Caracterização topológica de $\mathrm{TM} / \mathrm{Bi}_{2} \mathrm{Se}_{3}(111)$}

De maneira a verificar as mudanças nas propriedades eletrônicas da superfície do $\mathrm{Bi}_{2} \mathrm{Se}_{3}(111)$ na presença dos metais de transição, examinaremos a estrutura de banda eletrônica e as componentes cartesianas da SPDOS, chamadas de $P_{\alpha}(E)$, projetada tanto nas duas primeiras mono-camadas (2ML), quanto na própria impureza.

A preferência de orientação do vetor de polarização de spin foi obtida comparando as energias totais das orientações no plano $\left(\left\langle S_{x}\right\rangle,\left\langle S_{y}\right\rangle\right)$ e fora do plano $\left(\left\langle S_{z}\right\rangle\right)$. É importante notar que devido à metodologia de supercélula, todos os nossos sistemas tem apenas um átomo de impureza cuja orientação magnética é repetida no espaço devido as condições periódicas de contorno, logo os casos que possuem uma magnetização são ferromagnéticos. No caso substitucional os orbitais $\mathrm{Fe}_{\mathrm{Bi}^{-}}-3 \mathrm{~d}$ proporcionam 3 bandas flats desocupadas em $\left(E_{F}+0.3\right) \mathrm{eV}$ indicados por quadrados azuis na figura 33a. Nota-se que dos estados acima o de mais baixa energia hibridiza com os estados das duas primeiras mono-camadas (círculos vermelhos $)$ em $\left(E_{F}+0.25\right) \mathrm{eV}$. Todavia abaixo de $\left(E_{F}+0.20\right) \mathrm{eV}$ a contribuição de Fe-3d é menor que $10 \%$, enquanto que as duas primeiras mono-camadas dominam a formação do cone de Dirac. Achamos também a presença de um pequeno gap de $15 \mathrm{meV}$ no próprio ponto $\Gamma$. Os cálculos de SPDOS revelaram uma polarização de spin fora do plano ao longo das direções $\bar{\Gamma}-\bar{K}$ e $\bar{K}-\bar{M}$, principalmente mediada pela impureza ou seja 


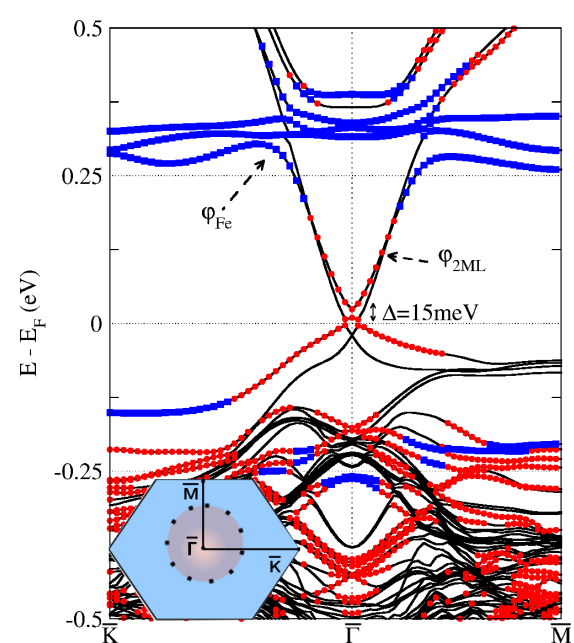

(a) Banda eletrônica de $F e_{B i}$

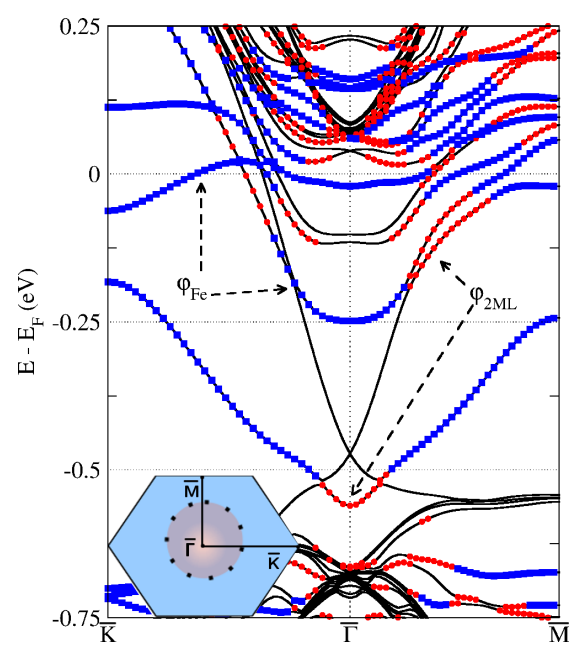

(c) Banda eletrônica de Fe-Bi-int
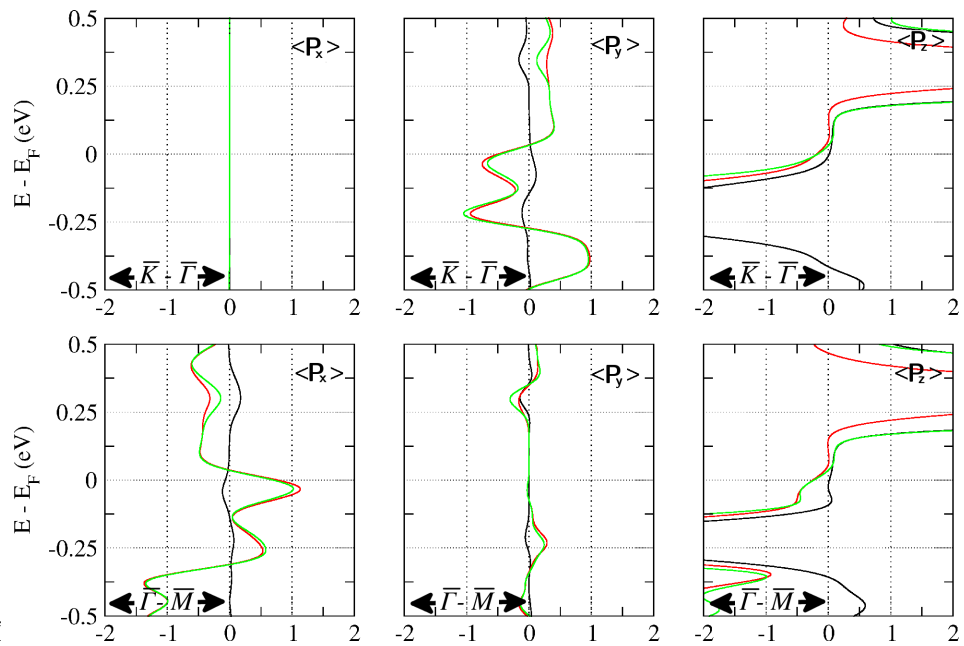

(b) SPDOS nas direções $\bar{\Gamma}-\bar{K}$ e $\bar{K}-\bar{M}$ da banda 33 .
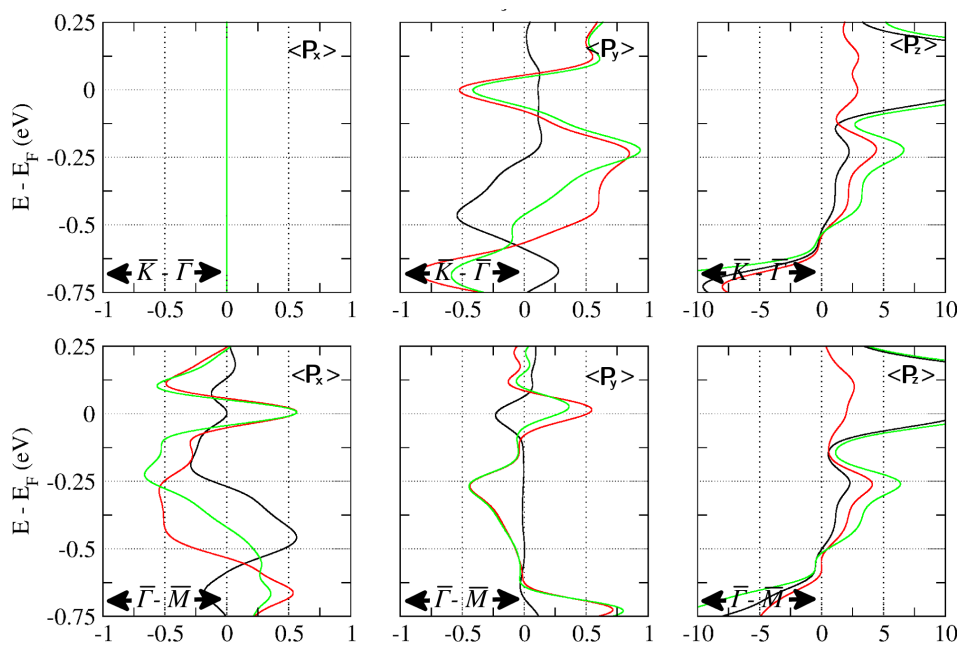

(d) SPDOS nas direções $\bar{\Gamma}-\bar{K}$ e $\bar{K}-\bar{M}$ da banda 33c.

Figura 33: Propriedades eletrônicas dos casos Fe-Bi-sub e Fe-Bi-int. (a)-(b) Bandas de energia com inset da zona de Brillouin e tamanho $\Delta$ do gap. Linhas sólidas pretas nos diagramas de banda indicam a contribuição de todos os átomos para a banda do material, enquanto que círculos vermelhos e quadrados azuis representam respectivamente a contribuição para a banda vinda apenas das primeiras $2 M L$ e do metal de transição, as quais foram relaxadas. Tal contribuição em cada ponto de banda foi selecionada considerando uma porcentagem crítica (maior que 10 \%) da função de partícula única de Kohn-Sham e em (c) e (d) temos a $P_{\alpha}(E)$ projetada, apenas na impureza em linha preta, nas primeiras $2 M L$ em vermelho, e na soma de ambas em verde.

$\left\langle\phi_{\mathrm{Fe}}(\vec{k})|\vec{S}| \phi_{\mathrm{Fe}}(\vec{k})\right\rangle \sim\left\langle S_{z}\right\rangle$ (vista em linhas pretas na figura 33b), assim removendo a TRS. Além disso há uma contribuição $\left\langle S_{z}\right\rangle$ das primeiras 2ML em ambas as direções indicado em vermelho em 33b. Por outro lado as componentes $\left\langle S_{x}\right\rangle,\left\langle S_{y}\right\rangle$ se assemelham ao caso pristino, aonde a contribuição do Fe é praticamente nula.

Para o caso intersticial notamos a formação de um estado metálico onde parte dos estados ocupados são compostos por orbitais Fe-3d hibridizados com orbitais Se-4p da 
superfície. A textura helicoidal, vista no caso pristino é perdida devido à polarização de spin fora do plano em ambas direções como visto na figura 33d. Aqui temos a perda da TRS, além de um deslocamento vertical negativo em energia das banda de condução de superfície. Estes achados estão de acordo com recentes medidas experimentais [102,103], já em outros artigos [101], o cone de Dirac foi mantido para concentrações de Fe até 0.3 ML. Para baixas concentrações de cobertura 0.01ML o cone de Dirac foi mantido, com átomos de Fe situados no sítio Se-ads [114]. Neste último caso a preservação do gap foi atribuída a uma polarização de spin no plano. De fato observamos uma polarização de spin no plano, para átomos adsorvidos, porém não para a configuração Se-ads e sim para Bi-ads que é 120 meV mais estável. Para o caso $F_{\text {Bi }}$ as impurezas são doadoras de elétrons, com estado de ionização de $3^{+}$e uma magnetização $(\mu)$ de $5.0 \mu_{\mathrm{B}}$ (magneton de Bohr), enquanto que para o caso intersticial Bi-int, a segunda configuração mais estável, temos um estado de ionização entre $1^{+}$e $2^{+}$e um $\mu$ de $2.5 \mu_{\mathrm{B}}$. O momento magnético obtido para esta configuração sub em $\mathrm{Bi}_{2} \mathrm{Se}_{3}$ está de acordo com recentes cálculos ab initio [125]. Na referência [102] autores obtiveram um estado de ionização entre $2^{+}$e $3^{+}$ e uma magnetização em torno de $4.0 \mu_{\mathrm{B}}$. Baseado em cálculos similares West et al [126] obtiveram $4.0 \mu_{\mathrm{B}}$ para o caso substitucional $\mathrm{Fe}_{\mathrm{Bi}}$ em $\mathrm{Bi}_{2} \mathrm{Te}_{3}(111)$ enquanto que para a configuração Bi-int eles obtiveram $4.5 \mu_{\mathrm{B}}$. No caso Se-ads temos uma magnetização no plano de $4.0 \mu_{\mathrm{B}}$ verificada em [101] enquanto que na referência [109], autores obtiveram uma magnetização fora do plano de aproximadamente $\mu=2.9 \mu_{B}$ para o caso Bi-ads.

Das referências acima notamos que as interacões químicas entre a impureza e a superfície de $\mathrm{Bi}_{2} \mathrm{Se}_{3}(111)$ têm um papel fundamental nas propriedades eletrônicas e magnéticas de $\mathrm{TM} / \mathrm{Bi}_{2} \mathrm{Se}_{3}(111)$. Para entender melhor o efeito do ambiente cristalino na magnetização destes materiais iremos analisar na figura 34, fazendo o uso de teoria de grupos, a quebra de degenerscência energética dos orbitais $d$ desses materiais sob certas condições. Para o caso substitucional 34a, o TM exibe uma simetria octaédrica proporcionando a partir dos 5 orbitais d, 3 orbitais degenerados de menor energia chamados de " $t$ ", e outros 2 orbitais, denominados " $e$ " de maior energia. Esta quebra de degenerescência 


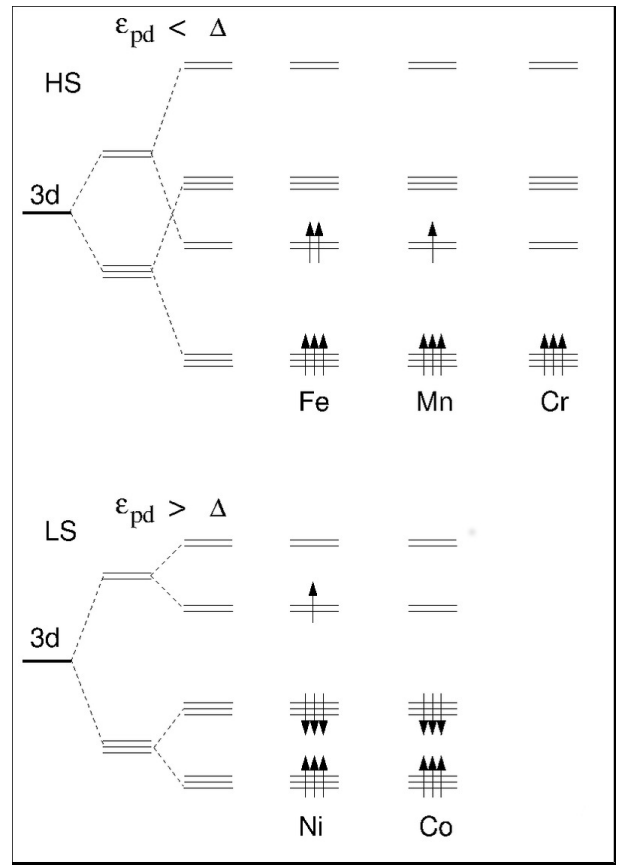

(a) Caso Bi-sub.

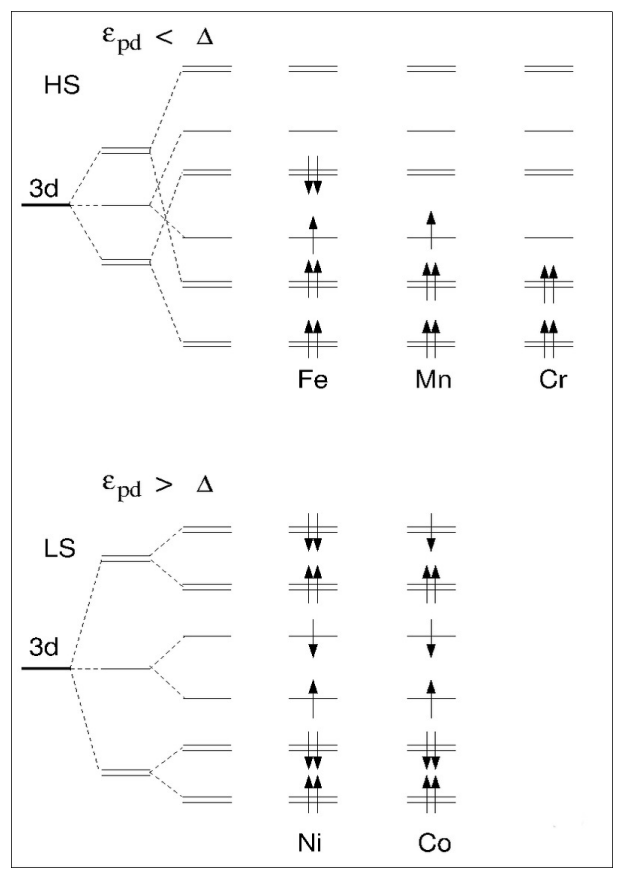

(b) Caso Bi-int.

Figura 34: Quebra de degenerescência dos orbitais atômicos 3d de todos os TM nos ambientes (a) Bi-sub e (b) Bi-int após se ligar respectivamente o campo cristalino e o campo de troca. Em cada caso há duas configurações possíveis, que são High Spin(HS) e Low Spin(LS).

energética $\left(e_{\mathrm{pd}}\right)$ é devida ao campo cristalino induzido pela interação entre os orbitais $3 \mathrm{~d}$ da impureza e os orbitais $4 p$ dos átomos de Se vizinhos. Ao ligar a polarização de spin outras quebras energéticas irão acontecer, devido ao campo de troca ou exchange $(\Delta)$. Neste caso temos duas situações possíveis:

- $e_{\mathrm{pd}}<\Delta$ que corresponde a uma configuração de High Spin (HS).

- $e_{\mathrm{pd}}>\Delta$ que corresponde a uma configuração de Low Spin (LS).

Neste cenário notamos que a magnetização de 5.0, 4.0 e $3.0 \mu_{\mathrm{B}}$ de $\mathrm{Fe}_{\mathrm{Bi}}, \mathrm{Mn}_{\mathrm{Bi}}$ e $\mathrm{Cr}_{\mathrm{Bi}}$ apresentam uma configuração de High Spin. Entretanto para $\mathrm{Co}_{\mathrm{Bi}}$ e $\mathrm{Ni}_{\mathrm{Bi}} \operatorname{com} \mu=0.0 \mu_{\mathrm{B}}$ e $\mu=1.0 \mu_{\mathrm{B}}$ respectivamente concordam com modelos de Low Spin. Na tabela 7, temos os momentos magnéticos e suas respectivas orientações de mais baixa energia sendo $(\leftarrow)$ no plano, e $(\uparrow)$ fora do plano. Similarmente ao caso $\mathrm{Fe}_{\mathrm{Bi}}, \mathrm{Mn}_{\mathrm{Bi}}$ e $\mathrm{Cr}_{\mathrm{Bi}}$ também induzem um pequeno gap no cone de Dirac, de 4 meV (figura 35a) e 8 meV (figura 36a) (lembrando que para o funcional escolhido na metodologia DFT, podemos subestimar o valor real do gap), respectivamente, e seus momentos magnéticos são iguais a 4.0 e $3.0 \mu_{\mathrm{B}}$. Para ambos 


\begin{tabular}{cccccc}
\hline \hline & & & & & \\
Configuração & Cr & Mn & Fe & Co & Ni \\
\hline Bi-sub & $3.0 \uparrow$ & $4.0 \leftarrow$ & $5.0 \uparrow$ & $0.0 \mathrm{x}$ & $1.0 \leftarrow$ \\
Se-sub & $4.0 \leftarrow$ & $3.0 \leftarrow$ & $2.0 \leftarrow$ & $1.0 \leftarrow$ & $0.0 \mathrm{x}$ \\
Bi-int & $4.0 \leftarrow$ & $5.0 \uparrow$ & $2.5 \uparrow$ & $1.5 \leftarrow$ & $0.0 \mathrm{x}$ \\
Se-int & $0.5 \leftarrow$ & $4.0 \uparrow$ & $2.0 \leftarrow$ & $1.5 \leftarrow$ & $0.0 \mathrm{x}$ \\
Bi-ads & $5.0 \uparrow$ & $4.0 \leftarrow$ & $2.5 \leftarrow$ & $1.5 \uparrow$ & $0.1 \leftarrow$ \\
Se-ads & $0.4 \leftarrow$ & $4.0 \uparrow$ & $2.0 \uparrow$ & $1.3 \uparrow$ & $0.0 \mathrm{x}$ \\
\hline
\end{tabular}

Tabela 7: Módulo dos momentos magnéticos $\left(e m \mu_{B}\right)$ e a orientação da polarização líquida, seja ela fora do plano $(\uparrow)$, ou no plano $(\leftarrow)$.

os sistemas os orbitais 3d hibridizam com a superfície que compõe o cone, por outro lado na borda da zona estes orbitais tornam-se flats.

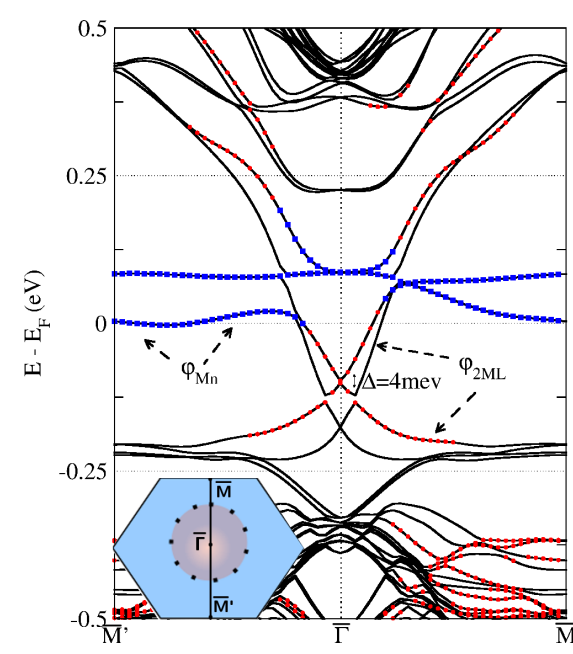

(a) Banda eletrônica de $M n_{B i}$.

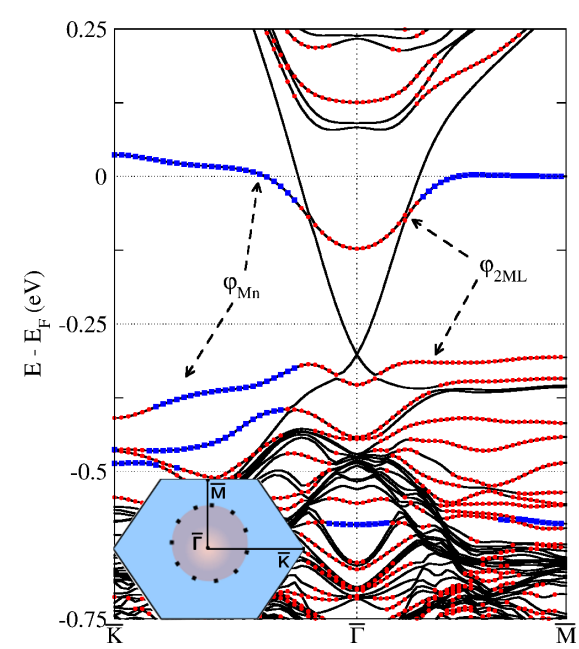

(c) Banda eletrônica de Mn-Bi-int.
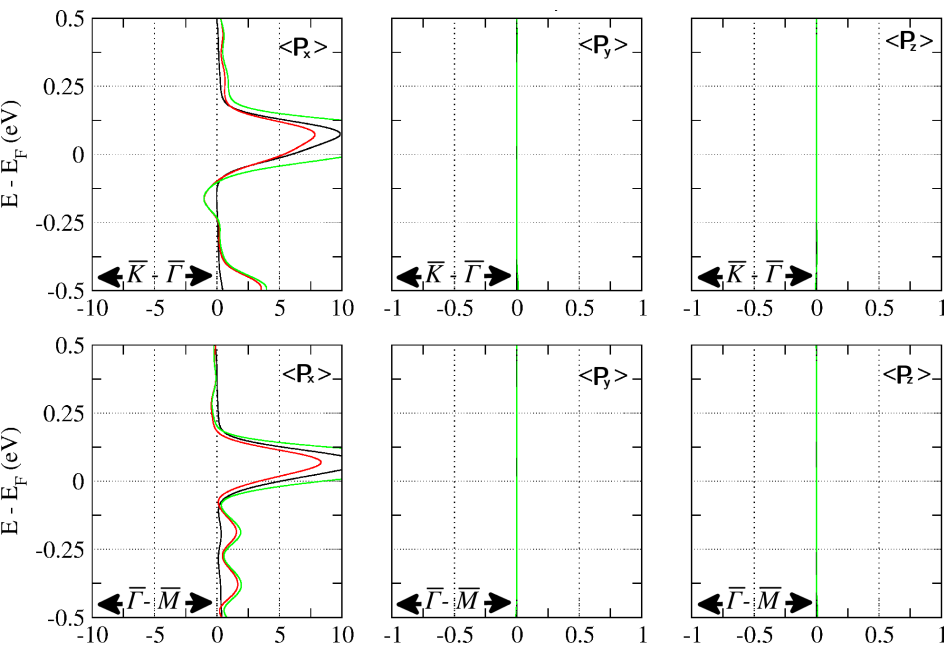

(b) SPDOS nas direções $\bar{\Gamma}-\bar{K}$ e $\bar{K}-\bar{M}$ da banda $35 a$.
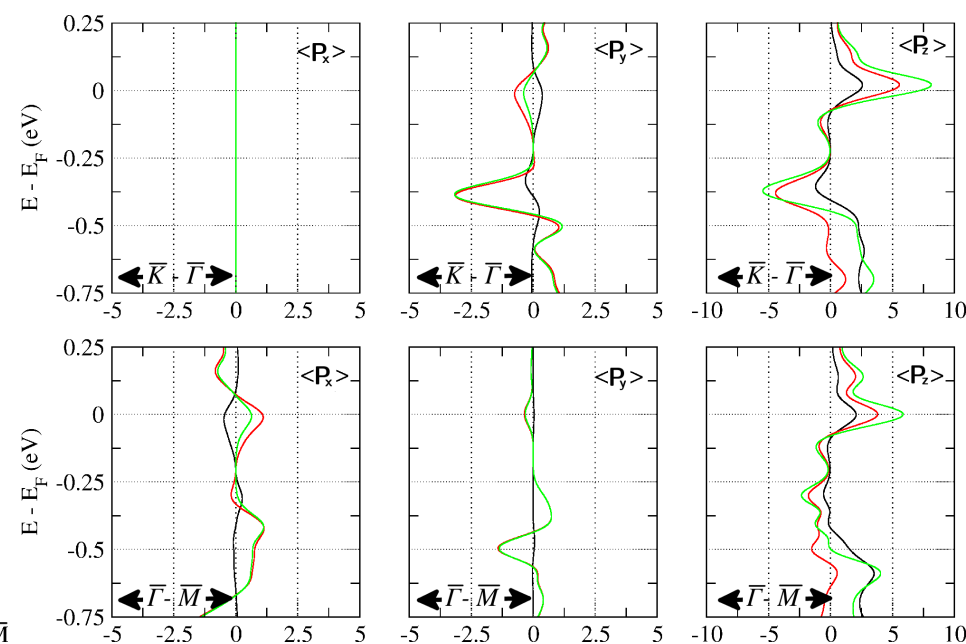

(d) SPDOS nas direções $\bar{\Gamma}-\bar{K}$ e $\bar{K}-\bar{M}$ da banda $35 c$.

Figura 35: Mesmo esquema que para a figura 33, para os casos Mn-Bi-sub e Mn-Bi-int. 
Nossos cálculos de SPDOS revelam a hibridização de impureza com a superfície. Para $\mathrm{Mn}_{\mathrm{Bi}}$ há uma preferência energética para orientações de magnetização no plano, onde os estados da impureza e das primeiras ML contribuem fortemente para a magnetização $\left\langle\phi_{2 \mathrm{ML}}|\vec{S}| \phi_{2 \mathrm{ML}}\right\rangle=\left\langle S_{x}\right\rangle$ enquanto que outras contribuições $\left\langle S_{y}\right\rangle$ e $\left\langle S_{z}\right\rangle$ desaparecem. Já para o caso de $\mathrm{Cr}_{\mathrm{Bi}}$ temos uma preferência magnética para a orientação fora do plano. $\mathrm{O}$ momento magnético calculado foi de $3.0 \mu_{\mathrm{B}}$ que se compara muito bem com achados experimentais [106] e cálculos teóricos ab initio [125]. A presença de um pequeno gap no cone

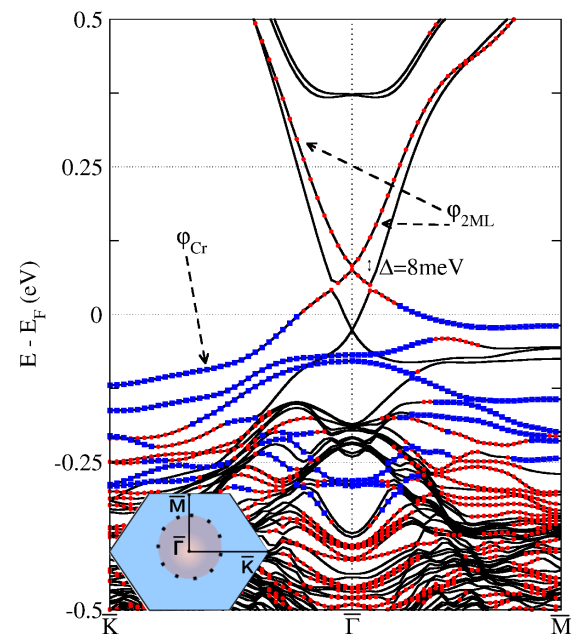

(a) Banda eletrônica de $C r_{B i}$.
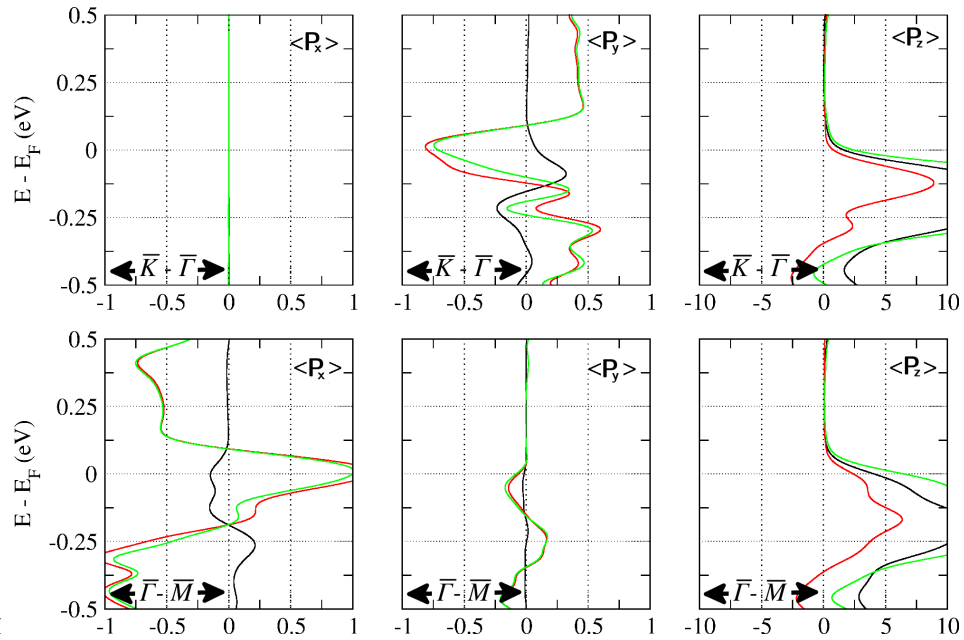

(b) SPDOS nas direções $\bar{\Gamma}-\bar{K}$ e $\bar{K}-\bar{M}$ da banda $36 a$.

Figura 36: Mesmo esquema que para a figura 33, para o caso Cr-Bi-sub.

de Dirac, por volta de $7 \mathrm{meV}$, foi experimentalmente observado para $\mathrm{Mn} / \mathrm{Bi}_{2} \mathrm{Se}_{3}(111)$ ferromagnético [103]. Outros dados experimentais confirmam a formação de um gap de energia no cone de Dirac, quebrando a TRS, indicando a presença da configuração $\mathrm{Mn}_{\mathrm{Bi}}[100]$. Neste último trabalho os autores verificaram uma fase ferromagnética, com momento magnético de $3.45 \mu_{\mathrm{B}}$, porém devido à inomogeneidade da distribuição de $\mathrm{Mn}$, eles possuem orientações de magnetização tanto no plano como fora dele. Recentemente uma textura de spin espiral quebrando a TRS foi proposta para $\mathrm{Mn} / \mathrm{Bi}_{2} \mathrm{Se}_{3}(111)$, com combinações de magnetização dentro e fora do plano [104]. Nossos resultados mostram que existe uma dependência de geometria local com a magnitude e orientação do momento magnético. Por exemplo o caso $\mathrm{Mn}_{\mathrm{Bi}}$ o $\langle\vec{S}\rangle$ está no plano, já o caso $(\mathrm{Mn}-\mathrm{Bi})_{\mathrm{split}} \mathrm{o}$ $\langle\vec{S}\rangle$ está fora do plano. No último, o cone é perdido como visto na figura 35c, parte dos 
orbitais 3d são ocupados, e a textura helicoidal de spin também é perdida (figura 35d). Para outras geometrias, Se-int Mn apresenta um momento magnético no plano de 4.0 $\mu_{\mathrm{B}}$, para os casos Bi-ads e Se-ads cujas energias de formação são próximas $\left(E^{F}=0.74\right.$ e $0.87 \mathrm{eV}$ respectivamente) temos que ambos têm magnetização e $4.0 \mu_{\mathrm{B}}$, porém no plano para o primeiro e fora dele para o segundo, o que confirma a dependência especial do momento magnético em $\mathrm{Mn} / \mathrm{Bi}_{2} \mathrm{Se}_{3}(111)$ [100]. Para os sistemas cuja energia de formação é menor nos casos int-Bi, temos uma geometria com simetria local próxima a $C_{3 v}$, onde o splitting dos orbitais está descrito na figura 34b. Nota-se que ambas impurezas de Co e Ni apresentam uma configuração de Low Spin e momentos magnéticos de 1.5 e $0.0 \mu_{\mathrm{B}}$ respectivamente. A presença de Co intersticial no sítio de Bi torna a superfície metálica, visto na figura 37a. A figura 37b mostra a preferência energética para uma magnetização no plano dominado pelo Co em ambas as direções. Similarmente ao caso $M n_{B i}$ as outras componentes de $\langle\vec{S}\rangle$ são praticamente nulas. A magnetização local depende do ambiente cristalino onde, por exemplo, as geometrias adsorvidas que são as segundas mais prováveis temos uma preferência energética por uma magnetização fora do plano, já para a outra geometria intersticial em sítio de Se essa preferência torna-se no plano. Em grande contraste com as outras geometrias o cone de Dirac foi mantido para impurezas de Ni int-Bi o cone de Dirac foi para $E^{F}=-0.25 \mathrm{eV}$, indicando um caráter de doador de elétrons. Nota-se uma clara contribuição do Ni na formação do cone visto na figura 37c. Para este sistema vemos na figura 37d que a textura helicoidal de spin foi mantida devido à preservação da TRS. 

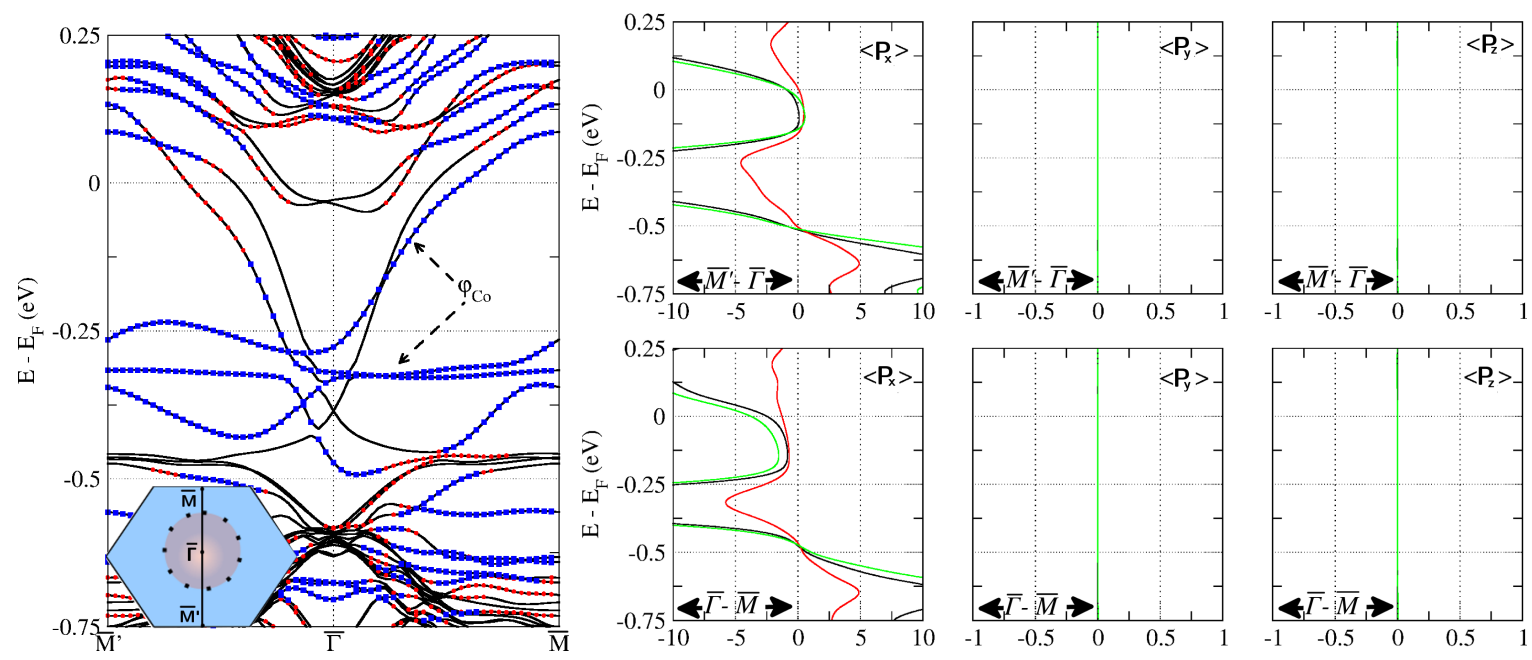

(a) Banda eletrônica de Co-Bi-int.

(b) SPDOS nas direções $\bar{\Gamma}-\bar{K}$ e $\bar{K}-\bar{M}$ da banda 37a.
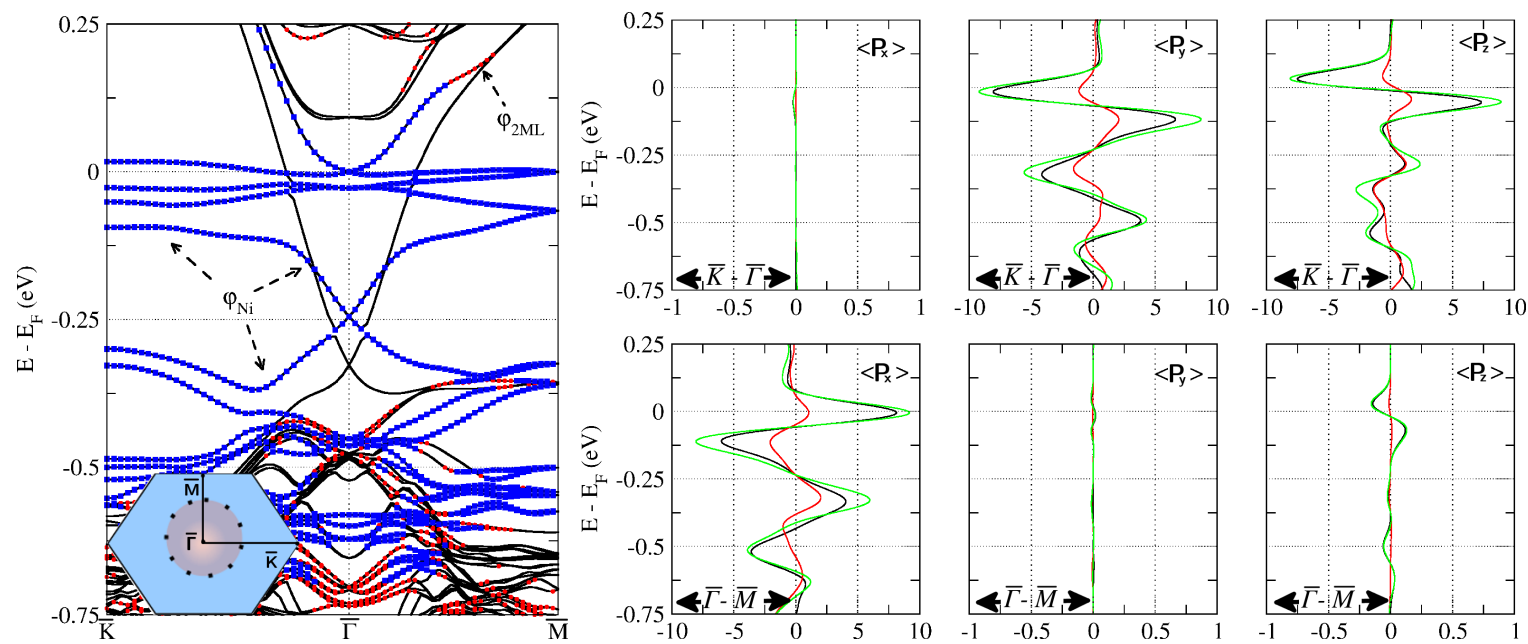

(c) Banda eletrônica de Ni-Bi-int.

(d) SPDOS nas direções $\bar{\Gamma}-\bar{K}$ e $\bar{K}-\bar{M}$ da banda $3 \%$.

Figura 37: Mesmo esquema que para a figura 33, para os casos Co-Bi-int e Ni-Bi-int.

\subsection{Conclusões parciais}

Baseado em cálculos de primeiros princípios, proporcionamos um mapeamento de estabilidade energética e propriedades eletrônicas da superfície de $\mathrm{Bi}_{2} \mathrm{Se}_{3}(111)$ dopada com metais de transição. Inicialmente com o auxílio de cálculos de energia de formação as configurações mais prováveis que foram identificadas se resumem a:

- Bi-sub para, Cr, Mn e Fe na condição rica em Bi.

- Na condição pobre em Bi as impurezas Co e Ni também exibem uma preferência energética por sítios sub-Bi. 
- Na condição estequiométrica a configuração Bi-int tornou-se mais estável tanto para Co quanto para Ni.

Para Fe e Mn, cuja discussão na literatura é maior, existem vários sítios energeticamente próximos em energia aos mais estáveis, sugerindo uma variada gama de possibilidades para estas impurezas. Situação parecida ocorre com Co e Ni onde geometrias adsorvidas têm energia de formação similares a dos mais estáveis. Em constraste esta variedade não é esperada para o caso de Cr.

As propriedades eletrônicas dos estados topológicos de superfície mudam drasticamente na presença de tais impurezas. De fato, nossas imagens de STM indicam que cada sistema $\mathrm{TM} / \mathrm{Bi}_{2} \mathrm{Se}_{3}(111)$ exibe um mapeamente eletrônico único em função da espécie atômica TM e sua posição de equilíbrio.

Análises de estrutura de banda revelaram que impurezas substitucionais como $\mathrm{Fe}_{\mathrm{Bi}} \mathrm{e}$ $\mathrm{Cr}_{\mathrm{Bi}}$ apresentam uma polarização de spin fora do plano, proporcionando um pequeno gap no cone de Dirac. Notamos que nos casos substitucionais o formato do cone é mantido quando comparado com o caso pristino.

De qualquer forma com a exceção de $\mathrm{Ni} / \mathrm{Bi}_{2} \mathrm{Se}_{3}(111)$ os estados de superfície não são mais topologicamente protegidos devido à perda da TRS. Além disso existe uma forte dependência de orientação de polarização de spin com o ambiente cristalino, onde geometrias próximas em energia apresentam orientações e magnitudes de momento magnético distintas. Este trabalho foi publicado na revista Physical Review B [128]. Também são autores Leandro Seixas, Tomé Schmidt, Roberto Miwa e Adalberto Fazzio. 

$\Gamma_{\text {Capítulo }}$

\section{Supressão dos canais de espalhamento em ligas de $\left(B i_{1-x} S b_{x}\right)_{2} S e_{3}$}

Hoje em dia, os isolantes topológicos 3D mais estudados são os compostos binários $\mathrm{Bi}_{2} \mathrm{Se}_{3}$ e $\mathrm{Bi}_{2} \mathrm{Te}_{3}$ os quais são isolantes topológicos fortes. Estes materiais apresentam um gap de energia na sua fase bulk de $0.3 \mathrm{eV}$ e $0.13-0.17 \mathrm{eV}$, respectivamente, uma característica importante para aplicações em dispositivos de spintrônica [113,128-132]. No entanto, o principal desafio nestes sistemas é a de evitar condutividade de canais vindas do bulk, o que impedem um transporte eletrônico eficiente via estados de superfície [19], uma vez que o ponto de Dirac nestes materiais se encontra abaixo de bandas de bulk massivas. Para superar este problema, muitas propostas foram feitas para mover o ponto de Dirac acima dos estados de bulk. Um processo para separar tais bandas é a construção de ligas destes materiais [133-138,138-140]. No entanto, pouco tem sido feito para o isolante topológico com maior gap $\mathrm{Bi}_{2} \mathrm{Se}_{3}$, que também tem estados massivos próximos ao ponto de Dirac.

Neste trabalho, estudamos a transição de fase topológica e a posição energética do ponto de Dirac na liga $\left(\mathrm{Sb}_{x} \mathrm{Bi}_{1-x}\right)_{2} \mathrm{Se}_{3}$. Consideramos concentrações de liga $(x)$ a partir de $x=0, \mathrm{Sb}_{2} \mathrm{Se}_{3}$ (isolante trivial) até $x=1, \mathrm{Bi}_{2} \mathrm{Se}_{3}$ (isolante topológico). A fim de proporcionar uma descrição apropriada da liga $\left(S b_{x} B i_{1-x}\right)_{2} S e_{3}$, as configurações atômicas foram geradas usando a abordagem SQS (Special Quasirandom Structure) explicado no apêndice B. A transição de fase topológica foi verificada por meio de (i) o fechamento 
do gap na fase bulk em função da concentração de Bi $(x)$, com base nos argumentos de continuidade adiabático e de inversão de bandas, e (ii) o cálculo dos invariantes topológicos $\left(\nu_{0}, \nu_{1} \nu_{2} \nu_{3}\right)$. Ao aumentar a concentração de Bi, mostra-se que existe uma transição de fase topológica, quando $x$ está em torno de 0.4. De fato $\left(\mathrm{Sb}_{x} \mathrm{Bi}_{1-x}\right)_{2} \mathrm{Se}_{3}$ apresenta estados de superfície topologicamente protegidos quando $x>0.4$. O mais interessante acontece na faixa de concentração $0.4<x<0.7$ onde verificamos que o cone de Dirac está dentro do gap da fase bulk. Assim, medidas de transporte eletrônicos, nesta faixa de composição da liga, iriam capturar apenas efeitos da superfície.

\subsection{Metodologia}
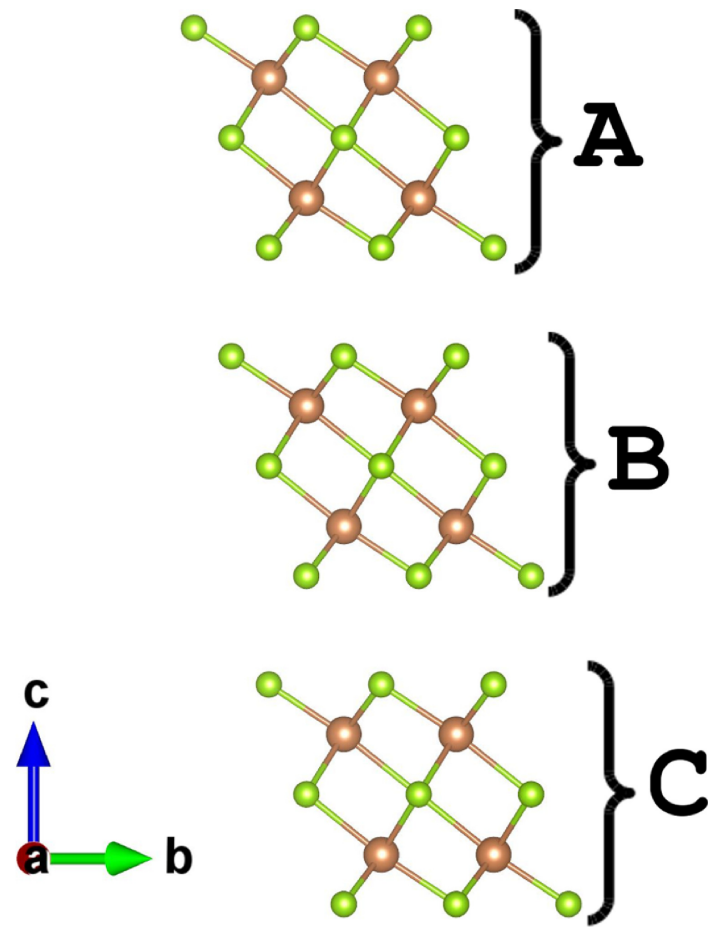

(a) Célula unitária de $\mathrm{Sb}_{2} \mathrm{Se}_{3}$.
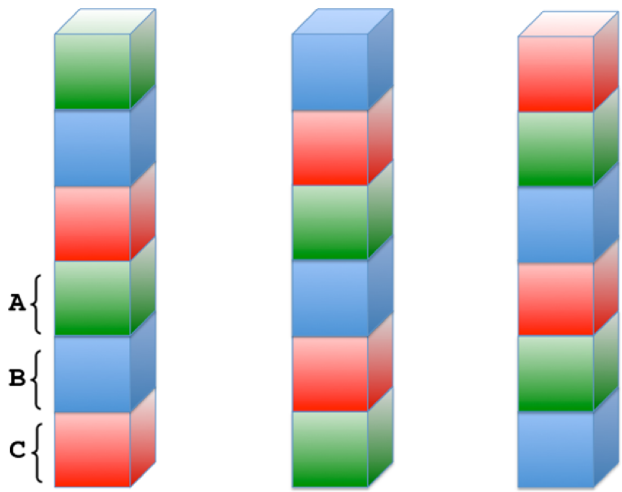

(b) Possiveis superfícies.

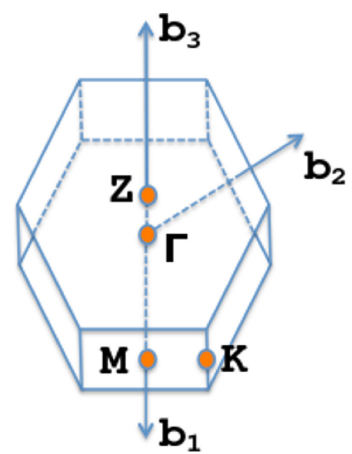

(c) $Z B$ do bulk.

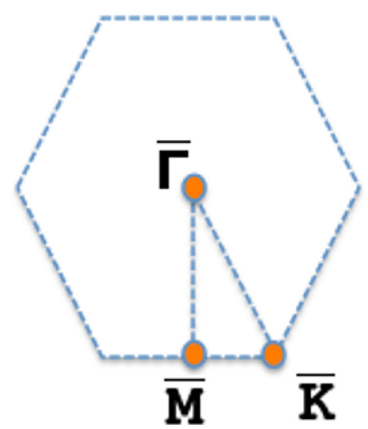

(d) $Z B$ da superficie.

Figura 38: (a) Célula unitária da geometria $\mathrm{Sb}_{2} \mathrm{Se}_{3}$ com seus $3 \mathrm{QLs}$, com parâmetro de rede hexagonal. Aqui podemos ver três diferentes QLs cada uma girada por $120^{\circ}$ graus em relação à próxima. (b) A fim de evitar falhas de empilhamento decidimos criar todas as configurações possiveis de superfícies. Sabendo que nós temos 3 QLs inequivalentes, só é possivel fazer superfícies com terminaçóes do tipo $A-B, A-C$ e $B$-C, como pode ser visto pela escolha de cor diferente para cada QL. (c) Zona de Brillouin da célula de bulk hexagonal com os pontos de alta simetria. (d) Projeção da zona de Brillouin 3D, em sua superfície, com os pontos de alta simetria projetados. 
Cálculos foram feitos no âmbito de DFT [32,34], usando o método do pseudopotencial com PAW [59,60] para descrever as interações elétron íon. A função de troca-correlação foi calculada pela aproximação GGA-PBE [46], como implementado no código VASP [30]. Os orbitais de Kohn-Sham foram expandidos em uma base de ondas planas até um corte em energia de $270 \mathrm{eV}$. A zona de Brillouin foi escolhida por um mesh centrado no ponto $\Gamma$, usando um número de pontos $\vec{k}$ até $4 \times 4 \times 4$ para as contas de bulk, e $4 \times 4 \times 1$ para as superfícies, incluindo o ponto $\Gamma$, até que a energia total estivesse convergida.

As estruturas utilizadas nos cálculos foram construídas através do procedimento SQS [141]. Utilizamos uma super célula $2 \times 2 \times 1$ com 3 QLs, como representado nas figura 38a, na qual substituímos apenas átomos de Bi por Sb, mantendo os átomos Se como espectadores. Consideramos até 16 diferentes concentrações, que vão de 0 \% a $100 \%$ em saltos de 4,2 \%. Para os parâmetros de rede $a$ e $c$ da célula hexagonal, foram utilizados os dados experimentais para $\mathrm{Bi}_{2} \mathrm{Se}_{3}(a=4.138 \AA$ e $c=28.640 \AA)$ [84], e devido à falta de trabalhos experimentais, para o $\mathrm{Sb}_{2} \mathrm{Se}_{3}$ usamos os parâmetros da referência [142] ( $a=$ $4.076 \AA$ e $c=30.90 \AA$ ). Os parâmetros de rede de outras concentrações foram escolhidas com uma função linear cujos limites eram dados pelos sistemas pristinos, seguindo a lei de Vegard, mantendo a simetria hexagonal [137,143].

Nos bulks criados todos os átomos foram permitidos relaxar até as forças atômicas serem menores que $10 \mathrm{meV} / \AA$. Com estas geometrias duplicamos a célula no eixo $z$ (direção (111) da geometria romboédrica), para manter a proporção de átomos, e adicionamos 12 Å de vácuo para poder simular as superfícies, na qual não houve relaxação. Dentro desta abordagem, consideraram três finais de superfície diferentes, como mostrado esquematicamente na figura $38 b$.

Para determinar o número invariante topológico $Z_{2}$, implementamos no código VASP o procedimento de cálculo proposto por Fu e Kane [66]. Este método serve para qualquer código baseado em ondas planas e a metodologia para este cálculo está presente no apêndice D. 


\subsection{Resultados e discussão}

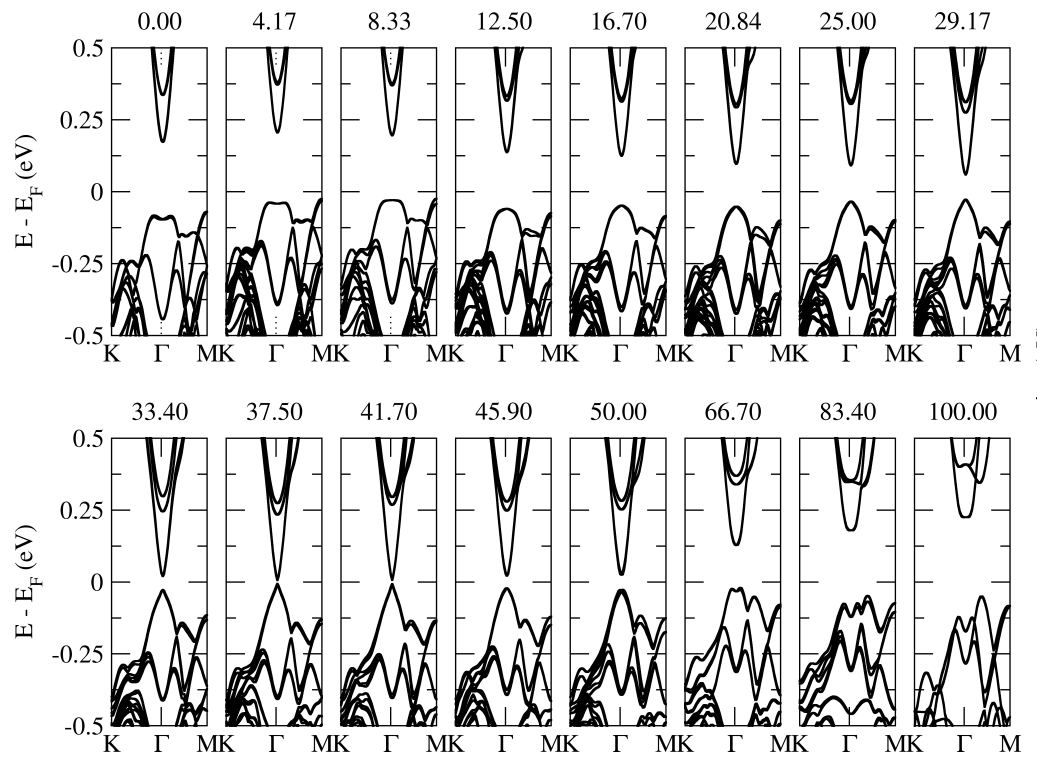

(a) Estrutura de banda do bulk.

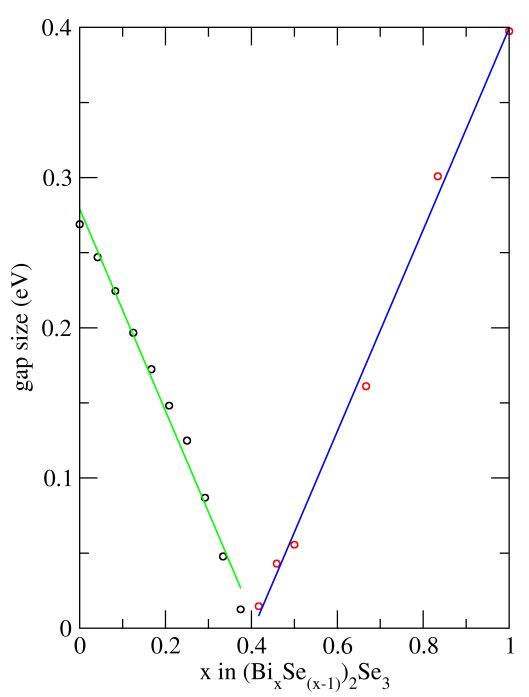

(b) Variação do gap.

Figura 39: (a) Estrutura de banda do bulk para a liga $\left(B i_{x} S b_{1-x}\right)_{2} S e_{3}$, entre $0 \%$ e $100 \%$. (b) variação do gap de $\left(B i_{x} S b_{1-x}\right)_{2} S e_{3}$. Em verde, temos uma linha reta encaixando todos os tamanhos de gap decrescentes, e em azul, temos outra linha ajustando valores com tamanhos de gap crescente.

Na figura 39 apresentamos a evolução da estrutura de banda (figura 39a), e do tamanho do gap (figura 39b) em função da concentração $x$ de Bi, indo desde $x=0$ até $x=1$. Como podemos ver, através do aumento da concentração de Bi o gap do sistema diminui até $40 \%$ e começa a aumentar novamente. Este comportamento do gap em forma de $\mathrm{V}$ é característico de uma transição de fase topológica que podemos entender usando o argumento de continuidade adiabática. Ele afirma que, se um hamiltoniano de um sistema é adiabaticamente transformado em outro sem fechar o gap e sem mudar as simetrias originais [144], o sistema deve compartilhar a mesma classe topológica. Por outro lado, durante esta transformação, se o intervalo de banda fecha, ocorrerá um mudança de fase topológica. Com base neste argumento, sabendo que para $x=1$ temos o $\mathrm{Bi}_{2} \mathrm{Se}_{3}$ que é uma TI, enquanto que para $x=0$ temos $\mathrm{O} \mathrm{Sb}_{2} \mathrm{Se}_{3} \mathrm{um}$ isolante trivial, podemos concluir que uma concentração de Bi de 40 \% o sistema passa por uma transição topológica [145].

O argumento da continuidade adiabática é uma assinatura forte de uma transição de fase topológica. Outro indicativo é uma inversão de banda devido às interações spin- 
órbita [17]. Para $\mathrm{Bi}_{2} \mathrm{Se}_{3}$ existe uma inversão de orbitais $p_{z}$, enquanto que para $\mathrm{Sb}_{2} \mathrm{Se}_{3}$ tal inversão não existe como se mostra na figura 40a e 40d. Também apresentamos na figura 40b e 40c a projeção dos orbitais $p_{z}$ para concentrações de $37.5 \%$ e $45.9 \%$, respectivamente. Nós encontramos que para o primeiro não existe tal inversão orbital, enquanto que para a concentração de Bi de $45.9 \%$, o nivelamento dos orbitais $p_{z}$ está invertido próximo ao ponto $\Gamma$. A fim de fornecer um apoio adicional à transição trivial $\rightarrow$ TI, calculamos os números $\left(\nu_{0}, \nu_{1} \nu_{2} \nu_{3}\right)$ para $\left(\mathrm{Bi}_{x} \mathrm{Sb}_{1-x}\right)_{2} \mathrm{Se}_{3}$ em função da concentração da liga. Encontramos $\left(\nu_{0}, \nu_{1} \nu_{2} \nu_{3}\right)=(0,000)$ para a concentração Bi até $40 \%$, enquanto que para as concentrações maiores do que $40 \%$ obtivemos $\left(\nu_{0}, \nu_{1} \nu_{2} \nu_{3}\right)=(1,000)$. Assim, com base no argumento adiabático, e na avaliação dos números $\left(\nu_{0}, \nu_{1} \nu_{2} \nu_{3}\right)$ mapeamos com grande precisão a transição trivial $\rightarrow \mathrm{TI}$ em $\left(\mathrm{Bi}_{x} \mathrm{Sb}_{1-x}\right)_{2} \mathrm{Se}_{3}$ em função da concentração da liga.

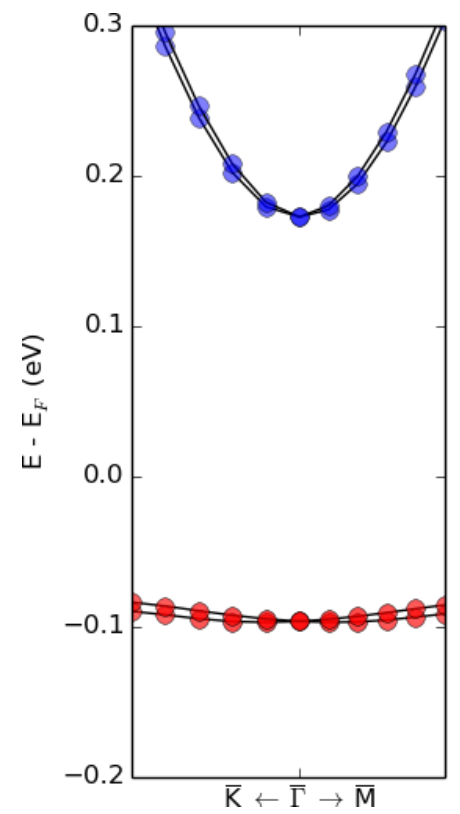

(a) $x=0$.

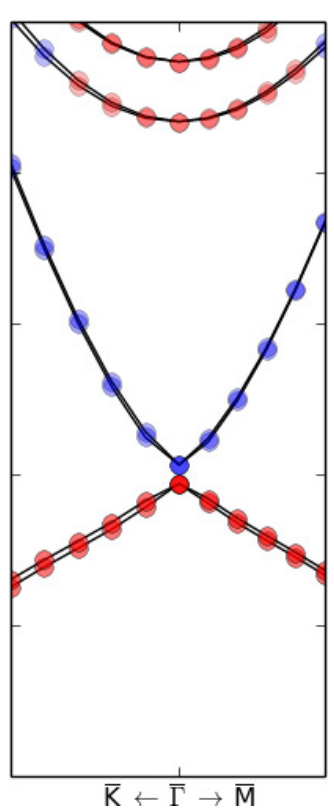

(b) $x=0.375$

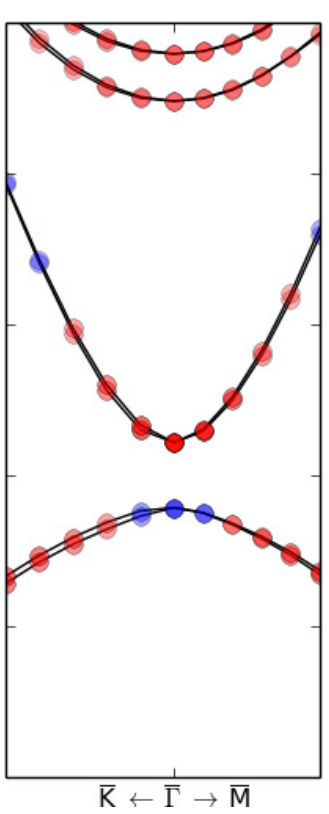

(c) $x=0.459$.

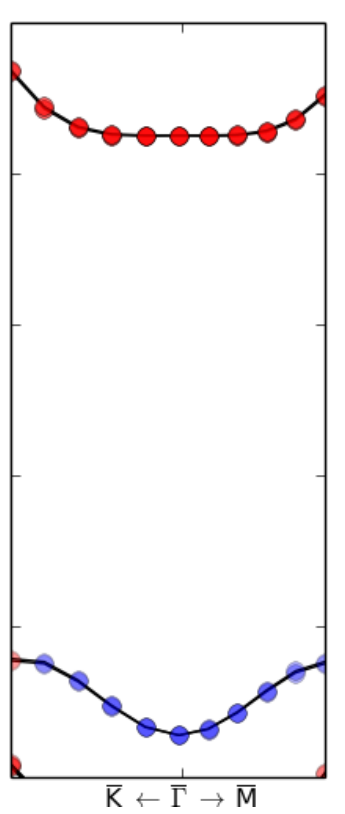

(d) $x=1$.

Figura 40: Estrutura de banda em torno do nível de Fermi de $\left(B i_{x} S b_{1-x}\right)_{2} S e_{3}$ para (a) $x=0$, (b) $x=0.375$, (c) $x=0.459$, (d) e $x=1$. Os orbitais $p_{z}$ de Bi/Sb são indicados por pontos azuis, enquanto que os orbitais $p_{z}$ do Se são indicados por pontos vermelhos. Ambas as cores possuem um esquema de transparência onde a cor sólida representa $100 \%$ de contribuição e transparente $0 \%$.

Uma das principais características em isolantes topológicos, é a presença de estados metálicos protegidos na superfície. Calculou-se a estrutura de banda de superfície de 


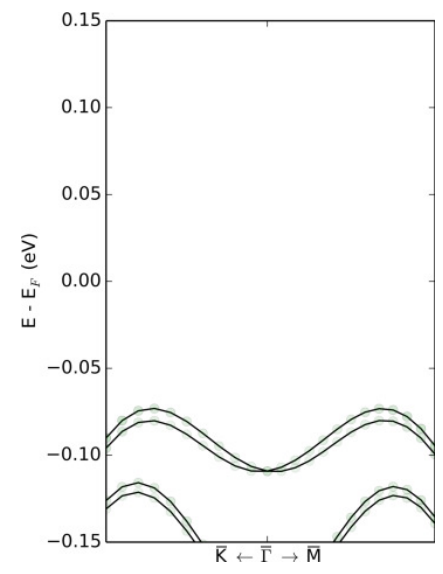

(a) $x=0$.

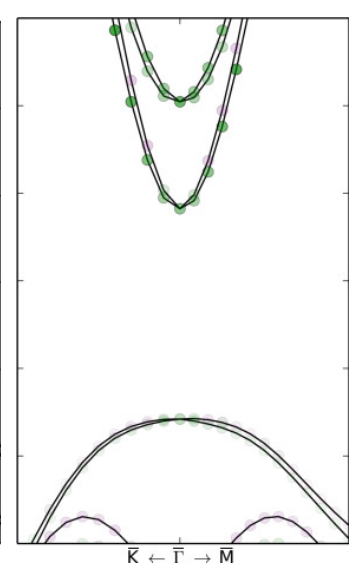

(b) $x=0.33$.

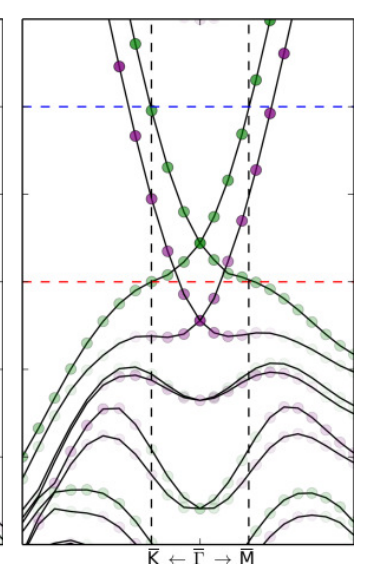

(c) $x=0.67$.

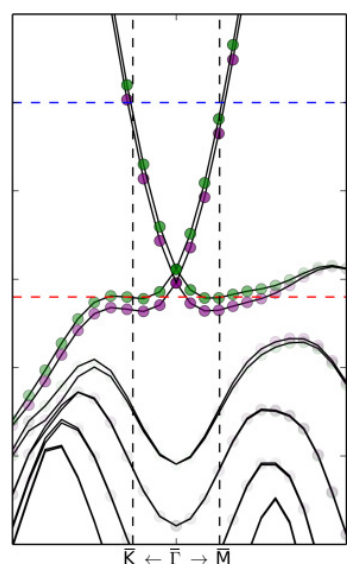

(d) $x=1$.

Figura 41: Estruturas de banda de superfície de (Bi $\left.i_{x} S b_{1-x}\right)_{2} \mathrm{Se}_{3} \mathrm{com}$ (a) $x=0$ ( $\left.\mathrm{Sb}_{2} \mathrm{Se}_{3}\right)$, (b) $x=0.33$, (c) $x=0.67, e$ (d) $x=1$ (Bi $\left.i_{2} S e_{3}\right)$. Em roxo (verde) temos a projeção da banda nas três primeiras (últimas) monocamadas do material. Em (c) - (d) temos as referências em energia da figura 43. Ambas as cores possuem um esquema de transparência onde a cor sólida representa $100 \%$ de contribuição e transparente $0 \%$.

$\left(\mathrm{Bi}_{x} \mathrm{Sb}_{1-x}\right)_{2} \mathrm{Se}_{3}$ para várias concentrações de $x$ entre 0 e 1 . A formação desses estados protegidos pode ser modelada pela concentração da liga. De fato, como mostrado na figura 41a não há estados metálicos de superfície dentro do gap de bulk de $\mathrm{Sb}_{2} \mathrm{Se}_{3}$. Ao aumentar a concentração de Bi para $x=0.375$, visto na figura 41b, uma tendência do gap se fechar ocorre, no entanto, o estado metálico na superfície ainda está ausente. Acima de certa concentração de Bi $(x>0.4)$ temos a origem dos estados topologicamente protegidos como representado na figura 41c $(x=0.667)$. Aqui consideramos nove QLs para calcular a banda de estrutura eletrônica da superfície de forma a remover o pequeno gap devido aos efeitos de interação entre as superfícies. Quanto maior a porcentagem de Sb, maior será a quantidade de QLs para se criar os estados de superfície. Os estados metálicos são preservados até $x=1$, figura 41d. É importante notar que, de acordo com trabalhos anteriores, o Ponto de Dirac (DP) está localizado abaixo da banda de valência de maior energia, figura 41d. No entanto, descobrimos que para concentrações de Bi entre $0.4<x<0.7$, o DP permance acima destes estados de valência vindas do bulk (figura 41c). Este efeito pode ser observado na figura 42, onde em vermelho temos a banda da superfície, e em pretos os estados de bulk projetados na superfície. Nota-se que na figura 42c existe um região de estados metálicos de superfície totalmente inseridas no gap de 


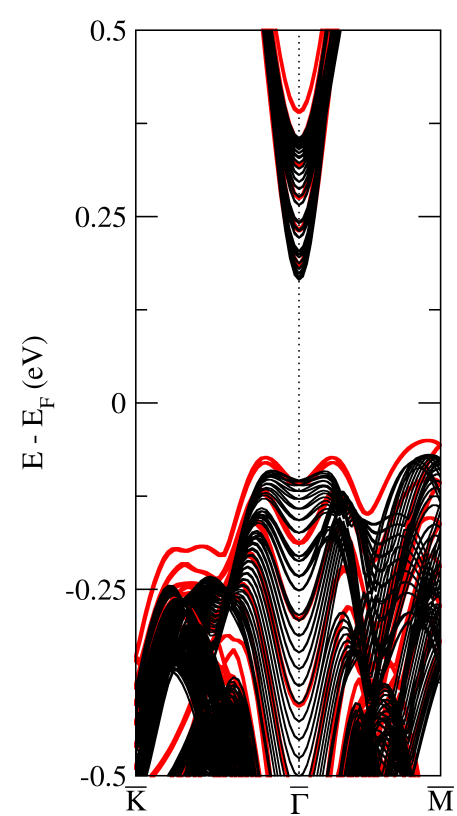

(a) $x=0$.

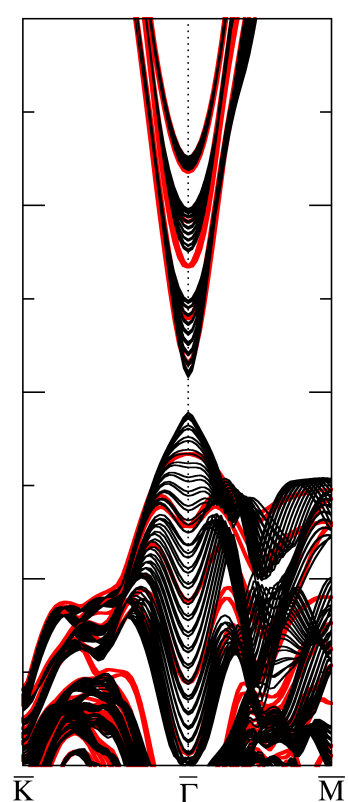

(b) $x=0.33$.

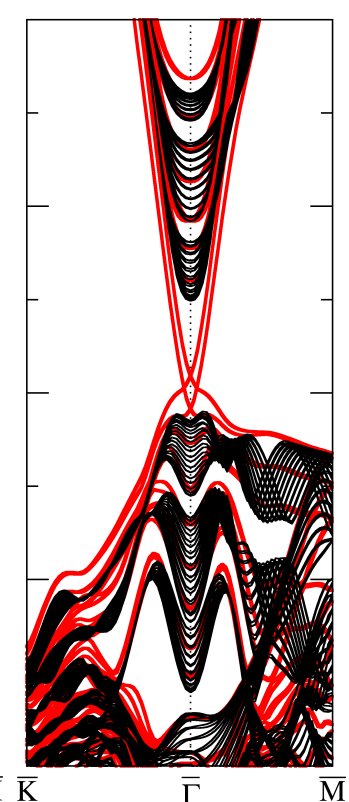

(c) $x=0.67$.

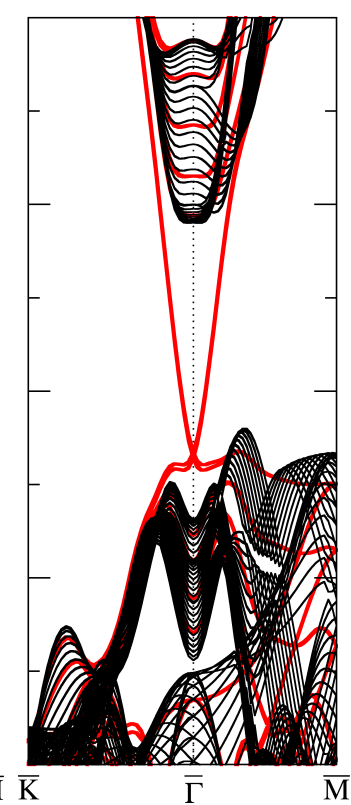

(d) $x=1$.

Figura 42: Bandas das mesmas concentrações que na figura 40, mapeadas na zona de Brillouin inteira. Aqui em vermelho temos a banda de superfície, e em preto a projeção dos estados de bulk. Nota-se a interferência de estados do bulk, em altas concentrações de Bi, podendo proporcionar uma condutividade através o material inteiro.

energia do bulk. O deslocamento vertical positivo em energia do DP em relação ao estados de bulk mediante a presença do átomo de $\mathrm{Sb}$ na liga de $\left(\mathrm{Bi}_{x} \mathrm{Sb}_{1-x}\right)_{2} \mathrm{Se}_{3}$, pode ser atribuído ao SOC mais fraco no Sb em comparação como nos átomos de Bi [137].

Além disso, verifica-se que não apenas a dispersão de energia pode ser controlada pela concentração da liga, mas também encontramos diferentes imagens de textura de spin no cone de Dirac de $\left(\mathrm{Bi}_{x} \mathrm{Sb}_{1-x}\right)_{2} \mathrm{Se}_{3}$ em função da concentração da liga. Na figura 43 apresentamos a textura helicoidal de spin do sistema $\left(\mathrm{Bi}_{x} \mathrm{Sb}_{1-x}\right)_{2} \mathrm{Se}_{3}$ para $x=1$ na figura 43a e $x=0.667$ na figura 43b. Aqui mapeamos a textura de spin em uma fatia de energia da estrutura de banda de superfície perto do nível de Fermi. Notamos que no sistema $\mathrm{Bi}_{2} \mathrm{Se}_{3}$ pristino, as contribuições $S_{x}$ e $S_{y}$ estão trancadas no plano $\hat{x} \cdot \hat{y}$, mas há uma contribuição $S_{z}$ que oscila em volta do cone Dirac. Esta última contribuição tem valores positivos e negativos além de passar por alguns nós. A conseqüência é um vetor de spin $\vec{S}$ inclinado a partir do plano $\hat{x} \cdot \hat{y}$, em alguns pontos da zona de Brillouin, por um determinado ângulo que vai de $\pm 1.15^{\circ}$ para a banda de condução e $\pm 1.55^{\circ}$ para a banda de valência. Quando o sistema é dopado com Sb a contribuição $S_{z}$ torna-se 

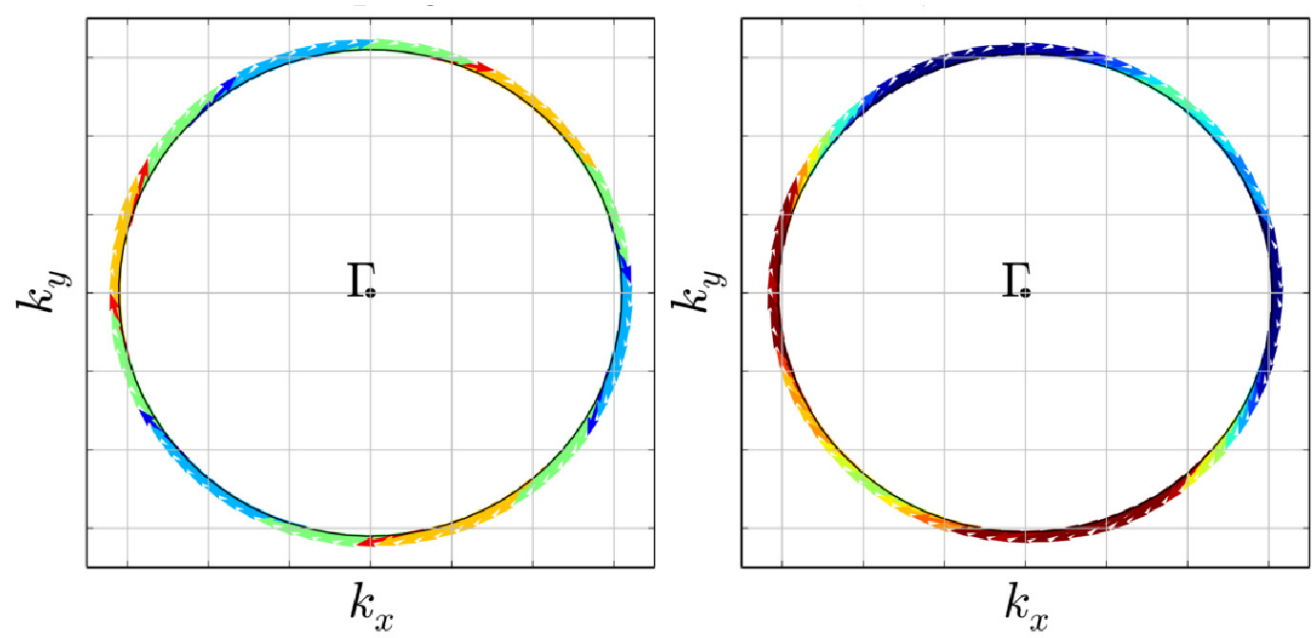

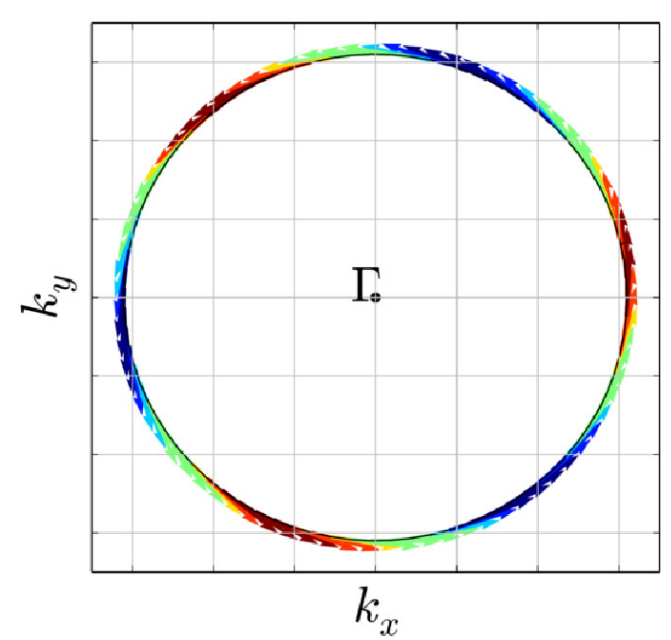

(a) Textura de spin de $\mathrm{Bi}_{2} \mathrm{Se}_{3}$.

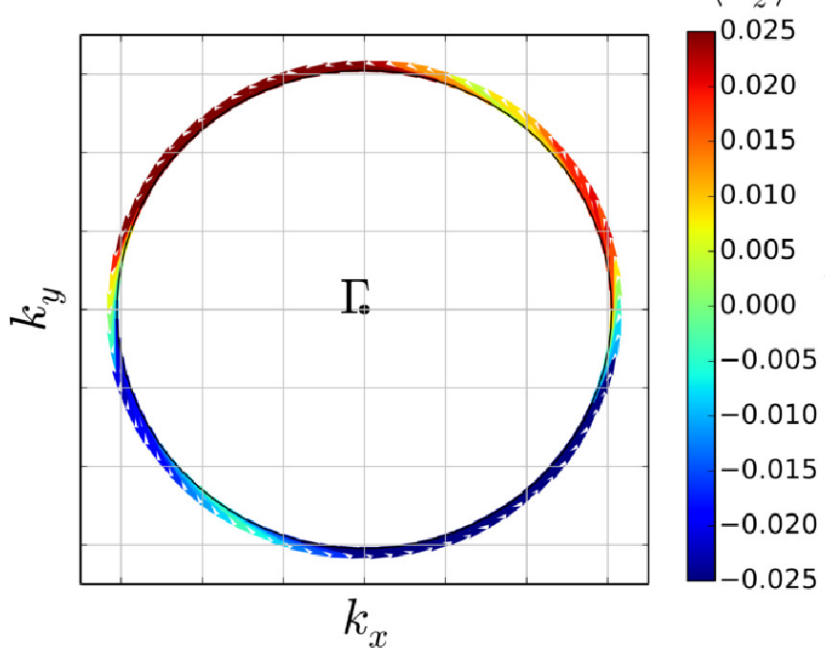

(b) Textura de spin de $\left(B i_{0.667} S b_{0.333}\right)_{2} \mathrm{Se}_{3}$.

Figura 43: Textura de spin para a parte superior (painel superior) e inferior (painel inferior) do cone de Dirac em (a) $\mathrm{Bi}_{2} S e_{3}$ e (b) (Bi $\left.i_{0.667} S b_{0.333}\right)_{2} S e_{3}$. A fatia de energia para obter a textura de spin foi de $0.1 \mathrm{eV}$ para a parte desocupada e -0.01 ( $\approx 0.0)$ eV para a parte ocupada de $\mathrm{Bi}_{2} \mathrm{Se}_{3}$ $\left(\left(B i_{0.667} S b_{0.333}\right)_{2} S e_{3}\right)$. A escala de cores representa o montante de $S_{z}$.

mais significativa, porém a oscilação tem menos nódulos. Para a concentração de $66 \%$ nas fatias de energia plotadas na figura 43 b vemos um vetor de spin $\vec{S}$ com ângulos em relação ao plano que variam de $\pm 1.60^{\circ}$ para a banda de condução e $\pm 5.38^{\circ}$ para a de valência. A textura de spin mostra claramente que a contribuição $S_{z}$ é mais pronunciada na banda de valência. A partir da figura observamos também que a simetria de reversão temporal é sempre preservada. 


\subsection{Conclusões parciais}

Neste trabalho, foi realizada uma detalhada e sistemática investigação das fases topológicas e das propriedades eletrônicas de superfície do $\left(\mathrm{Bi}_{x} \mathrm{Sb}_{1-x}\right)_{2} \mathrm{Se}_{3}$. A fim de fornecer uma descrição precisa da transição de fase trivial $\rightarrow$ TI , a liga foi descrita usando a abordagem SQS, e nós (i) examinamos a variação do gap em sua fase bulk, com base nos argumentos adiabáticos e de inversão de bandas, e (ii) calculou-se os invariantes topológicos $\left(\nu_{0}, \nu_{1} \nu_{2} \nu_{3}\right)$ para um conjunto de concentrações da liga. Em ambos os procedimentos, (i) e (ii), descobrimos que a transição trivial $\rightarrow$ TI ocorre para um concentração de $x=0.4$. Dentro da fase de TI, encontramos diferentes cenários da dispersão de energia de estados de superfície topologicamente protegidas dependendo da proporção entre Bi e Sb. Para a liga no intervalo de concentração de $0.4<x<0.7$, o DP fica acima dos estados ocupados da valência, sugerindo uma supressão de processos de retroespalhamento governado por estados de valência vindas do bulk. Esperamos que esses recursos possam corroborar para a compreensão de isolantes topológicos e pavimentar o caminho para sistemas mais complexos e aplicação em dispositivos de spintrônica reais. 



\section{Aumento do gap não trivial em Germaneno funcionalizado com Halogênios}

O efeito Hall quântico de spin (QSHE) proposto em 2005 por Kane e Mele para grafeno [4] e por S.-C. Zhang et al. em 2006 para o HgTe/CdTe [8], tornou-se uma área muito interessante da física da matéria condensada. Suas propriedades podem mudar o paradigma da nanoeletrônica, envolvendo transporte que flui sem dissipação de calor e possíveis aplicações em tecnologia, utilizando as propriedades dos chamados isolantes topológicos $[62,63,96]$. Nos materiais em que o efeito foi inicialmente previsto, o QSHE só foi verificado experimentalmente em heteroestruturas de $\mathrm{HgTe} / \mathrm{CdTe}$ [9]. No grafeno, devido ao acoplamento spin órbita fraco (SOC), a sua verificação experimental é problemática $[146,147]$. Além disso, na heteroestrutura de HgTe/CdTe a observação experimental é possível apenas em temperaturas muito baixas e controladas, uma vez que o gap devido a SOC neste material é de apenas $5 \mathrm{meV}[9]$.

Vários materiais têm sido propostos para superar o problema do pequeno gap SOC, como o material germaneno pristino (gap SOC de $29 \mathrm{meV}$ ) [148], o staneno (gap SOC de $100 \mathrm{meV}$ ) [22], e outros compostos binários com gaps que vão de meV até poucos eV [149,150]. Além dos materiais pristinos, também foi relatado que a funcionalização com halogênios nessas estruturas poderia aumentar o gap SOC $[22,24,151,152]$, como o staneno e germaneno funcionalizado com iodo apresentando um gap com SOC de 0.34 eV e $0.30 \mathrm{eV}$, tornando esses materiais bons candidatos, não só para a observação do 
QSHE à temperatura ambiente, mas para aplicações reais em dispositivos eletrônicos. Do ponto de vista experimental, apenas o germaneno foi recentemente sintetizado porém sua utilização na nanoeletrônica é ainda limitada [153].

Motivado por um trabalho experimental recente, onde o germanano (germaneno hidrogenado) foi isolado [23], Seixas et al. [154] propuseram uma maneira de observar o QSHE em germaneno, removendo os átomos de hidrogênio de uma forma padrão, criando uma nano estrada de germaneno dentro do germanano. Ao fazer isso, o germaneno, que é um isolante topológico, fica em contato com o germanano que por sua vez é um isolante trivial. Assim, estados de borda topologicamente protegidos aparecem na interface da nano estrada. Estes estados de interface são semelhantes aos apresentados pelas nanofitas de germaneno [155]. Neste tipo de situação, em que a estrutura do germaneno está completamente exposta a partir do germanano, alguns problemas poderiam estar presentes: (1) os átomos de hidrogênio se forem removidos em apenas um lado da estrada, podem dar origem ao magnetismo [156], degradando as propriedades topológicas do material; (2) o SOC do germaneno também é pequeno (29 meV), de modo que em temperaturas mais altas o efeito não será observado; (3) a região em que os átomos de hidrogênio foram removidos é muito reativa, sendo possível a adsorção de outros átomos que degradam as propriedades topológicas do sistema.

Nesta capítulo, vamos mostrar que, a substituição de um átomo de hidrogênio por um iodo em germanano cria um isolante topológico bidimensional com um gap SOC de $0.49 \mathrm{eV}$. Esta funcionalização abre novas rotas para a observação do efeito Hall quântico de spin. Mostramos também que estados topologicamente protegidos surgem em nano estradas na interface entre germanano e germaneno funcionalizado por iodo. Além disso, apresentamos que, mesmo com uma distribuição aleatória de iodo dentro germaneno, com concentração maior do que 58 \% o sistema ainda será um isolante topológico. 


\subsection{Metodologia}

Nossas simulações foram baseadas na teoria do funcional da densidade (DFT) [32,34] como implementado no código OpenMX $[55,157]$. Para o funcional de troca e correlação foi utilizada a aproximação GGA-PBE [46]. A interação spin órbita foi incluída via pseudopotenciais totalmente relativístico j-dependentes de norma conservada [158]. Tanto a constante de rede, bem como as posições atômicas foram relaxadas até que as forças residuais fossem menores do que $0.001 \mathrm{eV} / \AA$. Para calcular o invariante $Z_{2}$ do sistema foi utilizado o esquema proposto por Fu e Kane [63,66].

\subsection{Resultados}

Inicialmente estudamos um sistema composto por germaneno funcionalizado em ambos os lados por iodo $[24,151,152]$, bem como germaneno com hidrogênio em um lado e iodo no outro. O primeiro foi para aumentar o gap não trivial no germaneno e o último além de aumentar o gap devido a SOC, evita que o magnetismo apresentado pela funcionalização do hidrogênio na outra metade degrade as propriedades topológicas do material. Na figura 44a, temos uma representação esquemática dos sistemas considerados nas nossas simulações. O grupo funcional (átomos em laranja) podem ser H ou I. O gemanano (germaneno totalmente hidrogenado) é um semicondutor com um gap direto de 1.54 eV [23]. Quando ambos os átomos de hidrogênio são substituídos por iodo, o sistema aumenta a sua constante de rede de $4.06 \AA$ A para o GeH para $4.32 \AA$ para o Ge-I. Sem SOC o germaneno iodado é um semimetal e quando o SOC está incluído, há uma abertura de um gap não-trivial de $0.51 \mathrm{eV}$ no ponto $\Gamma$, como ilustrado na figura 44b. Além disso, quando hidrogênio é substituído por um de iodo de um lado inteiro do material, o comportamento nas propriedades estruturais é bastante semelhante ao sistema com dois átomos de iodo. A constante de rede do sistema é um pouco menor, em torno de $4.22 \AA$ e o gap não-trivial, é de cerca de $0.48 \mathrm{eV}$, como representado na figura 44c. Neste sistema obtivemos $Z_{2}=1$, sendo então um isolante topológico 2D.

Além do invariante $Z_{2}$, uma característica chave presente em um isolante topológico 

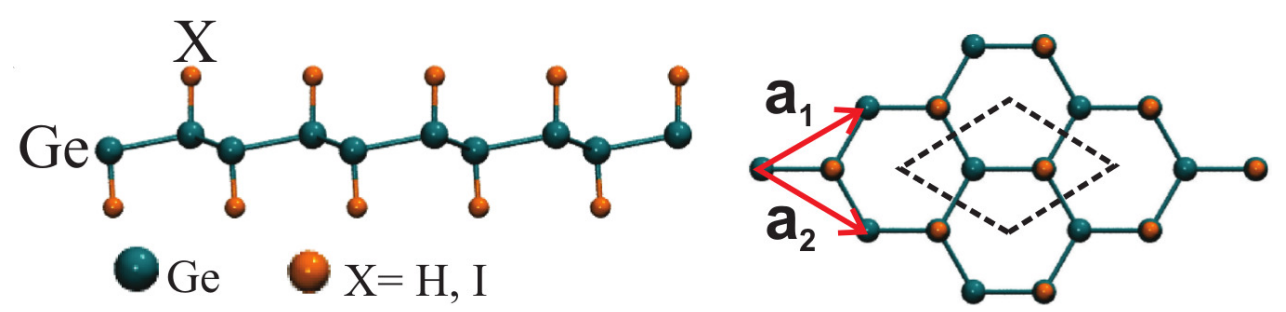

(a) Estrutura esquemática panorâmica e lateral de Ge-X.
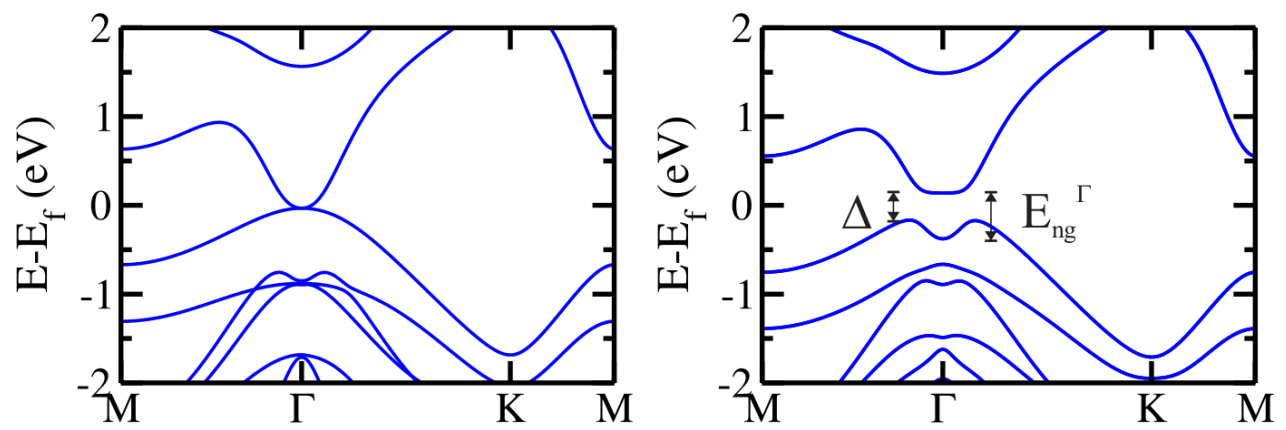

(b) Banda bulk de Ge-I com e sem SOC.
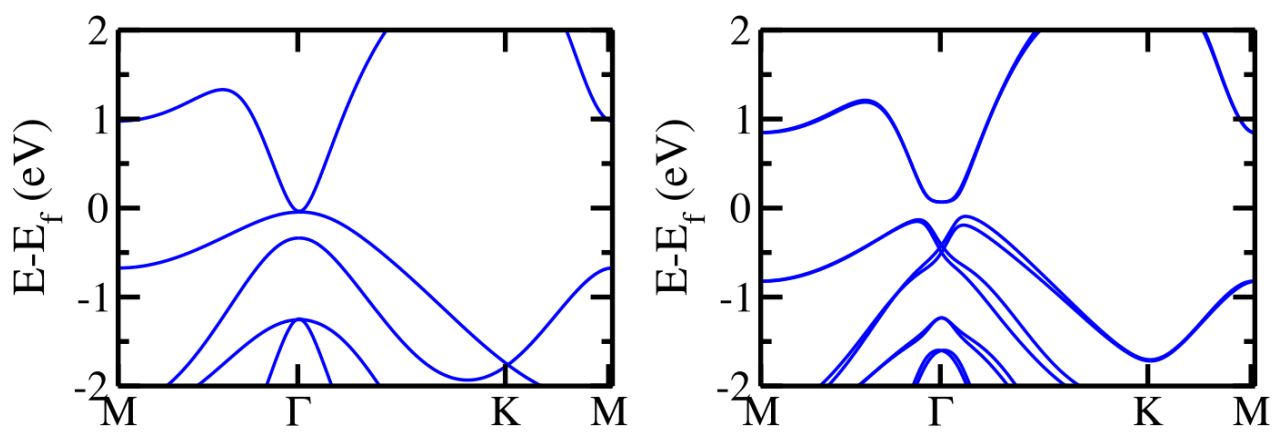

(c) Banda bulk de Ge-I-H com e sem SOC.

Figura 44: (a) Estrutura esquemática do germaneno funcionalizado, Ge-X, onde $X$ representa a funcionalização única por $H, I$ ou os compostos binários $I-H$. À esquerda (direita) temos a estrutura de banda para (b) Ge-I e (c) Ge-I-H, sem (com) acoplamento spin órbita. $E_{n g}^{\Gamma}$ e $\Delta$ representam o gap não-trivial que se abriu em $\Gamma$ pelo SOC e o gap indireto no sistema.

2D é a presença de estados de borda ou interface topologicamente protegidas quando o material é posto em contato com um isolante trivial. Estes estados topológicos são apresentados em um número ímpar de cones de Dirac. Em uma nanofita, estados de borda irão surgir pelo contato com o vácuo que é um isolante trivial. Na figura 45 mostramos a estrutura de banda e densidade de carga local em torno do nível de Fermi, para as nanofitas ZigZag e Armchair de Ge-I e Ge-I-H. Nesta situação, todos os átomos de hidrogênio em um ou ambos os lados são substituídos por iodo. Para o caso totalmente iodado, apresentado na figura 45a e 45b, para evitar qualquer interação entre as bordas, utilizou-se um tamanho de fita de $7.7 \mathrm{~nm}$ para a geometria Armchair e $6.1 \mathrm{~nm}$ para o caso ZigZag. 

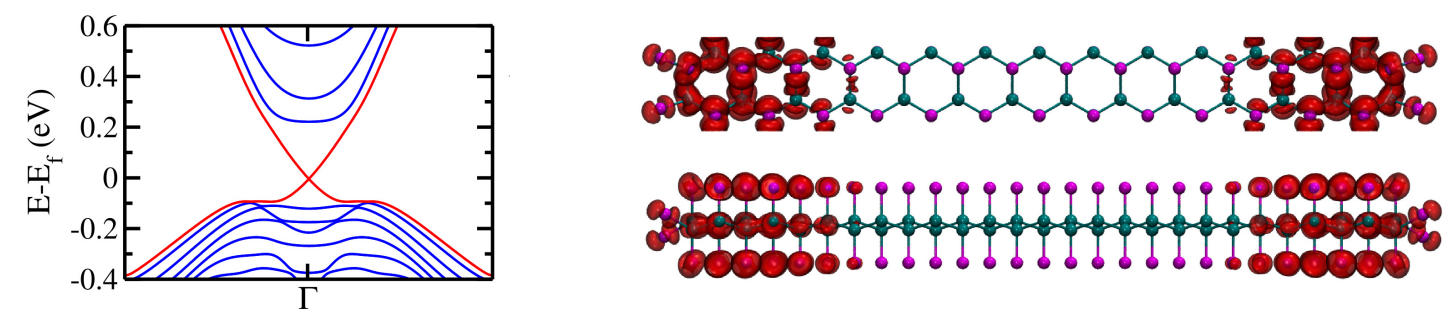

(a) Banda e densidade de carga local (Visão panorâmica e lateral) de Ge-I Armchair.
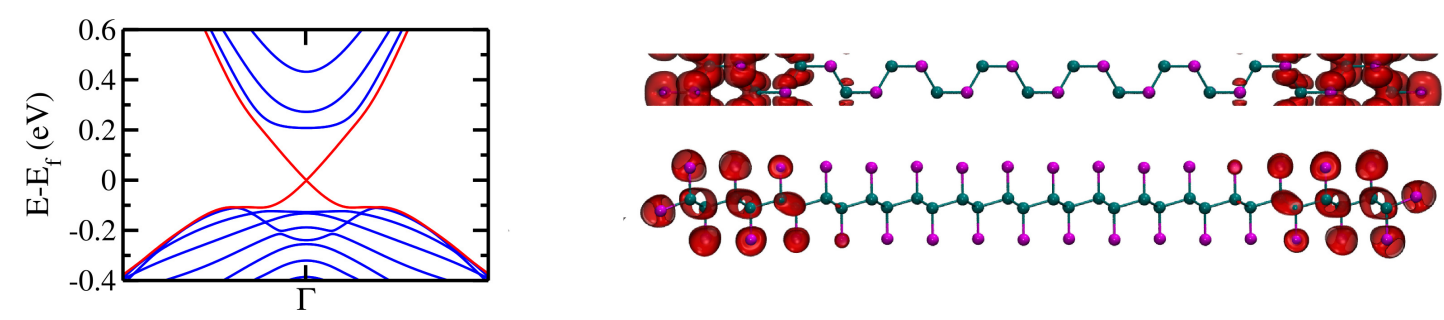

(b) Banda e densidade de carga local (Visão panorâmica e lateral) de Ge-I ZigZag.
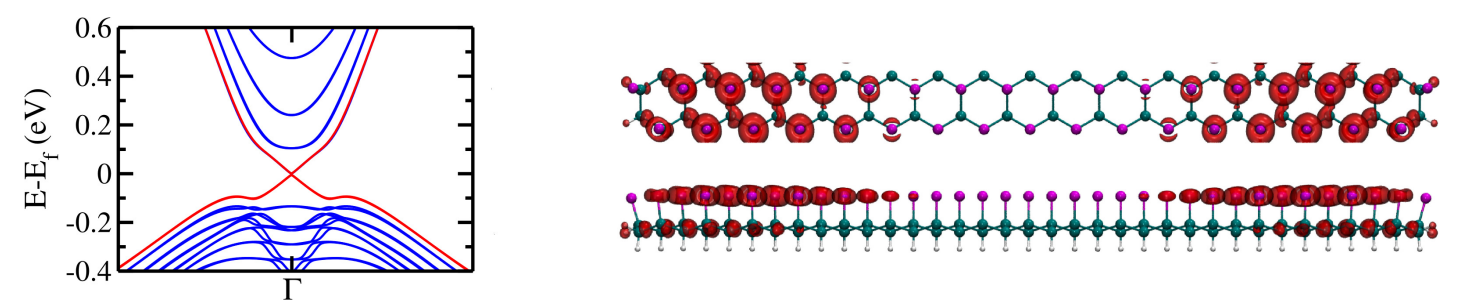

(c) Banda e densidade de carga local (Visão panorâmica e lateral) de Ge-I-H Armchair.
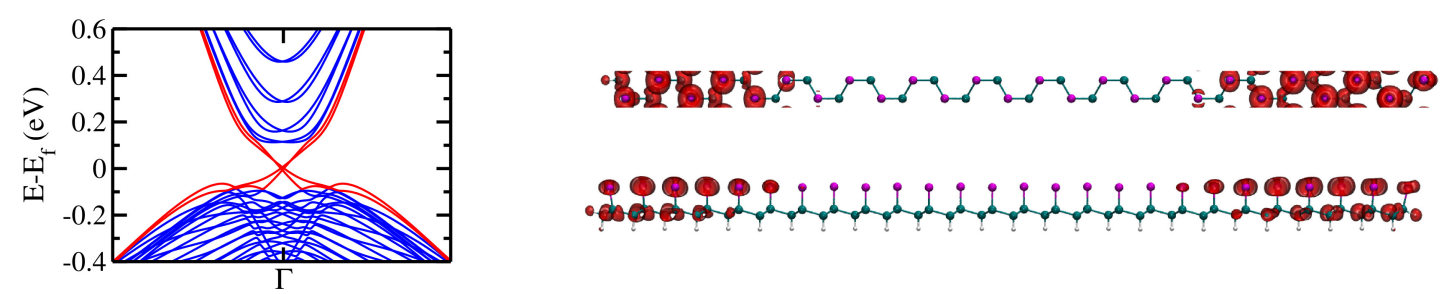

(d) Banda e densidade de carga local (Visão panorâmica e lateral) de Ge-I-H ZigZag.

Figura 45: À esquerda temos a estrutura banda e à direita a densidade de carga local, integrada em torno de 0,1 eV do nivel de Fermi para nanofitas (a) Armchair e (b) ZigZag de Ge-I e (c) Armchair e (d) ZigZag de Ge-I-H.

A partir deste resultado, podemos ver claramente a presença dos estados topológicos de borda (linhas vermelhas) na estrutura de banda, em que ambos os sistemas apresentam um cone de Dirac no ponto $\Gamma$. Também em cada figura 45a e 45b apresenta-se no painel da direita uma densidade de carga local, calculada em torno do nível de Fermi, que mostra que o cone de Dirac está localizado nas bordas do material. O mesmo comportamento foi observado para as nanofitas Armchair e ZigZag iodadas pela metade apresentados nas figuras 45c e 45d. O comportamento para as nanofitas Armchair em ambos os sistemas (Ge-I e Ge-I-H) são os mesmos, com os estados de borda sendo completamente 
degenerados. Para os casos ZigZag no sistema cuja metade esta iodada, há uma quebra de simetria, devido aos efeitos de relaxamento, levantando uma pequena degenerescência entre as bordas. Indo além da discussão sobre os estados de borda, que surgem devido

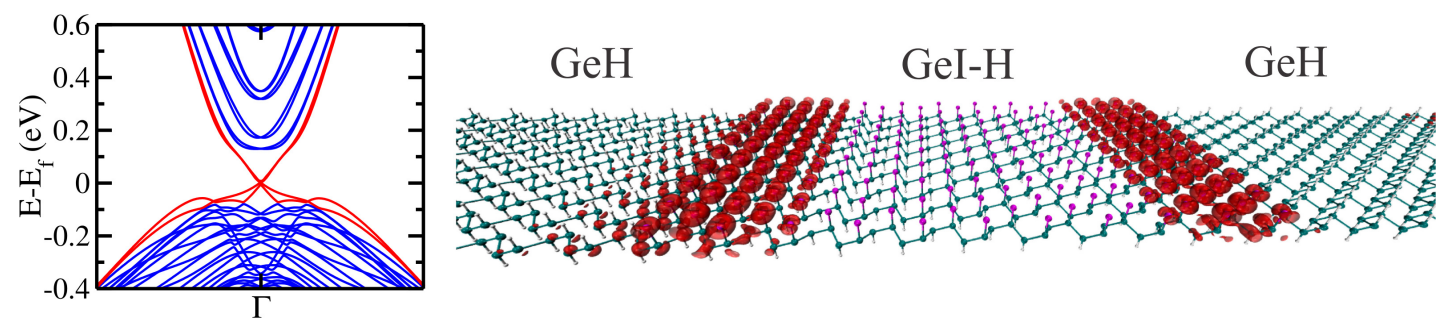

(a) Banda e densidade de carga local da nano estrada ZigZag de Ge-I-H.

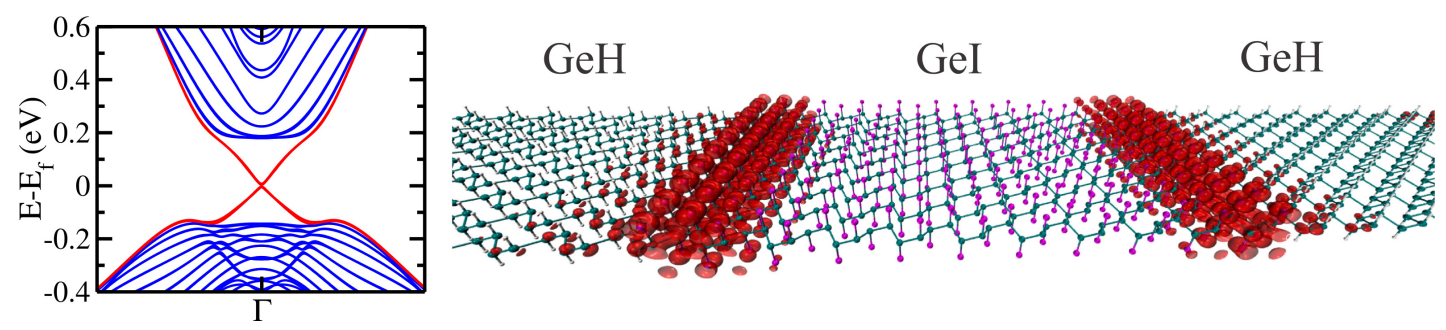

(b) Banda e densidade de carga local da nano estrada ZigZag de Ge-I.

Figura 46: À esquerda temos a estrutura de banda para uma nano estrada ZigZag e à direita a densidade de carga locais, integrado em torno de 0,1 eV do nível de Fermi para (a) Ge-I-H e (b) Ge-I.

ao limite de correspondência do bulk, quando um isolante topológico está ligado a um isolante trivial, podemos conectar as fitas consideradas nos resultados anteriores, criando o que chamamos nano estrada dentro do germanano $(\mathrm{Ge}-\mathrm{H})$. Vale a pena mencionar que este tipo de técnica para criar tais estradas também é usado em sistemas do tipo grafeno/grafano e grafeno/fluorografeno [159-161]. Representado na figura 46 apresentamos os resultados para a nano estrada criada dentro do germanene por um único (46a) ou ambos (46b) lados iodados. Mostramos apenas os resultados para o sistema ZigZag, pois o Armchair proporciona resultados semelhantes, como anteriormente demonstrado para as nanofitas. À esquerda, temos a estrutura da banda e na direita a densidade de carga local, integrado em torno de 0.1 eV o nível de Fermi. Como podemos ver, estados de Dirac topologicamente protegidos surgem na interface dos materiais, sendo espacialmente localizada na vizinhança da interface entre os materiais. É importante notar que, para a nanofita totalmente/parcialmente iodada ou para a nano estrada, independentemente da forma da borda ou interface (ZigZag ou Armchair) os estados topológicos de interface irão 
estar sempre presentes. Do ponto de vista experimental, a remoção completa de todos
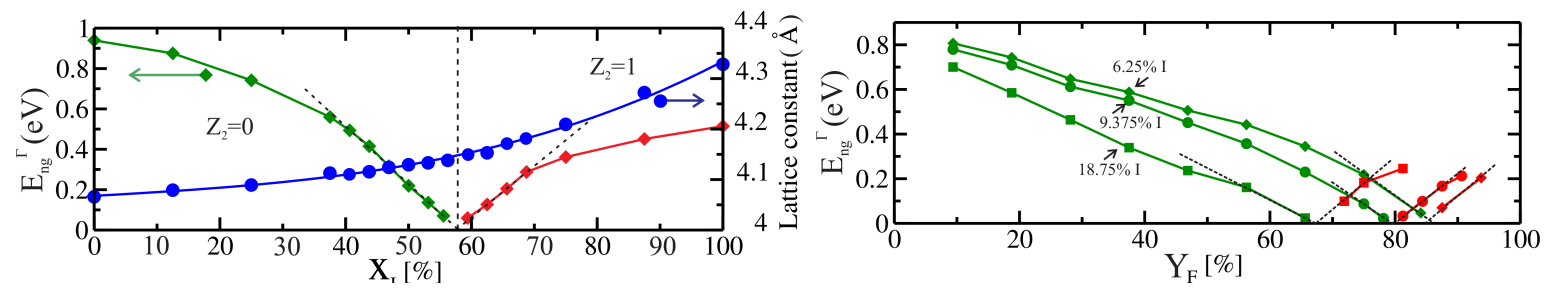

(a) Gap e parâmetro de rede em função de iodo.(b) Gap em função da concentração de flúor.

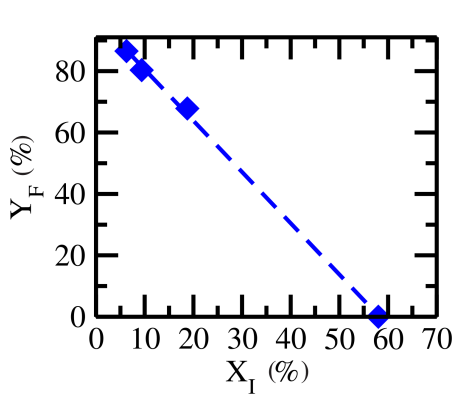

(c) Iodo em função de flúor na transição.

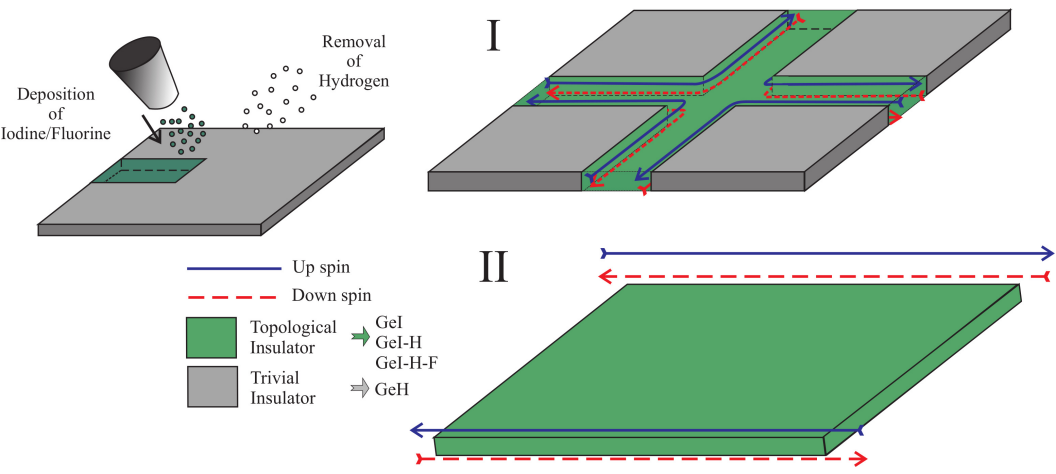

(d) Montagem experimental de uma nano estrada.

Figura 47: (a) Evolução do gap em função da concentração de iodo em Ge-H. (b) Evolução do gap da banda em função da concentração de flúor em Ge-H com diferentes concentrações de iodo. (c) A concentração de iodo como função da concentração de flúor no ponto de transição. (d) Vista esquemática de uma montagem experimental para criar uma nano estrada onde os átomos de hidrogênio são removidos e os átomos de iodo ou de flúor podem ser implantados para formar qualquer padrão desejado, como I e II.

os átomos de hidrogênio e consequente substituição por iodo, pode ser muito difícil, dependendo das condições experimentais. Numa situação de não-equilíbrio, alguns átomos de hidrogênio poderiam permanecer sobre o material. Desta forma é muito importante montar uma distribuição aleatória de átomos de iodo no padrão criado, de forma a ver se as propriedades topológicas do material permanecerão. Para este fim, foi feito um estudo da evolução das propriedades estruturais, eletrônicas e topológicas do germaneno submetidos a uma substituição aleatória do hidrogênio por iodo. Nós usamos uma supercélula composta por 32 átomos de germânio e de hidrogênio. Os átomos de hidrogênio foram substituídos por iodo em uma distribuição aleatória seguindo o procedimento SQS (Special Quasirandom Structure) [141], explicado no apêndice B. Tanto a estrutura atômica e os parâmetros de rede foram totalmente relaxados.

Na figura 47a mostramos a evolução da constante de rede e do gap no ponto $\Gamma$ do 
material em função da concentração de $\mathrm{X}_{\mathrm{I}}$ de iodo. Como podemos ver, a constante de rede em função da concentração não segue a lei de Vegard apresentando uma pequena curvatura. Este efeito é muito importante para as propriedades topológicas do sistema. Olhando para a evolução do gap da banda como função de $\mathrm{X}_{\mathrm{I}}$ determinou-se, utilizando tanto o argumento de continuidade adiabática, bem como pela avaliação $Z_{2}$, que o sistema sofre transição topológica em $\mathrm{X}_{\mathrm{I}} \approx 58 \%$, onde para concentrações até este valor o material é um isolante trivial e para valores maiores do que $58 \%$ o sistema é um isolante topológico 2D. É importante notar que quando o material tem uma distribuição aleatória de átomos de iodo, para $\mathrm{X}_{\mathrm{I}}=50 \%$ o sistema não é um isolante topológico, um cenário diferente para o sistema ordenado. Isto é devido ao parâmetro de rede menor nesta concentração, quando comparado com o sistema ordenado. Com o aumento na concentração de iodo a constante de rede do material aumenta e o sistema volta a ser um TI. Com base nestes resultados, mesmo se a nano estrada iodada não tem uma muito boa homogeneidade, se a concentração de átomos de iodo de uma certa região do material for maior do que 58 \% o sistema vai ser um isolante topológico 2D com bordas/interfaces topologicamente protegidas.

A técnica de funcionalização com halógenos é um método muito comum utilizado em sistemas do tipo grafeno, principalmente com o átomo de flúor [162-166]. Para o átomo de iodo, num sistema de grafeno, uma funcionalização completa do material não é uma técnica simples $[167,168]$. No caso do germaneno, como o sistema é muito mais reativo do que o grafeno, esta funcionalização poderia ser mais fácil, no entanto, é importante entender como podemos evitar a necessidade da utilização de uma grande concentração de átomos de iodo. Para este fim, estudamos os efeitos da presença de um átomo de flúor no sistema composto por ambos hidrogênio e iodo. Foram utilizados três concentrações de iodo em nossos cálculos: $\mathrm{X}_{\mathrm{I}}=6.250 \%, 9.375 \%$ e $18.750 \%$, que corresponde a 2,3 e 6 átomos de iodo em nossa supercélula. Essas concentrações foram distribuídas aleatoriamente na supercélula. Com as concentrações de iodo fixo, estudou-se a evolução da característica topológica do material como função da concentração de flúor, que também 
foram distribuídos aleatoriamente no material. Os resultados para o gap na banda é apresentado na figura 47b. Na figura 47c, dada uma concentração de flúor, vemos a concentração de iodo necessária para a transição topológica.

Finalmente, na figura 47d temos de forma esquemática a construção de um dispositivo para a observação do efeito Hall quântico de spin. Os átomos de hidrogênio podem ser removidos do sistema por alguma técnica experimental semelhante a erosão por plasma, e os átomos de iodo e flúor serão depositados nos sítios vazios. Este tipo de litografia poderia ser usado para construir padrões, como os apresentados se figura 47d I-II.

\subsection{Conclusões parciais}

Em conclusão, vimos que a subsituição dos átomos de hidrogênio por iodo leva o material a uma fase topológica, podendo chegar a gap não triviais de até $0.49 \mathrm{eV}$. Mostramos também que a criação de nano estradas com a funcionalização proporciona interfaces topologicamente protegidas. Em uma abordagem mais experimental notamos que uma distribuição aleatória de iodo dentro do sistema germaneno, com concentração maior do que $58 \%$ leva o material a sua fase topológica. Nota-se também que com uma funcionalização predominantemente de flúor sobre o hidrogênio, técnica já conhecida em materiais do tipo grafeno, apenas uma pequena quantidade de iodo é suficiente para uma transição topológica. Inclusive mostramos de forma esquemática a construção de um dispositivo para a observação desses estados topológicos protegidos de borda. Espera-se que esta funcionalização abra novas rotas para a observação do efeito Hall quântico de spin. 



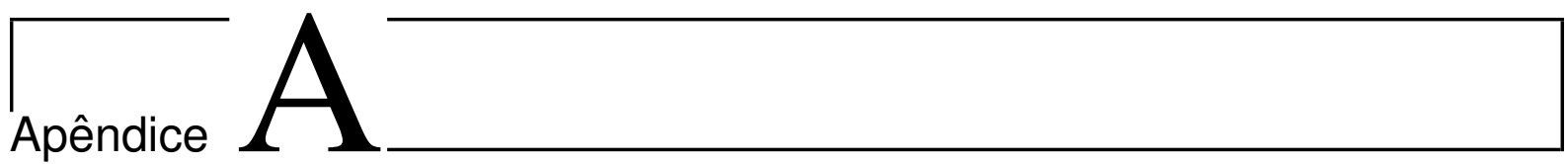

\section{Teoria Scanning Tunneling Microscope - STM}

O STM (Scanning Tunneling Microscope) foi o primeiro microscópio a ser desenvolvido na família de microscópios do tipo SPM (Scanning Probe Microscopy). A primeira imagem foi obtida em 1981 por G. Binnig e H. Rohrer na IBM de Zurich [169-173]. Em 1986 estes pesquisadores receberam o Prêmio Nobel de física por este invento. Um dos problemas mais fundamentais na física de superficie é a determinação de sua estrutura e este modo de operação permite a determinação da rede real do material em três dimensões, incluindo sistemas não-periódicos. Esta técnica é a de melhor resolução no SPM, permitindo propriamente a escala atômica.

\section{A.1 Como funciona um STM}

O STM é fundamentado a partir de 3 pilares:

1. Efeito Quântico de tunelamento: O STM utiliza uma ponta condutora muito fina, próxima à amostra $(\sim 10 \AA)$ condutora ou semicondutora, com uma diferença de pontencial aplicada $(\sim \mathrm{mV})$ entre elas, nesta situação ocorre passagem de corrente de tunelamento.

2. Piezoelétrica: Efeito descoberto por Pierre Curie em 1880 [174-176], comprimindo algum cristais (Quartz ou titanato de bário), o resultado é a criação de cargas na 
lateral do cristal, que pode ser revertido usando um potencial ao longo do material (controle de elongação e compressão).

3. Feedback computacional: Monitoramento das corrente de tunelamento e ajuste da ponta, para mapear a superfície de maneira a manter uma corrente constante ou uma altura constante.

Tudo isso fica mais claro na figura 48.

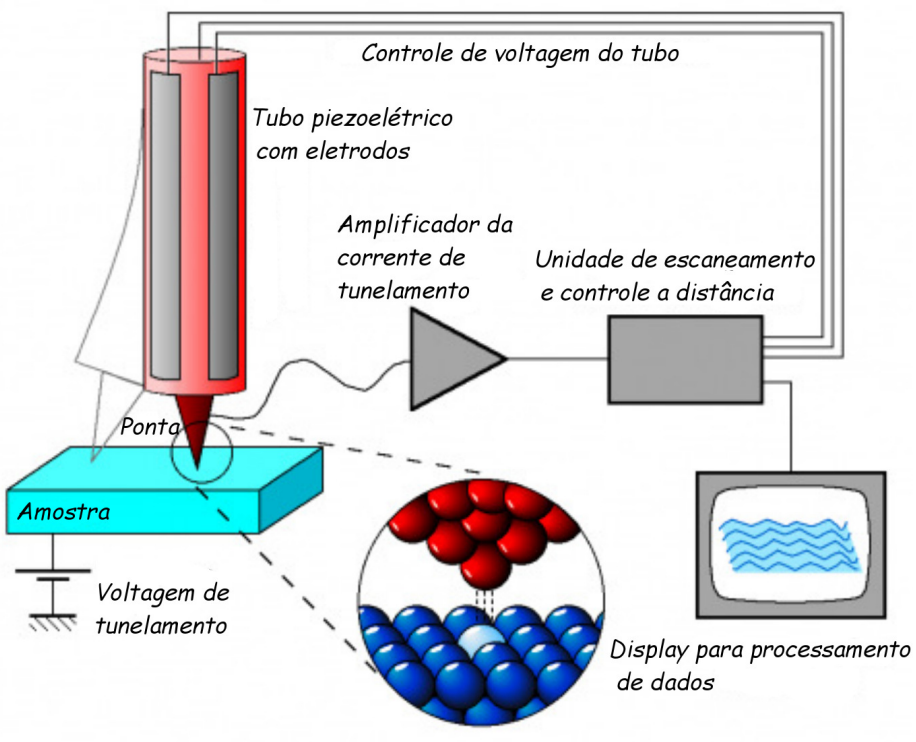

(a) Funcionamento de um STM.

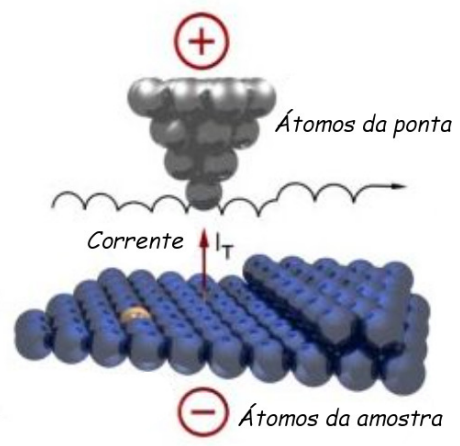

(b) Corrente de tunelamento.

Figura 48: Scanning Tunneling Microscope, em (a) temos o funcionamento de um STM e em (b) visualização da corrente tunelamento entre ponta e amostra.

\section{A.2 STM Ab Initio}

O STM pode ser modelado de maneira téorica, como descrito no artigo do J.Tersoff e D.R.Haman [120]. Estamos interessados na relação entre a corrente de tunelamento com os elementos que conhecemos das simulações téoricas.

A corrente de tunelamento em primeira ordem no formalismo de Bardeen [177] é dado pela equação:

$$
I=\frac{2 \pi e}{\hbar} \sum_{\mu, \nu} f\left(E_{\mu}\right)\left[1-f\left(E_{\nu}+e V\right)\right]\left|M_{\mu \nu}\right|^{2} \delta\left(E_{\mu}-E_{\nu}\right)
$$


onde $f(E)$ é a função de Fermi Dirac, $V$ a voltagem aplicada, $M_{\mu \nu}$ a matriz de tunelamento entre o estado $\psi_{\nu}$ da ponta e $\psi_{\mu}$ da superfície. Temos que perceber que $\psi_{\nu}$ e $\psi_{\mu}$ não são auto-estados da mesma hamiltoniana. No limite de baixas voltagens e baixas temperaturas ficamos com a equação:

$$
f\left(E_{\mu}\right)\left[1-f\left(E_{\nu}+e V\right)\right]=C \delta\left(E_{\nu}-E_{F}\right),
$$

onde $\mathrm{C}$ é um constante desconhecida. Integrando em todas as energias, obtemos $C \approx \mathrm{eV}$, logo ficamos com:

$$
I=\frac{2 \pi e^{2} V}{\hbar} \sum_{\mu, \nu}\left|M_{\mu \nu}\right|^{2} \delta\left(E_{\mu}-E_{F}\right) \delta\left(E_{\nu}-E_{F}\right) .
$$

Se fizermos o limite onde a ponta é substituida por um poço de potencial esférico pontual (medida não intrusiva da superfíce) os elementos da matriz ficam com a forma:

$$
I \propto \sum_{\nu}\left|\psi_{\nu}\left(\overrightarrow{r_{0}}\right)\right|^{2} \delta\left(E_{\nu}-E_{F}\right)=\rho\left(\vec{r}_{0}, E_{F}\right)
$$

onde $\vec{r}_{0}$ é a posição da ponta (pontual). Percebemos que a corrente é proporcional a LDOS na energia de Fermi, e também da posição da ponta. 

$\underset{\text { Apendice }}{B} \mathrm{~B}$

\section{Special Quasirandom Structure - SQS}

Cada material tem propriedades eletrônicas distintas, e dada a necessidade de se obter propriedades intermediárias entre certos sistemas criam-se ligas. Nas primeiras tentativas de descrever ligas, teorias estruturais eletrônicas tratavam estes materiais perturbativamente preservando as simetrias dos materiais.

Uma das aproximações mais conhecidas como VCA (Virtual Crystal Approximation) [178], cada sítio da liga $A_{1-x} B_{x}$ é uma mistura dos átomos $\mathrm{A}$ e $\mathrm{B}$ ou seja $\langle A B\rangle$, outra aproximação como SCPA (Site Coherent Potential Approximation) [179] átomos A e B tornam-se equivalentes porém inseridos num meio ou potencial uniforme. Nota-se que ambos os métodos são muitos restritivos:

- O VCA é limitado para métodos de estrutura eletrônica onde apenas elétrons de valência são considerados. É utilizado para uma variedade de materiais usando Hamiltonianos que incluam apenas a valência como método do pseudopotencial, ou método Tight Binding empírico.

- O SCPA é limitado para cálculos onde os elementos matriciais do potencial são associdados com sítios atômicos específicos. É utilizado em métodos do pseudopotencial empírico, $\vec{k} \cdot \vec{p}$ métodos perturbativos, método KKR, entre outros.

Além disso esses métodos são não estruturais, no sentido que rearranjos geométricos dos átomos não são considerados, e sabe-se da teoria de grupos que efeitos óticos, e eletrônicos estão associados com reduções de simetria. De fato uma das realizações mais 
importantes que emerge de cálculos ab initio de estrutura cristalinas, é que suas propriedades eletrônicas refletem os detalhes dos arranjos microscópicos dos átomos, incluindo pequenas mudanças como relaxações estruturais.

Recentemente Zunger et al $[141,180]$ desenvolveram um método, no qual estes problemas não existem, chamado de SQS (Special Quasirandom Structure). Neste método é construído uma célula unitária, cujos $\mathrm{M}$ sítios atômicos são ocupados por átomos $\mathrm{A}$ e B de forma específica, de maneira que a estrutura, como um todo, se assemelhe a uma configuração média de uma liga perfeitamente randômica $A_{1-x} B_{x}$.

Descrever ligas randômicas com estruturas periódicas introduz erros na função de correlação. Entretanto, dada uma supercélula grande o suficiente, e que interações entre vizinhos distantes contribuem eletronicamente menos do que as de primeiros vizinhos, a idéia do SQS é a reprodução da rede perfeitamente randômica para algumas camadas de vizinhos em torno de um sítio, jogando os erros de periodicidade para vizinhos que não sejam tão importantes. Podemos descrever o método da seguinte maneira:

Um certo arranjo dos atomos A e B, chamado de configuração $\sigma$, é discretizado em o que chamamos de figuras $f=(k, m)$, i.e para $k=2$ estamos discretizando em pares de átomos, para $m=3$, estamos considerando até terceiros vizinhos. Para cada sítio de figura definimos uma variável chamada pseudospin $S$ que pode assumir o valor 1 se o sítio é ocupado pelo átomo $\mathrm{A}$ ou -1 se é ocupado pelo átomo $\mathrm{B}$.

Assim define-se $\Pi_{f}(l, \sigma)=\Pi \hat{S}_{i}$ que é a soma das variáveis de pseudospin para um determinado sítio $l$ (onde $l$ inclui as várias maneiras que a figura pode se orientar naquele ponto da rede), de uma dada configuração $\sigma$. Lembrando que há $D_{f}$ figuras em cada sítio $l$ da rede, desta configuração obtemos o seguinte valor médio:

$$
\bar{\Pi}_{f}(\sigma)=\frac{1}{D_{f} N} \sum_{l} \Pi_{f}(l, \sigma) .
$$

A idéia de expansão em cluster é que a média de qualquer propriedade $\mathrm{P}$ pode ser obtida como segue:

$$
\langle P\rangle=\sum_{k, m} D_{k, m}\left\langle\bar{\Pi}_{k, m}\right\rangle p_{k, m}
$$


Aqui $p_{k, m}$ são parâmetros de interação da propriedade $\mathrm{P}$ e arranjo geométrico $f=(k, m)$. No limite de todas as figuras esta expansão é exata onde os produtos de pseudospin formam um conjunto completo.

Para uma liga binária perfeitamente randômica $A_{1-x} B_{x}$, temos que $\left\langle\Pi_{k, m}\right\rangle_{R}=(2 x-$ $1)^{k}$. Logo em vez de buscar $\left\langle\bar{\Pi}_{k, m}\right\rangle$, fazendo-se uma média sobre todas as possibilidades, construímos uma configuração especial cuja função de correlação $\left\langle\bar{\Pi}_{k, m}\right\rangle_{S}$ se aproxime ao máximo da configuração perfeitamente randômica, o que pode ser verificado via expressão:

$$
\delta \Pi_{k, m}(\sigma)=\left|\left\langle\bar{\Pi}_{k, m}\right\rangle_{S}-(2 x-1)^{k}\right| .
$$

A quantidade acima indica o quão aleatório a configuração especial S é, o método SQS gera um arranjo de átomos cujo desvio padrão em relação à função de correlação randômico é 0 até certo números de vizinhos $m_{\max }$ e até certa ordem de arranjos geométricos $k_{\max }$.

Para os cálculos de SQS, usaremos um programa criado no grupo, que considera apenas pares, ou seja $k=2$, o que é normalmente usado na literatura, e abordam-se apenas ligas binárias analogamente ao artigo publicado no grupo SAMPA (Simulação Aplicada a Materiais: Propriedades Atomísticas) [181]. O programa segue a seguinte metodologia:

1. Colocam-se randomicamente os átomos A e B em uma supercélula de N átomos com composição específica, cuja estrutura é determinada.

2. O programa calcula o desvio $\delta \Pi_{k, m}(\sigma)$, se o valor for maior que um valor predeterminado volta-se ao passo 1, caso contrário, encontramos a estrutura especial SQS.

3. Usando um programa de DFT, relaxa-se o material, e suas propriedades eletrônicas são calculadas. 



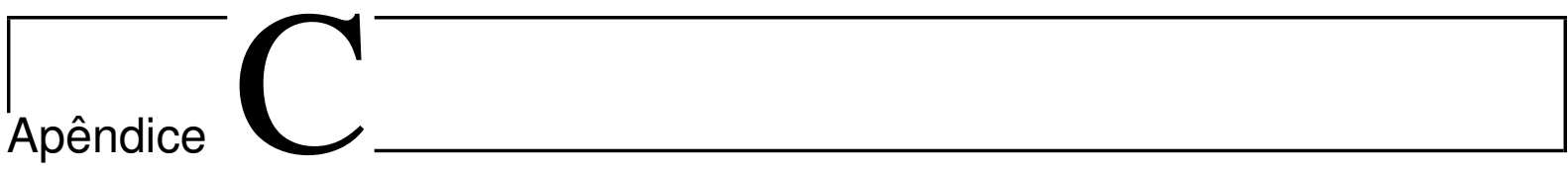

\section{Spin- and Angle- Resolved Photoemission Spectroscopy - ARPES and SARPES}

Sabemos que as propriedades macroscópicas de materiais sólidos são regidas pelas estruturas eletrônicas microscópicas. Logo é necessário estudar a bandas de dispersão do material de maneira a entender, controlar ou fazer algum uso prático das propriedades macroscópicas de sistemas avançados. Sabe-se que estados eletrônicos em materiais são o reflexo da energia do elétron $E$, momento cristalino $\vec{k}$ e seu spin $\vec{s}$. ARPES e SARPES são duas técnicas que relacionam estas 3 grandezas como veremos a seguir.

\section{C.1 Princípios básicos}

ARPES é o método para se estudar a estrutura eletrônica de sólidos usando o efeito fotoelétrico $[182,183]$. Um raio de luz monocromático de energia $h \nu$, proveniente de uma fonte de radiação sincrotrônica, incide na amostra e fotoexcita os elétrons para o vácuo. Este fotoelétrons são coletados em um analisador eletrostático que mede sua energia cinética em função dos angulos $(\vartheta, \varphi)$ relativos a superfície da amostra como visto na figura 49. Desta maneira o vetor de onda $\left(\vec{K}=\frac{\vec{p}}{\hbar}\right)$ dos fotoelétrons no vácuo são determinados via:

$$
\begin{aligned}
& K_{x}=\frac{1}{\hbar} \sqrt{2 m E_{k i n}} \sin (\vartheta) \cos (\varphi) \\
& K_{y}=\frac{1}{\hbar} \sqrt{2 m E_{k i n}} \sin (\vartheta) \sin (\varphi) \\
& K_{z}=\frac{1}{\hbar} \sqrt{2 m E_{k i n}} \cos (\vartheta) .
\end{aligned}
$$




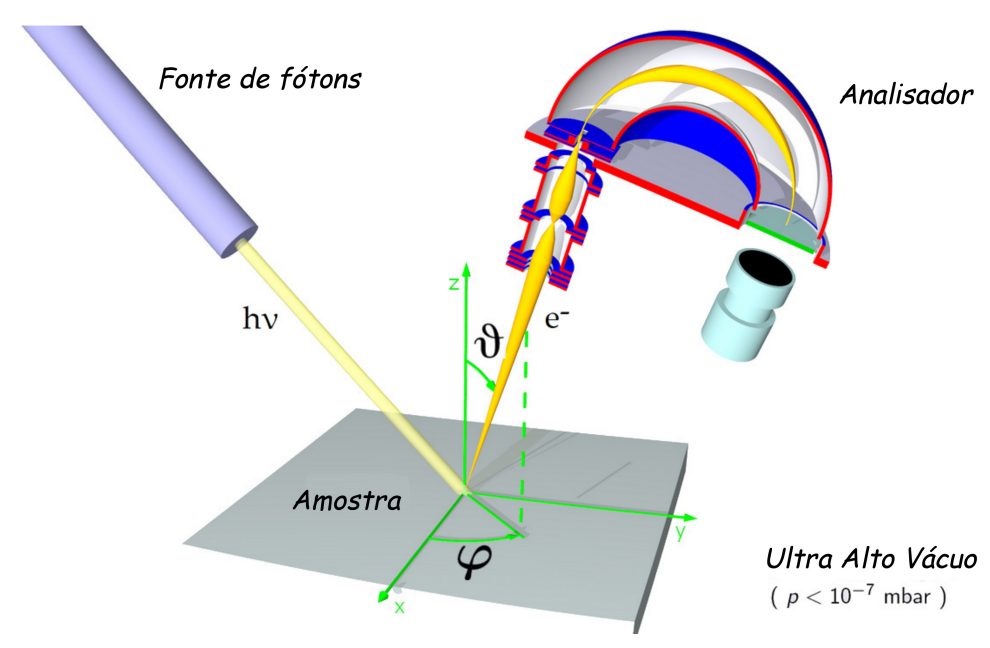

Figura 49: Geometria de um experimento de ARPES. A emissão de elétrons pela fonte é especificada pelos ângulos polar $\vartheta$ e azimutal $\varphi$, em seguida os fotoelétrons emitidos da amostra são coletados em um analisador eletrostático.

Pela conservação de energia podemos relacionar a energia cinética com a energia de ligação $E_{B}:$

$$
E_{k i n}=h \nu-W-\left|E_{B}\right|,
$$

onde $\mathrm{W}$ é a função trabalho do material. Sabe-se que devido à simetria translacional na superfície da amostra, a componente paralela do momento é conservada num processo de fotoemissão logo para $K_{\|}=\left(K_{x}, K_{y}, 0\right)$ temos:

$$
\left|K_{\|}\right|=\frac{1}{\hbar} \sqrt{2 m E_{k i n}} \sin (\vartheta) .
$$

Por outro lado, a componente do momento perpendicular $K_{\perp}$ não é conservada, porém pode ser obtida por métodos mais finos apesar da técnica ser mais utilizada para superfícies.

\section{C.1.1 Arpes com spin}

Devido ao acoplamento spin órbita, a distribuição do espalhamento de elétrons por núcleos pesados é anisotrópica, o que é chamado de espalhamento Mott. O design do detector de spin Mott é baseado neste efeito [184].

Para raios de elétrons com polarização $\vec{P}=\left(\frac{2}{\hbar}\right)\left(\left\langle S_{x}\right\rangle,\left\langle S_{y}\right\rangle,\left\langle S_{z}\right\rangle\right)$, a seção de choque é dada pela equação:

$$
\sigma(\Theta)=I(\Theta)[1+S(\Theta) \vec{P} \cdot \hat{n}]
$$


Com ângulo de espalhamento $\Theta$, intensidade de elétrons $I(\Theta)$, função de Sherman $S(\Theta)$ que é determinada pelo número atômico $Z$, ângulo de espalhamento e energia do raio incidente, e o vetor unitário $\hat{n}$ perpendicular ao plano de espalhamento definido por:

$$
\hat{n}=\frac{\vec{k}_{i} \times \vec{k}_{f}}{\left|\vec{k}_{i} \times \vec{k}_{f}\right|},
$$

onde $\vec{k}_{i}$ e $\vec{k}_{f}$ são vetores de onda dos elétrons iniciais e finais, e a direção $\hat{n}$ depende do fato do espalhamento ser para a direita ou esquerda. A medida da componente de polarização de spin acontece da seguinte maneira. Incide-se um raio de $\mathrm{N}$ elétrons com $N_{\uparrow}$ polarizados em $+\hat{z}$ e $N_{\downarrow}$ polarizados em $-\hat{z}$ cuja polarização é dada por $P_{z}=\frac{N_{\uparrow}-N_{\downarrow}}{N_{\uparrow}+N_{\downarrow}}$. Quando o espalhamento deste raio acontece devido a um núcleo no plano $x y$, haverá uma assimetria de espalhamento esquerda-direita $A_{z}(\Theta)$ vista na equação:

$$
A_{z}(\Theta)=\frac{N_{L}-N_{R}}{N_{L}+N_{R}}
$$

onde $N_{L}$ e $N_{R}$ são os números de elétrons espalhados para a direita ou esquerda através de um ângulo $\Theta$. Substituindo as relações $N_{L} \propto N_{\uparrow}[1+S(\Theta)]+N_{\downarrow}[1-S(\Theta)]$ e $N_{R} \propto$ $N_{\uparrow}[1-S(\Theta)]+N_{\downarrow}[1+S(\Theta)]$ obtemos a relação:

$$
P_{z}=\frac{A_{z}(\Theta)}{S(\Theta)}
$$

Esta última equação mostra que dada a função de Sherman, a medida de $A_{z}(\Theta)$ nos proporciona $P_{z}$. Em um polarímetro, é possível medir duas componentes de spin ortogonais de um raio de elétrons se arranjarmos quatro detetores em dois planos de espalhamento ortogonais de frente ao alvo. 



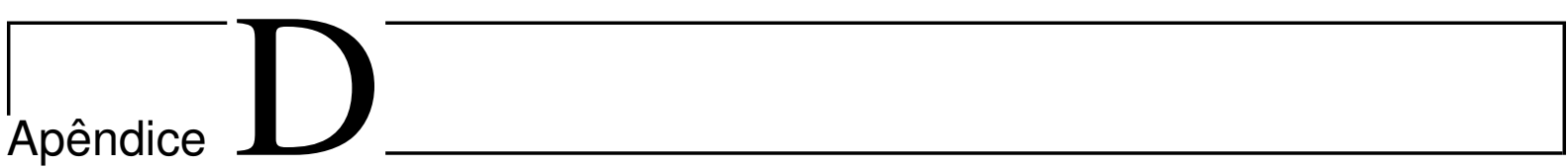

\section{Cálculo de $Z_{2}$ no formalismo de ondas planas}

A maneira mais simples de se saber se um isolante topológico é do tipo $Z_{2}$, é calculando seu invariante topológico. A formulação do invariante $Z_{2}$ é muito similar ao número de Chern visto no corpo da tese, o qual descreve o QHE pela teoria de polarização proposto por Resta [185]. Aqui a diferença é que a SOC terá o mesmo papel que o campo magnético no QHE. Antes de abordar os detalhes dos cálculos é necessário lembrar que existem algumas regras para que o $Z_{2}$ tenha sentido e possa ser calculado.

- O material tem que possuir TRS, caso contrário as operações matemáticas para elaboração do $Z_{2}$ não podem ser feitas. Logo, termos magnéticos na Hamiltoniana são proibidos.

- Devido a abordagem adiabática, no sentido que não pode haver mudanças bruscas da função de onda ao longo da zona de Brillouin, o material tem que possuir gap. Isto significa que um TI com gap em 3D (2D), apresentará um estado metálico topologicamente protegido na superfície 2D (borda 1D).

- É uma propriedade de bulk, ou seja todos os cálculos são feitos para apenas estados ocupados.

Nesta seção iremos mostrar duas maneiras diferentes de se calcular este invariante no formalismo de ondas planas. Iremos focar na avaliação do invariante, e não na física por trás deste que está descrita no corpo da tese. 


\section{D.1 Metodologia}

Para a avaliação do invariante $Z_{2}$ nosso cálculos foram baseados em DFT [32,34], usando o método do pseudopotencial, como PAW [59,60], para descrever as interações entre ion e elétron. A função de troca-correlação foi calculada na aproximação PBE [46] como implementado no código computacional VASP [30]. Os orbitais de Kohn-Sham foram expandidos em uma base de ondas planas com um corte em energia de 30\% superior ao valor máximo recomendado de forma a ter uma boa descrição da função de onda. A zona de Brillouin deve conter todos TRIM, ou seja pontos no qual existe um vetor da rede recíproca que leva ele a ele mesmo. Incluindo a SOC podemos escrever as funções de onda de Bloch como:

$$
\Psi_{n, \vec{k}}(\vec{r})=\left[\begin{array}{c}
\psi_{n, \vec{k}}^{\uparrow}(\vec{r}) \\
\psi_{n, \vec{k}}^{\downarrow}(\vec{r})
\end{array}\right],
$$

onde cada entrada tem o formato da equação:

$$
\psi_{n, \vec{k}}^{\sigma}(\vec{r})=\frac{1}{\sqrt{\Omega}} \sum_{j} C_{n, \vec{K}_{j}}^{\sigma} e^{i\left(\vec{k}+\vec{K}_{j}\right) \cdot \vec{r}} .
$$

Sendo $\Omega$ o volume da célula unitária, $C_{n, \vec{k}, \vec{K}_{j}}^{\sigma}$ são os coeficientes da expansão, $\sigma$ e $n$ são os índices de spin e banda, $\vec{k}$ é o ponto da rede recíproca e $\vec{K}_{j}$ é j-ésimo vetor da rede recíproca com $j$ um loop sobre todos os termos da expansão até $\left|\vec{k}+\vec{K}_{j}\right| \leq K_{\max }$ onde $K_{\text {max }}$ é o vetor de corte consistente com a energia de corte.

\section{D.2 Materiais 2D com simetria de inversão espacial}

Para meteriais 2D com simetria de inversão espacial usaremos o método proposto por Fu and Kane [67], onde o invariante topológico é dado pela equação:

$$
\begin{array}{ll}
(-1)^{Z_{2}} & =\prod_{T R I M} \delta_{a} \\
\delta_{a} & =\prod_{o c c}\langle P\rangle_{n}
\end{array}
$$

Onde o $Z_{2}$ pode ser 0 ou 1 , representando um isolante trivial ou topológico, $\langle P\rangle_{n}$ é o valor esperado do operador paridade para a band $n$, e occ significa que a produtória é feita sobre os pares de estados ocupados. No formalismo de ondas planas o método pode ser derivado como visto na equação (D.4). Note que como ambos os espinores possuem 
a mesma simetria de inversão espacial, o cálculo pode ser feito em apenas um deles. Por este motivo na equação (D.4) escondemos o componente de spinor $\sigma$.

$$
\begin{aligned}
\langle P\rangle_{n} & =\frac{\int d r \Psi_{n, \vec{k}}^{*}(\vec{r}) \hat{P} \Psi_{n, \vec{k}}(\vec{r})}{\int d r \Psi_{n, \vec{k}}^{*}(\vec{r}) \Psi_{n, \vec{k}}(\vec{r})}=\frac{\int d r \Psi_{n, \vec{k}}^{*}(\vec{r}) \Psi_{n, \vec{k}}(-\vec{r})}{\int d r \Psi_{n, \vec{k}}^{*}(\vec{r}) \Psi_{n, \vec{k}}(\vec{r})} \\
= & \frac{\int d r \sum_{K_{j}, K_{j}^{\prime}}\left(C_{n, \vec{K}_{j}}^{*} e^{-i\left(\vec{k}+\vec{K}_{j}\right) \cdot \vec{r}}\right)\left(C_{n, \vec{K}_{j}^{\prime}}^{*} e^{-i\left(\vec{k}+\vec{K}_{j}^{\prime}\right) \cdot \vec{r}}\right)}{\int d r \sum_{K_{j}, K_{j}^{\prime}}\left(C_{n, \vec{K}_{j}}^{*} e^{-i\left(\vec{k}+\vec{K}_{j}\right) \cdot \vec{r}}\right)\left(C_{n, \vec{K}_{j}^{\prime}}^{*} e^{i\left(\vec{k}+\vec{K}_{j}^{\prime}\right) \cdot \vec{r}}\right)} \\
= & \frac{\sum_{K_{j}} C_{n, \vec{K}_{j}}^{*} C_{n,\left(-2 \vec{k}-\vec{K}_{j}\right)}}{\sum_{K_{j}}\left|C_{n, \vec{K}_{j}}\right|^{2}}
\end{aligned}
$$

Logo nota-se que neste contexto o $Z_{2}$ torna-se uma soma de coeficientes.

\section{D.3 Materiais 2D sem simetria de inversão espacial}

Aqui usamos o método proposto por Fu e Kane [66] para materiais sem simetria de inversão espacial, na qual o invariante pode ser calculado da seguinte maneira:

$$
\begin{aligned}
(-1)^{Z_{2}} & =\prod_{T R I M} \delta_{\Lambda_{i}}, \\
\delta_{\Lambda_{i}} & =\frac{P f\left(\omega\left(\Lambda_{i}\right)\right)}{\sqrt{\operatorname{det} \omega\left(\Lambda_{i}\right)}}, \\
\omega_{n m}\left(\Lambda_{i}\right) & =\left\langle U_{n,-\Lambda_{i}}|\Theta| U_{m, \Lambda_{i}}\right\rangle=\int d \vec{r}\left\langle U_{n,-\Lambda_{i}} \mid \vec{r}\right\rangle \Theta\left\langle\vec{r} \mid U_{m, \Lambda_{i}}\right\rangle .
\end{aligned}
$$

Lembrando que o operador de TRS é dado por $\Theta=-i \sigma_{y} K$, onde $\sigma_{y}$ é a matriz de Pauli na direção $y$, e $K$ é um operador que retira o complexo conjugado. Usando a equação (D.6), podemos aplicar o TRS para obter $\Theta\left|U_{m, \Lambda_{i}}\right\rangle$ como visto na seguinte equação:

$$
\begin{aligned}
& \left\langle U_{n,-k} \mid \vec{r}\right\rangle= \\
& \frac{1}{\sqrt{\Omega}}\left(\sum_{\vec{K}_{j}} C_{n,-\vec{k}, \vec{K}_{j}}^{\uparrow *} e^{-i \vec{K}_{j} \cdot \vec{r}} \quad \sum_{\vec{K}_{j}} C_{n,-\vec{k}, \vec{K}_{j}}^{\downarrow *} e^{-i \vec{K}_{j} \cdot \vec{r}}\right) . \\
& \Theta\left\langle r \mid U_{m, k}\right\rangle=\frac{1}{\sqrt{\Omega}}\left(\begin{array}{c}
\sum_{\vec{K}_{j}}-C_{m, \vec{k}, \vec{K}_{j}}^{\downarrow *} e^{-i \vec{K}_{j} \cdot \vec{r}} \\
\sum_{\vec{K}_{j}}+C_{m, \vec{k}, \vec{K}_{j}}^{\uparrow *} e^{-i \vec{K}_{j} \cdot \vec{r}}
\end{array}\right) .
\end{aligned}
$$


Agora com estas operações, podemos calcular a matriz $\omega$ vista na equação (D.8).

$$
\begin{aligned}
\omega_{n m} & =\int d \vec{r}\left\langle U_{n,-\Lambda_{i}} \mid \vec{r}\right\rangle \Theta\left\langle r \mid U_{m, \Lambda_{i}}\right\rangle, \\
& =\frac{1}{\Omega} \int d \vec{r}\left(\begin{array}{c}
\sum_{\vec{K}_{j}, \vec{K}_{j^{\prime}}}-C_{n,-\Lambda_{i}, \vec{K}_{j}}^{\uparrow *} C_{m, \Lambda_{i}, \vec{K}_{j^{\prime}}}^{\downarrow *} e^{-i\left(\vec{K}_{j}+\vec{K}_{j^{\prime}}\right) \cdot \vec{r}} \\
\sum_{\vec{K}_{j}, \vec{K}_{j^{\prime}}} C_{n,-\Lambda_{i}, \vec{K}_{j}}^{\downarrow *} C_{m, \Lambda_{i}, \vec{K}_{j^{\prime}}}^{\uparrow *} e^{-i\left(\vec{K}_{j}+\vec{K}_{j^{\prime}}\right) \cdot \vec{r}}
\end{array}\right), \\
& =\sum_{\vec{K}_{j}}-C_{n, \Lambda_{i},-2 \Lambda_{i}-\vec{K}_{j}}^{\uparrow *} C_{m, \Lambda_{i}, \vec{K}_{j}}^{\downarrow *} \\
& +\sum_{\vec{K}_{j}} C_{n, \Lambda_{i},-2 \Lambda_{i}-\vec{K}_{j}}^{\downarrow *} C_{m, \Lambda_{i}, \vec{K}_{j}}^{\uparrow *}
\end{aligned}
$$

Onde nesta última operação fizemos $C_{n,-\Lambda_{i}, \vec{K}_{j}}^{\sigma}=C_{n, \Lambda_{i},-2 \Lambda_{i}-\vec{K}_{j}}^{\sigma}$, o que vale apenas nos TRIMs. Nesta metodologia o invariante pode também ser calculado como uma soma de coeficientes. Porém diferentemente do outro método o cálculo deve ser feito nos dois espinores.

\section{D.4 $Z_{2}$ em 3D}

Em sistemas 3D temos 8 TRIMs e existem 4 invariantes topológicos como vistos na figura 50. Nos TRIMs a dupla degenerescência de Kramer existe, i.e $\Theta H\left(\Lambda_{i}\right) \Theta^{-1}=H\left(\Lambda_{i}\right)$. Usando $\delta\left(\Lambda_{i}\right)$ em qualquer um dos métodos acima, podemos calcular os 4 invariantes chamados de $\left(\nu_{0}, \nu_{1} \nu_{2} \nu_{3}\right)$, como proposto na equação (D.9). Note que a robustez dos estados metálicos de superfície vem do invariante $\nu_{0}=1$.

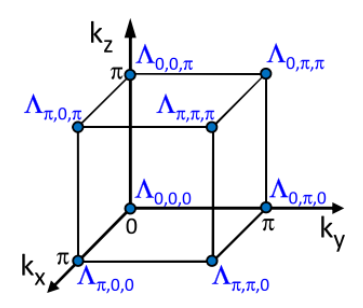

Figura 50: Extensão para 3D dos pontos TRIMs na zona de Brillouin. Imagem da referência [186].

$$
\begin{aligned}
& (-1)^{\nu_{0}}=\prod_{n_{j}=0, \pi} \delta\left(\Lambda_{n_{1}}, \Lambda_{n_{2}}, \Lambda_{n_{3}}\right) \\
& (-1)^{\nu_{i}}=\prod_{n_{j \neq i}=0, \pi ; n_{i}=\pi} \delta\left(\Lambda_{n_{1}}, \Lambda_{n_{2}}, \Lambda_{n_{3}}\right)(i=1,2,3) .
\end{aligned}
$$




\section{D.5 Implementação do $Z_{2}$}

Nesta tese elaboramos um programa escrito em linguagem python que calcula os invariantes topológicos em ambos os métodos. Para isso usamos um outro programa feito em fortran pelos professores R. M. Feenstra e M. Widom do departamento de física de Carnegie Mellon University, Pittsburgh, PA 15213. A versão preliminar do programa destes dois professores chamada de WaveTrans_spinor.f90 (modificada nesta tese), é livre na internet e calcula os coeficientes da função de onda do arquivo binário WAVECAR do pacote VASP. Além disso fizemos um outro programa que calcula o invariante por um outro método chamado de WCCs (Wanniers Charge Centers) no apêndice H. 



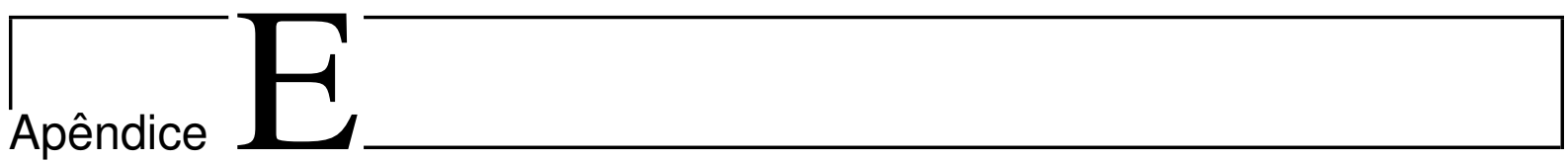

\section{Ausência de Retroespalhamento}

Nos isolantes topológicos do tipo $Z_{2}$, temos a presença do QSHE, neste efeito vemos que dois estados com spin opostos contrapropagam-se na superfície ou borda. Neste sistema próximo ao nível de Fermi há apenas o estado temporalmente revertido para espalhar (aqui sendo a diferença entre momentos $\vec{k}$ máxima, i.e $\vec{k}_{i} \rightarrow \vec{k}_{f}=-\vec{k}_{i}$, podemos falar de retroespalhamento, efeito que resulta na localização de Anderson na qual o sistema perde toda a condutividade para sistemas de baixa dimensão e alta desordem). Desta forma podemos demonstrar a ausência de retroespalhamento da seguinte forma: Dado um par de estados Kramer degenerados $\left|\phi_{i, \vec{k}, \uparrow}\right\rangle$ e $\left|\phi_{i,-\vec{k}, \downarrow}\right\rangle$, onde aqui foi descriminado a dependência de spin. Sabe-se que os estados estão relacionados pelo operador de simetria de reversão temporal por $\left|\phi_{i,-\vec{k}, \downarrow}\right\rangle=\Theta\left|\phi_{i, \vec{k}, \uparrow}\right\rangle$. Logo como o operador é antiunitário, ficamos com a equação (E.1).

$$
\begin{aligned}
\left\langle\phi_{i,-\vec{k}, \downarrow}|U| \phi_{i, \vec{k}, \uparrow}\right\rangle & =-\left\langle\phi_{i,-\vec{k}, \downarrow}\left|U \Theta^{2}\right| \phi_{i, \vec{k}, \uparrow}\right\rangle \\
& =-\left\langle\phi_{i,-\vec{k}, \downarrow}\left|\Theta \Theta^{-1} U \Theta \Theta\right| \phi_{i, \vec{k}, \uparrow}\right\rangle \\
& =-\left\langle\phi_{i, \vec{k}, \uparrow}|U| \phi_{i,-\vec{k}, \downarrow}\right\rangle^{*} \\
& =-\left\langle\phi_{i,-\vec{k}, \downarrow}|U| \phi_{i, \vec{k}, \uparrow}\right\rangle .
\end{aligned}
$$

Dado que $U$ é um operador invariante sob reversão temporal, e usando a propriedade que $\Theta^{2}=-1$, obtemos que a probabilidade de ocorrer uma transição de pares degenerados de Kramer em um sistema com simetria de reversão temporal é nula. 



\section{${ }_{\text {Aponadice }} \mathrm{F}$}

\section{Matemática da matriz $\omega$}

Aqui iremos discutir a matemática da matriz omega, e os truques para usá-la de forma adequada. A matriz é escrita da seguinte forma:

$$
\omega_{\alpha, \beta}(\vec{k})=\left\langle u_{\alpha,-\vec{k}}|\Theta| u_{\beta, \vec{k}}\right\rangle .
$$

Onde $\alpha$ e $\beta$ são índices de banda. A matriz que relaciona estes dois autoestados de Bloch é dada via:

$$
\begin{aligned}
\left|u_{\alpha,-\vec{k}}\right\rangle & =\sum_{\beta} \omega_{\alpha, \beta}^{*}(\vec{k}) \Theta\left|u_{\beta, \vec{k}}\right\rangle, \\
& =\sum_{\beta, l}\left\langle u_{\alpha,-\vec{k}}^{*}|\Theta| u_{\beta, \vec{k}}^{*}\right\rangle\left\langle u_{l,-\vec{k}}^{*}|\Theta| u_{\beta, \vec{k}}\right\rangle\left|u_{l,-\vec{k}}\right\rangle, \\
& =-\sum_{\beta, l}\left\langle u_{l,-\vec{k}}^{*}|\Theta| u_{\beta, \vec{k}}\right\rangle\left\langle u_{\beta, \vec{k}}^{*}|\Theta| u_{\alpha,-\vec{k}}^{*}\right\rangle\left|u_{l,-\vec{k}}\right\rangle, \\
& =-\sum_{l}\left\langle u_{l,-\vec{k}}^{*}\left|\Theta^{2}\right| u_{\alpha,-\vec{k}}^{*}\right\rangle\left|u_{l,-\vec{k}}\right\rangle, \\
& =\sum_{l}\left\langle u_{l,-\vec{k}}^{*} \mid u_{\alpha,-\vec{k}}^{*}\right\rangle\left|u_{l,-\vec{k}}\right\rangle, \\
& =\left|u_{\alpha,-\vec{k}}\right\rangle .
\end{aligned}
$$

Sabendo disso queremos mostrar que a matriz omega é unitária, para isso vamos calcular a seguinte expressão:

$$
\begin{aligned}
\sum_{\alpha} \omega_{\gamma, \alpha}(\vec{k})^{\dagger} \omega_{\alpha, \beta}(\vec{k}) & =\sum_{\alpha} \omega_{\alpha, \gamma}(\vec{k})^{*} \omega_{\alpha, \beta}(\vec{k}), \\
& =\sum_{\alpha}\left\langle u_{\alpha,-\vec{k}}^{*}|\Theta| u_{\gamma, \vec{k}}^{*}\right\rangle\left\langle u_{\alpha,-\vec{k}}|\Theta| u_{\beta, \vec{k}}\right\rangle, \\
& =-\sum_{\alpha}\left\langle u_{\beta, \vec{k}}|\Theta| u_{\alpha,-\vec{k}}\right\rangle\left\langle u_{\alpha,-\vec{k}}^{*}|\Theta| u_{\gamma, \vec{k}}^{*}\right\rangle \\
& =\left\langle u_{\beta, \vec{k}}|\Theta| u_{\gamma, \vec{k}}^{*}\right\rangle \\
& =\left\langle u_{\beta, \vec{k}}^{*}|\Theta| u_{\gamma, \vec{k}}\right\rangle, \\
& =\delta_{\beta, \gamma} .
\end{aligned}
$$

Note que a matriz tem a seguinte propriedade:

$$
\omega_{\beta, \alpha}(-\vec{k})=\left\langle u_{\beta, \vec{k}}|\Theta| u_{\alpha,-\vec{k}}\right\rangle=-\left\langle u_{\alpha,-\vec{k}}|\Theta| u_{\beta, \vec{k}}\right\rangle=-\omega_{\alpha, \beta}(\vec{k}) .
$$


Nos pontos TRIMs obtemos:

$$
\omega_{\beta, \alpha}\left(\vec{\Lambda}_{i}\right)=-\omega_{\alpha, \beta}\left(\vec{\Lambda}_{i}\right)
$$

Desta forma para um sistema de duas bandas ocupadas podemos escrever a matriz $\omega$ como um bloco $2 \times 2$ de forma que num ponto TRIM ela toma o seguinte formato:

$$
\omega\left(\vec{\Lambda}_{i}\right)=\left(\begin{array}{cc}
0 & \omega_{12}\left(\vec{\Lambda}_{i}\right) \\
-\omega_{12}\left(\vec{\Lambda}_{i}\right) & 0
\end{array}\right)=\omega_{12}\left(\vec{\Lambda}_{i}\right)\left(\begin{array}{cc}
0 & 1 \\
-1 & 0
\end{array}\right) .
$$

Outra matriz conveniente para sistema com simetria de reversão temporal é a matriz de conexão de Berry:

$$
\vec{a}_{\alpha, \beta}(\vec{k})=-i\left\langle u_{\alpha, \vec{k}}\left|\nabla_{\vec{k}}\right| u_{\beta, \vec{k}}\right\rangle
$$

Esta matriz tem algumas propriedades. Se relacionamos a matriz $\vec{a}_{\alpha, \beta}(\vec{k}) \operatorname{com} \vec{a}_{\alpha, \beta}(-\vec{k})$ podemos escrever o seguinte:

$$
\begin{aligned}
& \vec{a}_{\alpha, \beta}(-\vec{k})=-i\left\langle u_{\alpha,-\vec{k}}\left|\nabla_{-\vec{k}}\right| u_{\beta,-\vec{k}}\right\rangle \\
&=-i \sum_{\gamma}\left\langle u_{\alpha,-\vec{k}}\right| \nabla_{-\vec{k}}\left(\omega_{\alpha, \gamma}^{*}(\vec{k}) \Theta\left|u_{\gamma, \vec{k}}\right\rangle\right) \\
&=-i \sum_{\gamma}\left\langle u_{\alpha,-\vec{k}}|\Theta| u_{\gamma, \vec{k}}\right\rangle \nabla_{-\vec{k}} \omega_{\alpha, \gamma}^{*}(\vec{k}) \\
&-i \sum_{\gamma}\left\langle u_{\alpha,-\vec{k}}\left|\Theta \nabla_{-\vec{k}}\right| u_{\gamma, \vec{k}}\right\rangle \omega_{\alpha, \gamma}^{*}(\vec{k}), \\
&=-i \sum_{\gamma} \omega_{\alpha, \gamma}(\vec{k}) \nabla_{-\vec{k}} \omega_{\alpha, \gamma}^{*}(\vec{k}), \\
&-i \sum_{\gamma, l}\left\langle u_{\alpha,-\vec{k}}|\Theta| u_{l, \vec{k}}\right\rangle\left\langle u_{l, \vec{k}}\left|\nabla_{-\vec{k}}\right| u_{\gamma, \vec{k}}\right\rangle \omega_{\alpha, \gamma}^{*}(\vec{k}), \\
& \vec{a}_{\alpha, \beta}(\vec{k}) \quad=-i \sum_{\gamma} \omega_{\alpha, \gamma}(-\vec{k}) \nabla_{\vec{k}} \omega_{\alpha, \gamma}^{*}(-\vec{k})+\sum_{\gamma} \omega_{\alpha, l}(-\vec{k}) a_{l, \gamma}(-\vec{k}) \omega_{\alpha, \gamma}^{*}(-\vec{k}) .
\end{aligned}
$$

Note que no último passo trocamos $\vec{k} \rightarrow-\vec{k}$, agora retirando a notação matricia ficamos com:

$$
\vec{a}(\vec{k})=\omega(\vec{k}) \vec{a}(-\vec{k}) \omega^{\dagger}-i \omega(\vec{k}) \nabla_{\vec{k}} \omega^{\dagger}
$$

Tirando o traço de $\vec{a}(\vec{k})$ obtemos:

$$
\begin{aligned}
& \operatorname{Tr}[\vec{a}(\vec{k})]=\operatorname{Tr}[\vec{a}(-\vec{k})]-i \operatorname{Tr}\left[\omega \nabla_{\vec{k}} \omega^{\dagger}\right], \\
& \operatorname{Tr}[\vec{a}(\vec{k})]=\operatorname{Tr}\left[\vec{a}(-\vec{k})+i \operatorname{Tr}\left[\omega^{\dagger} \nabla_{\vec{k}} \omega\right] .\right.
\end{aligned}
$$

Onde usamos o fato que $\operatorname{Tr}\left[\nabla_{\vec{k}}\left(\omega^{\dagger} \omega\right)\right]=\operatorname{Tr}\left[\nabla_{\vec{k}} 1\right]=0$. Logo $\omega^{\dagger} \nabla_{\vec{k}} \omega=-\omega \nabla_{\vec{k}} \omega^{\dagger}$. Com essas ferramentas podemos calcular o invariante topológico $Z_{2}$. 


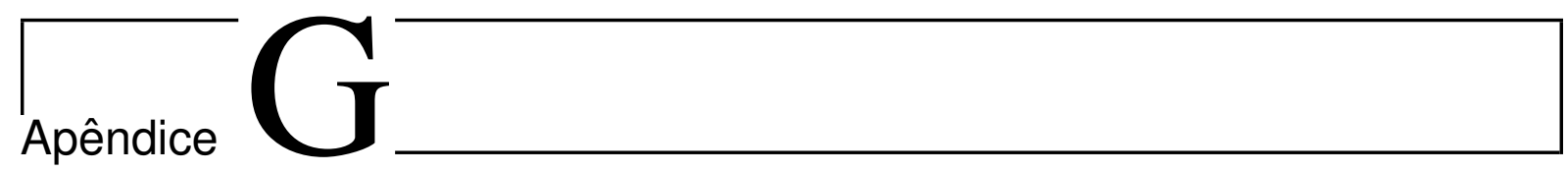

\section{PROGRAMA AWIREK}

O programa AwireK foi desenvolvido por Leonardo Abdalla e Alexsandro Kirch, originalmente para a geração de geometrias de nanofios estruturados. Posteriormente foram agregadas outras funcionalidades para a criação e tratamento de geometrias. Em 11.08.14 foi protocolado junto ao Instituto Nacional da Propriedade Industrial - I.N.P.I./S.P. o registro do pedido de registro de programa de computador de sob o título "AWIREK", o qual recebeu o noํ. BR 512014 000889-4. Na figura 51 é apresentada a interface gráfica do programa.

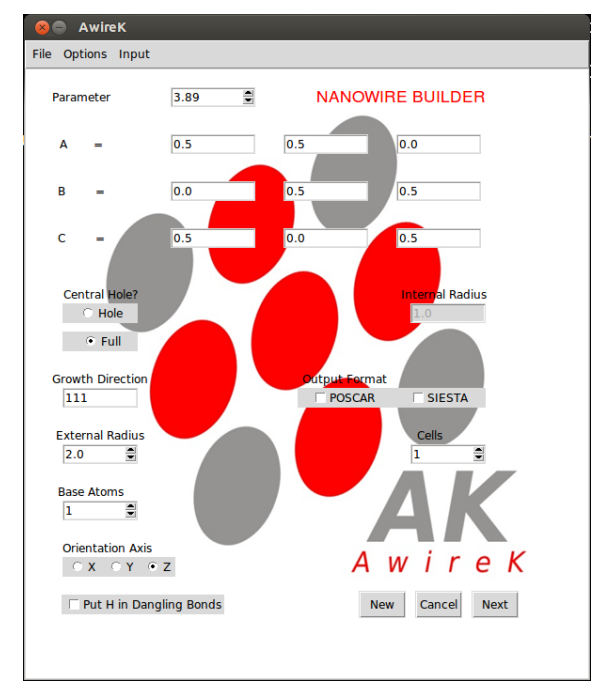

Figura 51: Interface gráfica do AwireK.

A criação dos nanofios está baseada em cortes no bulk do material, em uma determinada direção de crescimento em relação aos planos de Miller. Parâmetros tais como diâmetro, comprimento e direção de crescimento podem ser configurados no programa. 



\section{Método $Z_{2}$ no formalismo de Wannier Charge Centers - WCCs}

Neste apêndice gostaríamos de abordar um outro método para o cálculo de $Z_{2}$. Como este invariante involve a conexão de Berry, um cálculo não muito preciso envolveria uma correção no calibre, tarefa difícil para cálculos de primeiros princípios. Durante a tese vimos que existe muitas maneiras de se mapear se um sistema é isolante topológico ou não. Porém cada um deles tem suas vantagens e desvantagens que veremos a seguir.

1. A primeira maneira e mais óbvia seria o cálculo de superfície ou bordas para observar os estados metálicos. Em uma simulação computacional haveria a necessidade de se colocar vácuo devido as condições de Born-von Karman, e com isso seria possível observar os estados metálicos topologicamente protegidos de superfície. O problema deste método é o custo computacional pois, além de se colocar vácuo, não sabemos a quantidade necessária para evitar uma possível interação entre bordas ou superfícies (da mesma supercélula ou de células distintas).

2. O argumento de continuidade adiabática. Ele afirma que, se um hamiltoniano de um sistema é adiabaticamente transformado em outro sem fechar o gap e sem mudar as simetrias orginais [144], o sistema deve compartilhar a mesma classe topológica. Por outro lado, durante esta transformação, se o intervalo da banda de energia fecha, ocorrerá um mudança de fase topológica. Este método é muito bom para se estudar 
ligas ou transições de materiais nas quais não há mudança de simetria. Porém existe a necessidade de conhecimento prévio da topologia dos extremos da liga.

3. Inversão de bandas. Neste argumento baseado em teoria de grupos, quando ocorre uma inversão de bandas ao se incluir correções relativísticas de spin órbita o material necessariamente é um isolante topológico. Porém se o material sofre forte hibridização cada estado torna-se um mistura de outros, dificultando a visualização de tal inversão.

4. Calcular o Invariante $Z_{2}$ via paridade. Neste método se calcula o invariante $Z_{2}$ via autovalores do operador paridade, apesar de ser um método muito confiável ele apenas funciona para materiais que possuam a simetria de inversão espacial.

5. Método do Pffafiano sem simetria de inversão. Este método calcula o invariante $Z_{2}$ por um método descrito em apêndices anteriores da tese. O método requer uma base muito bem descrita. O problema deste invariante é que sua formulação é simples apenas em ondas planas. Para sistemas grandes na qual uma abordagem de base localizada é mais apropriada, sua formulação fica complexa.

Por estes motivos, decidimos calcular também o $Z_{2}$ pela troca de pares das funções de Wannier, durante um ciclo de reversão temporal descrito nas referências $[187,188]$.

\section{H.1 Formalismo teórico}

Sabemos que o invariante topológico $Z_{2}$ pode ser escrito como:

$$
Z_{2}=P_{\Theta}(t=T / 2)-P_{\Theta}(t=0),
$$

onde $t$ representa um tempo adiabático. Vamos agora reescrever a equação (H.1), em termos dos centros de carga de Wanniers (WCCs - Wanniers Charge Centers). As funções de Wanniers (WFs) pertencentes a célula unitária $R$, podem ser escritas como:

$$
|R n\rangle=\frac{1}{2 \pi} \int_{-\pi}^{\pi} d k e^{-i k(R-x)}\left|u_{n k}\right\rangle
$$


A WCC $\bar{x}_{n}$ é definida como o valor esperado de $\langle 0 n|\hat{X}| 0 n\rangle$ do operador posição $\hat{X}$ no estado $|0 n\rangle$ correspondendo a uma das WF na célula com $R=0$. Equivalentemente temos:

$$
\bar{x}_{n}=\frac{i}{2 \pi} \int_{-\pi}^{\pi} d k\left\langle u_{n k}\left|\partial_{k}\right| u_{n k}\right\rangle .
$$

Levando em conta que temos $\sum_{\alpha} \bar{x}_{\alpha}^{S}=\frac{1}{2 \pi} \oint_{B Z} A^{S}$ com $S=I, I I$, onde a somatória em $\alpha$ representa estados ocupados e $A$ a conexão de Berry, obtemos:

$$
Z_{2}=\sum_{\alpha}\left[\bar{x}_{\alpha}^{I}(T / 2)-\bar{x}_{\alpha}^{I I}(T / 2)\right]-\sum_{\alpha}\left[\bar{x}_{\alpha}^{I}(0)-\bar{x}_{\alpha}^{I I}(0)\right]
$$

Assumindo que o calibre seja suave em $t \in[0, T / 2]$ é possível acompanhar a evolução dos WCCs durante meio cíclo, que também será suave. Um coisa importante a se notar é que a argumentação proposta aqui vale apenas quando:

- O calibre escolhido seja contínuo para cada tempo $t$, pois caso contrário isso poderia acarretar uma mudança de $Z_{2}$.

- Não ter quebra de TRS a qual é necessária para a degenerescência de pares de Kramer em $t=0$ e $t=T / 2$.

O calibre que possui as propriedades desejadas é chamado de $1 D$ maximally localized Wannier Function (MLWF). O critério [189] para a construção das MLWFs é que a escolha de calibre deve fornecer o mínimo possível da propagação quadrática $\Omega=\sum_{n}\left[\left\langle 0 n\left|\vec{r}^{2}\right| 0 n\right\rangle\right.$ $\left.-\langle 0 n|\vec{r}| 0 n\rangle^{2}\right]$. Em 1D os MLWF contruídos de acordo com este critério são autovalores do operador posição no espaço de bandas. Como este último comuta com $\Theta$ ainda existe degenerescência de Kramer em $t=0$ e $t=T / 2$. Para provar a continuidade do calibre, mostraremos aqui como fazê-lo em um mesh $k_{j+1}=k_{j}+\Delta k$ de uma construção de multibanda na zona de Brillouin. Em um dado ponto $t$ começando por $k=0$ contruímos matrizes de overlap $M_{m n}^{k_{j}, k_{j+1}}=\left\langle u_{m k_{j}} \mid u_{n k_{j+1}}\right\rangle$ de forma que sejam hermitianas. Repetindo o processo o estado $\left|\Psi_{n k}\right\rangle$ em $k=2 \pi$ estará relacionado com $k=0$ por uma rotação unitária cujo autovalor é $\lambda_{n}=e^{-i \bar{x}_{n}}$ que por sua vez proporciona o 1D MLWF. A continuidade é atingida olhando a evolução dos $\operatorname{WCC} \bar{x}_{n}(t)$ em função de $t$. Desta forma a equação (H.4) 
implica que monitorando continuamente a evolução dos dos pares do WCCs, podemos determinar de maneira simples o invariante $Z_{2}$.

\section{H.2 Procedimento matemático}

O que queremos resolver são os autovalores do operador $\hat{X}=\sum_{i \alpha} e^{-i \delta k_{x} \cdot \vec{R}_{i}}|\alpha i\rangle\langle\alpha i|$, onde $\delta k_{x}=\frac{2 \pi}{N_{x} a_{x}}$ com $N_{x}$ o número de células unitárias reais ao longo da direção x, $a_{x}$ é a constante de rede, $\alpha$ é o índice orbital e de spin, e $\vec{R}_{i}$ rotula a célula unitária. Notamos que a condição de contorno periódica é usado aqui. No limite de uma rede muito fina $\delta k_{x} \rightarrow 0$ e podemos definir o operador posição Hermitiano da seguinte forma:

$$
\hat{X}=\sum_{i \alpha} R_{i}|\alpha i\rangle\langle\alpha i|=i \frac{\partial}{\partial k_{x}}
$$

É mais conveniente para nós usar a condição de contorno periódica para efeitos de cálculo numérico. O operador $\hat{X}$ é um operador unitário cujo autovalores podem ser vistos como o centro de MLWF formado pelas bandas incluídas no operador $\hat{X}$. O conjunto de base localizada $\alpha$ é assumido completo, tais MLWFs são sempre bem definidas. Como apontado por Fu e Kane, o invariante topológico $Z_{2}$ pode ser determinado acompanhando a evolução dos centros de carga das funções de Wanniers para o sistema 1D com $k_{y}$ representando o tempo $t$ fixo no subespaço gerado por apenas as bandas ocupadas. O operador de projeção para o subespaço ocupado pode ser definido como:

$$
\hat{P}_{k_{y}}=\sum_{n \in o c c, k_{x}}\left|\Psi_{n, \vec{k}}\right\rangle\left\langle\Psi_{n, \vec{k}}\right|,
$$


onde occ representa o subespaço de estados ocupados. Assim ficamos com o seguinte operador posição:

$$
\begin{aligned}
\hat{X}_{p}\left(k_{y}\right) & =\hat{P}_{k_{y}} \hat{X} \hat{P}_{k_{y}}, \\
& =\sum_{n m \in o c c} \sum_{k_{x}, k_{x}^{\prime}, i \alpha} e^{-i \delta k_{x} \cdot \vec{R}_{i}}\left|\Psi_{n k_{x}, k_{y}}\right\rangle\left\langle\Psi_{n k_{x}, k_{y}}\right| \\
& \times|\alpha i\rangle\left\langle\alpha i \mid \Psi_{m k_{x}^{\prime}, k_{y}}\right\rangle\left\langle\Psi_{m k_{x}^{\prime}, k_{y}}\right| \\
& =\sum_{n m \in o c c} \sum_{k_{x}, k_{x}^{\prime}, i} \frac{1}{N_{c e l l}} e^{-i\left(k_{x}+\delta k_{x}-k_{x}^{\prime}\right) \cdot \vec{R}_{i}}\left|\Psi_{n k_{x}, k_{y}}\right\rangle\left\langle\Psi_{m k_{x}^{\prime}, k_{y}}\right| \\
& \times\left[\sum_{\alpha} g_{n \alpha}^{*}\left(k_{x}\right) g_{m \alpha}\left(k_{x}^{\prime}\right)\right] \\
& =\sum_{k_{x}, k_{x}^{\prime}} \delta\left(k_{x}+\delta k_{x}-k_{x}^{\prime}\right) \sum_{n m \in o c c}\left|\Psi_{n k_{x}, k_{y}}\right\rangle\left\langle\Psi_{m k_{x}^{\prime}, k_{y}}\right| \\
& \times\left[\sum_{\alpha} g_{n \alpha}^{*}\left(k_{x}\right) g_{m \alpha}\left(k_{x}^{\prime}\right)\right] .
\end{aligned}
$$

Em formato matricial ficamos com:

$$
\hat{X}_{p}\left(k_{y}\right)=\left[\begin{array}{llllll}
0 & F_{0,1} & 0 & 0 & \cdots & 0 \\
0 & 0 & F_{1,2} & 0 & \cdots & 0 \\
0 & 0 & 0 & F_{2,3} & \cdots & 0 \\
\vdots & \vdots & \vdots & \vdots & \vdots & \vdots \\
0 & 0 & 0 & 0 & 0 & F_{N_{x}-2, N_{x}-1} \\
F_{N_{x}-1,0} & 0 & 0 & 0 & \cdots & 0
\end{array}\right],
$$

onde temos:

$$
F_{i, i+1}^{n m}\left(k_{y}\right)=\sum_{\alpha} g_{n \alpha}^{*}\left(k_{x_{i}}, k_{y}\right) g_{m \alpha}\left(k_{x_{i+1}, k_{y}}\right) .
$$

Cada $F_{i, i+1}^{n m}\left(k_{y}\right)$ representa $2 N \times 2 N$ matrizes que geram $2 N$ autoestados ocupados. De qualquer forma não é necessário calcular explicitamente as funções de Wanniers, pois $F_{i, i+1}^{n m}\left(k_{y}\right)=\left\langle m k_{x_{i}} k_{y} \mid n k_{x_{i+1}} k_{y}\right\rangle$ e o problema de autovalores $\hat{X}_{p}\left(k_{y}\right)$ pode ser resolvido usando o método de matriz de transferência definindo um $D\left(k_{y}\right)=F_{0,1} F_{1,2} F_{2,3} \cdots F_{N_{x}-1,0}$. Esta última é uma matriz $2 N \times 2 N$ com $2 N$ autovalores dados por $\lambda_{n}=e^{-i \bar{x}_{n}}$. Como vimos o número $Z_{2}$ está relacionado com o número de enrolamento dos pares dos WCCs.

\section{H.3 Resultado e interpretação}

Na figura 52 temos a evolução dos WCCs para o caso do Siliceno. A troca de pares pode ser vista desenhando uma linha de referência (em vermelho) na figura (em qualquer lugar) na qual a evolução dos WCCs cruze esta linha um número ímpar de vezes.

Utilizamos esse procedimento para o Siliceno e concluímos que este é um isolante topológico, como esperado. Na figura 53 temos os casos de $\mathrm{Bi}_{2} \mathrm{Se}_{3}$ e $\mathrm{Sb}_{2} \mathrm{Se}_{3}$ em 3D. Nestes 


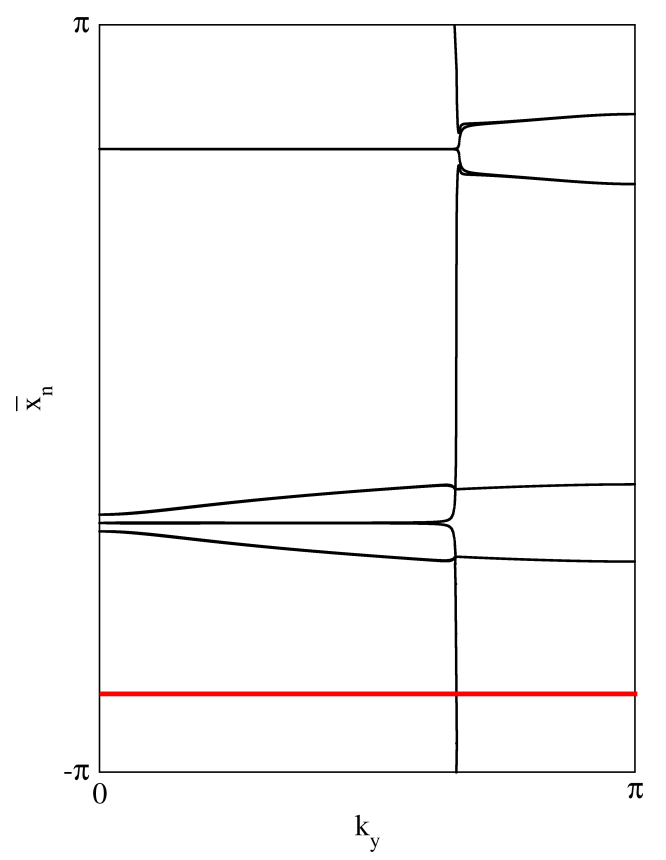

Figura 52: Evolução dos centros de carga de Wanniers WCCs para materiais 2D. Em vermelho temos a linha de referência. Se durante o meio ciclo entre $\Gamma$ e $M$ houver um número ímpar de cruzamentos entre os WCCs e a linha de referência temos um isolante topológico, caso contrário temos um isolante trivial.

sistemas, como vimos no corpo da tese, temos um invariante $Z_{2}$ atrelado a cada plano contendo 4 TRIMs distintos. Desta forma temos que olhar a evolução dos WCCs em dois planos distintos (aqui escolhemos $k_{z}=0$ e $k_{z}=\pi$ ). Um número ímpar e par de cruzamentos, entre a linha de referência e a evolução dos WCCs, na somatória de ambos os planos nos mostra que $\mathrm{Bi}_{2} \mathrm{Se}_{3}$ é um isolante topológico e $\mathrm{Sb}_{2} \mathrm{Se}_{3}$ é um isolante trivial. Esta ferramente será utilizada nos próximos trabalhos no qual a identificação da natureza topológica se torne necessária. 

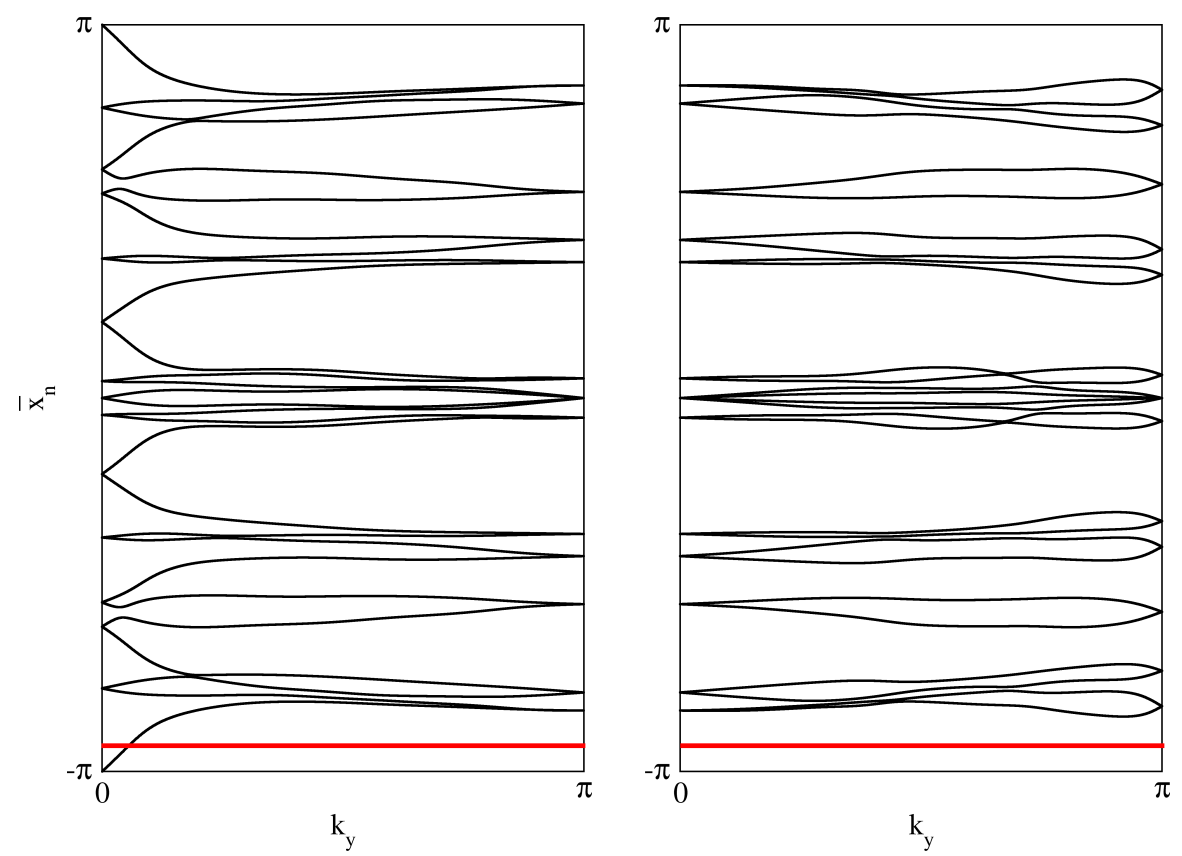

(a) WCCs para $\mathrm{Bi}_{2} \mathrm{Se}_{3}$ para o plano $k_{z}=0\left(k_{z}=\pi\right)$ à esquerda (direita).
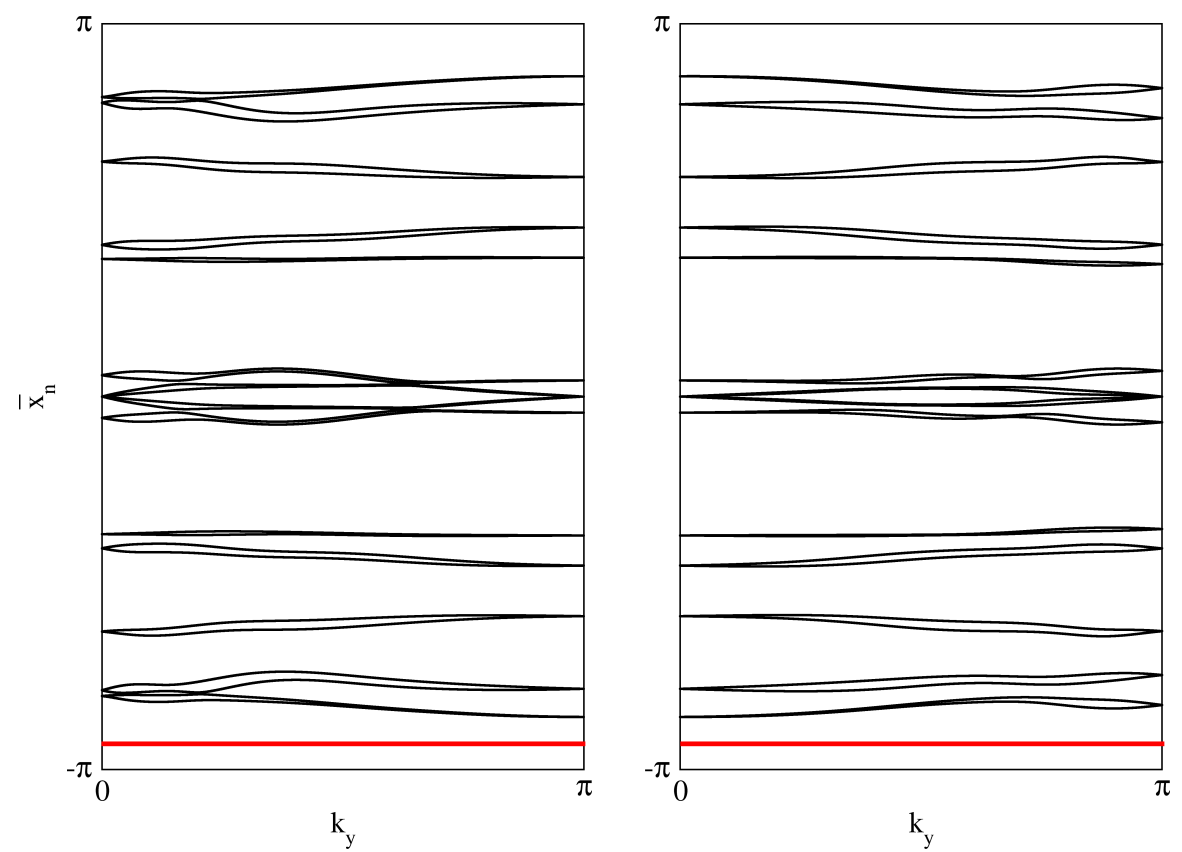

(b) WCCs para $S b_{2} S e_{3}$ para o plano $k_{z}=0\left(k_{z}=\pi\right)$ à esquerda (direita).

Figura 53: O mesmo que 52, para materiais 3D. Em vermelho temos a linha de referência. Se durante o meio ciclo entre $\Gamma$ e $M$ houver um número impar de cruzamentos entre os WCCs e a linha de referência para ambos os planos temos um isolante topológico, caso contrário temos um isolante trivial. 



\section{Referências}

[1] KLITZING, K. von; DORDA, G.; PEPPER, M. New method for high-accuracy determination of the fine-structure constant based on quantized hall resistance. Physical Review Letters, v. 45, p. 494-497, 1980.

[2] THOULESS, D. J. et al. Quantized hall conductance in a two-dimensional periodic potential. Physical Review Letters, v. 49, p. 405-408, 1982.

[3] XIAO, D. et al. Interface engineering of quantum hall effects in digital transition metal oxide heterostructures. Nature communications, v. 2, p. 596, 2011.

[4] KANE, C. L.; MELE, E. J. Quantum spin hall effect in graphene. Physical Review Letters, v. 95, p. 226801, 2005.

[5] KANE, C. L.; MELE, E. J. $\mathrm{Z}_{2}$ topological order and the quantum spin hall effect. Physical Review Letters, v. 95, p. 146802, 2005.

[6] HALDANE, F. D. M. Model for a quantum hall effect without landau levels: Condensed-matter realization of the parity anomaly. Physical Review Letters, v. 61, p. 2015-2018, 1988.

[7] WU, C.; BERNEVIG, B. A.; ZHANG, S.-C. Helical liquid and the edge of quantum spin hall systems. Physical Review Letters, v. 96, p. 106401, 2006.

[8] BERNEVIG, B. A.; HUGHES, T. L.; ZHANG, S.-C. Quantum spin hall effect and topological phase transition in HgTe quantum wells. Science (New York, N.Y.), v. 314, p. 1757-61, 2006. 
[9] KÖNIG, M. et al. Quantum spin hall insulator state in HgTe quantum wells. Science (New York, N.Y.), v. 318, p. 766-70, 2007.

[10] KÖNIG, M. et al. The quantum spin hall effect: Theory and experiment. Journal of the Physical Society of Japan, v. 77, p. 031007, 2008.

[11] KÖNIG, M. et al. Spatially resolved study of backscattering in the quantum spin hall state. Physical Review X, v. 3, p. 021003, 2013.

[12] FU, L.; KANE, C.; MELE, E. Topological insulators in three dimensions. Physical Review Letters, v. 98, p. 106803, 2007.

[13] MOORE, J.; BALENTS, L. Topological invariants of time-reversal-invariant band structures. Physical Review B, v. 75, p. 121306, 2007.

[14] ROY, R. $\mathrm{Z}_{2}$ classification of quantum spin hall systems: An approach using timereversal invariance. Physical Review B, v. 79, p. 195321, 2009.

[15] HSIEH, D. et al. A topological Dirac insulator in a quantum spin hall phase. Nature, v. 452, p. 970-4, 2008.

[16] LENOIR, B.; CASSART, M.; MICHENAUD, J.-P. Transport properties of Bi-rich Bi-Sb alloys. Journal of Physics and Chemistry of Solids, v. 57, p. 89-99, 1996.

[17] ZHANG, H. et al. Topological insulators in $\mathrm{Bi}_{2} \mathrm{Se}_{3}, \mathrm{Bi}_{2} \mathrm{Te}_{3}$ and $\mathrm{Sb}_{2} \mathrm{Te}_{3}$ with a single Dirac cone on the surface. Nature Physics, v. 5, p. 438-442, 2009.

[18] XIA, Y. et al. Observation of a large-gap topological-insulator class with a single Dirac cone on the surface. Nature Physics, v. 5, p. 398-402, 2009.

[19] KIM, S. et al. Surface scattering via bulk continuum states in the 3D topological insulator $\mathrm{Bi}_{2} \mathrm{Se}_{3}$. Physical Review Letters, v. 107, p. 056803, 2011.

[20] LIU, W. et al. Anisotropic interactions and strain-induced topological phase transition in $\mathrm{Sb}_{2} \mathrm{Se}_{3}$ and $\mathrm{Bi}_{2} \mathrm{Se}_{3}$. Physical Review B, v. 84, p. 245105, 2011. 
[21] VOGT, P. et al. Silicene: Compelling experimental evidence for graphenelike twodimensional silicon. Physical Review Letters, v. 108, p. 155501, 2012.

[22] XU, Y. et al. Large-gap quantum spin hall insulators in tin films. Physical Review Letters, v. 111, p. 136804, 2013.

[23] BIANCO, E. et al. Stability and exfoliation of germanane: a germanium graphane analogue. ACS nano, v. 7, p. 4414-21, 2013.

[24] SI, C. et al. Functionalized germanene as a prototype of large-gap two-dimensional topological insulators. Physical Review B, v. 89, p. 115429, 2014.

[25] YU, R. et al. Quantized anomalous Hall effect in magnetic topological insulators. Science (New York, N.Y.), v. 329, p. 61-64, 2010.

[26] PESIN, D.; MACDONALD, A. H. Spintronics and pseudospintronics in graphene and topological insulators. Nature materials, v. 11, p. 409-416, 2012.

[27] BORN, M.; OPPENHEIMER, R. Zur quantentheorie der molekeln. Ann. Phys. (Leipzig), v. 84 (20), p. 457, 1927.

[28] FEYNMAN, R. P. Forces in molecules. Physical Review, v. 56, p. 340-343, 1939.

[29] KRESSE, G.; FURTHMÜLLER, J. Efficiency of ab initio total energy calculations for metals and semiconductors using a plane wave basis set. Comput. Mat. Sci., v. 6, p. $15,1996$.

[30] KRESSE, G.; FURTHMÜLLER, J. Efficient iterative schemes for ab initio total energy calculations using a plane wave basis set. Physical Review B, v. 54, p. 11169, 1996.

[31] CAPELLE, K. A bird's-eye view of density-functional theory. Brazilian Journal of Physics, v. 36, p. 1318-1343, 2006.

[32] HOHENBERG, P.; KOHN, W. Inhomogeneous electron gas. Physical Review, v. 136, p. B864, 1964. 
[33] POPLE, J. A.; GILL, P. M.; JOHNSON, B. G. Kohn sham density functional theory within a finite basis set. Chemical Phys. Lett., v. 199 num 6, p. 557-560, 1992.

[34] KOHN, W.; SHAM, L. Self-consistent equations including exchange and correlation effects. Physical Review A, v. 140, p. 1133, 1965.

[35] HARTREE, D. R. The calculation of atomic structures. Rep. Prog. Phys., v. 11, p. 113, 1947.

[36] SLATER, J. C. The theory of complex spectra. Physical Review, v. 34, p. 1293, 1929.

[37] CONDOn, E. U. The theory of complex spectra. Physical Review, v. 36, p. 1121, 1930.

[38] LÖWDIN, P.-O. Correlation Problem in Many-Electron Quantum Mechanics I. Review of Different Approaches and Discussion of Some Current Ideas Advances in Chemical Physics. [S.1.]: John Wiley \& Sons, Inc., Hoboken, NJ, USA., 2007.

[39] FAZZIO, A.; CANUTO, S.; VIANNA, J. D. Teorica Quântica de Moléculas e Sólidos. [S.l.]: Livraria da Física São Paulo, 2004.

[40] SLATER, J. C.; KOSTER, G. F. Simplified LCAO method for the periodic potential problem. Physical Review, v. 94, p. 1498-1524, 1954.

[41] HERRING, C. A new method for calculating wave functions in crystals. Physical Review, v. 57, p. 1169-1177, 1940.

[42] SLATER, J. C. Wave functions in a periodic potential. Physical Review, v. 51, p. 846, 1937.

[43] KOHN, W.; ROSTOKER, N. Solution of the schrödinger equation in periodic lattices with an application to metallic lithium. Physical Review, v. 84, p. 1111-1120, 1954.

[44] KORRINGA, J. On the calculation of the energy of a bloch wave in a metal. Physica, v. 13 , p. $392-400,1947$. 
[45] CEPERLEY, D. M.; ALDER, B. J. Ground state of the electron gas by a stochastic method. Physical Review Letters, v. 45, p. 566-569, 1980.

[46] PERDEW, J. P.; BURKE, K.; ERNZENHOF, M. Generalized gradient approximation made simple. Physical Review Letters, v. 77, p. 3865, 1996.

[47] LEE, C.; YANG, W.; PARR, R. G. Development of the colle-salvetti correlationenergy formula into a functional of the electron density. Physical Review B, v. 37, p. $785,1988$.

[48] BECKE, D. Density-functional thermochemistry. iii. the role of exact exchange. Journal Chemical Phys, v. 98, p. 5648, 1993.

[49] PERDEW, J. P.; WANG, W. Accurate and simple density functional for the electronic exchange energy: Generalized gradient approximation. Physical Review B, v. 33, p. 88001, 1986.

[50] WIGNER, E. Group Theory and Its Application to the Quantum Mechanics of Atomic Spectra. [S.1.]: N.Y., Academic Press, 1959.

[51] BALDERESCHI, A. Mean-value point in the brillouin zone. Physical Review B, v. 7, p. $5212-5215,1973$.

[52] CHADI, D.; COHEN, M. Special points in the brillouin zone. Physical Review B, v. 8 , p. 5747,1973 .

[53] MONKHORST, H. J.; PACK, J. D. Special points for brillouin-zone integrations. Physical Review B, v. 13, p. 5188-5192, 1976.

[54] ARTACHO, E. et al. Linear-scaling ab initio calculations for large and complex systems. Phys. Stat. Sol. (b), v. 215, p. 809-817, 1999.

[55] OZAKI, T. et al. Open source package for Material eXplorer. 2008. Http://www.openmx-square.org/. 
[56] PHILliPS, J. C.; KLEINMAN, L. New method for calculating wave functions in crystals and molecules. Physical Review, v. 116, p. 287, 880, 1959.

[57] BACHELET, G. B.; HAMANN, D. R.; SCHLÜTER, M. Pseudopotentials that work: From H to Pu. Physical Review B, v. 26, p. 4199-4228, 1982.

[58] TROULLIER, N.; MARTINS, J. L. Efficient pseudopotentials for plane-wave calculations. Physical Review B, v. 43, p. 1993-2006, 1991.

[59] BLOCHL, P. Projector augmented-wave method. Physical Review B, v. 50, p. 17953, 1994.

[60] KRESSE, G.; JOUBERT, D. From ultrasoft pseudopotentials to the projector augmented-wave method. Physical Review B, v. 59, p. 1758, 1999.

[61] DIRAC, P. A. M. The quantum theory of the electron. Proc. R. Soc. London Ser. A, v. 117, p. $610,1928$.

[62] HASAN, M. Z.; KANE, C. Topological insulators. Reviews of Modern Physics, v. 82, p. $3045,2010$.

[63] ANDO, Y. Topological insulator materials. Phys. Soc. Japan, v. 82, p. 102001, 2013.

[64] BOHM A. MOSTAFAZADEH, H. K. Q. N. A.; ZWANZIGER, J. The Geometric Phase in Quantum Systems. [S.l.: s.n.], 2003.

[65] BERRY, M. Quantal phase factors accompanying adiabatic changes. Proceedings of the Royal Society of London, v. 392, p. 45-57, 1984.

[66] FU, L.; KANE, C. L. Time reversal polarization and a $Z_{2}$ adiabatic spin pump. Physical Review B, v. 74, p. 195312, 2006.

[67] FU, L.; KANE, C. Topological insulators with inversion symmetry. Physical Review $B$, v. 76 , p. $045302,2007$. 
[68] FUKUI, T.; HATSUGAI, Y. Quantum spin hall effect in three dimensional materials: Lattice computation of $\mathrm{Z}_{2}$ topological invariants and its application to $\mathrm{Bi}$ and $\mathrm{Sb}$. Journal of the Physical Society of Japan, v. 76, p. 053702, 2007.

[69] FUKUI, T.; FUJIWARA, T.; HATSUGAI, Y. Topological meaning of $\mathrm{Z}_{2}$ numbers in time reversal invariant systems. Journal of the Physical Society of Japan, v. 77, p. $123705,2008$.

[70] QI, X.-L.; HUGHES, T. L.; ZHANG, S.-C. Topological field theory of time-reversal invariant insulators. Physical Review B, v. 78, p. 195424, 2008.

[71] WANG, Z.; QI, X.-L.; ZHANG, S.-C. Equivalent topological invariants of topological insulators. New Journal of Physics, v. 12, p. 065007, 2010.

[72] RESTA, R. Theory of the electric polarization in crystals. Ferroelectrics, v. 136, p. $51-55,1992$.

[73] KING-SMITH, R. D.; VANDERBILT, D. Theory of polatization of crystalline solids. Physical Review B, v. 47, p. 1651, 1993.

[74] RESTA, R. Macroscopic polariation in crystalline dielectrics: the geometric phase approach. Reviews of Modern Physics, v. 66, p. 899, 1994.

[75] HOR, Y. et al. p-type $\mathrm{Bi}_{2} \mathrm{Se}_{3}$ for topological insulator and low-temperature thermoelectric applications. Physical Review B, v. 79, p. 195208, 2009.

[76] HSIEH, D. et al. A tunable topological insulator in the spin helical Dirac transport regime. Nature, v. 460, p. 1101-5, 2009.

[77] PARK, K. et al. Robustness of topologically protected surface states in layering of $\mathrm{Bi}_{2} \mathrm{Te}_{3}$ thin films. Physical Review Letters, v. 105, p. 186801, 2010.

[78] CHEN, Y. L. et al. Experimental realization of a three-dimensional topological insulator, $\mathrm{Bi}_{2} \mathrm{Te}_{3}$. Science (New York, N.Y.), v. 325, p. 178-81, 2009. 
[79] HSIEH, D. et al. Observation of time-reversal-protected single-Dirac-cone topological-insulator states in $\mathrm{Bi}_{2} \mathrm{Te}_{3}$ and $\mathrm{Sb}_{2} \mathrm{Te}_{3}$. Physical Review Letters, v. 103, p. 146401, 2009.

[80] NISHIDE, A. et al. Direct mapping of the spin-filtered surface bands of a threedimensional quantum spin Hall insulator. Physical Review B, v. 81, p. 041309, 2010.

[81] TEO, J. C. Y.; FU, L.; KANE, C. L. Surface states and topological invariants in three-dimensional topological insulators: Application to $\mathrm{Bi}_{1-x} \mathrm{Sb}_{x}$. Physical Review B, v. 78, p. $045246,2008$.

[82] ZHANG, H.-J. et al. Electronic structures and surface states of the topological insulator $\mathrm{Bi}_{1-x} \mathrm{Sb}_{x}$. Physical Review B, v. 80, p. 085307, 2009.

[83] PARK, S. R. et al. Quasiparticle scattering and the protected nature of the topological states in a parent topological insulator $\mathrm{Bi}_{2} \mathrm{Se}_{3}$. Physical Review B, v. 81, p. 041405, 2010.

[84] ZHANG, W. et al. First-principles studies of the three-dimensional strong topological insulators $\mathrm{Bi}_{2} \mathrm{Te}_{3}, \mathrm{Bi}_{2} \mathrm{Se}_{3}$ and $\mathrm{Sb}_{2} \mathrm{Te}_{3}$. New Journal of Physics, v. 12, p. 065013, 2010.

[85] HE, K. et al. Crossover of the three-dimensional topological insulator $\mathrm{Bi}_{2} \mathrm{Se}_{3}$ to the two-dimensional limit. Nature Physics, v. 6, p. 584-588, 2010.

[86] KURODA, K. et al. Experimental realization of a three-dimensional topological insulator phase in ternary chalcogenide TlBiSe 2 . Physical Review Letters, v. 105, p. 146801, 2010.

[87] DAI, X.-Q. et al. Robust surface state of intrinsic topological insulator $\mathrm{Bi}_{2} \mathrm{Te}_{2} \mathrm{Se}_{\text {thin }}$ films: a first-principles study. Journal of physics. Condensed matter, v. 24, p. 035502, 2012.

[88] YOUNG, S. M. et al. Theoretical investigation of the evolution of the topological phase of $\mathrm{Bi}_{2} \mathrm{Se}_{3}$ under mechanical strain. Physical Review B, v. 84, p. 035502, 2011. 
[89] JARIWALA, B.; SHAH, D. Stacking fault in $\mathrm{Bi}_{2} \mathrm{Te}_{3}$ and $\mathrm{Sb}_{2} \mathrm{Te}_{3}$ single crystals. Journal of Crystal Growth, v. 318, p. 1179-1183, 2011.

[90] MEDLIN, D. L. et al. Structure of the (0001) basal twin boundary in $\mathrm{Bi}_{2} \mathrm{Te}_{3}$. Journal of Applied Physics, v. 108, p. 043517, 2010.

[91] LIND, H.; LIDIN, S. A general structure model for Bi-Se phases using a superspace formalism. Solid State Sciences, v. 5, p. 47-57, 2003.

[92] LIND, H.; LIDIN, S.; HÄUSSERMANN, U. Structure and bonding properties of $\left(\mathrm{Bi}_{2} \mathrm{Se}_{3}\right)_{m}\left(\mathrm{Bi}_{2}\right)_{n}$ stacks by first-principles density functional theory. Physical Review B, v. 72 , p. $184101,2005$.

[93] CHOU, M. Y.; COHEN, M. L.; LOUIE, S. G. Theoretical study of stacking faults in silicon. Physical Review B, v. 32, p. 7979, 1985.

[94] YAZYEV, O.; MOORE, J.; LOUIE, S. Spin polarization and transport of surface states in the topological insulators $\mathrm{Bi}_{2} \mathrm{Se}_{3}$ and $\mathrm{Bi}_{2} \mathrm{Te}_{3}$ from first principles. Physical Review Letters, v. 105, p. 266806, 2010.

[95] SEIXAS, L. et al. Topological states ruled by stacking faults in $\mathrm{Bi}_{2} \mathrm{Se}_{3}$ and $\mathrm{Bi}_{2} \mathrm{Te}_{3}$. Journal of Applied Physics, v. 113, p. 023705, 2013.

[96] QI, X.-L.; ZHANG, S.-C. Topological insulators and superconductors. Reviews of Modern Physics, v. 83, p. 1057-1110, 2011.

[97] WANG, Y.-L. et al. Structural defects and electronic properties of the Cu-doped topological insulator $\mathrm{Bi}_{2} \mathrm{Se}_{3}$. Physical Review B, v. 84, p. 075335, 2011.

[98] HOR, Y. S. et al. Development of ferromagnetism in the doped topological insulator $\mathrm{Bi}_{2-x} \mathrm{Mn}_{x} \mathrm{Te}_{3}$. Physical Review B, v. 81, p. 195203, 2010.

[99] HOR, Y. S. et al. Superconductivity in $\mathrm{Cu}_{x} \mathrm{Bi}_{2} \mathrm{Se}_{3}$ and its implications for pairing in the undoped topological insulator. Physical Review Letters, v. 104, p. 057001, 2010. 
[100] ZHANG, D. et al. Interplay between ferromagnetism, surface states, and quantum corrections in a magnetically doped topological insulator. Physical Review B, v. 86, p. 205127, 2012.

[101] SCHOLZ, M. et al. Tolerance of topological surface states towards magnetic moments: Fe on $\mathrm{Bi}_{2} \mathrm{Se}_{3}$. Physical Review Letters, v. 108, p. 256810, 2012.

[102] WRAY, L. A. et al. A topological insulator surface under strong coulomb, magnetic and disorder perturbations. Nature Physics, v. 7, p. 32-37, 2011.

[103] CHEN, Y. L. et al. Massive Dirac fermion on the surface of a magnetically doped topological insulator. Science (New York, N.Y.), v. 329, p. 659-62, 2010.

[104] XU, S.-Y. et al. Hedgehog spin texture and berry's phase tuning in a magnetic topological insulator. Nature Physics, v. 8, p. 616-622, 2012.

[105] VALLA, T. et al. Photoemission spectroscopy of magnetic and nonmagnetic impurities on the surface of the $\mathrm{Bi}_{2} \mathrm{Se}_{3}$ topological insulator. Physical Review Letters, v. 108, p. 117601, 2012 .

[106] HAAZEN, P. P. J. et al. Ferromagnetism in thin-film Cr-doped topological insulator $\mathrm{Bi}_{2} \mathrm{Se}_{3}$. Applied Physics Letters, v. 100, p. 082404, 2012.

[107] CHOI, Y. H. et al. Transport and magnetic properties of $\mathrm{Cr}-$, Fe-, Cu-doped topological insulators. Journal of Applied Physics, v. 109, p. 07E312, 2011.

[108] LIU, Q. et al. Magnetic impurities on the surface of a topological insulator. Physical Review Letters, v. 102, p. 156603, 2009.

[109] LI, Z. et al. Strong single-ion anisotropy and anisotropic interactions of magnetic adatoms induced by topological surface states. Physical Review B, v. 85, p. 054426, 2012.

[110] JIN, H.; SONG, J.-H.; FREEMAN, A. Dirac cone engineering in $\mathrm{Bi}_{2} \mathrm{Se}_{3}$ thin films. Physical Review B, v. 83, p. 125319, 2011. 
[111] HENK, J. et al. Complex spin texture in the pure and Mn-doped topological insulator $\mathrm{Bi}_{2} \mathrm{Te}_{3}$. Physical Review Letters, v. 108, p. 206801, 2012.

[112] JIN, K.-H.; JHI, S.-H. Effect of atomic impurities on the helical surface states of the topological insulator $\mathrm{Bi}_{2} \mathrm{Te}_{3}$. Journal of physics. Condensed matter : an Institute of Physics journal, v. 24, p. 175001, 2012.

[113] SCHMIDT, T. M.; MIWA, R. H.; FAZZIO, A. Spin texture and magnetic anisotropy of Co impurities in $\mathrm{Bi}_{2} \mathrm{Se}_{3}$ topological insulators. Physical Review B, v. 84, p. 245418, 2011.

[114] HONOLKA, J. et al. In-plane magnetic anisotropy of fe atoms on $\mathrm{Bi}_{2} \mathrm{Se}_{3}(111)$. Physical Review Letters, v. 108, p. 256811, 2012.

[115] HENK, J. et al. Topological character and magnetism of the Dirac state in Mndoped $\mathrm{Bi}_{2} \mathrm{Te}_{3}$. Physical Review Letters, v. 109, p. 076801, 2012.

[116] ZHANG, S.; NORTHRUP, J. Chemical potential dependence of defect formation energies in GaAs: Application to Ga self-diffusion. Physical Review Letters, v. 67, p. 2339-2342, 1991.

[117] PHILIPSEN, P.; BAERENDS, E. Cohesive energy of 3d transition metals: Density functional theory atomic and bulk calculations. Physical review. B, Condensed matter, v. 54, p. 5326-5333, 1996.

[118] KITTEL, C. Introduction to Solid State Physics. [S.l.]: Wiley, 2004.

[119] SIDORKO, V. R.; GONCHARUK, L. V.; ANTONENKO, R. V. Thermodynamic properties of bismuth sesquiselenide and sesquitelluride and their solid solutions. Powder Metallurgy and Metal Ceramics, v. 47, p. 3-4, 2008.

[120] TERSOFF, J.; HAMANN, D. R. Theory of the scanning tunneling microscope. Physical Review B, v. 31, p. 805-813, 1985. 
[121] URAZHDIN, S. et al. Scanning tunneling microscopy of defect states in the semiconductor $\mathrm{Bi}_{2} \mathrm{Se}_{3}$. Physical Review B, v. 66, p. 161306, 2002.

[122] YAN, B.; ZHANG, D.; FELSER, C. Topological surface states of $\mathrm{Bi}_{2} \mathrm{Se}_{3}$ coexisting with Se vacancies. physica status solidi (RRL) - Rapid Research Letters, v. 7, p. 148150, 2013.

[123] BYCHKOV, Y.; RASHBA, E. Properties of a 2D electron gas with lifted spectral degeneracy. JETP letters, v. 39, p. 78, 1984.

[124] DIL, J. H. Spin and angle resolved photoemission on non-magnetic low-dimensional systems. Journal of physics. Condensed matter : an Institute of Physics journal, v. 21, p. 403001, 2009.

[125] ZHANG, J.-M. et al. Tailoring magnetic doping in the topological insulator $\mathrm{Bi}_{2} \mathrm{Se}_{3}$. Physical Review Letters, v. 109, p. 266405, 2012.

[126] WEST, D. et al. Identification of magnetic dopants on the surfaces of topological insulators: Experiment and theory for fe on $\mathrm{Bi}_{2} \mathrm{Te}_{3}(111)$. Physical Review B, v. 85, p. 081305, 2012.

[127] YE, M. et al. Quasiparticle interference on the surface of $\mathrm{Bi}_{2} \mathrm{Se}_{3}$ induced by cobalt adatom in the absence of ferromagnetic ordering. Physical Review B, v. 85, p. 205317, 2012.

[128] ABDALLA, L. B. et al. Topological insulator $\mathrm{Bi}_{2} \mathrm{Se}_{3}(111)$ surface doped with transition metals: An ab initio investigation. Physical Review B, v. 88, p. 045312, 2013.

[129] CHECKELSKY, J. et al. Quantum interference in macroscopic crystals of nonmetallic $\mathrm{Bi}_{2} \mathrm{Se}_{3}$. Physical Review Letters, v. 103, p. 246601, 2009.

[130] ANALYTIS, J. G. et al. Bulk fermi surface coexistence with Dirac surface state in $\mathrm{Bi}_{2} \mathrm{Se}_{3}$ : A comparison of photoemission and shubnikov-de haas measurements. Physical Review B, v. 81, p. 205407, 2010. 
[131] ETO, K. et al. Angular-dependent oscillations of the magnetoresistance in $\mathrm{Bi}_{2} \mathrm{Se}_{3}$ due to the three-dimensional bulk fermi surface. Physical Review B, v. 81, p. 195309, 2010 .

[132] BUTCH, N. P. et al. Strong surface scattering in ultrahigh-mobility $\mathrm{Bi}_{2} \mathrm{Se}_{3}$ topological insulator crystals. Physical Review B, v. 81, p. 241301, 2010.

[133] SMITH, M. J.; KNIGHT, R. J.; SPENCER, C. W. Properties of $\mathrm{Bi}_{2} \mathrm{Te}_{3}-\mathrm{Sb}_{2} \mathrm{Te}_{3}$ alloys. Journal of Applied Physics, v. 33, p. 2186, 1962.

[134] REN, Z. et al. Optimizing $\mathrm{Bi}_{2-x} \mathrm{Sb}_{x} \mathrm{Te}_{3-y} \mathrm{Se}_{y}$ solid solutions to approach the intrinsic topological insulator regime. Physical Review B, v. 84, p. 165311, 2011.

[135] TASKIN, a. a. et al. Observation of Dirac holes and electrons in a topological insulator. Physical Review Letters, v. 107, p. 016801, 2011.

[136] TANG, J. et al. Electrical detection of spin-polarized surface states conduction in $\left(\mathrm{Bi}_{0.53} \mathrm{Sb}_{0.47}\right)_{2} \mathrm{Te}_{3}$ topological insulator. Nano letters, 2014.

[137] NIU, C. et al. Realization of tunable Dirac cone and insulating bulk states in topological insulators (bi(1-x)sb(x))(2)te(3). Scientific reports, v. 2, p. 976, 2012.

[138] XU, S. et al. Discovery of several large families of topological insulator classes with backscattering-suppressed spin-polarized single-Dirac-cone on the surface. arXiv preprint, 2010.

[139] REN, Z. et al. Large bulk resistivity and surface quantum oscillations in the topological insulator $\mathrm{Bi}_{2} \mathrm{Te}_{2}$ Se. Physical Review B, v. 82, p. 241306, 2010.

[140] XIONG, J. et al. Quantum oscillations in a topological insulator $\mathrm{Bi}_{2} \mathrm{Te}_{2} \mathrm{Se}$ with large bulk resistivity $(6 \omega \mathrm{cm})$. Physica E: Low-dimensional Systems and Nanostructures, v. 44, p. 917-920, 2012.

[141] ZUNGER, A. et al. Special quasirandom structures. Physical Review Letters, v. 65, p. $353-356,1990$. 
[142] LI, W. et al. Pressure-induced topological quantum phase transition in $\mathrm{Sb}_{2} \mathrm{Se}_{3}$. Physical Review B, v. 89, p. 035101, 2014.

[143] ANDERSON, T.; KRAUSE, H. Refinement of the $\mathrm{Sb}_{2} \mathrm{Te}_{3}$ and $\mathrm{Sb}_{2} \mathrm{Te}_{2} \mathrm{Se}$ structures and their relationship to nonstoichiometric $\mathrm{Sb}_{2} \mathrm{Te}_{3-y} \mathrm{Se}_{y}$ compounds. Crystallographica Section B: Structural, v. 30, p. 1307, 1974.

[144] EZAWA, M.; TANAKA, Y.; NAGAOSA, N. Topological phase transition without gap closing. Scientific reports, v. 3, p. 2790, 2013.

[145] LIU, J.; VANDERBILT, D. Topological phase transitions in $\left(\mathrm{Bi}_{1-x} \operatorname{In}_{x}\right)_{2} \mathrm{Se}_{3}$ and $\left(\mathrm{Bi}_{1-x} \mathrm{Sb}_{x}\right)_{2} \mathrm{Se}_{3}$. Physical Review B, v. 88, p. 224202, 2013.

[146] MIN, H. et al. Intrinsic and rashba spin-orbit interactions in graphene sheets. Physical Review B, v. 74, p. 165310, 2006.

[147] YAO, Y. et al. Spin-orbit gap of graphene: First-principles calculations. Physical Review B, v. 75, p. 041401, 2007.

[148] LIU, C. C.; FENG, W. X.; YAO, Y. G. Quantum spin hall effect in silicene and two-dimensional germanium. Physical Review Letters, v. 107, p. 076802, 2011.

[149] PADILHA, J. E. et al. Quantum spin hall effect in a disordered hexagonal $\mathrm{Si}_{x} \mathrm{Ge}_{1-x}$ alloy. Physical Review B, v. 88, p. 201106, 2013.

[150] CHUANG, F.-C. et al. Prediction of large-gap two-dimensional topological insulators consisting of bilayers of group iii elements with Bi. Nano Letters, v. 14, p. 2505$2508,2014$.

[151] MA, Y. et al. Halogenated two-dimensional germanium: candidate materials for being of quantum spin hall state. Journal of Materials Chemistry, v. 22, p. 1258712591, 2012 
[152] LIU, C.-C. et al. Low-energy effective hamiltonian for giant-gap quantum spin hall insulators in honeycomb X-hydride/halide $(\mathrm{X}=\mathrm{N} / \mathrm{Bi})$ monolayers. Physical Review B, v. 90, p. 085432, 2014.

[153] DÁVILA, M. E. et al. Germanene: a novel two-dimensional germanium allotrope akin to graphene and silicene. New Journal of Physics, v. 16, p. 095002, 2014.

[154] SEIXAS, L.; PADILHA, J. E.; FAZZIO, A. Quantum spin hall effect on germanene nanorod embedded in completely hydrogenated germanene. Physical Review B, v. 89, p. 195403, 2014.

[155] MATTHES, L.; BECHSTEDT, F. Influence of edge and field effects on topological states of germanene nanoribbons from self-consistent calculations. Physical Review B, v. 90, p. 165431, 2014.

[156] WANG, X.-Q.; LI, H.-D.; WANG, J.-T. Induced ferromagnetism in one-side semi hydrogenated silicene and germanene. Physical Chemistry Chemical Physics, v. 14, p. 3031-3036, 2012.

[157] OZAKI, T. Variationally optimized atomic orbitals for large-scale electronic structures. Physical Review B, v. 67, p. 155108, 2003.

[158] THEURICH, G.; HILL, N. Self-consistent treatment of spin-orbit coupling in solids using relativistic fully separable ab initio pseudopotentials. Physical Review B, v. 64, p. 073106, 2001.

[159] RIBAS, M. A. et al. Patterning nanoroads and quantum dots on fluorinated graphene. Nano Research, v. 4, p. 143-152, 2011.

[160] SINGH, A. K.; YAKOBSON, B. Electronics and magnetism of patterned graphene nanoroads. Nano Letters, v. 9, p. 1540-1543, 2009.

[161] ALMEIDA, J. M. et al. Electronics and magnetism of patterned graphene nanoroads. Nanotechnology, v. 24, p. 495201, 2013. 
[162] LIAO, L.; PENG, H.; LIU, Z. Chemistry makes graphene beyond graphene. Journal of the American Chemical Society, v. 136, p. 12194-12200, 2014.

[163] KASHTIBAN, R. J. et al. Atomically resolved imaging of highly ordered alternating fluorinated graphene. Nature Communications, v. 5, p. 4902, 2014.

[164] KARLICKÝ, F. et al. Halogenated graphemes: rapidly growing family of graphene derivatives. ACS Nano, v. 7, p. 6434-6464, 2013.

[165] BRUNO, G. et al. Photothermally controlled structural switching in fluorinated polyene-graphene hybrids. Physical Chemistry Chemical Physics, v. 16, p. 13948, 2014.

[166] ROBINSON, J. T. et al. Properties of fluorinated graphene films. Nano Letters, v. 10, p. 3001-3005, 2010.

[167] SIMEK, P. et al. Towards graphene iodide: Iodination of graphite oxide. Nanoscale, DOI10.1039, p. C4NR05219F, 2014.

[168] YAO, Z. et al. Catalyst-free synthesis of iodine-doped graphene via a facile thermal annealing process and its use for electrocatalytic oxygen reduction in an alkaline medium. Chemical Communications, v. 48, p. 1027-1029, 2012.

[169] BINNIG, G. et al. Surface studies by scanning tunneling microscopy. Scanning Tunneling Microscopy, v. 49, p. 57-61, 1982.

[170] BINNIG, G. Tunneling through a controllable vacuum gap. Applied Physics Letters, v. 40, p. $178,1982$.

[171] BINNIG, G.; ROHRER, H. Scanning tunneling microscopy. Surface Science Letters, v. 126, p. $236-244,1983$.

[172] BINNIG, G.; ROHRER, H. 7 x 7 reconstruction on $\mathrm{Si}(111)$ resolved in real space. Physical Review Letters, v. 50, p. 120, 1983.

[173] BINNIG, G. et al. (111) facets as the origin of reconstructed Au (110) surfaces. Surface Science Letters, v. 131, p. 379-384, 1983. 
[174] CURIE, J.; CURIE, P. Sur l'électricité polaire dans les cristaux hémièdres à faces inclinées. Comptes rendus de l'Académie des Sciences, v. 91, p. 383-6, 1880.

[175] CURIE, J.; CURIE, P. Développement, par pression, de l'électricité polaire dans les cristaux hémièdres à faces inclinées. Comptes rendus de l'Académie des Sciences, v. 91, p. $294-5,1880$.

[176] CURIE, J.; CURIE, P. Contractions et dilatations produites par des tensions dans les cristaux hémièdres à faces inclinées. Comptes rendus de l'Académie des Sciences, v. 93, p. 1137-1140, 1880.

[177] BARDEEN, J. Tunneling from a many-particle point of view. Physical Review Letters, v. 6, p. 57-59, 1961.

[178] NØRDHEIM, L. The electron theory of metals. Ann. Phys. (Leipzig), v. 9, p. 607, 1931.

[179] SOVEN, P. Coherent-potential model of substitutional disordered alloys. Physical Review Letters, v. 156, p. 809-813, 1967.

[180] WEI, S.-H. et al. Electronics properties of random alloys: Special quasirandom structures. Physical Review B, v. 42, p. 9622-9650, 1990.

[181] VENEZUELA, P. et al. Ab initio determination of the atomistic structure of $\mathrm{Si}_{x} \mathrm{Ge}_{1-x}$ alloy. Physical Review B, v. 64, p. 193202, 2001.

[182] DAMASCELLI, A. Probing the electronic structure of complex systems by ARPES. Physica Scripta, v. 109, p. 61-74, 2004.

[183] HUFNER, S. Photoelectron Spectroscopy. [S.l.]: Springer, 1995.

[184] GAY, T. J.; DUNNING, F. B. Mott electron polarimetry. Review of Scientific Instruments, v. 63, p. 1635-1651, 1991.

[185] RESTA, R.; VANDERBILT, D. Theory of polarization: A modern approach. Physics of Ferroelectrics, p. 31-68, 2007. 
[186] ANDO, T.; NAKANISHI, T. Impurity scattering in carbon nanotubes - absence of back scattering -. Journal of Physical Society of Japan, v. 67, p. 1704, 1988.

[187] SOLUYANOV, A.; VANDERBILT, D. Computing topological invariants without inversion symmetry. Physical Review B, v. 83, p. 235401, 2011.

[188] YU, R. et al. Equivalent expression of $z_{2}$ topological invariant for band insulators using the non-abelian berry connection. Physical Review B, v. 84, p. 075119, 2011.

[189] MARZARI, N.; VANDERBILT, D. Maximally localized generalized wannier functions for composite energy bands. Physical Review B, v. 56, p. 12847-12865, 1997. 